\title{
Multilingual Practices and Identity Negotiation among Multilingual Heritage Language Learners in New Zealand
}

\author{
By
}

Mohammed Yousef Nofal

\begin{abstract}
A thesis
submitted to the Victoria University of Wellington in fulfilment of the requirements for the degree of Doctor of Philosophy in Linguistics
\end{abstract}

Victoria University of Wellington 2020 
To

Hanadi

who will remember the time and place if not the people,

Sara, Sireen, Mahamoud, and Salma

who filled my life with joy, euphoria and love 


\begin{abstract}
While heritage languages (HLs) have been receiving much research attention, there is still a scarcity of studies conducted on local HL communities. However, researchers in New Zealand have been actively engaged with various community languages for over four decades, providing rich insights into the dynamics of language maintenance and language shift within these communities. Although New Zealand sociolinguistic scholarship has covered a wide range of languages and ethnicities, there is no known study on the Indian Hindi community, whose HL is the fourth most spoken language in the country (Statistics New Zealand, 2013). Additionally, previous research has traditionally examined the functional aspects of language use and language attitudes in determining whether language can be preserved, viewing HL communities often as homogeneously formed. In contrast, current trends in the field of sociolinguistics aim to examine the connections between individuals and their languages (i.e. identity), taking multilingualism as a norm and focusing on dynamism in intraspeaker and interspeaker language use. This thesis addresses these issues by exploring how the realities that heritage language learners (HLLs) live connect to identity negotiation and development in social interaction. In particular, this thesis focuses on a group of learners of Hindi as a heritage language in New Zealand - a group that is under-explored.
\end{abstract}

Grasping the relationship between the HLLs' experiences and how they develop and negotiate heritage-related identities necessitates a micro-level analysis of language use by casting an eye on language practices in the language maintenance school and the home, for they constitute two key spaces of exposure to the HLs and cultures. Moreover, examining how HLLs draw upon indexicality to conceptualise their languages provides rich insights into their identity negotiation and development.

The primary data for the analyses is mobilised in three dimensions adopting an ethnographic approach. The first dimension includes limited-participant observations for one school term, making a total of 20 hours of observation out of which 12 hours were recorded. The observations look at language practices in a multi-site Hindi School (HS) where families of Indian descent from various linguistic, ethnic, cultural and national backgrounds come together forming a constellation of communities of practice (Lave \& Wenger, 1991; Wenger, 1998b) to stay connected with their Indian heritage. I also conducted semi-structured interviews with eight parents and stakeholders in the HS to enrich the analysis and check my interpretations of the observed and recorded practices. 
The second dimension embraces recordings of home interaction within three families with the aim of exploring language practices in the home. A total of eight hours of recorded data were collected in different conversational encounters (e.g. in the car, at the dining table and playtime). The families participating in this research have unique characteristics in terms of their heterogeneous configuration. The first family exemplifies a transnational adoptive family which is a unique family structure that has not been researched in New Zealand. The other two families reflect multicultural New Zealand Indian families where the parents do not speak the same HL. Finally, the data in the third dimension comes from the learners through linguistic reflection drawings (Krumm \& Jenkins, 2001; Seals, 2017b). Twenty HLLs participated in the drawing activity which aims at examining how they process meaning-making through the use of language-colour association and views the linguistic repertoire as embodied (Bucholtz \& Hall, 2016; Krumm \& Jenkins, 2001).

By employing the concept of communities of practice during in-depth discourse analysis, the HS data suggests that the shared practices within the school contribute to the construction of the learners' multilingual and national/cultural identities, emphasising the Indian identity as an overarching one (i.e. Indianness), rather than privileging other regional, national or religious identities.

Additionally, the analysis of the home data suggests that no matter how committed community members are, the HL is not always actively used at home. Rather, the three families in this study take part in a wide range of language practices that index their Indian identities. They introduce aspects of the Indian culture, which is mostly indexed via music, food and cultural lexical items in their discourse (Friesen, 2008; Shah, 2013). While HL literacy skills (e.g. numeracy and the reading of literary texts) were elicited, English linguistic features that are often associated with Indian English were used to construct Indian identity. However, at times multiple memberships became problematic because it contradicted other socially constructed identities, depending on the membership that is activated in the interaction settings. The analysis offers insights into the complexities of discursive identity negotiation within the home and the intricate relationship between identity negotiation and multiple memberships.

Finally, the analysis of the HLLs' linguistic reflection drawings through an indexical lens (Ochs, 1993) reveals that the participants use their languages as direct indices to display forms of capital (Bourdieu, 1986), which in turn are discursively used to index national and cultural identities. Likewise, some participants used their multilingual identities as a resource to negotiate national and/or cultural identities. 
Overall, this thesis sheds light on the complexities of identity negotiation and development in heterogeneous communities where community members have multiple heritage languages. As this research is the first to present non-traditional language school and family configurations in the New Zealand context, it will hopefully enrich the understanding of the dynamics of heritage language education and identity negotiation in such superdiverse settings. 


\section{Acknowledgements}

$$
\text { يا رب لك الحمد كما ينبني لجلال وجهك وعظيم سلطانك، ويوافي سابغ نعمك وجزيل إحسانك }
$$

First and foremost, all praise is due to God's noble face, His immense authority, and His glorious blessings as much as it suits His glory and great power, and as much as it meets His countless blessings and abundant beneficence.

It is a chance of a lifetime to acknowledge the help and support I received throughout this journey. I must confess that if I were to mention those whose souls, voices and actions shaped this work, many would be left unnamed.

To my beloved family, thank you for encouragement, support and endless love all the way of my pursuits. I am especially grateful to the reason of my being, my parents, who have burnt the candle at both ends, raising me and instilling in me humanistic and knowledge-seeking to reach to this stage at the expense of their wellbeing, though happily. To them, I would like to say "your efforts were in no vain", so thank you.

To my wife, Hanadi, and my children, Sara, Sireen, Mahmoud and Salma: It would be very remiss of me not to apologise for not being physically with you over the past three years. I apologise for every moment you felt sad for not having me with you. I am truly indebted to you, Hanadi, for doing your best to raise the four lovely, though hyperactive, kids on your own in my absence.

My brothers Ahamed, Othman, Hasan, Ali and Ibrahim, and my sisters Jazia, Wafa', Thana' and $S a f a$ ': there are no words to express my gratitude adequately for your love and care. I cherish the matchless love my children and I received over the years.

I was fortunate enough to have not one but two wonderful supervisors, who believed in me when I was not at my best, pushed me to the best of my abilities and opened my mind to new things. Their invaluable insights, comments and suggestions profoundly shaped this thesis. My sincere gratitude goes to primary supervisor A/Prof. Corinne Seals and my secondary supervisor Prof. Meredith Marra for their continuous support, patience, motivation, and immense knowledge. Thanks for reading and commenting on the countless number of drafts at all stages of my study.

I would also like to thank the thesis committee: Dr Margaret Gleeson, Dr Arianna BerardiWiltshire, and Dr Kendall King, for their insightful suggestions and constructive feedback. 
To my friends at VUW, Mohammed Al-Zeer, Mohammed Ali Hashmi, Yasir Altaf, Abdelmalik Hasanain and Baligh Al-Hilali for being on my side over the years of study. Their advice, support and discussions enriched my understanding from different perspectives. I shall not forget to thank my friend and mentor Prof. Bader Dweik for teaching me how to lead when yesterday's enthusiasm becomes today's yawn; to my friends Saleh Al-Abwini, Tareq Refa'i, Ahmad Mansour, Abd Makawi, Nasir Qandil and Ehab Abu Ijmail for emailing and calling, giving me the feeling of being home. My appreciation goes also to Hanadi Qawar for her support and the rich discussions we had over the topic.

I would like to thank Victoria University of Wellington for supporting this research through the three-year Victoria Doctoral Scholarship and the Victoria Submission Scholarship. Special thanks are extended to the School of Linguistics and Applied Language Studies family for the supporting and inspiring environment, particularly, Janet Attril, Bernie Hambleton, Matthew Vink, Alastair Baldwin, Carlos Espinoza, and Patrick Coelho and many others who tirelessly work for our benefit.

Finally but most importantly my appreciation goes to my participants who accepted and welcomed me in their community. I also thank the Hindi School, namely Sunita Narayan, Vijeshni Rattan, for their enthusiasm, encouragement and efforts to make this research successful. 


\section{Table of contents}

Chapter One: Introduction .................................................................................................................. 15

A personal anecdote: ................................................................................................................................. 15

1.1 Rationale and aims of the thesis............................................................................................... 16

1.2 Indian communities in New Zealand ..................................................................................... 18

1.3 Historical linguistic background ............................................................................................. 20

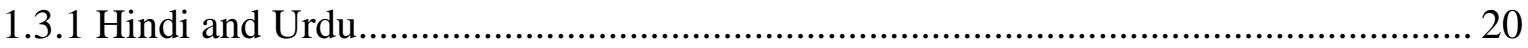

1.3.2 Ausbau process as language policy and planning in India........................................ 22

1.3.3 The development of Fiji Hindi........................................................................... 24

1.4 Hindi in society ................................................................................................................................. 26

1.5 Setting the scene.............................................................................................................................. 28

1.6 Thesis overview....................................................................................................................................... 30

Chapter Two: Review of literature ................................................................................................... 32

2.1 Introduction .............................................................................................................................................. 32

2.2 LMLS: Bird's eye view of the field ........................................................................................... 33

2.3 Multilingualism and LMLS.......................................................................................................... 36

2.4 Heritage language education ................................................................................................. 37

2.5 Communities of practice and investment................................................................................. 40

2.6 Shifting focus: Language and identity .................................................................................. 45

2.6.1 Identity and interaction .................................................................................. 45

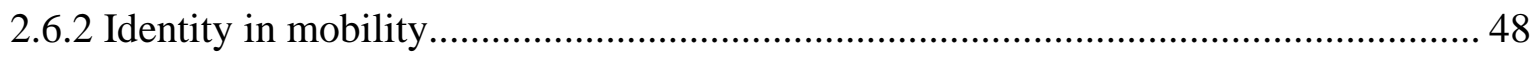

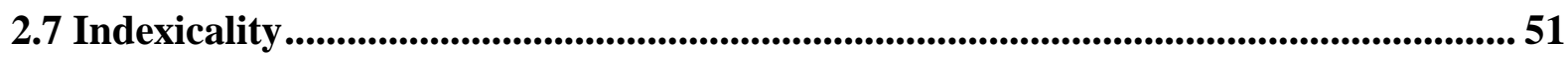

2.8 Gaps and research questions ...................................................................................................... 54

Chapter Three: Methodology ............................................................................................................... 57

3.1 Introduction .............................................................................................................................. 57

3.2. Why an ethnographic approach?............................................................................................ 58 
3.3.1 Gaining access to the community

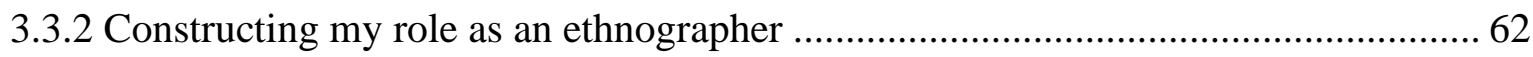

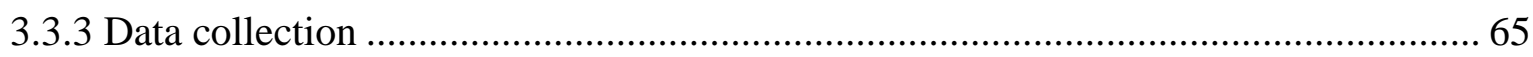

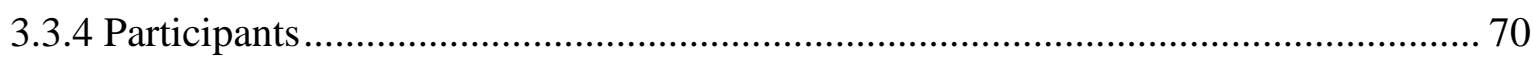

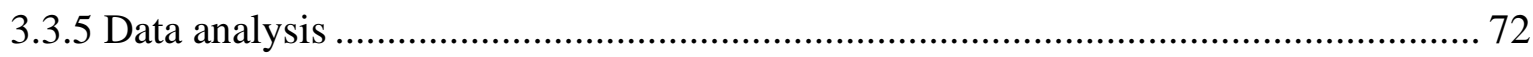

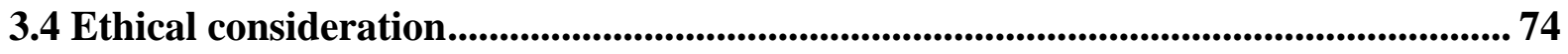

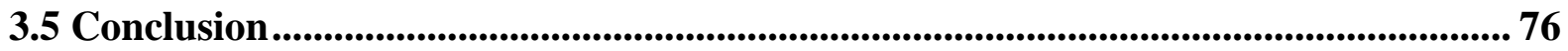

Chapter Four: Language practices in the HL school........................................................ 77

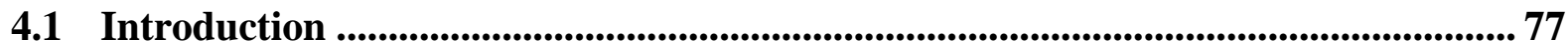

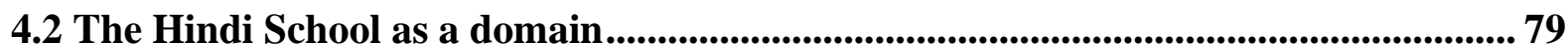

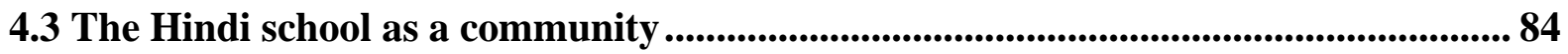

4.4 Practices in the Hindi School................................................................................ 88

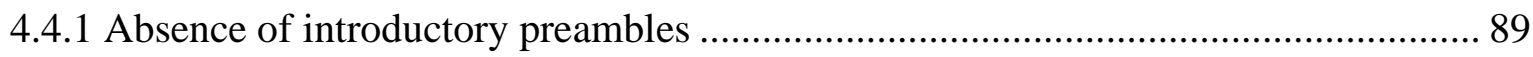

4.4.2 The ability to assess the appropriate practice....................................................... 91

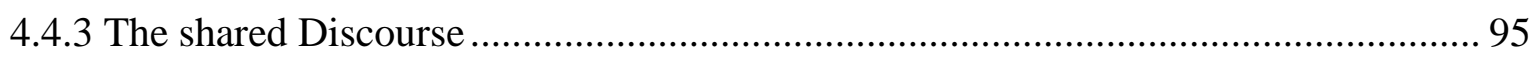

4.4.3.1 Terms of address (all sites): Set-up, repertoire policing ................................... 99

4.4.3.2 Namaste: Creating boundaries and repertoire policing ................................ 100

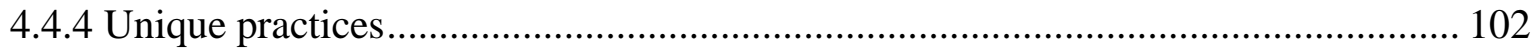

4.4.4.1 Language play at site B: Humour, translanguaging and styling.................... 102

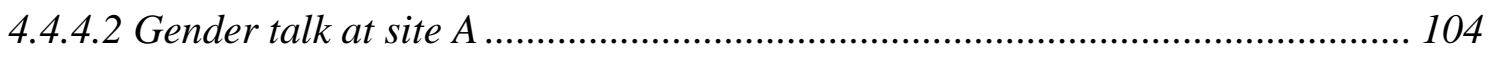

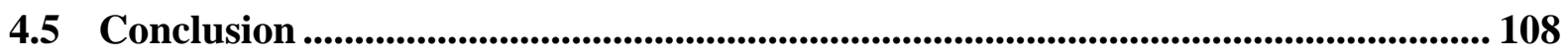

Chapter Five: Language, capital and identity construction: Indexicalities................... 111

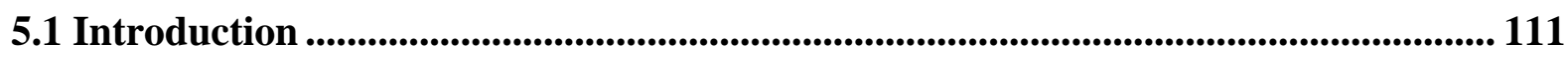

5.2 Linguistic reflection drawings .................................................................................. 111

5.3 Language as capital: Indexing identity ................................................................................... 113

5.3.1 Hindi as social capital, HL linguistic capital: Indexing national identity ............... 114

5.3.2 Hindi as cultural capital: Indexing cultural identity............................................ 119

5.3.3 HL as cultural capital: Indexing cultural and national identities ........................... 124 
5.4.1 Multilingualism and multiculturalism as a capital: Indexing the Kiwi identity

5.4.2 Learner linguistic identity: Indexing the Indian cultural identities

5.5 Conclusion

Chapter Six: Language practices in the home.

6.1 Introduction

6.2 Muromaha's family

6.2.1 Meta-discussion around national identity

6.2.1.1 Positioning leading to othering

6.2.1.2 Rejection of positioning (being othered) .................................................... 143

6.2.1.3 Complex and contradicting membership ..................................................... 145

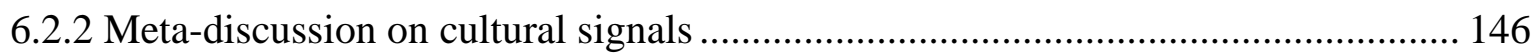

6.2.3 Linguistic meta-discussion (positioning child as an expert) ................................. 150

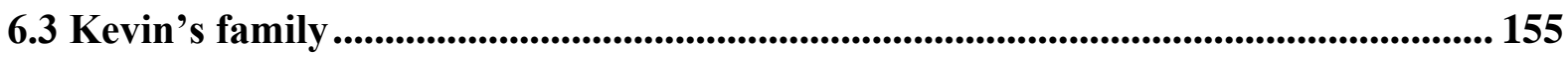

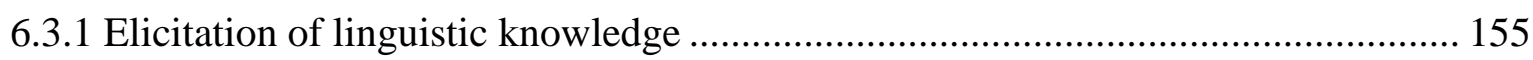

6.4 Kaya ji's family ................................................................................................................................. 158

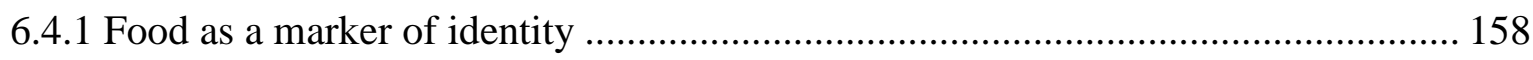

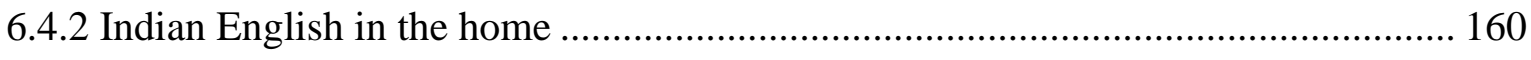

6.5 Conclusion................................................................................................................................................... 162

Chapter Seven: Discussion ............................................................................................. 164

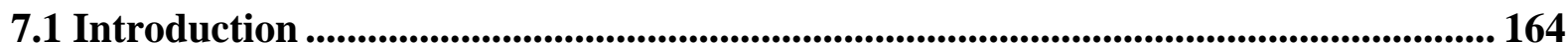

7.2 The Indian national/cultural identity in the HS ................................................... 164

7.3 Multilingual identity development ................................................................................................ 167

7.4 Linguistic and national/cultural identities in multiple memberships...................... 169

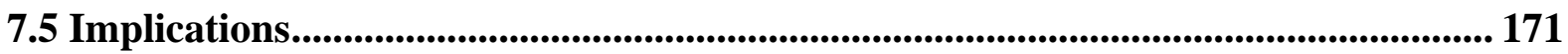

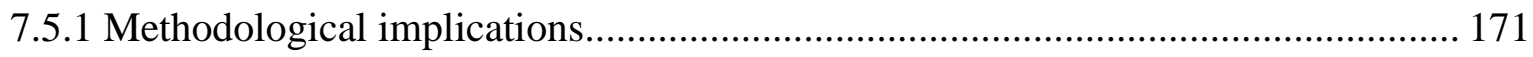

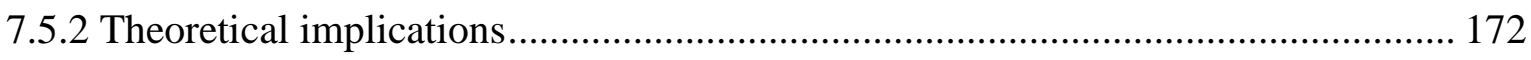

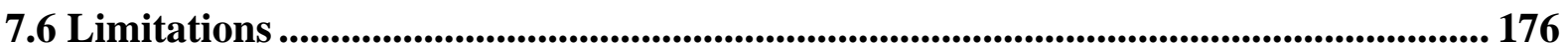




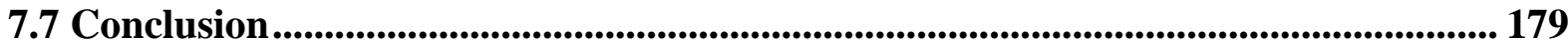

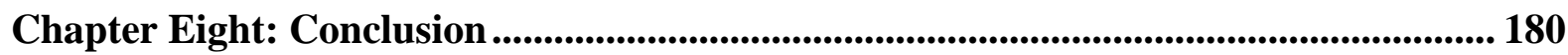

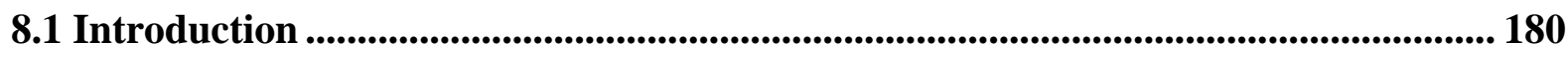

8.2 Summary of research findings .................................................................................................. 181

8.4 Future directions for research........................................................................... 184

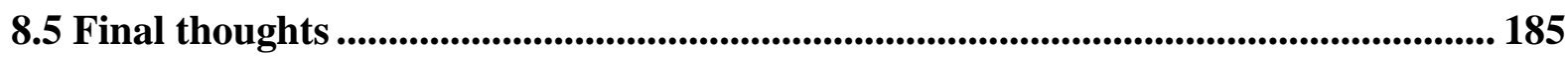

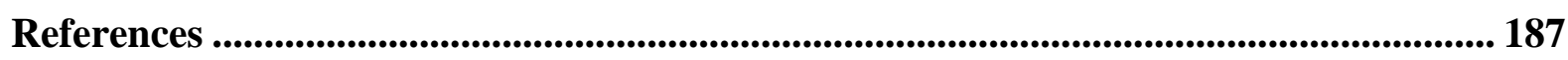

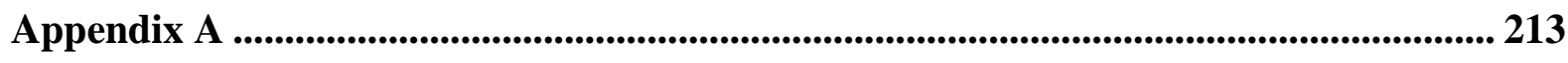

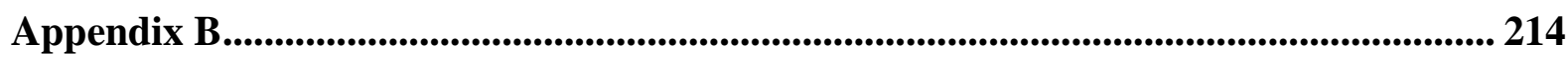

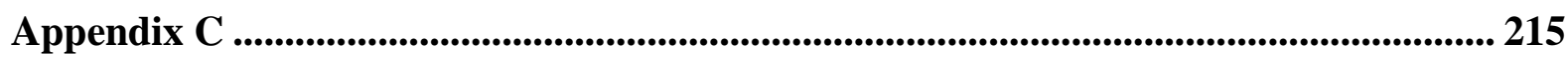

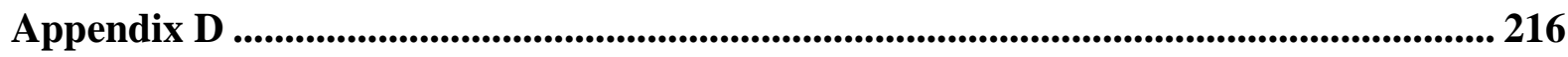

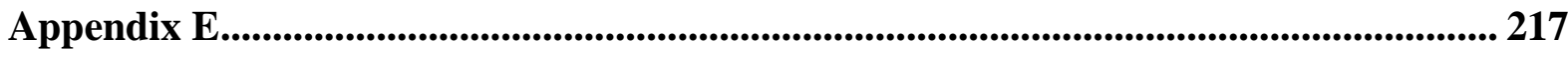

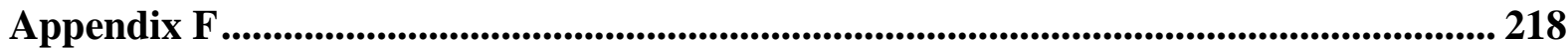




\section{Table of figures and images}

Figure 1.1 The Hindi community in New Zealand ........................... 20

Figure 1.2 Hindustani and the development of Hindi and Urdu ................. 22

Figure 2.1 Model of a communicative event ............................ 47

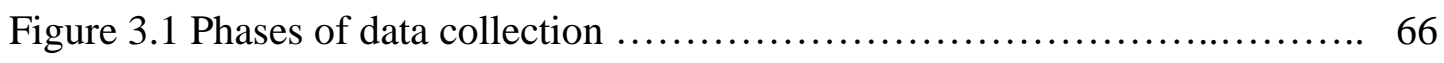

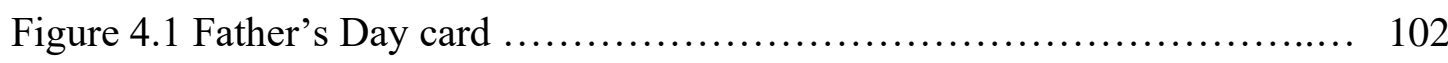

Image 5.1 Tattu's linguistic reflection drawing .......................... 116

Image 5.2 Ann's linguistic reflection drawing ............................ 121

Image 5.3 Lucy's linguistic reflection drawing ........................... 125

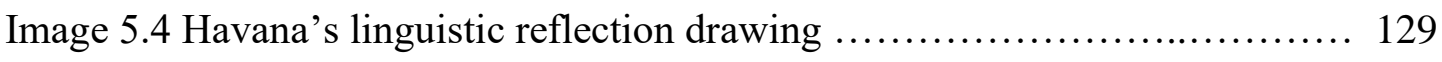

Image 5.5 Lara's linguistic reflection drawing ........................... 133 


\section{Abbreviations}

CofP Community of Practice

FH Fiji Hindi

FLP Family Language Policy

HL Heritage Language

HLE Heritage Language Education

HLL Heritage Language Learner

HS Hindi School

LE Linguistic Ethnography

LM Language Maintenance

LMLS Language Maintenance and Language Shift 


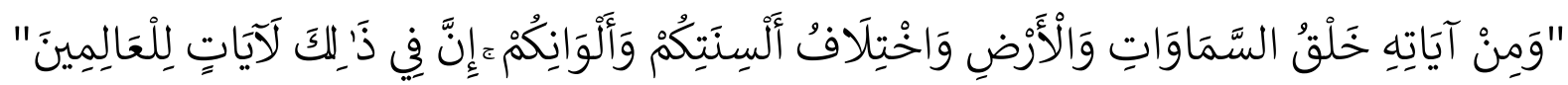
And among His signs is the creation of the heavens and the earth, and the difference of your languages and colours. Verily, in that are indeed signs for those of sound knowledge.

(Ar Rum: 22) 


\section{Chapter One: Introduction}

This thesis delves into the relationship between the HLLs' experiences and how they develop and negotiate heritage-related identities. This necessitates a micro-level analysis of their language use, which is achieved by casting an eye on language practices in the language maintenance school and the home. These constitute two key spaces of exposure to the HLs and cultures. In this chapter, I provide a holistic picture of the research background and settings. I first describe the impetus for my interest in heritage languages, followed by outlining the rationale and goal of the thesis. I then provide an account of the presence of the Indian communities in New Zealand along with a historical linguistic account of (Indian) Hindi, Fiji Hindi (FH) and Urdu to show why Hindi matters for Indians. The chapter concludes by elaborating on the research settings and offering an overview of the thesis structure.

\section{A personal anecdote:}

In Aden-Yemen, where my interest in linguistics began, I spent four years as an international undergraduate student from Jordan (2000-2004). While Yemen is 'politically' seen as a monolingual country, with Arabic as the one and only official language by constitution, my personal experience in that country nurtured my knowledge regarding linguistic minorities and led me to probe into the issue of languages in contact.

One day while a friend of mine, who was of Indian descent, and I were passing by a neighbourhood in Crater-Aden, we came across a group of local children (aged between 7 to 12) playing football with non-Arabic comments and calls. It was the first time I had heard their language (which I could not identify). When I asked about the language they spoke, my friend recounted the historical narrative of who they were and how they became Yemenis. My friend explained that the children were of Indian descent and that their families had moved to Yemen when Aden was annexed to the British colonies in the 1860s. My friend then added that the boys were using their Indian heritage languages, including Hindi and Gujarati, which the Indian communities in that neighbourhood used at home and amongst one another.

This experience motivated my interest in studying the factors that helped them to maintain these language Indian communities in an Arabic "monolingual" country. This research became my MA thesis in (2011). That project sparked further interest leading me to think of whether linguistic minorities, especially Indian groups, keep their heritage in multilingual contexts, e.g. New Zealand. Thus, my interest has turned to the Hindi communities in New Zealand, whose 
heritage language (HL) is the fourth most used (with no institutional support). I wanted to explore how they "do" their Indian identities discursively.

\subsection{Rationale and aims of the thesis}

In addition to my personal interest, the impetus for this research derives from the literature on multilingualism and linguistic diversity which has recently shifted its focus from migration to mobility. This shift has resulted from a more heterogeneous understanding of globalisation.

Language maintenance and language shift research has always viewed the home as the most important domain for maintaining heritage languages (Fishman, 1991a; Spolsky, 2004). This has been supported in a wide range of studies, but the dynamicity and heterogeneity of HL communities and their related configurations (e.g. family and school) suggest that what 'counts' in the process of language maintenance is more complex. For example, the emergence of Family Language Policy (FLP) as a distinct field within language maintenance research heightens the critical role of family and the home in raising an HL speaker (Schwartz \& Verschik, 2013), but also challenges traditional views of family configurations. The concept of a family is more diverse than the stereotypical view in language maintenance and language shift (LMLS), including adoptive and single-parent families inter alia. Similarly, the emergence of Heritage Language Education (HLE) illustrates the pivotal role of HL schools in HL maintenance, especially HL literacy (Oriyama, 2010, 2016). HLE has promoted the recognition of HLs in mainstream school programmes (Seals \& Peyton, 2016) and created innovative classrooms, language policies and practices within HL communities and beyond. While acknowledging the complementary relationship between the home and HL schools, investigating actual language practices within the context of the home remains uncommon (Fogle, 2012; King, 2017; Seals \& Shah, 2017). This indicates a fruitful gap for exploring multilingual practices of HL learners/speakers both within the family and at HL schools to capture their lived experiences and, in particular, how these experiences connect to identity. This thesis aims to address nontraditional family configurations as found amongst the families in the HL school under investigation, namely a weekend school characterised by superdiversity (including a transnational adoptive family as well as multilingual multicultural families). This study aims to bring about greater understanding of the depth and breadth of LMLS as the focus changes from the lens of the more traditional linguistic socialisation into ‘cultural socialisation' (Shin, 2013). 
This study is also necessary in the New Zealand context. HLE and LMLS research in New Zealand has flourished since the 1980s. Researchers have addressed community languages (the term most frequently used in New Zealand literature at the time) from various perspectives (Holmes, Roberts, Verivaki, \& 'Aipolo, 1993; Revis, 2015; Seals \& Olsen-Reeder, 2017; Walker, 2011). However, while the focus has been on what is typically understood as homogenous categories such as ethnicity and the speech community, there is a paucity of studies which interrogate more complex and heterogeneous configurations. The superdiverse characteristics of the Indian community in this study (which enjoys various linguistic, ethnic, national, and cultural backgrounds) create a need for exploring other possibilities when researching such a group. This need becomes more pressing when we consider the fact that the Hindi communities of New Zealand have received little scholarly attention. Thus, this thesis aims to contribute to LMLS research in the New Zealand context as well as internationally by drawing a picture of how the discourse of belonging in superdiverse settings contributes to identity.

Methodologically, this thesis responds to calls for using innovative research methods in LMLS in particular and sociolinguistics at large. For example, this thesis brings together an analysis of the conversattions with young participants, discussing their drawings to verbally express their lived experiences (Melo-Pfeifer, 2017; Seals, 2017b), and the sociolinguistic concept of indexicality (Ochs, 1993) to signal social meaning. Likewise, this study incorporates recordings of naturally occurring interactions to capture a glimpse of the practices within both the family and the HL school, resonating with current practices in the field of sociolinguistics in which micro levels of analysis are favoured.

Last but not least, this thesis has the goal of being beneficial to the community by providing documented evidence for Hindi community members to use to argue for the immersion of Hindi in state-run schools in line with new language education policies in the country. Further, while the focus of this thesis is not pedagogical, HL schools and curriculum designers can benefit from the language practice described in the study to develop materials that have the potential to enhance HLLs' investment in language learning as well as their connection to their HL identities.

The purpose of this thesis is to examine the interplay between the sociocultural dynamics extant in HLLs' lives and HLLs' identity negotiation and development. That is, how the societal, cultural and linguistic realities of school and home inform how HLLs connect to their linguistic and national/cultural identities in social interaction. Bearing in mind the complexity and 
dynamism of the community, I chose to conduct a qualitative study, incorporating ethnographic and discourse analysis approaches because these afford a holistic picture of the dynamic configuration of the school communities and access to deep insights into the interactional complexities of the community. To operationalise the objective of this thesis, I worked with a community language Hindi School and the communities attached to it. I here emphasize the plural form 'communities' rather than the singular due to the wide variety of linguistic, cultural and national backgrounds of the HS members as outlined in the remainder of this thesis. My participants were primarily teachers, students and the families affiliated with the HS. Prior to giving an account of the context of this research, I shall provide a socio-historical background of the Indian communities in New Zealand, followed by historical linguistic background of Hindi, Urdu and Fiji Hindi.

\subsection{Indian communities in New Zealand}

The history of Indian ${ }^{1}$ immigration to New Zealand shows that there were various periods of immigration, with earlier arrivals of Indians in 1810 (Leckie, 1995). The Indian migration from the Gujarat and Punjab provinces started on a small scale in the 1890s for economic, political and demographic reasons (Tiwari, 1980). In the region of Gujarat, the economic decline was considered a major migration factor and, in the region of Punjab, emigration was triggered by overpopulation and underemployment, alongside the decline of industries and a shortage of water (Tiwari, 1980). Most of the emigrants were traders, farmers, artisans or small businessmen. The number of Indian immigrants increased steadily until the 1920s when the Immigration Restriction Amendment Act of 1921 restricted the 'Asian' influx into New Zealand. The main source of Indian migration after 1921 was through the arrival of the children, wives and fiancées of earlier migrants (Friesen, 2008). Furthermore, immigration declined due to the Imperial Conference held in 1918 that confirmed that each community in the British Empire had the right to control the composition of its own population by imposing restrictions on immigration from any of the other communities (Atkinson, 2016). Compounding this was an ideological restriction whereby Indians were a target of the racist campaign of the White New Zealand League in 1926. This league comprised Pukekohe businessmen who opposed Asian immigration, especially those from China and India, because

\footnotetext{
1 The term Indian in this thesis refers to people native or inhabitant of India, or a person of Indian descent.
} 
they were perceived as a threat to the racial integrity and economic prosperity of European New Zealanders (Johnson \& Moloughney, 2006; Wanhalla, 2013).

After World War II, although restrictions on Asian immigration continued, small numbers of Indians arrived under special permissions category, including family reunification conditions and as students. The arrival of migrants from Fiji also increased the proportion of Indians (Taher, 1970). Friesen (2008, p. 47) reported that "at the 1986 census there were 14,172 people who identified themselves as Indian and this made up less than one half of 1 per cent $(0.4$ per cent) of New Zealand's population". The Indian influx into New Zealand accelerated between 1987 and 2000 due to political changes in New Zealand and Fiji. New Zealand Immigration Policy changed in the late 1980s giving a visa waiver to Indo-Fijians after the 1987 coup in Fiji (Friesen, 2008). During this period 16,000 Indo-Fijians migrated to New Zealand after the military coup (Narayan \& Smyth, 2003). The number of Indo-Fijian immigrants reached around 6000 in 2006 (Pio, 2008). Additionally, results of the 2013 census show that the Indian population in New Zealand totalled 62,187 people in 2001, 104,583 in 2006 and reached 155,178 in 2013 (Statistics New Zealand, 2013).

New Zealand has a variety of Indian communities, distinguished by place of origin (e.g. Asian Indians and Indo-Fijians), religion (e.g. Hindu, Muslims, Christians and Sikhs) and language. Members of these communities speak a variety of languages, e.g. Hindi $(66,312)$ speakers, Panjabi (19,752), Gujarati (17,505), Malayalam (4,365) and Fiji Hindi (1,674) (Statistics New Zealand, 2013). In the Wellington region in 2013, as compared to Indo-Fijians who counted 10,929 people, the Asian Indian population was 13,581 people (or $9.5 \%$ of the total population of New Zealand and 3.7\% of the population of the Wellington region) (Statistics New Zealand, 2013). In recent years, Indian migration to New Zealand has gradually increased again, further raising the number of speakers of Indian languages. Among these languages, Hindi has recently replaced French as the fourth most common language in New Zealand after English, Te Reo Māori and Samoan (Statistics New Zealand, 2013). Due to historical and societal reasons as noted in the following section, the Hindi community in New Zealand can be thought of as living in a multi-layer sphere as shown in Figure 1.1. The Hindi community lies in the smallest domain with Hindi as a unifying linguistic factor among its speakers whether as a first, second or heritage language. Going to the middle domain, the Indian culture and nationalism construct the unifying force where different languages and ethnicities can be found - this will be discussed in Chapter Four. The third and biggest domain forms the New Zealand wider society where Indians and other groups share identities as New Zealanders. 


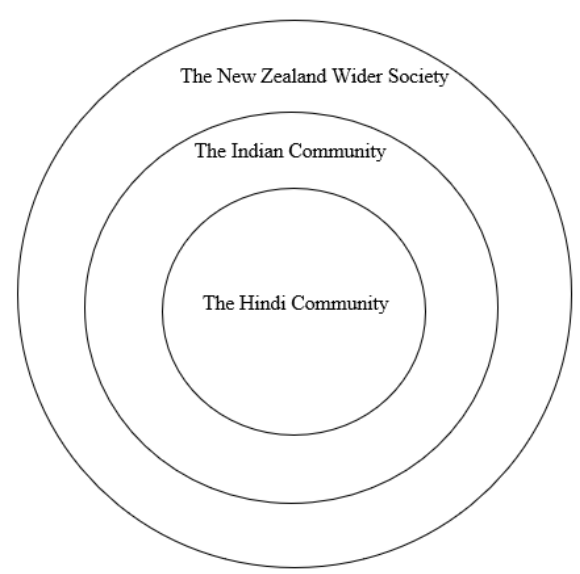

Figure 1.1 The Hindi community in New Zealand

One of the distinctive features is that "most Asian Indians are multilingual. They have partial competence in each of the languages in their verbal repertoire" (Sridhar, 2002, p. 276). That is, members of Indian communities are expected to be knowledgeable about different languages without necessarily being aware of the linguistic systems of the languages in an individual's repertoire, (as explored in Chapter Four). The Hindi speaking population is 66,312 (or 1.56\%) people in New Zealand and 5,262 people (1.12\%) in Wellington ${ }^{2}$. This also equals $33 \%$ of the Asian Indian population in Wellington. As a particular group, 1,674 people report that they speak Fiji Hindi as their first language in 2013 (Statistics New Zealand, 2013), and another 5000 are speakers of Urdu which has a high degree of intelligibility with Hindi. In the following section, by overviewing how Hindi and its mutually intelligible 'languages' Fiji Hindi and Urdu evolved, I aim to provide a background of why Hindi "matters" for Indians.

\subsection{Historical linguistic background}

\subsubsection{Hindi and Urdu}

In this section, I briefly discuss the sociolinguistic situation of India, especially the historical and linguistic background of Hindi and Urdu. I discuss these two language labels from two perspectives. While the first is a historical viewpoint, encompassing socio-political aspects, the second focuses on a linguistic viewpoint, as ausbau languages that evolved due to deliberate language planning.

Hindi evolved under historical and socio-political influences to the extent that its own name 'Hindi' is a Persian word that was used to address Indian people and languages (Verma (1933)

\footnotetext{
${ }^{2}$ According to the 2013 Census, the Wellington region is the third-most populous in New Zealand, with $11.1 \%$ (369,606 people) of the population. It follows Auckland (with 33.4 percent of the nation's population, or $1,415,550$ people) and Canterbury (12.7 percent, or 539,436 people).
} 
cited in Kachru, 2009). Prior to its most recent name, Hindi was known by a variety of names, e.g. Hindavi, Hindui, Hindwi, Rexta (mixed language), Dakhini and Hindustani (Dua, 2006; Kachru, 2009; Shukla, 2006). After the Persian invasion of India and the establishment of the Islamic rule in the North, the emergent language of the 'market place' and 'army camps' had been called 'Khari boli'. Khari boli was used for communication among Afghans, Persians, Turks and the local people (Kachru, 2009, p. 400). Kahri boli is considered the mother of Hindustani, which is a central Indo-Aryan spoken language (Dua, 2006; Shameem, 1995; Shukla, 2006). Hindustani, aka 'Hindi-Urdu' (Siegel, 1987), refers to a colloquial form whose written form is now viewed as two distinct languages, i.e. Hindi and Urdu. This neutral common spoken language was adopted at the beginning of the twentieth century as a lingua franca to serve as a symbol of national identity in India (Kachru, 2009) instead of the religiousidentity related to Hindi and Urdu. On the one hand, Hindi is a modern Indo-Aryan language, spoken by almost $45 \%$ of the population in India (Shukla, 2006). It is also used in various countries, e.g. Nepal, Gayana, Singapore and South Africa (Lewis, Simons, \& Fennig, 2017), ranking as the fifth language in the world after Chinese, Spanish, English and Arabic (Lewis et al., 2017) . It is the first and second language of more than 300 million speakers (Kachru, 2009), and serves as a lingua franca in India and in parts of the Indian diaspora (Mufwene, 2010). It is also one of the supercentral languages that serve a purpose of international communication (Ammon, 2010; De Swaan, 2010).

One the other hand, Urdu is the native language of about 23 million people in India and 8 million in Pakistan (Kachru, 2009). The word 'urdu' derived from Turkish 'ordu' which means 'camp', and the term was not used to denote the language till the $18^{\text {th }}$ century (Dua, 2006; Kachru, 2009). Since Urdu was used at Muslim courts and in literature during the Islamic rule, it was socially constructed as a language associated with Islam. In contrast, the variety used by general people, Hindi, borrowed the Sanskrit conventions for literary works (Kachru, 2009). Later on, Hindi was represented as a language associated with Hinduism (Shameem, 1995). Seemingly, politics has played a major role in dividing the Hindustani language into two separate languages. In this regard, Christopher (1994, p. 4), who took a unifying stance to these labels by naming his book "One language, two scripts", comments on these political practises proposing that "[e]thnic groups change into communities and then into nationalities through the efforts of political elites who chose a decisive cultural symbol and manoeuvre other symbols into congruence.” In the same vein, Brass (2005, p. 22) explicitly described the North Indian linguistic division as a result of power abuse by political elites who turned language into 
a symbolic barrier to advance the interests of their religious communities. In the Fijian context, for example, it was not until the politically-driven arrival of religious teachers and missionaries from India had started that the issue of loyalty to Hindi and Urdu scripts came to the fore among Indo-Fijians (Shameem, 1995). Based on this, it is clear that historical events and religiopolitical practices changed the national identity into a religious one. In so doing, political elites used language to make a linguistic border between people, which in its turn contributed to the division between the two language labels. However, this raises some linguistic issues between the so-called two languages. I will now discuss Hindi and Urdu from a linguistic viewpoint, specifically as ausbau languages.

\subsubsection{Ausbau process as language policy and planning in India}

Hindi has different social, regional and standard varieties (Christopher, 1994). While social varieties emerged due to variation in the educational and social levels of speakers, regional varieties evolved under the influence of the regional languages people spoke. Despite these varieties, "it [Hindi] is not exclusively associated with any one region or province"(Shukla, 2006, p. 303). Another form of variation in Hindi is the presence of 'formal' and 'informal' varieties. While informal Hindi is used in everyday speech, formal Hindi is the language of Media, education, and literature (Shukla, 2006). In Hindi, diglossia in the spoken and written varieties is at the lexical level. There are two varieties, high and low, the high variety is often called 'Shudh Hindi' while Hindustani (Hindi-Urdu or Bolcal Hindi) is the low variety (Gambhir, 1995). These varieties and how they developed are illustrated in Figure 1.2.
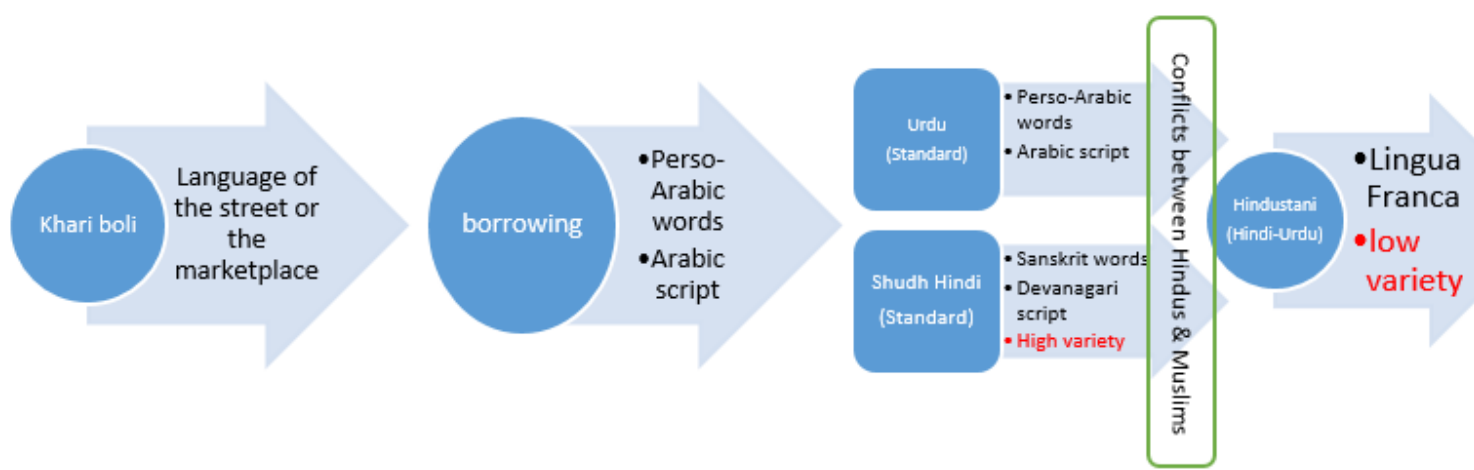

Figure 1.2 Hindustani and the development of Hindi and Urdu

Linguistically, Hindi and Urdu are considered 'one language'. Ammon (2010) referred to the status of Hindi and Urdu as one language with 425 million speakers, ranking second after Chinese. In the same vein, Siegel (1987, p. 139) indicated that "Hindi and Urdu are virtually the same spoken language but differ in standardized written and formal styles". 
However, the religious conflict in India in the beginning of the $20^{\text {th }}$ century, fuelled by the partition of India and independence of Pakistan in 1947, required a solution to the ideologically-fed linguistic problem between Hindi and Urdu. Thus, Indian nationalism movements adopted the traditional language policy and planning (LPP) practices that prominently aimed at "the reduction of diversity" (Mühlhäusler, 2000, p. 306). The 'ausbau process' (Fishman, 1991b), which is a 'planned language reshaping' (Kloss, 1967, p. 33), is responsible for the difference between twin languages such as Czech and Slovak, Danish and Swedish, Bulgarian and Macedonian, and Hindi and Urdu. The term 'Ausbau' (and contrast: Abstand) was first formulated by Heinz Kloss in 1967 (Fishman, 1991b, p. 282) to distinguish between two types of languages; the Ausbau language 'language by development' and Abstand language 'language by distance', with distance understood as intrinsic, but not geographical. Kloss (1967, p. 33) explains that ausbau languages are derived from written forms while abstand languages are based on spoken languages. The emergence of Hindi and Urdu as relying on the written forms of the same spoken language (Hindustani) makes the picture clearer. Further, Kloss (1967, p. 31) described Ausbau languages as follows:

[w]e are dealing with dialects whose speakers would certainly be reported by linguists as constituting a single linguistic community if they were at a preliterate stage. They have, however, created two literary standards which are based on different dialects and therefore characterized by all-pervading differences which, while not necessarily excluding mutual intelligibility, yet make it impossible to treat them as one unit.

In light of this description, it is clear that when a spoken language, in this case, Hindustani, is deliberately written in two writing systems to create 'literary standards', this spoken language will split into two standardized dialects (and then become separate languages). Therefore, it is the systematically planned language reshaping efforts that have made Hindi and Urdu two distinct languages. As mentioned earlier, Hindi and Urdu are different in the standardized written forms of one spoken language (Siegel, 1987). This difference is characterized by the fact that while Hindi utilises the Devanagari script, Urdu utilises the Arabic script (Shukla, 2006). Similarly, Fishman described ausbau language as 'language by effort' whose vocabulary and grammar are similar to an older language. He added that ausbau languages are "built away from more powerful and similar languages so as not to be considered 'mere dialects' of the latter, but rather, to be viewed as obviously distinctive languages in their own right" (Fishman, 1991b, p. 282). Kloss (1967) commented on the possibilities by which planners could enrich the vocabulary of the language. The first possibility is "borrowing from other languages" which is the one used by Urdu language planners by borrowing from Persian, 
Turkish and Arabic. The second possibility is "utiliz[ing] the native 'word-hoard', which can be achieved either by new uses of the morphemes of today's colloquial speech, or by going back to older stages of the language. He exemplified this by noting that "present-day remolders of Hindi go back as far as to Sanskrit" (Kloss, 1967, p. 34).

The difference between 'high forms' of Hindi and Urdu lies in two points (Brann \& Benedict, 1989; Khubchandani, 1989). First, language planners aimed to have a high variety to create a degree of visual distance by distinguishing it visually from its twin sister. While Hindi planners turned to one of the old Indo-Aryan languages (Sanskrit) and adopted one of its scripts (Devanagari), Urdu planners chose to continue with the Arabic transcript which was adopted for literary expression during the Islamic state in North India. Second, patterns of borrowing or 'lexical cleansing' (Trudgill, 2013) for high vocabularies in both languages are different. Whereas Hindi relied on Sanskrit to enrich its high vocabulary, Urdu drew upon Perso-Arabic vocabulary (Shukla, 2006). Khubchandani (1989, p. 601) notes that the phonological and derivational features of the standards were affected by these borrowings. Yet, the common inflectional system, syntax and general vocabulary are still preserved.

Against this background, although Hindi and Urdu are constructed, on the basis of religious divergence, as two distinct languages by 'non-linguistic definitions' (Olphen, 1988), the highly acknowledged mutual intelligibility between their spoken forms gives more room to language users to interact with ease. Yet, using Hindi or Urdu is indexical in Indian Discourse. For example, Ahmad (2007, p. xxiii) in his analysis of language ideologies in North India found that while Urdu indexed education and cultural refinement for the first generation of Indians born before the partition of India in 1947, it became an index to the exclusive Muslim identity for those born after 1947. He also noted that to his third-generation participants, Urdu indexed poor, uneducated and conservative Muslim identity.

Seemingly, this language planning process explains the absence of (Muslim) Indians whose language is socially defined as 'Urdu' from the Hind school in my research. Now I turn to another group of Indians whose language is profoundly linked to Hindi. The following subsection traces the historical development of Fiji Hindi.

\subsubsection{The development of Fiji Hindi}

Fiji Hindi, aka Pidgin Hindustani, evolved from plantation settings (Siegel, 1987). After Fiji had been a British colony in 1874 (Singh, 2008), Fiji attracted Indian labourers during the girmit (work indenture) from 1879 to 1916 (Lal, 2004, p. 28). It was developed as a lingua 
franca for communication purposes (Shameem, 1995) among workers who spoke vastly different languages, dialects and sub-dialects such as English, Fijian, Pacific languages, North Indian varieties (e.g. Bhojpuri, Awadhi, Braj and Rajasthani), and South Indian Dravidian languages (e.g. Telegu, Tamil and Malayalam) (Lynch, 1998). As a result of this linguistic diversity, a pidgin Hindustani (i.e. FH) emerged as a lingua franca among workers and European overseers and then became their children's native language, i.e. FH creole (Shameem, 1995). English vocabulary, such as apul 'apple' and sabal 'shovel' was borrowed through education and the use of English in the community (Siegel, 1987, p. 278). Similarly, Fijian vocabulary was introduced to FH as Fijian was the country's language. Below are some examples of the Fijian influence on FH vocabulary, quoted from Lynch (1998, p. 235).

Fiji Hindi

dakāu

kūmāla

nangona $\bar{a}$

I

you (intimate)

you (formal)

he/she/it (near)

he/she/it (remote) 'reef' 'sweet potato'

'kava'

Fiji Hindi

ham

tum

$\bar{a} p$

$\bar{\imath}$

$\bar{u}$

\section{Fijian source}

cakau

kumala

na yaqona
Hindi dialect source

Bhojpuri ham

Awadhi, Braj tum

Awadhi $\bar{a} p(u)$

Bhojpuro, Awadhi $\bar{\imath}$

Awadhi, Bhojpuri $\bar{u}$

(quoted from Lynch, 1998, p. 235)

The linguistic characteristics of FH are similar to those of Hindi. The only exception is at the lexical level (Lynch, 1998). Among these similarities is the pronoun system as illustrated above, (quoted from Lynch, 1998, p. 235). Furthermore, Indo-Fijians understand Hindi and can speak it fluently on formal occasions, within the media, as a school subject, and as the language of Bollywood (Shameem, 2017, p. 257). Although FH was classified as a creole, a koine and an informally standardized language (Shameem, 1995), it is now considered, by its speakers, as an autonomous language. Indo-Fijian artists started to write literary works in FH using a Romanized FH script (Shameem, 2017).

Fiji and India are separated by geographical boundaries (oceans). FH, as a variety spoken in Fiji, is comprised of forms, sounds and lexicons selected from Fijian, English and other Indian languages. FH could be looked at as (1) a variety of Hindi (because of linguistic links), (2) a 
Fijian variety (because of political, geographical and social links), and as (3) an independent language as per the ideological views of its speakers. In order for $\mathrm{FH}$ to be an independent language, as Trudgill (2013, p. 121) proposes when addressing the Caribbean-English based creoles, it is necessary for FH to achieve independence - autonomy - not only from Hindi but also from other varieties or creoles of the area as well. Unlike other creoles, e.g. the CaribbeanEnglish Creoles and Ullans in North Ireland (See Trudgill, 2013 for details) which have slight mutual intelligibility with the languages they evolved from, FH is substantially mutually intelligible with its lexifier language, Hindi. Thus, there seem to be different views regarding FH. While my Fiji-Indians viewed FH as 'Hindi', Asian Indians tended to report the differences between the two varieties.

Having shown the intertwined linguistic and historical relationship between Hindi, Urdu and FH, I now discuss the status assigned to Hindi in society.

\subsection{Hindi in society}

Hindi is the national language of a multilingual country where the traditional communication norm among people of different linguistic backgrounds is to socialise in Hindi. In this regard, Sridhar (2002) described language use in India, giving the interaction between merchants and customers as an example. To quote Sridhar (2002, p. 267), "[t]he merchants carry on the Indian tradition of making every effort to speak the language of the customers. If they know the language, the exchange is primarily in the customer's language. The second choice is usually Hindi". Sridhar concludes that in the case of merchant customer exchange, "the order of preference seems to be the regional language, Hindi, and lastly, English" (Sridhar, 2002, p. 267). This example suggests that if the merchant were not to linguistically accommodate to the customer's language, he would use Hindi in the interaction as a lingua franca, and although English is a principal language in India, it is the third choice of interaction. This language choice suggests that the language ideologies towards Hindi reflect the people's subscription to the idea of constructing Hindi as 'heritage lingua franca' (Seals, 2013), which is confirmed in this thesis.

It might be suggested this Indian communicative tradition is still extant when members of different Indian ethnic groups meet in diaspora. This is bolstered by studies like Roberts (1999) and Shameem (1995) who indicates that Hindi is part of the respondents' linguistic repertoire. Roberts (1999, p. 217), in her study of the Gujarati community in Wellington, described multilingualism as characteristic of the community, noting that "almost half the respondents 
are trilingual and a handful are quadrilingual. For most people their third language is Hindi”. Furthermore, in a study of three Asian Indian communities in New York, Sridhar (2002, p. 258) maintained that many Indians, such as a Gujarati spice merchant,

may be poorly rated in the area of explicit knowledge of linguistic rules of [the Indian] languages, but in terms of verbal linguistic ability, he [sic] can easily be labelled a multilingual, fairly proficient in controlling different life situations with ease and skill. For this reason, in spite of mass illiteracy, a societal type of bilingualism/ multilingualism [...] has become the life blood of India's verbal repertoire. It is this type of bilingualism that has contributed to language maintenance rather than language shift.

Two points can be inferred from the above quotation. First, Indian multilingualism is conceptually two-fold. On the personal level, it is knowledge of different languages without awareness of the linguistic systems of the languages in the speaker's repertoire. At the societal level, members of Indian wider society are psychologically prepared to experience different languages in various sites (e.g. TV shows, shops, schools). It is worth noting that 'societal multilingualism' is considered one of the reasons that the Indian multilingualism is characterised by 'stability' (Sridhar, 2002). Consequently, Sridhar (1992, 2002) argues that Indian languages tend to be preserved worldwide over generations. In addition, this kind of multilingualism is not related to education. A person, such as the Gujarati spice merchant, might not be skilled at defining the explicit knowledge of the languages s/he speaks. However, this person will still be competent in the oral language skills of most languages. Their linguistic knowledge seems to be implicit and acquired rather than explicit and learnt (Han \& Ellis, 1998). This kind of multilingualism is determined by the instrumental use of these languages. Additionally, the educational system in India maintains a 'Three-Language Formula', according to which "every school-going child learns to read and write in his mother tongue (or the regional language in the case of languages without scripts or literary traditions), Hindi (the official language of the country), and English the associate official language of the country)" (Sridhar, 2002, p. 258). Seemingly, the educational system in India is similar to the concept of flexible multilingual education (Weber, 2014) as it builds on the students' home linguistic resources. It acknowledges the children's native, local and global languages. The existence of this system allows Indians of different linguistic backgrounds to have communicative and literacy knowledge in Hindi. In addition to the Three-Language Formula which promoted the use of Hindi as a second language in non-Hindi speaking regions of India, non-Hindi speakers are exposed to Hindi from Bollywood music and films, as well as internal migration in India (Gambhir \& Gambhir, 2014). This results in potential language use as a lingua franca among 
interactants of different linguistic backgrounds and nurtures the language ideologies towards Hindi in India and diaspora alike.

\subsection{Setting the scene}

The motivation behind choosing the Hindi communities for this study had two parts. First, I had personal experience of conducting research with Indians in Aden-Yemen, which allowed me to gain knowledge about how in that context community languages were able to survive even in the absence of a supportive official language policy. Second, I was drawn in by the complexity and dynamicity of the Indian communities as well as the fact that Hindi speaking communities in New Zealand were not garnering very much attention from researchers or ministries despite their large enrolment numbers. Similarly, the choice of HL in school and home derived from the central role of these institutions in the provision of linguistic input to children (Seals, 2013), as well as being places to build strong rapport with Indian heritage.

The main context of this research was a New Zealand community language school where children of Indian descent learnt Hindi. The HS served as a gateway to the community through which I was connected to teachers, parents, and learners. At the time of this research, the HS consisted of three sites within the same city (A, B, and C) that served the Indian communities in three different suburbs. These sites held their classes in hired facilities. The following is a description of the school site settings.

Site A. This site held its Sunday Hindi classes (from 10 a.m. until noon) in a big single classroom. The classroom had two whiteboards, bookshelves (with a few Hindi books, stories, and periodicals), a cupboard, and U-shaped tables for students to use. This facility was hired from an NGO. In addition to the classroom, there was a lounge where parents waited for their children. Although there were extra rooms on site, the school did not use them as they were booked for other activities. Members also had a kitchen at their disposal. Although the number of enrolled students was around 20 children of Indian descent whose heritage languages included Hindi, Kannada, Telugu and Tamil, only 9-10 students attended the Hindi class regularly. The students were divided into groups according to their language level, and sometimes by age. At the beginning of the school term Sun ji was the only teacher for this site, but later another teacher aide joined. A single teacher on this site created challenges at first as Sun ji had to cope with teaching and administrative responsibilities. An example of these challenges is illustrated in Chapter Four when Sun ji looks for someone to cover her absence 
while overseas. Due to the large classroom space, the school used this venue to hold meetings for all three groups (sites A, B, and C).

Site $\mathbf{B}$ had the highest enrolment numbers and the largest number of students attending the school. It comprised three classes based on the students' levels in Hindi; namely, an entrance, intermediate and advanced class held on Sundays from 10 a.m. until noon. The facilities where these classes took place were hired again from an NGO. There were around 40 enrolled students of Indian descent who spoke different Indian languages, including Hindi, Fiji Hindi, Malayalam, Kannada, and Telugu, and around 20 students attended the classes on a regular basis. The Entrance Class had an average of eight students. They were taught Hindi vocabulary using a textbook in which the Hindi script was accompanied by English transliteration and English meanings. According to the teachers, the students were beginners and had no Hindi literacy skills, but when they moved to the other classes, they were ready to learn to write in Hindi (Fieldnotes 27 August, 2017). These students had been enrolled in the school for three terms at the time of the data collection ( 8 weeks each term). This class also included two Hindi speaking students, but because they were young and recently enrolled, they were temporarily placed in this class. The teacher of this class was the HS principal, Deepika ji, who was a speaker of Fiji Hindi and had received formal teacher training.

The Intermediate Level Class included six frequently attending students. In this class, the students learnt to read and write the Hindi alphabet. All of them were returning students. The teacher of this class, Savita ji, was a native Hindi speaker and received her teacher training at a New Zealand university. In the Advanced Level Class, the average number of students was six. The students in this class enjoyed a good command of Hindi literacy skills (i.e. reading and writing). The teacher of this class, Anita ji, was a New Zealand-born Indo-Fijian who was a former student of the HS. She had a university degree unrelated to education.

Site C. Classes at site C were held on Saturdays from 10.30 until noon. There were around 15 enrolled students, of whom six to nine students regularly attended during data collection. This site had one class that took place in a room hired from a public school. The students were divided into two groups according to their age. The older group (7-years and over) were taught by Sun ji with a special focus on literacy skills (reading and writing), whereas the younger group were taught by a teacher aide, Kaya ji, focusing on speaking and reading stories. 


\subsection{Thesis overview}

With the aim of exploring heritage language learners' identity negotiations and their lived experiences in two key interactional spaces (i.e. HL school and the home), this thesis is comprised of eight Chapters as follows:

Chapter One laid out the foundation for this research. It addressed the research goal and provided a historical account of the Indian communities in New Zealand. Chapter One also elaborated on the historical background of the development of Hindi and related languages with the purpose of showing its current status as the national language of India and the language ideologies accorded to Hindi. This chapter concluded with a description of the Hindi weekend school, the primary site for contacting the community.

Chapter Two presents an overview of language maintenance and language shift research locally and internationally. It describes the status quo of sociolinguistic investigations of HLs in order to situate this thesis within language maintenance (LM) and identity scholarship. Gaps are highlighted and research questions are formulated.

Chapter Three describes the methodology adopted in this thesis, discussing the rationale for adopting ethnography as a research design, data collection methods and procedures, participants, data analysis, and ethical considerations.

The next three chapters present the analyses of the data collected from the HL school, heritage language learners, and the home, respectively. Chapter Four presents the findings of the observed, recorded and reported data within the HS with the purpose of identifying the language practices extant in the school communities of practice (CofPs).

Chapter Five presents analysis of recorded interactions with the students while they undertake a linguistic reflection drawing task. By looking at the influence of the students' membership in the Hindi School CofPs on their representation and negotiation of their multilingual identities, the analysis delves into HL students' multilingual identity development as well as their language ideologies through an indexical lens.

Chapter Six provides a description and analysis of the recordings of home interactions collected in three families with different levels of commitment to the HLs. The language practices within the family were explored to see how they contribute to the construction and negotiation of linguistic, national and cultural identities. Additionally, the intricate relationship between multiple memberships and identity construction is highlighted. 
Chapter Seven discusses the findings reported in the preceding analysis chapters (i.e. Chapters Four, Five and Six) and addresses the research questions. The methodological and theoretical implications are outlined along with the limitations and challenges encountered throughout the course of this research.

Chapter Eight concludes this thesis by summarising the findings of the analyses, suggesting directions for future research and presenting personal reflections on researching with HL speakers/learners. 


\section{Chapter Two: Review of literature}

\subsection{Introduction}

The field of LMLS has recently expanded to incorporate new lines of research, including both family language policy and heritage language education, both of which underpin this study. This expansion has partially resulted from the influence of globalisation which has transformed the world into a global village. Living in an interconnected world has uncovered the complexity and dynamism of the realities people live (Bauman, 1992) and challenged a fixed understanding of, for example, what had been previously understood as language speakers and groups (Blommaert \& Rampton, 2012). Today groups of speakers are characterized by heterogeneity and complex linguistic repertoires (Pauwels, 2016). While previous research likely conceptualised groups as being as less complex than they were in practice, researchers felt justified in making broad and generalised claims. The resulting macro level analysis of individuals' linguistic working abilities contributed to a focus on predicting whether a language was likely to be maintained or otherwise (e.g. Giles, Bourhis, \& Taylor, 1977; Smolicz, 1980). This trend did not allow for explanations which recognise the complex linguistic trajectory of some communities (See Pauwels, 2019 for further discussion). For example, while the analysis of language attitudes is considered an indicator of LM or LS, various studies reported limited language use within the community despite positive attitudes towards the HL (Gharibi, 2016). Similarly, members of HL communities may hold positive attitudes towards their HL, yet identifying with the language may not necessitate speaking it (Dweik, Nofal, \& Al-Obaidi, 2019; Ngaha, 2005). Consequently, there have been increasing calls for micro level analysis to capture the fluidity and complexity of language practices (Blommaert, 2010; Blommaert \& Jie, 2010; Holmes, 1997).

In the New Zealand context, language maintenance and language shift research has been popular since the 1980s. The multilingual and multicultural nature of the country's over 160 languages (The Royal Society of New Zealand, 2013) is well established despite the constantly changing makeup of its cultural diversity. The progressive emergence of Māori nationalism has inspired interest in linguistic and cultural revitalisation of te reo Māori (Albury, 2016; Benton, 1989; Boyce, 1992; De Bres, 2011; Ngaha, 2005; Spolsky, 2005). The revitalisation of te reo Māori has also opened up space for devoting more official and societal efforts to other HLs, exemplified by the increased provision of HLE in state-run schools with no official status 
(Seals, 2017a; Seals \& Olsen-Reeder, 2017) and the support offered to community language schools, though this support is typically symbolic rather than financial.

Te reo Māori revitalisation has similarly enhanced LMLS and HL research in the country. Studies have been conducted with communities originating from Europe (Crezee, 2012; Kuiper, 2005; Neazor, 1991; Plimmer, 1994; Roberts, 2005; Stoffel, 1981), Asia (Adlam, 1987; Kim \& Starks, Jeong Kim \& Starks, 2005; Kim \& Starks, 2010; Roberts, 2005), Africa (Barkhuizen, 2003, 2006; Revis, 2015), Central and South Americas (Lee, 2013; Revis, 2015; Walker, 2011), the Pacific Islands ('Aipolo \& Holmes, 1990; Seals, 2017a; Seals \& OlsenReeder, 2017; Starks, Taumoefolau, Bell, \& Davis, 2005), and the Middle East (Al-Sahafi, 2015; Al-Sahafi \& Barkhuizen, 2006; Gharibi, 2016). Although sociolinguists have been energetic in the field, Hindi has not been a major focus in New Zealand (but see Shameem, 1995 on Fiji Hindi). In recent years, Indian migration to New Zealand has gradually increased, leading to a dramatic rise in the number of speakers of a range of Indian languages. Among these languages, Hindi, which is spoken by over 66,000 speakers, has replaced French as the fourth most spoken language in New Zealand after English, te reo Māori and Samoan (Statistics New Zealand, 2013). This thesis aligns with Blommaert and Rampton's (2012, p. 3) suggestion for sociolinguists to engage with the contemporary social changes associated with superdiversity in relation to the study of language in society. This includes changing the perception of speakers (e.g. HL speakers) and groups (e.g. CofPs) as well as focusing on meaning making rather than form (e.g. indexicality rather than denotational meaning).

In the remainder of this chapter, I discuss major issues that have developed in the field of LMLS and relate these to Indian HLs. This is proceeded by an introduction to HLE locally and internationally. I describe the recent shift of focus in the field from the functional approach, represented by Fishman's key insights, towards considerations of the relationship between HL maintenance and identity, alongside discussions of the CofP model and investment. I then discuss the concepts used in the thesis as a theoretical basis for identity research. Gaps in heritage language research with special attention to the field in New Zealand are introduced towards the end of the chapter.

\subsection{LMLS: Bird's eye view of the field}

Since its inception as a field of inquiry in the mid-twentieth century, LMLS has focused on migrant languages (Fishman, 2013). More recently researchers have turned to local languages (e.g. Gal, 1979; Gorter, Marten, Jansma, \& Ytsma, 1990; Miller, 1971). The scope of LMLS 
has expanded to become a transdisciplinary field engaged in by researchers from the humanities and social sciences, including but not limited to, applied linguistics, education, social psychology, sociology, history and anthropology (See Pauwels, 2016 for further discussion). This expansion encourages the investigation of non-linguistic factors that influence the status of language in contact settings. LMLS factors, whether linguistic or social, are the core of a substantial body of research. Many scholars (Dorian, 1983; Holmes et al., 1993; Kloss, 1966; Weinreich, 1974) list a multitude of factors favourable to language maintenance or language shift, such as numerical power, resistance to mixed marriage and support for community language schools, inter alia. The wider academic focus allows these factors to be central to the investigation.

LMLS literature is enriched by the range of LMLS theories and frameworks, the development of measurements of language endangerment and vitality, and by ongoing methodological and technological advancements. LMLS theories and frameworks have been able to inform research to "predict whether a group is likely to maintain its language" through "identifying which particular combination of factors can speed up or reduce the process of LS" (Pauwels, 2016, pp. 100-105). Dominant approaches include: The ecology approach (Haugen, 1972) which views language as a dynamic and holistic system (Eliasson, 2015) that connects various linguistic subfields, e.g. historical and descriptive linguistics, sociolinguistics, dialectology and others (Haugen, 1972); the core value theory (Smolicz, 1980) sheds light on values and beliefs (e.g. religious and familial) and their impacts on differences in LMLS patterns among ethnic groups; ethnolinguistic vitality (Giles et al., 1977) seeks to understand "the interrelationship among language, ethnicity and intergroup relations" (Giles et al., 1977, p. 308) from a social psychological perspective; social network theory (Milroy, 1980) has shifted the focus of LMLS research to an interactionist approach in order to understand the complexity of LMLS patterns (Pauwels, 2016); social mobilization theory (Paulston, 1994) centres social conditions to account for the reasons why ethnic groups vary in terms of language contact outcomes. Although these theories are contested by scholars, they have contributed to a better understanding of the dynamics of the field. In line with LMLS theories, various measurements of language endangerment and vitality, e.g. Fishman's (1991a) Graded Intergenerational Disruption Scale (GIDS), UNESCO's (2003) vitality factors, and Lewis and Simons' (2010) Expanded Graded Intergenerational Disruption Scale (EGIDS) have furnished solid ground for researchers to delve into relevant factors, especially at the group level, and to assess the degree of language shift and endangerment. 
Furthermore, the significant methodological and technological advancements that the world has witnessed in the past two decades have illuminated our understanding of the dynamic nature of the field. New subfields have emerged including Reverse Language Shift (Fishman, 1991a), Family Language Policy (King, Fogle, \& Logan-Terry, 2008) and Heritage Language Education (Brinton, Kagan, \& Bauckus, 2008). Audio and video recording devices have allowed for deeper levels of investigation, e.g. recording of naturally occurring interaction at home, HL school and workplace. Other non-scholarly factors, such as extensive migration, changes in political stances and globalisation, have also contributed to the study of LMLS because of their impact on individuals, groups and societies.

These advancements and changes have led researchers to conduct studies in different parts of the world and from a multitude of perspectives. While some researchers focus on macro levels of investigation (i.e. the sociology of language) such as language proficiency (Christensen, 2003; Gharibi, 2016; Roberts, 1999) language attitudes and language use (Bissoonauth, 2011; Crezee, 2012; Nofal, 2011; Plimmer, 1994) in different domains (e.g. home, place of worship, workplace), others prefer micro issues such as interaction either at home or elsewhere (e.g. Hay, 1994; Yakushkina \& Olson, 2017), and identity (e.g. Barkhuizen \& De Klerk, 2006; Seals, 2017b) which will be discussed later in this chapter. The complexity of the field is well illustrated by Pauwels (2016, p. 100) when she pointed out that the most consistent finding in LMLS is that LMLS rates differ from one group to another, but also that these rates differ for the same group across contact contexts.

The main LMLS theories and frameworks overlap in the sense that they share the same objective with different nuance. Arguably, they all originally derive from Fishman (1966, p. 424) who foregrounds three major sociological elements of the field as follows:

1. The habitual use of language at more than one point in time or space under conditions of intergroup contact.

2. The antecedent, concurrent or consequent psychological, social and cultural processes and their relationship to stability or change in habitual language use.

3. The behavior toward language in the contact setting, including directed maintenance or shift efforts.

The first element accounts for language use in terms of degree, location and direction of bilingualism within the language community. This involves the exploration of language background and proficiency through an official census or others means (Garcia, 2003; Holmes, 1997; Pauwels, 2016). A stream of research studies use various ways of gauging the linguistic 
competence of the respective community members. While for many researchers the common practice is to rely on respondents' reported language proficiency, i.e. surveys and interviews, (e.g. Al-Sahafi \& Barkhuizen, 2006; Boyce, 1992), others rely on parents' reporting of their children's proficiency (e.g. Roberts, 1999). Some validate report results by means of a language performance test (e.g. Shameem, 1995), and recently Gharibi (2016) performed a Verbal Fluency Test (VFT) and auditory picture-word matching test (APWMT) to ascertain the vocabulary knowledge of heritage speakers of Persian in New Zealand. Data surrounding language use is typically collected quantitatively and/or qualitatively. The qualitative collection of language use data embraces interviews (Perera, 2015), focus groups (Mirvahedi, 2014), case studies (Walker, 2011), observations (Gal, 1979), narratives (Barkhuizen \& De Klerk, 2006), diaries (Jeong Kim \& Starks, 2005), autobiographies (Alatis \& Tan, 1999) and correspondence (Folmer, 1992). In contrast, quantitative data collection methods include using census data, questionnaires, interviews and proficiency tests, as mentioned above (See Pauwels, 2016, for further discussions).

The second sociological element elaborates on the factors that "are primarily OUTSIDE the language per se" (Fishman, 1964, p. 49, emphasis original). These non-linguistic factors influence language maintenance or language shift and are heterogeneous according to the location of the bilingualism, i.e. domains or "occasion on which one language (variant, dialect, style, etc.) is habitually employed rather than (or in addition to) another" (Fishman, 1966, p. 428).

Finally, the third element of the field represents attitudes towards a language on the part of its speakers, other groups and the presence or absence of language reinforcement and language planning in the form of voluntary and/or "official organizational protection, statutory protection, agitation and creative production" (Fishman, 1966, p. 450). Although positive attitudes contribute to the continuity of a language (Baker, 2006), these positive attitudes do not guarantee language maintenance (Nguyen \& Hamid, 2016) unless they are "conducive to an active effort to maintain the [HL]" (Cherciov, 2012, p. 730).

\subsection{Multilingualism and LMLS}

Fishman (1989) proposes three potential outcomes resulting from languages in contact. $\mathrm{He}$ points out that some immigrant languages come to dominate the host language, like English in the United States and Canada (Dweik, 1985). The second outcome is the immigrant language being dominated by the host language, as is the case of some ethnic languages in the Arab 
world (e.g. Rannut, 2009). The third involves a diglossic relationship between the host country's dominant language and the immigrant languages, where each is used in certain domains (e.g. Dweik, 2000). Describing the same results, Paulston (1994) and Romanov (2000) use LMLS terminology, i.e. language maintenance, bi- or multilingualism and language shift. Fishman (1989, p. 206) laments that "what begins as the language of social and economic mobility ends, within three generations or so, as the language of the crib as well, even in democratic and pluralism-permitting contexts". Fishman's claim connotes language shift as an inevitable outcome of social and economic activities of ethnic communities, turning a previously foreign language into a native one within almost three or 'four generations' (Holmes et al., 1993). Likewise, findings in diaspora related research regularly show that ethnic groups are susceptible to abandoning their HLs by the third generation (Lasagabaster, 2008; Porcel, 2006; Totoricaguena, 2003).

However, this pattern has not gone unchallenged. Researchers rallied to test the validity of Fishman's claim in a range of languages. One of the most explicitly opposing proposals is Sridhar's $(1985,1992 ; 2002$, p. 258) who argued that "speakers of Indian languages tend to maintain their languages over generations and centuries, even when they live away from the region where it is spoken". Sridhar's statement indicates that bilingualism, as the threshold of language shift, does not hold true in the Indian context. In the same vein, although Paulston (1994, p. 13) points out that "bilingualism is a mechanism of LS", there is a different viewpoint for Indian bilingualism. Sridhar refers to Indians as an exceptional example of "maintained group bilingualism" that is conducive to language maintenance. While bi- or multilingualism can be seen as a "mechanism of language shift" (Paulston, 1994, p. 13), this is only when it is in its "transition state" (Kandler, Unger, \& Steele, 2010). Bi- or multilingualism may in fact be an effective path for language maintenance if it is in the "final stable state" (Kandler et al., 2010). Bi- or multilingualism per se is not what LM requires, but rather it is "stable societal bilingualism" (Kandler et al., 2010, p. 3858) that achieves LM. In this regard, Sridhar (2002) attributes maintenance of Indian languages to stable societal multilingualism.

As the HS is a primary site for data collection in this thesis, the following section sheds light on the field of heritage language education.

\subsection{Heritage language education}

HLE is an emerging field concerned with the acquisition and maintenance of HLs, the expansion of bilingual range, and the transfer of not only oral language abilities, but also 
literacy skills (e.g. reading and writing) across languages (Gharibi, 2016; Valdés, 2014). Although HLE has been practised for a long time (Fishman, 2014 [2001]), not till recently has HLE received attention as a field with its own pedagogical approaches (Hornberger \& Wang, 2008). Recent scholarship has shown the value of HLE for individuals and society (Brinton et al., 2008; Hallett, Chandler, \& Lalonde, 2007; Hornberger \& Wang, 2008; Seals, 2017a; Seals \& Peyton, 2016). Campbell and Rosenthal (2000) and Wang and García (2002) promote the understanding that HLs are national resources that must be preserved and developed to build foreign language capacity (of the USA in this case). Wang and García further argue that HLE enables HL students to build healthy identities whereby they negotiate their place in their heritage community and the wider society $(2002$, p. 4).

The fact that HL groups are often heterogeneous populations who do not share a specific linguistic or ethnic background has led to a blurred picture of who HLLs are (Wang \& García, 2002). However, researchers aim to define and describe HLLs (e.g. Draper \& Hicks, 2000; Fishman, 2014 [2001]; Kelleher, 2010; Valdés, 2001). Hornberger and Wang (2008, p. 6) view heritage language learners as "individuals with familial or ancestral ties to a language other than [the dominant societal language] who exert their agency in determining if they are [heritage language learners] of that language." This definition highlights an individual's investment in learning the language, but also differentiates between HLL and heritage language speakers who are "native speakers of a minority language they learn at home, but due to sociopolitical pressure from the majority language spoken in their community, their heritage language does not fully develop" (Montrul, 2016, p. i). This has led to extending the scholarly discussion over these terms. Recently, Seals and Shah (2017, p. 3) have defined heritage language speakers as

people who have a recent or ancestral connection to a language that is not the dominant societal language in their current region of residence. Furthermore, heritage language speakers use their agency to identify with the heritage language(s). Heritage language speakers may be at any level of proficiency. Additionally, a person may still identify with (a) particular heritage language(s) based on their personal background, without having to actually have proficiency in the language(s). Finally, given the importance of identity and agency in determining heritage languages, heritage languages include immigrant, Indigenous, and ancestral languages of either kind.

Seals and Shah's definition is inclusive of both learners and speakers since it considers the individual's agency and self-identification. This way, Seals and Shah emphasize the individual's self-positioning in relation to HL(s) rather than how they are positioned by others. Regarding HLLs of Hindi, Gambhir and Gambhir (2014) note that Hindi HLLs in the United 
States are either 'ancestral' or 'associate' learners. While the former are those who come from Hindi-speaking Indian families, the latter come from non-Hindi-speaking Indian families (Gambhir and Gambhir, 2014, p. 208).

In the New Zealand context, despite the many calls for developing a national languages policy whereby community languages are included in education (e.g. New Zealand General Election, 2014; The Royal Society of New Zealand, 2013; Waite, 1992), there is still no official languages policy, with some notable exceptions (See Seals, 2017a). Significant steps have been taken towards supporting some community languages, including te reo Māori, New Zealand Sign Language (NZSL), Pasifika languages and Mandarin (De Bres, 2015; Seals, 2017a; Seals \& Olsen-Reeder, 2017). Governmental support for New Zealand community language takes different shapes and varies from dedicated support for de jure languages (i.e. te reo Māori and NZSL) to unsupported languages such as Ukrainian and Arabic (Seals \& Olsen-Reeder, 2017).

Hindi is one of the unrecognized community languages in New Zealand and receives no support in formal education, despite having the fourth largest number of speakers in the country. In the 2013 Census, 143,520 people identified as Indian alongside an additional 10,929 Fiji Indians. The census data also shows that Hindi is the second-most common language spoken among the Indian population, of whom over 66,000 identify with Hindi (Statistics New Zealand, 2013). However, providing Hindi education is seen as the community's responsibility. The Hindi communities in New Zealand operate HL schools on a voluteer basis in cities such as Auckland, Wellington, Christchurch and recently Invercargill. All community-based Hindi schools have a framework and guidelines to work with. Some have engaged experts and developed their own curriculum to suit the New Zealand environment while setting general educational standards (Narayan, 2015a). Community school resources such as books are obtained from India and Fiji while the school sometimes develops some very basic resources to supplement the printed material sourced from home countries. Hindi schools in New Zealand receive occasional support from the Indian government, represented by the Indian High Commission through the donation of books and small pots of funding (Narayan, 2015b). Support from the New Zealand government comes from the Office of Ethnic Communities which is part of Department of Internal Affairs (DIA) in the form of contestable funding for cultural activities such as Hindi language week. In general, governmental support for HLs is not based on numbers of HL speakers. It varies according to other factors, for example, the socio-economic situation of the group and the support the group receives from 
language and cultural organisations in negotiations (De Bres, 2015; Holmes, 1997; The Royal Society of New Zealand, 2013).

Against this background, Chapter Four of this thesis provides a profile of how HL schools operate when left 'high and dry' without dedicated institutional support beyond the community itself, addressing languages practices in HLE of Hindi in a New Zealand weekend Hindi school through the CofPs analytical model (which is discussed in the next section).

\subsection{Communities of practice and investment}

Originally proposed in Lave and Wenger's (1991) work around situated learning and developed further in Wenger (1998b) and Wenger, McDermott, and Snyder (2002), communities of practice are defined as "groups of people who share a concern, a set of problems, or a passion about a topic, and who deepen their knowledge and expertise in this area by interacting on an ongoing basis" (Wenger et al., 2002, p. 4). The term is used in a plethora of disciplines including education, applied linguistics and sociolinguistics. The concept of CofPs was adopted into sociolinguistic research via Eckert and McConnell-Ginet's (1992) study of language and gender. Eckert and McConnell-Ginet call upon the need for sociolinguists to have "a conception of a community that articulates place with practice" (1992, p. 464) where membership is defined by participation in the shared practice (Eckert, 2000). It has also been used in other sociolinguistic areas such as leadership and workplace research (Angouri, 2018; Holmes \& Marra, 2002; Holmes, Marra, \& Vine, 2011; Schnurr, Marra, \& Holmes, 2007; Vine \& Marra, 2017), identity studies more generally (Bucholtz, 1999; Mendoza-Denton, 1997; Wilson, 2011), and importantly heritage language research (Lyutykh, 2011; Oriyama, 2016; Revis, 2017; Weldeyesus, 2009). Bringing the concept of CofP to their research has enabled sociolinguists to add an additional analytical tool to their understanding.

According to Eckert and McConnell-Ginet (1992), one of the reasons for adopting the CofP framework into sociolinguistic research was the difference between CofPs and other sociolinguistic concepts such as 'speech community' (Labov, 1972), the in-groups of social identity theory (Tajfel, 1978), and 'social networks' (Milroy, 1980).

Eckert and McConnell-Ginet (1992, p. 464) offered a tailored definition:

an aggregate of people who come together around mutual engagement in an endeavor. Ways of doing things, ways of talking, beliefs, values, power relations-in short, practices-emerge in the course of this mutual endeavor. As a social construct, a community of practice is different from the traditional 
community, primarily because it is defined simultaneously by its membership and by the practice in which that membership engages.

In speech communities, members share a set of evaluations about the speech of that community, e.g. standard forms are prestigious (Labov, 1972). In contrast, in CofPs people's engagement in different activities generates shared knowledge. That is, the shared practice is a by-product of interaction rather than a base for interaction. Moreover, since people come together for a shared endeavour, they get to know one another and build up relationships based on their interaction in the CofP. The CofP does not require the (implicit) homogeneity found in speech communities, nor does it focus on the quantity of interaction in the way that a social networks model does (Holmes \& Meyerhoff, 1999). Rather, membership in a certain CofP is defined by partaking in the shared practice (Eckert, 2000) that is conducive to the shared goal, regardless of whether members share the same linguistic or cultural norms outside of the CofP (See Bethan Davies, 2005; Holmes \& Meyerhoff, 1999, for further discussion). In sum, CofPs combine the 'shared norms' concept from speech communities and the on-going interaction of social networks as tools to achieve a 'joint enterprise' (Wenger, 1998b). Holmes and Meyerhoff (1999) summarise the key differences between speech community, social identity, and CofP models (to which I add the social networks column for comparison purposes) in Table 4.1.

Table 4.1 Comparing speech communities, social identity, social networks, and CofP, adopted and modified from Holmes and Meyerhoff (1999, p. 179) with social networks added to the original table.

\begin{tabular}{|c|c|c|c|}
\hline Speech community & Social identity & Social network & Community of practice \\
\hline $\begin{array}{l}\text { Shared norms and } \\
\text { evaluations of norms } \\
\text { are required. }\end{array}$ & $\begin{array}{l}\text { Shared identifications } \\
\text { are required. }\end{array}$ & $\begin{array}{l}\text { Social ties, e.g. } \\
\text { kinship, are required }\end{array}$ & $\begin{array}{l}\text { Shared practices are } \\
\text { required. }\end{array}$ \\
\hline $\begin{array}{l}\text { Shared membership } \\
\text { may be defined } \\
\text { externally. }\end{array}$ & $\begin{array}{l}\text { Membership is } \\
\text { constructed internally } \\
\text { or externally. }\end{array}$ & $\begin{array}{l}\text { Membership is } \\
\text { constructed internally } \\
\text { or externally. }\end{array}$ & $\begin{array}{l}\text { Membership is internally } \\
\text { constructed. }\end{array}$ \\
\hline $\begin{array}{l}\text { Nothing to say about } \\
\text { relationship between } \\
\text { an individual's } \\
\text { group and personal } \\
\text { identities. }\end{array}$ & $\begin{array}{l}\text { Relation between } \\
\text { group and personal } \\
\text { identities is unclear: } \\
\text { continuum? } \\
\text { orthogonal? }\end{array}$ & $\begin{array}{l}\text { Relation between } \\
\text { group and personal } \\
\text { identities can be either } \\
\text { compatible or } \\
\text { otherwise }\end{array}$ & $\begin{array}{l}\text { Actively constructed } \\
\text { dependence of personal } \\
\text { and group identities. }\end{array}$ \\
\hline Non-teleological. & $\begin{array}{l}\text { Non-teleological: any } \\
\text { outcomes are } \\
\text { incidental. }\end{array}$ & Non-teleological & $\begin{array}{l}\text { Shared social or } \\
\text { instrumental goal. }\end{array}$ \\
\hline $\begin{array}{l}\text { Nothing to say about } \\
\text { maintenance or } \\
\text { (de)construction }\end{array}$ & $\begin{array}{l}\text { Group identity is } \\
\text { defined through } \\
\text { comparison and }\end{array}$ & $\begin{array}{l}\text { Nothing to say about } \\
\text { maintenance or } \\
\text { (de)construction of }\end{array}$ & $\begin{array}{l}\text { Boundaries are } \\
\text { maintained but not }\end{array}$ \\
\hline
\end{tabular}




\begin{tabular}{llll}
$\begin{array}{l}\text { of boundaries } \\
\text { between categories. }\end{array}$ & $\begin{array}{l}\text { competition with } \\
\text { outgroups. }\end{array}$ & $\begin{array}{l}\text { boundaries between } \\
\text { categories. }\end{array}$ & $\begin{array}{l}\text { necessarily defined in } \\
\text { contrasts-with outgroups. }\end{array}$ \\
$\begin{array}{l}\text { Acquisition of } \\
\text { norms. }\end{array}$ & Learning incidental. & $\begin{array}{l}\text { Nothing to say about } \\
\text { learning. The focus is } \\
\text { on quantity and } \\
\text { quality of interaction }\end{array}$ & Social process of learning. \\
\hline
\end{tabular}

The CofP model has been a helpful tool in illuminating the dynamics of interaction in different contexts, including heritage language education. As an example, Lyutykh (2011, p. 25) describes weekend Russian schools in the USA as dynamic CofPs, because they bring together the parents, the children, and the educational context to create spaces for practising the Russian language and traditions. To put it in Fishman's terms (1965), weekend schools help create a supportive 'domain of language use' that is suitable for HLL. In recent research on a Japanese community in Australia, Oriyama (2016) directly connects communities of practice to HLL, adding an ideological component to the definition. Oriyama defines HL communities of practice as

socially and closely networked HL groups with common ideology: values, attitudes, norms, knowledge, and goals (e.g., HL development and maintenance, pursuing interest in HL culture) who regularly and actively contribute to, and engage in, shared practices and learning to achieve their goals, often through HL community schools and associations" (2016, p. 292, emphasis mine).

Oriyama highlights the contribution of the community language school (as an institutional space for a CofP) to language ideologies and practices among members of the community. Furthermore, because immigrant families are often scattered across cities, participation in community language schools is a common way to exert agency and self-identify with the heritage language and culture, which is represented through people's involvement in the cultural community of practice (Lyutykh, 2011, p. 26). Thus, HL schools are seen as a midpoint between marginalisation (where languages are alienated) and institutional recognition (where languages are available in formal education). Community language schools are understood as not only ensuring HL literacy but also being a transforming point for the language to gain official status. In other words, by having a community language school, the community has a better place to negotiate the position of their language in society (De Bres, 2015).

HL schools are often considered to be CofPs since community member share the same interest, but does this mean that HL schools can automatically be considered CofPs? In 2005, the Council of Europe Framework Convention on the Value of Cultural Heritage for Society introduced 'heritage community' as a group of “people who value specific aspects of cultural 
heritage which they wish, within the framework of public action, to sustain and transmit to future generations" (Council of Europe, 2005, p. 2). Against this backdrop, Adell, Bendix, Bortolotto, and Tauschek (2015, p. 8) suggest that heritage communities are no longer viewed as "bearers" of heritage, but viewed as 'heritage communities of practice' responsible for developing and transmitting heritage. This view of HL communities as CofPs has partially led to a loosening of the concept of CofP within the subdiscipline to fit these communities, including glossing over the complexities of these communities and the 'mutual engagement' criterion amongst its members (See also King, 2019, for critical review).

Although the term CofP is based on an emergent dynamic practice-based approach, some researchers have adopted a fixed approach to viewing these schools as CofPs. In so doing, they may unconsciously treat HL schools as automatically presenting as CofPs without paying attention to the dynamism of these schools (e.g. Lyutykh, 2011; Oriyama, 2016). In fact, HL schools are dynamic and often superdiverse. HL schools can also include different sites and classes where each of these sites/classes can form a separate CofP (as is the case with the HS). It is noteworthy that location of school sites/classes does not predetermine their distinct status. Rather, what makes an aggregate of people a CofP is the existence of Wenger's (1998b) indicators, which can be used to identify whether a CofP exists (Holmes \& Meyerhoff, 1999). According to Wenger (1998b, pp. 125-126), these indicators are:

- Sustained mutual relationships-harmonious or conflictual

- Shared ways of engaging in doing things together

- The rapid flow of information and propagation of innovation

- Absence of introductory preambles, as if conversations and interactions were merely the continuation of an ongoing process

- Very quick setup of a problem to be discussed

- Substantial overlap in participants' descriptions of who belongs

- Knowing what others know, what they can do, and how they can contribute to an enterprise

- Mutually defining identities

- The ability to assess the appropriateness of actions and products

- Specific tools, representations, and other artefacts

- Local lore, shared stories, inside jokes, knowing laughter

- Jargon and shortcuts to communication as well as the ease of producing new ones

- Certain styles recognized as displaying membership

- A shared discourse that reflects a certain perspective on the world.

Drawing upon the underlying premise that CofPs represent learning as a social process, Trentman (2013) suggested that CofPs influence the learners' investment in language learning by granting them legitimacy or 'the right to speak' (Norton Peirce, 1995) as they move from 
the periphery to full participation. 'Investment' was introduced by Norton (Peirce) in the 1990s and defined as "the socially and historically constructed relationship of learners to the target language, and their often ambivalent desire to learn and practice it" (Norton and Toohey (2001, p. 312). This suggests that in order for learners to develop desired identities, they need to access symbolic and material resources. Norton was inspired by Bourdieu's $(1977,1990,1991)$ work, in particular the notion of cultural capital which refers to "the knowledge and modes of thought that characterize different classes and groups in relation to specific sets of social forms" (Bourdieu \& Passeron, 1977 cited in Norton Peirce, 1995, p. 17). "Investment" has served to explain inconsistenies between the concept of motivation and findings of research in the field of second languge acquisition (Norton, 2013). Investment also problematizes the traditional conceptionalisation of a language learner's identity and recognises that power relations in different contexts can position the learners in multiple and often unequitable ways. This in turn explains why some (motivated) learners may not feel comfortable to speak the language (Darvin \& Norton, 2015; Norton, 2013). Thus, Norton suggests that learners, as social beings with multiple identites, are committed to language learning to gain access to hitherto unattainable symbolic (e.g. language, education, friendship) and material resources (e.g. capital good, money) that increase the value of their cultural capital and social power, but also develop their own social identities (Norton, 2013; Norton Peirce, 1995). Recently Darvin and Norton (2015) developed a model of investment that looks beyond "the microstructures of power in specific communicative events" while investigating "the systemic patterns of control that communicative events are indexical of" (p. 42). The model includes not only identity, but also ideologies and capital. Identity is defined as multiple, a site of struggle, and continually changing over time and space. The model explains identity further as a struggle of habitus and desire, of competing ideologies and imagined identities (Darvin \& Norton, 2015, p. 45). The model also recognizes the importance of ideology as a complex and layered construct that regulates the positioning of learners in linguistic exchange. Thus, ideologies as a polylithic concept references the "dominant ways of thinking that organize and stabilize societies while simultaneously determining modes of inclusion and exclusion" (Darvin \& Norton, 2015, p. 44), and supplements the shifting value of capital across contexts. As for capital, Norton adopts Boudieu's notion of forms of capital to relate investment to identity, explaining that

capital is power and it extends from the material/economic to the cultural and social: Economic capital refers to wealth, property, and income; cultural capital refers to knowledge, educational credentials, and appreciation of specific cultural forms; and social capital refers to connections to networks of power. The value of these forms of capital is determined by ideological 
structures, but it is continually negotiated in different fields or sites of struggle. (Darvin \& Norton, 2015, p. 44, emphsis original)

Norton (2013) connects learners' identity claims to investment by means of the value assigned to capital in different contexts, and borrows Bordieu's (1987) notion of 'symbolic capital' to demonstrate the fluidity of capital across time and space. The concept of investment is central to this research as it helps identify the relationship between HLLs and their lived experiences as well as the negotiation and development of their identities when participating in the HS and community practices. Now I turn to the discussion of the relationship between language and identity.

\subsection{Shifting focus: Language and identity}

In the first section of this chapter, I discussed the functional approach to LMLS research. This section overviews recent trends in the field, shifting focus from HL working abilities to the way in which people perform their various identities through language use and identification.

\subsubsection{Identity and interaction}

Identity has been a focus for a wide spectrum of disciplines, including psychology (e.g. Burke, 2009; Cote \& Levine, 2014), sociology (e.g. Bandyopadhyay, 2010; Rutherford, 1998; Tracy, 2002), language learning and teaching (e.g. Cook, 2008; Day, 2002; Norton, 2000, 2010), and linguistics (e.g. Bucholtz \& Hall, 2004; Bucholtz \& Hall, 2005; De Fina, 2015; De Fina \& Perrino, 2013; De Fina \& Tseng, 2017; King, 2013).

Identity is no longer seen as "a stable structure located primarily in the individual psyche or in fixed social categories" (Bucholtz \& Hall, 2005, p. 586), nor is it seen as sets of characteristics that can be ascribed to individuals (De Fina, 2015, p. 351). Rather, identity or its preferred plural form 'identities' (De Fina \& Tseng, 2017) are dynamic and multi-faceted (Hua, 2017; Skutnabb-Kangas, 2000), and are constituted in and through linguistic interaction and through semiotic processes (Bucholtz \& Hall, 2005; De Fina, 2015; Tracy \& Robles, 2013). For Kroskrity (1999a, p. 111), identity is defined as "the linguistic construction of membership in one or more social groups or categories". Kroskrity's definition asserts the importance of language as a means of identity construction. De Fina, Schiffrin, and Bamberg (2006, p. 2) emphasise the central role of discourse practices in identity construction which take place within social practices. Similarly, De Fina (2015) states that identities emerge in order for people to construct images of themselves and others, and Bucholtz and Hall (2005) illustrate this emergence of identity by providing evidence from interactions of speakers using discourse 
to construct identities that are different from the identities with which they phenotypically appear. This is exemplified in Bucholtz and Hall's illustration of hijras who use feminine forms to refer to themselves so as to be at odds with the masculine identities whereby their families refer to them, or young Asian Americans who draws on African American Vernacular English (a variety they do not normally use) to show an antiracist identity and solidarity with non-white Americans.

The pivotal discrepancies between the essentialist and social constructivist views of identity are related to the nature of identity. The former views identity as innate, stable and unchanged (cf. May, 2012; Omoniyi \& White, 2006). Conversely, the latter promotes the dynamic, fluid and socially constructed nature of identity (Beswick, 2007; May, 2004, 2012). Through the lens of social constructionism, identity is viewed as a social process emerging within discursive practices, and brings about a focus on the existence of the 'other' for identity to be constructed. In other words, as identity enactment occurs in interaction, which in turn takes place within social settings between two or more interlocutors, it is what we receive from other interactants that shapes how we respond and what stance we take and consequently what identities we perform (Fuchs, Linkenbach, \& Malik, 2010). In line with this, Hua (2017, p. 117) suggests that "identities entail the juxtapositions between the self and the other". Hua's suggestion highlights the role of the interactant in one's identity construction. That is, the identities one might enact in interaction are not always predetermined, but depend on, for example, the way that person is positioned by their interactants. The bidirectional nature of identity enactment is well-articulated when we understand interactants as choosing the discursive practices that "shape who he or she is taken to be and who the partner is taken to be." (Tracy \& Robles, 2013, p. 26). An illustration of how others influence one's identity is shown by Seals (2017b) in her study of heritage language speaking children and how they are discursively positioned by parents and teachers and its contribution to their own multilingual identities. Chapter Six of this thesis also provides examples of how discursively assigned identities lead to resistance, rejection and negotiation of these identities. The dynamic nature of identities can be illustrated by the 'repertoires of identity' (Kroskrity, 1999b) an individual has at their disposal and can assume or abandon as required (Beswick, 2007, p. 45). Such repertoires includes "contradictory identities [that] may coexist within the same individual" (De Fina, 2015, p. 352) and can be distinguished from the sets of characteristics an individual has by (1) the fact that they are 'communicatively produced' and (2) the contradictory identities an individual has. Thus, as Kroskrity notes in the definition above, the key role of language in constructing who 
we are makes a difference between the essentialist and social constructionist views of identity. Identities also mirror the complex multiple and dynamic group memberships (e.g. ethnic, social or professional) which are not part of one's stable characteristics. Individuals can have contradictory identities but not contradictory characteristics. These contradictory identities appear in their speech as they correspond to their interactants. For instance, one can't be 'generous and miser' or 'happy and sad' at the same time, but they can show their association and dissociation with a certain group simultaneously as in the examples in Bucholtz and Hall (2005) when a girl showed in-groupness and then distanced herself from the same group discursively.

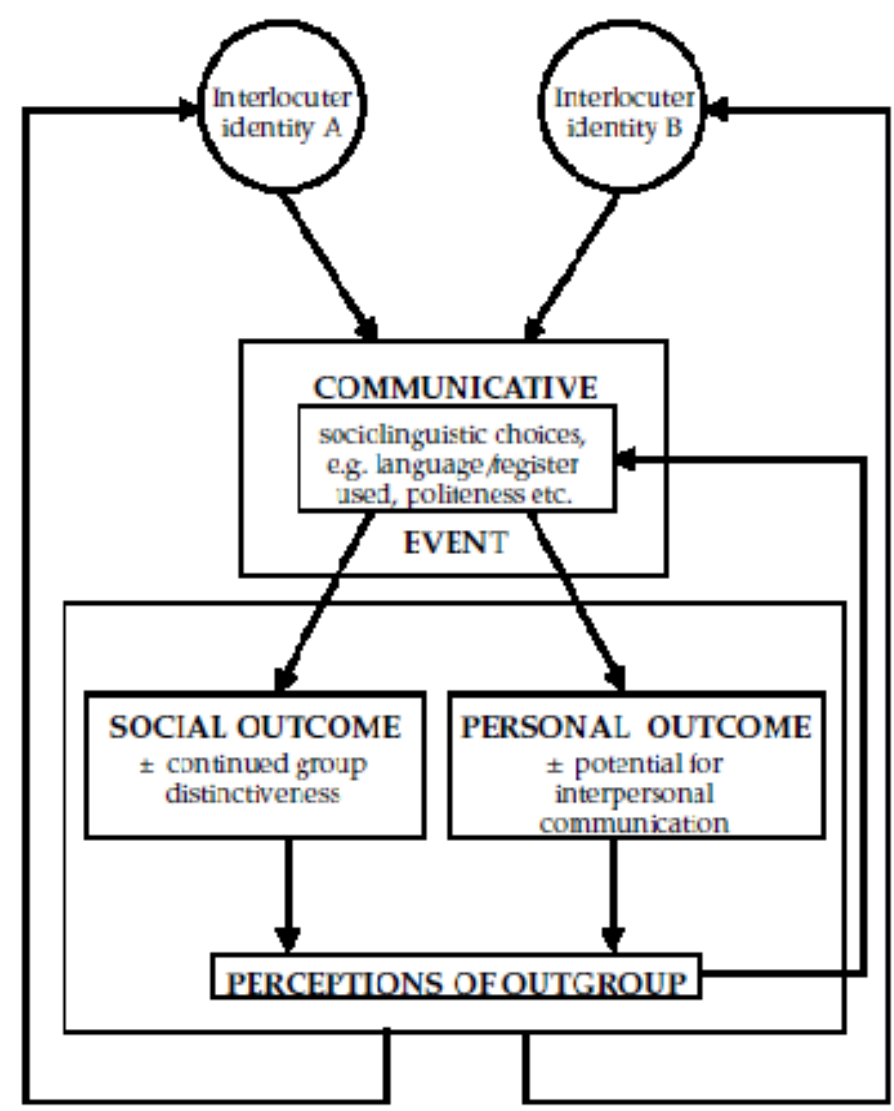

Figure 2.1 Model of a communicative event (quoted from Holmes, 1997, p. 30)

In an attempt to show how individuals are constantly constructing their identities and social roles (as a working woman, as a mother, as a friend, as a young person), in daily interaction, Holmes (1997) developed a model for interaction that serves as a framework to consider the construction of ethnic identity through language choice. Holmes' model in Figure 2.1 visualizes how ongoing interaction passes through different stages from sociolinguistic choices (e.g. register, politeness, language variety) to the social or personal outcomes the speakers wish 
to get (i.e. they identity they do), and then responding to the other's social or personal outcomes through sociolinguistic choices (Holmes, 1997, p. 30) ${ }^{3}$.

Indeed, identity negotiations occur in interaction when individuals (dis)align themselves between prescription-by-others and self-orientation (Hua, 2014, p. 216). In this regard, King's (2013, p. 61) study of a transnational family showed how language identity prescribed by other family members can shape language competence. In King's study of three siblings, the elder sibling was framed by the other siblings as the unsuccessful English language learner (because her first language was Spanish), whereas the younger sister was framed as a problematic Spanish speaker (as she grew up in the United States) but also as a proficient English user. The youngest was framed as the English monolingual, although she was a baby who had not begun to speak yet.

\subsubsection{Identity in mobility}

Identity in mobility research, i.e. with migrants, has illuminated the understanding of the complexity of identity. A stream of research refers to hybridity (or double belonging) among migrant communities (Hussain, 2019; Pavlenko, 2001; Piller, 2002; Williams, 2010). Hybrid identities "describe the mix and plurality of identity that results from the phenomenon of togetherness-in-difference" (Hua, 2017, p. 118) and combines the 'roOts' and 'roUtes' categories of ethnicity (Harris \& Rampton, 2003). 'RoOts' and 'roUts' ethnic identification reflects the fluid and situational nature of ethnic identity whereby individuals or groups associate with or dissociate from groups or institutions according to their needs and purposes in context (Harris \& Rampton, 2003, p. 5). This kind of hybridity or multiple identification is expressed by hyphenated denominations which "[are] mostly applied to second- or thirdgeneration ethnic minorities" (Raghunandan, 2012, p. 7) such as Afro-American, Fiji-Indian and 'Kiwi-Indian' (Bandyopadhyay, 2010). On the one hand, using hyphenated terms, e.g. FijiIndian, associates this specific group of people to their ancestor's homeland, India, but also connects them to their country of citizenship, Fiji. On the other hand, this identification distinguishes and separates them from other Fijian groups as well as other Indian groups (Williams, 2010).

With regard to the Indian communities in New Zealand, Bandyopadhyay's (2010) important edited book addresses the post-1990 situation of the long-established Indian communities in

\footnotetext{
${ }^{3}$ In more recent work, Holmes et al. (2011) have extended the approach to identities from language choice to a range of dynamic and more discursive features, including directness, explicitness and referring to cultural values.
} 
New Zealand from a sociological perspective. The second part of the book focuses on local identities among Indian communities in different cities, e.g. Wellington, Auckland and Christchurch, addressing "[the] complexity in life histories of Indians in New Zealand", in particular "the process of construction and maintenance of a 'Kiwi-Indian' identity" (Bandyopadhyay, 2010, p. 12). Within this collection, Williams (2010) examines the problems of cultural identity that the young Indian generation in Wellington experience when growing up in two different cultural worlds. His Gujarati-majority participants identify themselves as both Indians and New Zealanders at times while they feel at other times that "neither term quite captures their identity" (Williams, 2010, p. 109). Similarly, Gilbertson (2010, p. 134) reports that the Gujaratis of Wellington depict themselves as being Indian and Kiwi (New Zealander) while some of them feel "between two worlds, belonging fully in neither". Recently, Hussain (2019) has examined negotiations of ethnic identity among more recent Indian migrants in Auckland in relation to their Kiwi identity. Hussain highlights the significance of language and religion as sources of identity, and the role of parents in encouraging the development of a hybridised identity among their children for the purpose of maintaining both ethnic and religious affiliations as well as encouraging a strong identification with New Zealand.

Joseph Edwards begins his introductory book 'Language and identity' by declaring that "[a]ny investigation of language that considers only language will be deficient, and inappropriate limitations and restrictions can cripple insights" (Edwards, 2009, p. 1). In doing so, he highlights the importance of identity, identifying linguistic research on identity as shifting the focus from a language communicative purpose to "a stronger focus upon the symbolic and 'marking' functions of language" within sociolinguistics, studies covering a plethora of HL communities have revealed various identity-related results. Many studies (e.g. Albirini, 2016; Bloch \& Hirsch, 2017; Lee, 2013; Revis, 2015; Roberts, 2005; Starks, 2006) have shown that language is central to group identity; members of the communities express the importance of language to their membership within the groups. As Smolicz notes, language is one of "the building blocks of the group's cultural identity" (Smolicz, 1997, p. 67) which delineates group membership boundaries.

In contrast, language is more typically viewed as one of numerous ethnic markers but not a central one for maintaining ethnic identity (Beswick, 2007, pp. 42-43). Such a view represents language as a secondary characteristic of ethnicity (Eastman, 1984; Edwards, 1994). Being secondary implies that if language shift takes place, ethnic identity will remain intact because "the only change being made is in what language we use" (Eastman, 1984, p. 274). That is, 
members of ethnic communities may identify as group members but do not speak the language (e.g. Dweik et al., 2019; Häberl, 2006), or do not feel it is necessary to speak the heritage language in order to be a member of the community (e.g. Holmes et al., 1993; Ngaha, 2005). Assuming and abandoning the ethnic identity, according to Beswick (2007), rely on the social situation of the group. Beswick (2007, p. 35) further posits that

when the social situation of a given ethnic group alters for the better, members may choose to maintain their identity with that group. However, if this social situation deteriorates, members may choose to redefine themselves with a different ethnic or social grouping.

Beswick here evokes Fishman's (1985, p. 60) 'rewards' that are available only to members of ethnic groups who may choose to speak the majority language. This linguistic choice, according to Eastman (1984, p. 274) is 'harmless' to identity and is justifiable because "language knowledge and use do not affect our underlying or primordial ethnic identity". If the 'linguistic capital'(Bourdieu, 1991) is not rewarding in the 'linguistic markets', the heritage linguistic capital will no longer be of value to the members of the community and consequently they will opt for the majority language. These members might look at language shift or change as "only affect[ing] one aspect of [their] ethnic identity - the language use aspect, which is a very low level manifestation of [their] cultural belief system" (Eastman, 1984, p. 274).

The relationship between HL and identity is thus controversial. Similar controversy occurs when ethnic identity is at stake. As is mentioned above, some scholars (e.g. Bloch \& Hirsch, 2017; Roberts, 2005; Smolicz, 1997; Starks, 2006) support the idea that language is a central identity marker while others take a more contentious stance by noting that language symbolically serves as a marker of group membership (Edwards, 1994, p. 146), and although language is valuable to articulate our identities, it is not the only way to do so, especially in diverse communities (Beswick, 2007, p. 225). Roberts (2005) notes that Gujarati immigrants, in the past, were not identified as members the New Zealand society, whereas the Dutch (based on their whiteness) were more likely to be identified as members of the wider society although both (the Gujaratis and the Dutch) did not speak English at that time. Similarly, the overwhelming majority of people in the 'Arab world', as the name suggests, identify by language regardless of religious or other backgrounds. In Central and South America, most people speak Spanish, but they do not identify as 'Spanish'. Hindi and Gujarati speakers follow the same faith but speak different languages. One can also notice examples where language is accompanied by national or geographic labels, e.g. 'American English' and 'Fijian Hindi'. Different theoretical positions and stances lead to different emphasis in these discussions. 
Identity and identification with one's HL may be conducive to positive or negative attitudes towards that language which in turn enhance or impede HL acquisition/learning and language choice as well. There are examples in Seals's (2017b) research where children position themselves as (non)speakers of their HLs according to how they are identified by parents and teachers. Another striking example is King's (2013) study of a transnational family discussed above. Two of the sisters are positioned differently, and this positioning in turn influences their language use where the Spanish-speaking-framed sister tends to use Spanish at home and feels inadequate speaking English, whereas the English-speaking-framed sister uses English but not Spanish.

Since identity is often indexically enacted (if one follows the social constructionist view), the following section discusses indexicality, which will be used in Chapter Five of this thesis.

\subsection{Indexicality}

Within the realm of linguistics, indexicality has been long discussed, especially in the field of semantics to describe pointing to people (I, we), time (now, today) and place (here, there). It was further applied in sociolinguistics and linguistic anthropology to clarify the relationship between language and (gender) identity (e.g. Bucholtz \& Hall, 2004; Ochs, 1992; Silverstein, 2003). The concept of indexicality originally evolved from Peirce's (1960) theory of semiotics. Peirce categorised signs as a symbol, an index and icon as per the relationship between the presentative character and what it refers to. The differences among these types of indexicality are ascribed to the way they refer to their referents. An icon is a sign similar to what is being presented (Peirce, 1960). For example, a picture of a book could be perceived as an icon that represents a book. Since an index usually refers to its referent through 'contiguity', 'causal' or some 'physical connections' (Ponzio, 2006), a book picture could also be interpreted as an index of a library. Furthermore, a picture of notebook could be a symbol of a list of contacts on cellular phones. Although the notebook has no resemblance with what being presented (list of contacts), it is connected through a social convention (Ponzio, 2006).

Within (applied) sociolinguistics indexicality refers to the social meaning (as opposed to denotational meaning) that a (linguistic) form carries in a certain context (Silverstein, 1976). Ochs (1992, p. 335) defines indexicality as "a property of speech through which cultural contexts such as social identities (e.g. gender) and social activities (e.g. a gossip session) are constituted by particular stances and acts." That is, speakers frequently use a linguistic feature as an emblem of a social identity (e.g. gender identity). Ochs (1992, p. 337) further suggests 
that the relationship between language and gender is not direct; rather, it is "mediated and constituted by the relation of language to stances, social acts, social activities, and other social constructs". That is, (continuing to focus on gender for illustrative purposes) a linguistic feature is not exclusively used by men or women. Features "may be used by/with/for both sexes [sic]" (p. 340), but knowing how the majority of speakers are thought to use a linguistic form indicates how this form can be used in the same social context to perform stance-taking, social acts and social activities (i.e. shared socio-cultural knowledge). This shared knowledge helps us understand how the speaker "does" social identities. Moreover, Ochs (1992) distinguishes between 'direct' indexicalities, which are "associations between stances [e.g. toughness] or social activities [e.g. jokes] and a particular linguistic form [e.g. swearing]" as discussed in Meyerhoff (2013, p. 267), and 'indirect' indexicalities which connect a certain stance (e.g. casual solidarity by using 'dude') with a social group (e.g. white masculinity) (See Kiesling, 2004). Indexicality, in this sense, is used to show how social forms contribute to the construction of social identity.

More recently, indexicality has expanded to include signs other than linguistic features. Yoder and Johnstone (2018, p. 196) define indexicality as "the way any meaningful sign (linguistic or otherwise) points not only to its denotational ('dictionary') meaning but also to the context or contexts in which it commonly occurs". This definition gives more room to study the indexicality of not only linguistic signs (e.g. sociolinguistic style), but also other verbal (e.g. phrase (See Yoder \& Johnstone, 2018 for the study of 'bikelanes') and non-verbal signs (e.g. colours), which are the focus of my analysis in Chapter Five.

In an attempt to organise various indexicalities, Silverstein (2003) refers to 'indexical orders' as chains of meanings that are associated with a linguistic form. These indexical orders are based upon cultural realizations (ideological or stereotypical) that associate a form with group membership, that can be extended to associate this form with a stereotypical property of this group, and then associate the same form with some other association of that property, and so on (Eckert \& McConnell-Ginet, 2013). Drawing upon and extending Silverstein's indexical orders (2003), Eckert (2008, p. 454) points out that there is a network of potential meanings, an 'indexical field', aligned with using a linguistic variable or a certain style. An indexical field is a set of potential meanings connected to the speaker/hearer's ideology that helps (re)produce social meanings. This indexical field presents these indexicalities as parts of a set of potential indexicalities. 
Eckert (2008) provides examples of using the 'hypo-hyperarticulation' of (ING) and $/ \mathrm{t} / \mathrm{release}$ to demonstrate the fluid nature of indexical fields, noting that Silverstein's first indexical order indexes "membership in a population", while second indexical order is a result of the continual interpretation of the form in context which indexes that "speakers position themselves with respect to the elements of character selected out for internal use" (p. 463). An illustration of an indexical field of /t/ release in American English included social type (e.g. school teacher, nerd girl), permanent quality (e.g. educated, elegant), and stance (e.g. polite, formal, emphatic) (p. 469). Another example is provided by Holmes et al. (2011). They show how particular linguistic and discursive features are indirectly associated with ethnicity and leadership (as components of social meaning) through stance-taking in interaction.

A semiotic/linguistic behaviour (act/habit) can also be associated with different indexicalities (Anderson, 2008), with the contextual ideologies at play. In the following speculative example, speaking Telugu (as a linguistic act) can be associated with different indexicalities whose value can be determined by the context in which the act occurs.

'Speaking Telugu' can index 'a Telugu speaker'

'someone who speaks more than one language'

'someone who is not proficient in English'

The above are some examples of the potential indexicalities of the linguistic act (speaking Telugu). While 'a Telugu speaker' embodies the direct indexicality of the action, 'someone who speaks more than one language' and 'someone who is not proficient in English' present indirect indexical meanings that could be associated with this act. The context, where the hearer's ideologies about the act gives more value to one indexicality over another, determines these indexical orders. The values given to these indirect indexicalities depend on the sociocultural context that shapes their organization and in turn the order of these indexical meanings leads to Silverstein's indexical orders (2003), i.e. 1st order, 2 nd order ... etc. That is, if a person speaks Telugu, the hearer will likely identify the speaker as a Telugu speaker (not necessarily native or otherwise). However, the act of speaking Telugu, say outside India (e.g. in the UK), may produce a more abstract level of association, i.e. indirect indexicality. As "functions performed by particular [semiotic] resources in one place can be altered in another place" (Blommaert, 2005, p. 72), this act could have a different social meanings, i.e. this person is either multilingual or not fluent in English, depending on the socio-cultural values that the hearer has. 
Indexicality in general is dynamic and changing (Ahmad, 2007), and colour indexicality (which will become relevant later in the thesis) is popular in everyday communication. We use colours to modify and/or add semiotic social meanings to our thoughts. A historical example is that pink was often referred to index (British) English in the past. Since Commonwealth countries were coloured pink on maps, pink used to be associated with English (especially British English), but younger generations seem to associate English with colours other than pink, such as yellow and blue (e.g. Salo \& Dufva, 2017; Seals, 2017b). Another exemplar of this is the usage of black and white as having opposite indexical meanings. When black is used as a modifier in terms, such as 'blacklist' and 'black market', it typically adds illegality or badness to what is being presented (list and market). Conversely, white is associated with 'good' things. Therefore, using white as a modifier in terms such as 'white lie', and 'white dove' mitigates the (negative) meaning the head noun denotes. While lying is perceived as a bad behaviour, using 'white' to modify its meaning supplants its denotation to become a "less bad" behaviour. Additionally, white can be used to add other shades of meaning to what is being described as in 'white dove', i.e. a 'white dove' indexes 'peace'. Such a change in meaning cannot be standardised because it is arbitrary and socially constructed.

\subsection{Gaps and research questions}

Based on the literature reviewed in this chapter, this study identifies and addresses the following gaps.

First, while LMLS research is a rich and developed field in New Zealand, there is a dearth of linguistic research concerning the Hindi community. The only known study in New Zealand was Jamieson's (1980) study of a group of European, Asian and Pasifika languages. Jamieson (1980) conducted a survey of non-English speaking groups in the suburb of Newtown, Wellington, focusing on the languages used and the attitudes held towards English. Jamieson identified eight major language groups (Māori, Indian, Samoan, Other Pacific Islands, Greek, Eastern European, German and other Asians) spoken in houses with preschool children. Noting that Jamieson's study included only four Hindi households, the Hindi community which has grown significantly since the 1980 s was not the focus of the project. Additionally, there have been significant methodological advancements in the field since the survey was carried out in 1980. Roberts (1999) focussed on the Gujarati community and Shameem (1995) on Fiji Hindi. Hindi as the language of India deserves its own attention. 
Second, previous identity research concerning Indian communities in the New Zealand context has mainly investigated the negotiations of ethnic, cultural and religious identities among ethnic groups (Gilbertson, 2010; Hussain, 2019), while this thesis addresses linguistic identities which have not been explored yet. Additionally, while these studies have taken hybridity as a point of departure either at community or individual levels among adult migrants (Fuchs et al., 2010; Gilbertson, 2010; Williams, 2010), this thesis looks at how young HLLs, hailing from diverse families, develop and negotiate their identities at an often-unexplored level, namely the HL school and the family. Moreover, previous research has described the depiction of cultural identity as monochrome, e.g. Indian cultural identity (Williams, 2010), while this thesis addresses cultural and national identities as multiple, e.g. multicultural identity.

Third, previous research on identity from an indexical lens has explored how adults index their socially constructed identities (Ahmad, 2007; Eckert, 2008; Hazenberg, 2017; Kiesling, 2004). This leads us to acknowledge the scarcity of indexicality research with children, especially in HL research and sociolinguistics at large.

Fourth, despite the growing interest in language practices in different domains such as the family, FLP researchers note that family structure is no longer as homogenous as previously assumed, and they call for explorations of actual rather than reported language practices (King $\&$ Fogle, 2017) that include more unconventional family configurations (Fogle, 2012).

Finally, while New Zealand is home to over 160 languages, there is little HLE research that focusses on Indian languages, especially Hindi. Researchers have so far addressed HLE for various HLs including te reo Māori, Pasifika languages and Ukrainian (e.g. May \& Hill, 2005; Seals, 2017a; Seals \& Olsen-Reeder, 2017). However, the majority of HLs that fall under the category of languages with no institutional support (like Hindi) have not been a focus. By focusing on the Indian communities who identify with Hindi in New Zealand, this thesis contributes to the New Zealand scholarship in this area. The study will be guided by the following research questions in the context of Hindi speakers in New Zealand:

RQ1: How does affiliation with the HL school connect HLLs to national/cultural identities?

RQ2: How do language practices in the HL school relate to multilingual identity development?

RQ3: How do HLLs interact with their linguistic and national/cultural identities in momentto-moment interaction? 
The following chapter elaborates on the methodology used to operationalise these research questions. 


\section{Chapter Three: Methodology}

\subsection{Introduction}

This thesis aims to explore how the realities that HLLs live connect to identity negotiation and development in social interaction. In particular, it focuses on a group of learners of Hindi as a heritage language in New Zealand - a group that is under-explored as outlined in the previous chapter. To achieve this objective, an ethnographic approach is adopted as it allows for a holistic picture of the dynamic configuration of the school communities and deep insights into the interactional complexities within the school. This way, this thesis aligns with the recent calls for shifting from traditional methods in LM and HL research (which mostly rely on the macro-level analyses and reporting data) to the micro-level of interactional analysis (e.g. Holmes, 1997; Pedraza, 2015; Schwartz \& Verschik, 2013). As shown in Chapter Two, traditional methods of data collection in LMLS (and of course HL) research embrace collecting respondents' reported language proficiency, use and attitudes by surveys and interviews, (e.g. Al-Sahafi \& Barkhuizen, 2006; Boyce, 1992; Jean Kim, 2019) or by means of language performance tests (e.g. Gharibi, 2016; Shameem, 1995). In contrast, the current line of research adds ethnography (e.g. Revis, 2015; Yilmaz, 2016), recordings of naturally occurring interaction (e.g. Gharibi \& Seals, 2019; Revis, 2017; Yu, 2010) and drawings (e.g. De Bres, 2017; Purkarthofer \& De Korne, 2019; Seals, 2017b; Solano-Campos, 2015) to their toolkits. These trends in LMLS research allow for the navigation between the macro level of analysis (e.g. language policy and planning) and micro levels where participants' linguistic realities are reflected discursively and where participants (as social actors) enact, perform, negotiate and develop their identities in social interactions. In general, I decided to use an ethnographic approach because it is an 'interpretive approach' (Copland \& Creese, 2015) that provides researchers with details to explain and give depth to the linguistic analysis (Creese, 2010, p. 141) by scrutinizing language within its cultural context.

Additionally, as a researcher who wished to be a friend of the community, adopting an ethnographic approach allowed me to build rapport with my participants and gain better access to the community. Also, my participants included children and some of refugee background, so the ethnographic approach was helpful for working with these vulnerable groups since it enabled me to build trusting relationships with participants to explore how their interpretations of the world are shaped (Best, 2013; Bogdan \& Biklen, 2007; O'Leary, 2017) as well as to gain a genuine understanding of what interactions mean to them (Eckert, 2000). 
In this chapter, I elaborate on the purpose and design of the methodology used in this thesis, including a discussion of some of the central methodological issues encountered during the data collection and analysis processes. I first begin with the rationale behind choosing the ethnographic approach. I then discuss the research design, providing a detailed description of the data collection process, my access to the community, the participants as well as data analysis. The ethical considerations are presented towards the end of the chapter.

\subsection{Why an ethnographic approach?}

As this thesis is targeted at working with a community language group, the methodological choice needs to match this goal. The ethnographic approach is useful for working on dynamic social constructs (Heller, 2008) within small groups such as HL communities (Best, 2013). Because social constructs such as multilingualism and identities do not exist in a vacuum and instead emerge in social and cultural practices, ethnography offers more in-depth access to the complexities, contradictions, and meanings of social actions when described and interpreted in natural settings (Best, 2013; Heller, 2008; Riazi, 2016).

Ethnography finds its roots in cultural and linguistic anthropology and some branches in sociology (Paltridge \& Phakiti, 2015) as a means of studying "ethnic or cultural groups living in either their natural social settings or in alien communities" (Riazi, 2016, p. 108). Ethnographers adopt an emic perspective to capture participants' views of their own social actions and the meanings of these actions (Heller, 2008; Riazi, 2016). Ethnographic approaches have been extensively used in applied linguistics research after the 'social turn' in language study which engendered the need for developing in-depth understandings of language learning and teaching events in the social contexts within which they take place (Starfield, 2015, p. 139). Ethnographies offer knowledge that can be difficult for participants to articulate or that can be erased easily in reports of the participants' lived experiences (Rampton et al., 2004, p. 7). The new intellectual climate of late modernity (e.g. globalisation and superdiversity) and post-structuralism led to the development of a new interdisciplinary approach that UK scholars (e.g. Rampton, 2007; Rampton et al., 2004; Tusting \& Maybin, 2007) have developed and called 'Linguistic Ethnography', which I adopted in this thesis.

Copland and Creese (2015, p. 13) define Linguistic Ethnography (LE) as "an interpretive approach which studies the local and immediate actions of actors from their point of view and considers how these interactions are embedded in wider social contexts and structures". Copland and Creese emphasise the centrality of language and linguistic signs to LE, especially naturally occurring interactions and communicative practices shaped by social actions and 
realities (Kulavuz-Onal, 2018, p. 118). To achieve this, linguistic ethnographers seek to collect interactional data from various sources, including in-depth interviews, observations, written texts and documents, and additional interactional data (Copland \& Creese, 2015). As discussed in the data collection part of this chapter (Section 3.3.3), I prioritised interactional data from recordings of home interactions and my observations in the HS, which were supplemented and enhanced by fieldnotes. I also used semi-structured interviews and linguistic reflection drawings and their verbal descriptions as interactional encounters rather than sources of reported data (Talmy, 2010, 2011), as outlined in Section 3.3.5. Capturing this interactional data enabled me to engage in the moment-to-moment of the recorded activities to follow steps the participants took to make meaning and establish common understanding (Pérez-Milans, 2015). This was enhanced by using detailed transcriptions of the interactional data and finegrained methods of data analysis, such as discourse analysis in the form of interactional sociolinguistics. As discussed in the data analysis section of this chapter, my analysis of the data aligned with the analytical disposition that linguistic ethnographers share by "focus[ing] on data and close analysis of situated language in use" (Copland \& Creese, 2015, p. 29). I also fully transcribed the data, looked for patterns to code, and used standard interactional sociolinguistic techniques (Gumperz, 1982) to analyse the recordings in context. In line with this approach, I recognised the researcher's voice and positionality, reflexivity, and ethics at all stages of the research.

As mentioned earlier, unlike traditional ethnography, LE takes language as its point of departure. It also focuses on topics such as professional interaction, literacy events, and speech style rather than comprehensive descriptions of speech communities (Rampton, 2007, p. 592). Thus, LE provides useful insights into group practices in a particular social activity (e.g. HL learning) rather than everyday life. These characteristics of LE, i.e. its suitability for literacy events as well as its 'topic-oriented' nature rather than comprehensive description of speech communities, provide a good fit with the goals of this research. In order to conduct a close analysis of interactional data required by linguistic ethnography, I carried out an in-depth investigation of meaning-making practices in the HS (i.e. a space for literacy events) and three families connected to it as a case studies to focus on identity work (i.e. topic) (Blommaert \& Jie, 2010).

Another point to highlight from the definition above is that LE does not gloss over macro issues and 'cultural ecologies' (Rampton et al., 2004, p. 2). Instead, the synergy between ethnography and linguistics "links the micro to the macro, the small to the large, the varied to the routine, the individual to the social, the creative to the constraining, and the historical to the present 
and to the future" (Copland \& Creese, 2015, p. 26). That is, LE lends itself to studying the meaning and significance of a form or practice in combination with the socio-cultural organisation/process (Rampton, Maybin, \& Roberts, 2015, p. 15). Thus, LE has brought together ethnography and linguistics (notably critical discourse analysis, conversation analysis, interactional sociolinguistics and the new literacy studies) as an interpretive approach to analysing the participants' practices embedded within a wider social context (Creese \& Copland, 2017; Riazi, 2016; Starfield, 2015).

Additionally, LE conceptualises the contact between the researcher and the researched as cooperative in nature, flattening the hierarchies, and facilitating access to participants' information (Norton \& De Costa, 2017; Sercombe, Garner, \& Raschka, 2006). LE, therefore, constructs the participants as coresearchers and emphasises researching "with" rather than "on" participants (Creese \& Copland, 2017, p. 349). Yet, due to LE research traditions where researchers work with close-at-hand groups, it is also suggested that the researcher should "get analytic distance" (Rampton, 2007, p. 590) to accomplish what is known in traditional ethnography as making the familiar practices 'strange' (Atkinson, 2004).

Pérez-Milans' (2015) chapter on language and identity in LE discusses the influence of the conditions of late modernity, such as challenging fixed ideas about culture, language and identity, which have led to "growing complexity and unpredictability of the way social life is arranged through daily practices" (p. 87). Pérez-Milans elaborates that these conditions encourage an ideological shift from the fixed understanding of language as bound to a certain nation to a more dynamic view where multilingualism is seen as a societal privilege. This sits alongside researchers' analytic shifts towards trans-local, trans-cultural and trans-lingual practices (Pérez-Milans, 2015, pp. 86-87). In line with its aim "to use discourse analytic tools in creative ways to extend our understanding of the role language plays in social life" (Creese, 2008, p. 235), researchers tend to work in a wide range of areas including transnational (e.g. Cogo, 2016; Soler \& Zabrodskaja, 2017), HL (e.g. Creese, Blackledge, \& Takhi, 2014; Revis, 2015), and identity research (Blackledge \& Creese, 2017; Creese \& Copland, 2017; Norton \& De Costa, 2017; Pérez-Milans, 2015).

LE is thus well aligned with the goal of this thesis, i.e. an examination of how heritage language learners of Hindi negotiate and develop their multilingual identities in heterogeneous contexts: it views the use of linguistic patterns as social experiences and offers "close analysis of situated language use [that] can provide both fundamental and distinctive insights into the mechanisms 
and dynamics of social and cultural production in everyday activity" (Rampton et al., 2004, p. 2).

\subsection{Research design}

\subsubsection{Gaining access to the community}

As a new migrant to New Zealand at the start of my PhD, I was not initially aware of the dynamics of the local Indian communities. It was one of the inevitable challenges that I encountered at the beginning of the research journey. Levon (2014) and Meyerhoff, Schleef, and MacKenzie (2015) suggest that outsider researchers should contact community members through a friend or acquaintance, i.e. friend-of-a-friend technique (Milroy, 1980, 1987), and through official community brokers or gate-keepers, e.g. teachers, leaders and associations. Being an outsider researcher has traditionally been seen as a deficit in research, and researchers have tended to involve co-researchers who belong to the group being studied to temper this issue (e.g. Holmes et al., 1993; Umali \& Bell, 2017). However, being an outsider was also rewarding as discussed in the following section. As a practitioner in the field, I recognised my status as an outsider and reflected upon the outsider and insider perspectives alike. Conducting this research reflexively led me to recognize different levels of interpretation of the dynamics within the community that might not be noticeable to insiders alone (this is discussed in Section 3.3.2).

Two ways of acquainting myself with these communities were particularly helpful. First, as an insider of the Muslim community, I had the chance to make friends of Indian descent through the mosque, which enabled me to initially explore the wider Indian community and communities within it. Second, I also had the chance to be connected with gate-keepers, namely the president of Community Languages Association of New Zealand (CLANZ) and the operations manager of a local Hindi School. These connections enabled me to pay visits to the Hindi School sites as an attempt to increase access to the 'social capital'4 (Bourdieu, 1986), which I needed for data collection. In so doing, it was easier for me to meet a wide range of members of the communities connected to the school.

Several ways of contacting community members have been used in New Zealand. Previous researchers have talked to or emailed members of the community, or joined clubs, groups and

\footnotetext{
4 Putnam (1995) defines social capital as "features of social organisation such as networks, norms and trust that facilitate coordination and cooperation for mutual benefit" (p. 66).
} 
places where people gather (e.g. Hay, 1994; Revis, 2015). Others included co-researchers who belonged to the community to add insider-like status to their research (e.g. Roberts, 1999). For the current research, I adopted relatively similar ways to approach potential participants. I approached my participants through a third party (Milroy, 1987), e.g. teachers and community key figures, and by talking to them in the places where they gather, e.g. visiting the school (by invitation) during the end of the year ceremony. Thus, I was introduced to the participants by highly respected community members. This method effectively allowed me "to enter the community as something other than stranger" (Levon, 2014, p. 201), and helped members of the community feel more comfortable and so "they [felt] encouraged to participate in the research and help[ed] the researcher establish a more personal connection" (Levon, 2014, p. 201).

\subsubsection{Constructing my role as an ethnographer}

At this point, it is useful to to discuss the notion of reflexivity prior to giving an account of my role as an ethnographer. Reflexivity is an integral part of the research process (Heller, Pietikäinen, \& Pujolar, 2018; Hennink, Hutter, \& Bailey, 2020; McKinley \& Rose, 2020; Schwandt, 2007). My involvement in the social action throughout the research brings reflexivity to the fore. Reflexivity refers to the process of "critical self-reflection on one's biases, theoretical predispositions, preferences, and so forth" (Schwandt, 2007, p. 260). Schwandt further describes reflexivity as an acknowledgment in which "the inquirer is part of the setting, context, and social phenomenon he or she seeks to understand" and as a means that enables the researcher to critically inspect the entire research process (Schwandt, 2007, p. 260). Being an integral part of the entire research, reflexivity involves the interaction between the researcher and participants, research settings and procedures (Glesne, 2011). This includes acknowledging the researcher's background, position and emotions (Hennink et al., 2020). Reflexivity encompasses acknowledging subjectivity and positionality. Subjectivity concerns the researcher's epistemological stance and the intervention of their personal experience, background and opinions in the topic being researched and in interpreting the data (McKinley, 2020). In ethnographc research, subjectivity is addressed by establishing transparency. In this regard, Wei (2020, p. 161) noted that the need to include a detailed and honest account of the researcher's influence on the research including their trajectory, stance, motivation and skills. By contrast, positionality refers to how the researcher identifies themselves in the social action (McKinley, 2020). McKinley (2020, p. 5) discussed four research positions a researcher might identify with, namely, insider (sharing a cultural background with participants and/or research 
site), outsider (having no cultural experience), in-betweener (having partial shared experience), and halfie (having mixed national or cultural identity due to migration, overseas education, or parentage). In this reseach my positionality was constructed as closest to an 'in-betweener'. As demonstrated below, my participants and I recognised my position through the cultural/national/linguistic aspect activited in the interaction.

In summary, reflexivity means that the reseacher "understands that he [sic] is part of the social word(s) that he or she investigates" (Berg, 2007, p. 178) and requires us to engage in "an ongoing conversation with yourself" (Berg, 2009, p. 198) in order to ensure legitimate and valid research process (Hennink et al., 2020). In the same vein, Heller et al. (2018) discuss the researcher's positionality and power relations in research, noting the importance of thinking about the sensitivity of the researcher's questions and considering the way the researchers ask questions. Thus, I critically questionned my position, power relations, my decisions and interpretations on an ongoing basis in order to minimise any subjective perspective, fixed understanding or assumptions.

This research involved interaction between me and the community I worked with; my participants and I co-constructed my role through the different stages of this research. This role generally depended on the context where I was alternately positioned as an insider or outsider. Blommaert \& Jie (2010, p. 33) reflect on the researcher's status, noting that researchers "often travel from an innocent outsider to a knowledgeable member of the field, and [they] therefore need a careful record of that trajectory." While this quotation highlights the extremities of the researcher's positionality in the research, it foregrounds the significance of reflexivity while moving in that trajectory. In this research I have been positioned differently on various occasions. I was positioned as an outsider, especially in the early stage of data collection. For example, the teachers presented me as a "PhD student" and "researcher", and referred to me as "from Jordan". Three weeks later, while I was at site C, Sun ji commented on one of the students' reading, saying:

I wish Mohammed could understand that as well + so + can you just repeat what you said in English + so that Mohammed knows what you guys are doing + right + Havana + here is an opportunity to speak English know [laugh] which is much easier.

Notably, Sun ji was positioning me as an outsider who does not speak Hindi while simultaneously aligning me with the students who were also positioned the same way. I interpreted this as Sun ji wanting the student, Havana, to render the essay in English to get me included in the activity as well as giving her some kind of comfort when using English. Thus, 
Sun ji positioned me and the students alike as speakers of English. My status became more complex as the positioning became dynamic and multi-faceted. Sometimes, I was purposefully positioned as an outsider. As will be discussed in Chapter Four (Section 4.4.2), Savita ji, a teacher at site B, benefitted from my presence to make the students aware that their behaviour in class would be considered unacceptable by others.

Not only did the teachers sometimes position me as an outsider, but the students did as well. An exemplar of this is demonstrated in Section 4.4.3 when the students greeted one another (and their teachers) by saying 'namaste'. Yet, they greeted me and their parents differently (saying, e.g. 'hi' and 'good morning'). In so doing, they positioned me and their parents alike, as outsiders of their HS group.

Notwithstanding this, at other times I was positioned as an insider. This included being a community language speaker, father and multilingual person. Being a migrant was often activated in my discussions with the teachers and parents about the efforts exerted to gain institutional support for learning Hindi in New Zealand. Mostly, these discussion included emotional aspects of linguistic identities in which my interlocutors paired Arabic with Hindi (and other languages) to index similarities in terms of language rights.

As noted in Chapter Four, multilingualism is celebrated in the HS. The teachers encourage the students to be proud of their languages while using me as an example. A teacher once introduced a Hindi poem about languages and commented on the students' multilinguistic abilities. Then she referred to me saying "Mohammed speaks three languages, just like you guys". This way, the teacher positioned me and the students alike as belonging to one group when talking to the children about multilingualism. Additionally, my status as an insider was also constructed by parents since I am a father who was expected to have the same struggle with his children. This positioning was often connected to evoking the hypothetical example of "how would you get your children to learn Arabic in New Zealand?"

The dynamic and multilayered construction of my status according to activated membership at different points in time transformed this research into a 'joint activity' (Bucholtz \& Hall, 2005) in which my status plays a key role in the quality and nature of the data obtained (Hazen, 2000; Lichtman, 2013).

The outsider status provided me with the opportunity to benefit from my 'innocence' (Blommaert \& Jie, 2010) because of which I was able to inquire about cultural elements that I could not figure out easily without losing face (Wilson, 2011). In addition to that, my participants provided me with detailed explanations and justifications of their practices in the HS as they knew I was not privy to the shared understanding to interpret their interactions. The 
outsider status also enabled me to capture practices hidden or invisible to insiders as they may take them for granted as normal practices (Salahshoor, 2017).

Schilling (2013) points to the value of conducting sociolinguistic research from a dual insideroutsider perspective. Thus, my involvement with the community to empower my insider status required devoting time and efforts to building a strong rapport with my participants. This included attending and participating in events organised by the school after the observation period at which I assisted the school in organisation and set-up. Despite the fact that I did my best to foreground my role as a learner (Schilling, 2013), community members positioned me as an expert who could advise them in, for example, how to encourage their children to speak Hindi at home. On other occasions I was asked to contribute an article on the benefits of multilingualism to the 2017 issue of the school journal 'Darpan' (Appendix A).

As suggested by Eckert (2000) in her work with the students at Belten High in Detroit, I regularly aligned with the students in the HS rather than teachers by spending the break time chatting and playing with them. This way, the feeling of being insider every now and then was supportive of my efforts to build rapport with my participants which in turn resulted in their increased willingness and comfort in sharing their experienes and beliefs with me (Jean Kim, 2019).

\subsubsection{Data collection}

Within the realm of linguistic research two paradigms of research are frequently used, namely, cross-sectional studies and longitudinal studies. While researchers in cross-sectional studies collect data at one point in time (synchronically), longitudinal studies encompass collecting data over extended and multiple periods. One of the merits of longitudinal studies is that they enable us to observe change over time, but there are challenges related to them, e.g. sample retention and resources (Rasinger, 2010). This study included aspects of the two paradigms in the sense that I was involved with the community longitudinally, but the data itself was synchronic from different sources within the community, drawing upon qualitative methods to get a picture of the complexity of the community at hand and deeper insights into their linguistic behaviour as undertaken and experienced by people (Heller et al., 2018). The research design used in this study encompassed an ethnographic approach in the sense that it includes but is not limited to ethnographic interviews accompanied by observation. Data was collected in three phases so as to enrich data interpretation. The phases of data collection are illustrated in Figure 3.1, with a special focus the on school in phase 1, community members in phase 2 , and the home in phase 3 . 


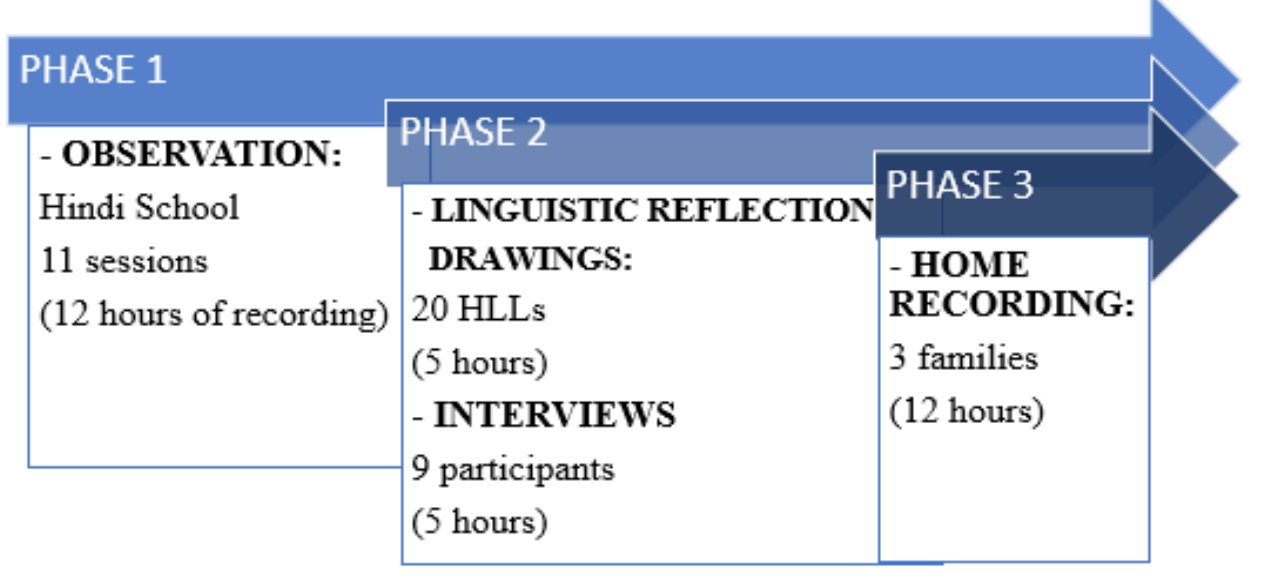

Figure 3.1 Phases of data collection

The first phase narrowed the focus of the study and gathered data pertaining to multilingual practices at school, seeking to uncover how these practices might connect the students to HL identities (RQ1). At this level, data was collected by means of limited-participant observation at a local Hindi community language school and at different Indian events. In some cases, active participation was requested by the participants. These observations also helped me to decide on the interview schedule held with community members. To alleviate the influence of being a limited-participant observer on data collection and interpretations, multiple sources of data were used, i.e. methodological triangulation. Observations took place in the HS and in different Indian events such as Diwali festival and Hindi Divas. Observing actual linguistic practices is more reliable than getting the participants to report their practices and/or language use in surveys (Holmes, 1997; Rampton, 2007). The observation at the school lasted eight weeks, i.e. the school term. It began on $5^{\text {th }}$ of August, 2017 and ended on the $24^{\text {th }}$ of September, 2017. Although I visited the school during the first week of the school term (29-30 July, 2017), I did not use any data of that visit in the analysis due to ethical considerations, namely not having the participants' consent yet. Eleven observation sessions in the Hindi classes were conducted for approximately four hours weekly, with a total of 20 hours out of which 12 hours were audio recorded. For observations and interviews, the participants preferred audiorecording and showed discomfort at the idea of being filmed. The classes were held on a weekly basis. Whereas sites A and B held the language classes on Sundays for two hours each, site C held its classes on Saturdays for one hour and a half. Consequently, I had to schedule my visits to the three sites, especially sites A and B classes, as shown in Table 3.1. At first it was puzzling to observe and record as the students were normally sitting in two groups, so I placed two recording devices on the tables to record as much as I could of their interaction. In other cases, 
i.e. in site $\mathrm{B}$, there were three classes to observe. I was fortunate that these classes were put together into one group to prepare for some upcoming events.

Table 3.1 Observations schedule

\begin{tabular}{|c|c|c|c|}
\hline Week & Site C & Site A & Site B \\
\hline 1 & 5-Aug & 6-Aug* & $X$ \\
\hline 2 & 12-Aug & 13-Aug & $X$ \\
\hline 3 & $\mathrm{X}$ & 20-Aug & $\mathrm{X}$ \\
\hline 4 & 26-Aug & $\mathrm{X}$ & 27-Aug \\
\hline 5 & $X$ & $X$ & 3-Sep \\
\hline 6 & $X$ & 10-Sep & $\bar{X}$ \\
\hline 7 (Hindi Divas) & \multicolumn{3}{|c|}{ 16-Sep } \\
\hline 8 & 23-Sep & $X$ & 24-Sep \\
\hline Diwali Festival & \multicolumn{3}{|c|}{23 -Oct } \\
\hline
\end{tabular}

Additionally, I observed two Indian events in which the school took part. The Hindi Divas, held in September, was organized by the school and took place in a local Indian center, and the Diwali festival, in October, which was nationally organized. Twelve hours of observation were documented in the form of fieldnotes, noting that the focus was only on the parents, children and teachers who had consented to participate in the study.

The second phase aimed at exploring how the language practices in the HS contributed to the HLLs' multilingual identity development (RQ2). This phase encompassed two data collection methods, namely, linguistic reflection drawings and semi-structured interviews. While the former focused on the children, the latter targeted members of the community around the school.

First, I interviewed 20 Hindi learners for the purpose of undertaking linguistic reflection drawings (Krumm, 2001; Seals, 2017b) with a special focus on delving into the participants' language ideologies towards the languages they speak as well as how they discursively negotiate their identities. Linguistic reflection drawings are also beneficial to investigate the conceptualisations of the participants' linguistic repertoire. The linguistic reflection drawing is a modified version of Krumm and Jenkins's (2001) language portraits which Krumm used in a psychological study of the connection between home language and cultural identity (as discussed in Seals, 2017b). In this task, the children are often asked to do a colouring activity accompanied by explicit metacognitive accounts of their colour choice. This is a 'think aloud' activity (Deschambault, 2017), and is one of the introspective methods implemented in applied linguistic research in which the respondents are asked to speak out their thoughts to explore 
their cognitive process during task completion (Sasaki, 2013). One of the merits of this activity is that it suits the age group of participants, children, who often express themselves more clearly when dealing with tangible things (Seals, 2017b).

The linguistic reflection drawing activity was conducted in the form of interviews right after the observation in September and October 2017. The motivation of having an interview-like colouring task was to encourage the children to explicitly spell out their thoughts through interaction. Twenty Hindi learners (13 boys and 7 girls) from the three school sites participated in the activity. Their age range was between 5 and 17 years and all were returning students. The data obtained from this activity was audio-recorded (around 5 hours), and artefacts from the children were collected (i.e. the drawings). The participants did the task individually at their respective school sites. I did my best, of course with help from teachers, to separate the children who did the activity from those who did not in order to ensure that the participants were giving genuine responses rather than copying each other's responses. I also made it clear that the questions I was asking (e.g. how many languages do you know? And why did you choose green for English?) had no correct answer and for every participant the correct answer was what they personally felt about their languages.

Before colouring, the children were asked about the languages they knew. They then chose one colour for each of their languages. Their choices were followed by questions to let them provide an account of the reasons why they chose the colours, allowing each child to offer different conceptualizations and explanations for their choice. For instance, one child chose orange to index Hindi since the colour orange, according to her, was part of the national flag of India. The children were given an outline of a human and told that it represented them. They coloured in the silhouettes with the colours they previously selected. Having finished the colouring, they were encouraged to talk about how they ordered the colours in the drawing. This enabled me to uncover the different connections with language as well as how they perceived themselves in this regard.

In addition to the linguistic reflection drawings, I conducted semi-structured interviews with nine stakeholders in the community who were linked to the school (e.g. teachers, teacher aides and parents). The interviews were audio-recorded and averaged 40 minutes in length with a total of 6 hours of recordings. The interviews were held during an informal meeting with each participant. The participants decided the time and venue of the interview, e.g. weekdays or weekends, at the HS sites, or on Victoria University of Wellington Kelburn campus. The interviews elicited the participants' demographics, linguistic background, language use, 
attitudes towards language maintenance efforts, identification, and beliefs about the future of Hindi in New Zealand (See Appendix B). When interviewing teachers, there were additional questions concerning curriculum, school language policy, the HL teachers, planning, and the learners' experiences and expectations about the Hindi School (See Appendix C). Interviews were held between October 1, 2017, and December 30, 2017.

Interviews with parents, teachers and teacher aides, and with children (i.e. the children's descriptions of their drawings) were treated as a social practice (Talmy, 2010, 2011). That is, my participants and I co-constructed the interaction and knowledge, and negotiated meanings locally among us. In so doing, I aligned with Talmy's (2010, p. 132) suggestion that researcher reflexively recognises that data is collaboratively produced rather than merely reported. Viewing interviews as a social practice enabled me to focus not only on the content but also on how it was shared. Consequently, although I had prepared interview guides, aiming to equalise power relations as much as possible during the interaction influenced the flow of the points raised to accommodate the participants. This allowed for departing at times from the interview guides as well as giving more room to the participants to ask questions to which I responded. Likewise, I analysed the interactional moves the participants made to build and negotiate meaning, and to talk about and perform identities. Another merit of dealing with interviews as a social practice is the mitigation of researcher bias due to the "reflexive orientation to the collaborative character of knowledge production and data generation" (Talmy, 2010, p. 140).

The third phase of data collection aimed at obtaining naturally-occurring home interactions to see how the HLLs interact with and respond to identities assigned to them, i.e. how identities are negotiated in every day conversations at home, and how HLLs negotiate their identities (RQ3). Home recordings give richer in-depth insights of actual language practices despite the scholarly concern of the data gained from recordings being 'tape-affected speech' (Gerwinski \& Linz, 2018). A body of research has noted that participants use the recorder as a tool to accomplish their identity work in interaction (Copland \& Creese, 2015; Gordon, 2012). Noting that the recorder is often perceived as a source of Labov's $(1966,1972)$ 'observer's paradox', and which researchers have traditionally aimed to alleviate (Cameron, Frazer, Harvey, Rampton, \& Richardson, 2018), there has been a shift in this view in more recent scholarship. For example, Heller et al. (2018) argue that the researcher's position should be recognised as a component of interaction instead of being perceived as problematic, adding that the data that comes from the participants is the product of involvement of both the participant and the 
researcher. The recordings certainly had an influence in my study, especially in Kevin's family. Kevin appeared in the recordings to have a dialogic orientation to the recorder. As will be demonstrated in Chapter Six, he used the recorder to encourage his children to show their Hindi and Mandarin literacy skills.

Home recording is arguably more reliable than the reported data in surveys and interviews where participants report what they believe or desire to tell. Three families participated in this phase after expressing willingness to audio record their children when engaging in daily conversations. I gave two recorders to two families, and the third family preferred to record using the father's smartphone. I asked the parents to turn the recorder on at the dinner table (Fogle \& King, 2013; Sierra, 2018) and during playtime (Gordon, 2009; Revis, 2015) to encourage recording potential conversations among different family members, including (grand)parent-child and child-child interaction. A total of 8 hours of recordings were collected in this phase. The families participating in phase three included a family with two Indian parents, a family with one Indian parent, and a family with non-Indian parents who had adopted an Indian girl, as elaborated in section 3.3.4. The informal conversations I had with the children and parents made it easier for me to recognize their voices in the recorded encounters. Participants were asked to listen to the recordings and delete any material they did not feel comfortable having included. Post-recording feedback from the parents was ongoing during the analysis to provide 'warrants' for the interpretation of the meanings being negotiated (Marra \& Lazzaro-Salazar, 2018), and to double-check Hindi data when the Hindi transcriber needed. Data collection in this phase took place between February 1, 2018 and June 1, 2018.

\subsubsection{Participants}

Participants were primarily selected upon availability at the Hindi School. Speakers of all languages who attend the school were invited to participate to capture the multilingual nature of the community. The school members and their families were speakers of multiple languages, including but not limited to English and Indian HLs such as Hindi, Fijian Hindi, Telugu, Kannada, Malayalam, Gujarati, and Tamil. In addition to Indian languages, some participants had access to other languages at home (e.g. Mandarin and Cantonese). The characteristics of the participants allowed for exploring the richness found within this setting and lent itself to further exploring what practices multilingual children shared, as well as their identity negotiations in multilingual contexts.

All children participants were either New Zealand-born or their families came to New Zealand when they were under two years. The children's age range at the time of the study was between 
4 and 17 years old, and all spoke English fluently. As for the parents, only one parent was New Zealand-born, whereas the rest immigrated to New Zealand as adults. At the family level, the families were characterised by different linguistic, religious, ethnic, and social backgrounds. As shown in Chapter Four, these included Hindi speaking families where both parents speak Hindi, other Indian language(s)-speaking families, multicultural families where parents spoke different languages (e.g. a Hindi speaking father and a Mandarin-speaking mother), and an adoptive family in which neither of the parents spoke Hindi, but for whom it was important to send their adopted Hindi-speaking child to the HS.

The maximum number of participants for the three phases of data collection was set at 50 people. Around 35 Hindi learners, 7 teachers/teacher aides and 5 parents participated in the first phase of data collection. The second phase originally encompassed 35 Hindi learners from those who participated in the first phase and nine adult interviewees. However, the number of children participating in this phase dropped to 20 since some parents opted out of the next stage of the research, and some families had inconsistent attendance. For the interviews, the nine participants were primarily teachers and parents who were in regular connection with the school. For the third phase, the focus was on naturally occurring home interaction in three families, with a total of 12 participants, participated. The following is a description of each of these families.

Family one (Muromaha's family, henceforth) was quite a unique family. It was comprised of two English speaking parents of European descent, Mark and Sara, and an Indian adopted daughter, Muromaha. Muromaha was born and raised in India. She came to New Zealand at the age of 7 after she had been adopted in 2017. At the time of the research, she had been in New Zealand for five months. Muromaha was a native speaker of Hindi and was learning English. Her mother reported that they registered Muromaha in the HS to keep her connected with her heritage language and culture. Mark and Sara moved to New Zealand some ten years ago and were white-collar workers.

Family two (Kevin's family, henceforth) consisted of a Fiji-Indian father, Kevin, a Chinese mother, Grace, one daughter (Havana) and two sons (Austin and Max). At the time of data collection, Havana was 17, Austin was 15 and Max was 11. The children's first language was English, but they were also learners of Hindi, Mandarin and Cantonese. This family was committed to multiculturalism, and although both parents worked full-time jobs, they attended the Hindi classes with the children, with the mother taking notes of what the children were learning at school site $\mathrm{C}$. 
Family three (Kaya ji's family, henceforth) comprised of two Indian parents, Peter and Kaya ji, and two daughters, Ann and Mylu. Ann was nine years old and Mylu was six years old at the time of the research. Peter was a New Zealand-born Indian who natively spoke English and his heritage language, Gujarati. Kaya ji was an Indo-Fijian whose family immigrated to New Zealand in her teenage years. She spoke Fijian-Hindi, Shudh Hindi and English. They both worked in the finance industry and Kaya ji volunteered at the HS as a teacher aide at site C. The daughters had attended the HS for one year. The daughters' first language was English, and they were learning Hindi and Gujarati. They were closely connected to their extended family and the girls spent a couple of hours after school with their grandparents each day.

\subsubsection{Data analysis}

This section elaborates on data transcription and analysis. I began transcribing the recorded data once each recording was done. In this way, data collection and analysis were parallel and ongoing.

Olympus Digital Voice Recorders were used to record the observation sessions, interviews and home interactions. One of the families preferred to do some recordings using the parent's smartphone. The transcription step required decisions on "how much to transcribe and what details to include" (Révész, 2012). I made these decisions based on my research questions and the analysis approach (i.e. Interactional sociolinguistics), which typically includes detailed transcription such as hesitations, pauses and overlaps to ensure ease of reading and that the meaning is fully conveyed. Some minimal editions/deletions were also made, for instance, when private issues or unconsented data came up in the interaction. The transcription conventions of all recorded data were adapted from Holmes et al. (2011) and examples were edited to protect the anonymity of the participants (See Appendix D). For each recorded file, I first listened to have a wider picture of its content. Then I transcribed the recordings using an Olympus AS-2400 Transcription Kit which included a footswitch, stereo headset and DSS standard software. The transcription Kit allowed for more accurate and easier transcription.

The data were analysed using the qualitative data analysis software NVivo12 Plus. All data, i.e. fieldnotes, interviews, linguistic reflection drawings and home recordings, were imported to the software. They were then reviewed and coded in the relevant nodes as explained below. One of the merits of using NVivo is that it operates from the Grounded Theory Approach (Bazeley \& Jackson, 2013), allowing for emergent category coding of data. NVivo enabled me to identify covered layers of meaning in the data. This characteristic was of great value to my analyses because it offered in-depth exploration of the relationships embedded in the data. 
Having transcribed the data, coding was the next step. Coding is defined as "the analytical process of organizing raw data into themes that assist in interpreting the data" (Baralt, 2012, p. 222). Coding can fall into various types. Generally speaking, Howitt and Cramer (2007, p. 282) suggest three types of coding based on data collection methods and outcomes, i.e. pre-coding, researcher-imposed coding, and qualitative coding - coding emerging from the data. While pre-coding is used in quantitative research, researcher-imposed coding enables researchers to quantify their qualitative data. By contrast, qualitative coding allows for developing "coding categories on the basis of an intimate and detailed knowledge of the [qualitative] data" (Howitt $\&$ Cramer, 2007, p. 288). The last type was used in this thesis. Throughout the analysis, codes were generated deductively and inductively (Fereday \& Muir-Cochrane, 2006). This approach allows for looking for pre-determined categories in the data that correspond to, for example, the research questions, but also allows the researcher to develop new themes evolving from their close-eye examining of the data (Fereday \& Muir-Cochrane, 2006, p. 83). For example, in line with the research questions, I had some ideas in mind while going through the data, including language practices, and indexicalities. Complementing these, themes such as 'gender talk', 'language play', and 'translanguaging' emerged in the data through open coding (Holton, 2007) or "breaking data apart and delineating concepts to stand for blocks of raw data" (Corbin $\&$ Strauss, 2008, p. 195). The coding process is an iterative process and encompassed several procedures. I started with scanning and reading through the data to look for codes that were related to my research questions as mentioned earlier. This way, I created nodes for the concepts and ideas I found in the first iteration. Next, I formulated ideas for codes during the second iteration by open coding, which was done electronically using NVivo (Baralt, 2012). I then did further detailed iterations, coding the data for one theme at a time to make sure that my coding was consistent throughout. Furthermore, using analysis software like NVivo helps enhance the credibility of the coding as it keeps historical archives of the project and presents links visually (Baralt, 2012, p. 228).

The provision of rich context allows for a better understanding of the linguistic practices that individuals have (Blommaert \& Jie, 2010). Thus, the concept of 'Communities of Practice' (Lave \& Wenger, 1991; Wenger, 1998b) helped scrutinize these practices in as 'situated' way and will be used in my analysis as presented in Chapter Four. 


\subsection{Ethical consideration}

Prior to starting fieldwork, I applied for ethics approval from the Human Ethics Committee of Victoria University of Wellington. Within this process I took efforts to design information sheets, adult consent forms and child assent forms for all data collection methods, as well as filling out the ethical approval application. This helped me to reflect upon fieldwork, especially the practical issues concerning anonymity and confidentiality of my participants' details. The information sheets, consent and assent forms were written in plain English to make clear the purpose of the study. They also highlighted what kind of data would be gathered and why. These forms concluded with a clear statement that participants held the right to withdraw from the study whenever they wished before December 1, 2018, which would mean that their data would not be used in the research. The participants also reserved the right to receive a copy of their (children's) recording (if recorded), read over and comment on a written summary of their (children's) transcripts, and to receive/request the study findings in the form of thesis/publication/presentation towards the end of the research.

In addition to getting informed consent from the participants, I needed to consider other issues such as anonymity, confidentiality, access, storage, use and disposal of data. As for anonymity, the participants were informed that their real names would be replaced with pseudonyms and that none of their personal details would be used so that no one could identify them from their details. The children were asked to choose their pseudonyms whereas other participants were given pseudonyms. In terms of confidentiality, it was made clear that their personal details, as well as all the data to be collected, would not be shared with anyone except my supervisors. They were also informed that if a need for a transcriber emerged, the transcriber would sign a confidentiality sheet. In addition, the participants' data and personal details would be stored in a secure place where no one can access it except me.

Ethical Approval (\#24220) was granted by Victoria University of Wellington Human Ethics Committee in April 2017 (See Appendix E). Having received the approval letter from the Human Ethics Committee, a letter of permission was obtained from the school research site (See Appendix F). Before data collection started, I paid an exploratory visit to the school sites to hand out the information sheets and consent forms to the potential participants. It was also a chance to meet the community members, inform them of the purpose of the research, and answer any questions they had. It is worth mentioning that I had also previously visited the school via invitation during the end-of-the-year celebration in 2016. During that event the school operation manager introduced me to the teachers, parents, and children, noting that I 
would be conducting a research project that the community would benefit from. The school and families welcomed me warmly, and I was invited to talk to them about the benefits of multilingualism.

In the exploratory visit, the families seemed interested in participating in the study. While some parents read the information sheets and consent forms, asked about the motivations of the research and signed the sheets on the spot, others understandably wanted to discuss their children's participation with their spouses. Getting the consent forms signed was an ongoing process. I had to keep some copies with me during the observation since some parents were not given the forms or they reported losing their copies. Additionally, the participants were requested to sign more than one consent form as there were forms for adults, parents, children, and special forms for each data collection method. Participant information sheets were distributed to participants, and consent forms were signed by them. Parents and children's consent and assent were obtained. After getting consent from parents, children (less than 7), in the presence of one or both parent(s) and/or teachers (if at school), were requested to give assent. Oral assent was obtained by means of pictures (one expressing happiness and the other expressing sadness). When they agreed to participate, they pointed to the one showing happiness and vice versa. Police clearance was not needed as I was not alone with the children. This was arranged and confirmed with the Ministry of Education by my supervisor, Corinne Seals. These procedures applied to all phases of data collection.

When the observations started, both teachers and students were a bit cautious of my presence, but as time passed, they became more used to it. Their comfort seemed to be evidenced when they requested my participation in language-related discussions in the class. For example, one of the learners in site B told the teacher there was a Māori name for India, reporting that he could not remember it. The teacher provided some suggestions and told the class that country names do not change across languages. Having said that she turned to me and asked me if she was right (Fieldnotes 3 September, 2017). Moreover, the participants were regularly aware of the recording sets. At the beginning of the recording, the participants whispered when going off-topic, but also the recordings captured a lot of hushing sounds so as to notify the speakers of the recording being turned on (Fieldnotes 5 August, 2017 and 6 August, 2017). Bit by bit, the participants started to have overt discussions over the recording, for example, having conversations on how the recording set operates and reading the timer (Fieldnotes 3 September, 2017). It became clear however that the students became used to being recorded and engaged in conversations around topics that people perhaps prefer not to be recorded having 
conversations around (e.g. Excerpts 4.11 and 4.13). In these cases, I reminded the participants that they could review and delete whatever they wished, but none did.

Only one family in site A opted out of the research. In this case, I reassured them that neither their child nor any observed or recorded data for which she was present would be used in the analysis. For the linguistic reflection drawings, some parents did not want their children involved. Thus, their children were excluded from the second phase of data collection. The third phase was much more difficult. I struggled to find families who were willing to record at home. However, thanks to the school operations manager who tirelessly helped me find participants, I gathered voluntary recordings from the homes of three families.

\subsection{Conclusion}

By providing a detailed description of the steps taken throughout the research, this chapter illustrated the grounding in ethnography, and Linguistic Ethnography to be more precise. In this chapter, I justified my methodological choices and recounted the whole 'story' starting from the very beginning of how I approached the community ending with data analysis. Gaining access to the community required investing in my social capital in order to be able to approach the community through my insider friends. Once I acquainted myself with the community, I continued reflecting on my positionality as an outsider trying to build rapport with the community in order to gain an emic perspective.

This chapter presented the three stages of data collection which included observations in the HS, and interviews with parents and school staff, linguistic reflection drawings and recordings of naturally occurring interaction at home. In addition, I provided a detailed account of the participants with a special focus on the key participants who took part in all three data collection stages.

Data analysis was an ongoing process in the sense that I was transcribing and analysing as I was collecting data. The data was transcribed using Olympus AS-2400 Transcription Kit and then imported to the qualitative data analysis software NVivo12 Plus where data was reviewed and coded into relevant nodes. After that, in-depth discourse analysis was carried out resulting in the findings presented in the following chapters. I ended this chapter with a discussion of the ethical considerations that were of great importance to my fieldwork including anonymity and confidentiality of my participants' details. 


\section{Chapter Four: Language practices in the HL school}

\subsection{Introduction}

As mentioned earlier, the Indian communities in New Zealand are characterized by diversity. This diversity was reflected in the linguistic, cultural, and national backgrounds of the members of the Hindi School. Thus, a fixed realisation of the 'Indian community' did not match the superdiverse setting (Blommaert \& Rampton, 2012). The community I worked with was not only diverse, but also dynamic. Members of the HS belonged to various linguistic, ethnic, economic, professional and religious backgrounds. Likewise, some of them were former refugees. They did not share one ethnic group nor did they share one cultural pattern. Instead, they shared a passion to transmit their Indian heritage to their children and although some of them did not speak Hindi natively, they chose India's national language, Hindi, as a means of fulfilling this goal. Bearing in mind such characteristics, the HS community could not be considered a speech community nor did it form an ethnic group, but it was one that had this joint goal and was mutually engaged to achieve their goal. Consequently, I made the choice to use the CofP model (Lave \& Wenger, 1991; Wenger, 1998b) as an analytical tool in as much as the data reached the definitonal requirements.

As outlined in Chapter Two, (applied) sociolinguistic research seems to imply that heritage language schools automatically represent CofPs (e.g. Lyutykh, 2011; Oriyama, 2016; Weldeyesus, 2009) while others may qualify that it is important to critically consider if the CofP is an appropriate level. This encouraged me to problematise this fixed view of CofPs in researching HL schools. Against this backdrop, the chapter is structured in a way that allows for identifying the language practices extant in the HS as well as reassessing the suitability of CofP level of analysis to describe a diverse HL school like the HS. This chapter starts by addressing the structure of the HS and the potential of CofPs to describe and analyse the language practices in these CofPs. I also present the shared practices in the separate but interconnected sites of the HS. The chapter concludes with the distinct as well as the different practices, which suggest that the HS constitutes a 'constellation of CofPs' (Wenger, 1998b).

The HS was viewed as a meeting point for people who wished to maintain the language that would connect them with their Indian heritage and identity. From the observations and interviews held in the HS, the participants reported that they were concerned about the preservation of their Indian identity and viewed Hindi as the primary indicator of this identity. The participants also reported their hopes for the inclusion of Hindi in the New Zealand 
curriculum. Similarly, members of the HS shared distinct practices at each of the three sites such as the absence of introductory preambles and assessing whether a certain behaviour was acceptable, as shown in Section 4.4. The existence of a domain (or joint enterprise) does not necessarily make the HS constitute a CofP; however, in order to be considered a CofP, people should engage in doing things together. In the remainder of this chapter, I argue that the HS constituted a 'constellation of communities of practice' (Wenger, 1998b) rather than a single CofP. I also argue that the HS was comprised of three CofPs, as well as a 'micro-community of knowledge' (Fletcher, 2014) in which representatives from the three CofPs jointly worked for the interest of their communities. A micro-community of knowledge (Von Krogh, Ichijo, $\&$ Nonaka, 2000) is a group in which members come together from different functional groups to create new knowledge around, for instance, a service, a product, a system or a way of doing things (Fletcher, 2014). Micro-communities of knowledge are similar to CofPs. However, Von Krogh et al. (2000) explained the differences between them, noting that

[w]hile a community of practice is a place in which members learn knowledge that is embedded there, an enabling context [i.e. Microcommunity of knowledge] helps create new knowledge. The boundary of a community of practice is firmly set by the task, culture, and history of that community, but an enabling context is determined by the participants and can be changed easily. Membership in a community of practice is fairly stable, and it takes new members time to become full participants. But the many organisational members who interact in an enabling context come and go.

(Von Krogh et al., 2000, pp. 180, emphasis mine)

In the HS, when members from the school and the community around it constituted a school board to develop and create new ways of developing the school work, they were forming a micro-community of knowledge. The school board was a group of teachers, parents and family members from the three school sites who met regularly to discuss, develop, and plan for future actions. Thus, their meetings aimed to create new lines of knowledge and practices rather than learning, and those members would 'come and go' frequently. For example, Rimpy ji, a teacher at Site C, was a member of the school board when the observation started, and when she left two weeks later, a parent joined.

Furthermore, the concept of 'communities of imagination' (Wenger, 1998b) or 'imagined communities' (Anderson, 1991) has additional resonance for HL and LM research (e.g. Barkhuizen \& De Klerk, 2006; Wu, Lee, \& Leung, 2014) and for my research as well. People living in the diaspora often try to maintain ties with families and communities around them. 
They try to establish connections with communities that are "not immediately tangible and accessible" (Norton \& McKinney, 2011, p. 76). The sense of belonging or participation in an imagined community, as Anderson (1991) suggests, results from imagination. When discussing nationalism, Anderson points out that "members of even the smallest nation will never know most of their fellow members, meet them, or even hear of them, yet in the minds of each lives the image of their communion" (Anderson, 1991, p. 6). The community of imagination is central to Wenger's (1998b) discussion of modes of belonging which help produce "new images of possibility and new ways of understanding one's relation to the world that transcend more immediate acts of engagement" (Norton, 2001, p. 80). The analysis in this chapter argues that members of the HS affiliated and aligned with various imagined communities through their participation in the HS, including Hindi speakers in New Zealand and Indians in New Zealand.

In the analysis, I draw upon Wenger et al's (2002, p. 27) three defining features of CofPs, which are a 'domain' of knowledge, a 'community', and shared 'practice' to identify language practices in the HS and delve into how members negotiated meaning locally among themselves. These three dimensions are also referred to as 'joint enterprise', 'mutual engagement', and 'shared repertoire' respectively (Wenger, 1998b). These terms will be used interchangeably while providing examples from the data at both school and site levels.

\subsection{The Hindi School as a domain}

According to Wenger et al. (2002), a domain can be recognized when community members share an interest or face a problem that is not officially recognised. The group of people around the HS saw the school as an institutional space for their 'domain of knowledge'(or joint enterprise), which they identified either as an interest to transmit their Indian heritage or as a heritage transmission problem they faced with their children at home. What made this domain easy to define was the existence of established discourse patterns within the community (Wenger et al., 2002). This domain was made clear across the HS sites. The participants' discourse (in the fieldnotes and interviews) indicated that transmitting the Indian heritage and identity to the younger generation was their primary concern. Kabir, a father of three HLLs at site $\mathrm{C}$, emphasized the importance of not only speaking the language, but also passing the Indian culture to his children.

"I want the seeds to be inside them so that when they grow into their twenties and they realize hey they've got actually a huge cultural heritage which other 
people do not have ... I don't want them not to be able to have a conversation in Hindi I want them to understand the culture"

Similarly, Kaya ji, a mother of two HLLs at site C, showed her desire for her children to gain Indian cultural knowledge and identify as Indians.

"I want them to learn the culture as well at the same time and I also want them to identify with what it is like to be Indian yeah ... you know identifying with who they are + learning their mother tongue + learning the culture um opening them up to you know as they get older to travel"

Likewise, Jeff, a father of an HLL at site A, viewed joining the HS as a way to connect Indians who spoke other Indian languages.

"it will keep the language alive it will keep the culture alive and it will keep our children proud of their culture and identity like I am very proud of I am able to speak Hindi ... we've got a lot of Tamil people entered New Zealand recently and then they really want to keep culture and identity alive so they see the Hindi School as a medium of doing it"

For them, this shared interest was a "common ground and a sense of identity" (Wenger et al., 2002, p. 27) primarily by linking the Indian heritage to India's national language (Hindi) and cultures, which in turn constituted the 'raison d'être' of this domain (Wenger et al., 2002, p. 31). This connection was achieved primarily through the institutional space that provided Hindi learning services. Originally established in the 1990s, the HS was founded to connect people of Indian descent with their Indian Heritage. It served as a heritage platform that enabled people to keep close tabs on heritage and support their Indian identity in the diaspora.

The HS connected people with the Indian heritage through the weekend language classes, Indian artefact workshops, and language and culture competitions. Additionally, the HS regularly took part in nationally organised religious, ethnic, and cultural festivals and celebrations, such as Diwali, Hindi Divas, India's anniversary of independence, Bharat Bhawan (Fieldnotes 5 August 2017, 12 August 2017, 13 August 2017, 26 August 2017, 27 August 2017, 10 September 2017), following Indian cultural norms and values. Members of the HS also worked in collaboration with members of the wider (Indian) New Zealand society to publish an annual magazine called 'Darpan' (mirror) to let people know about their work and to document it. Consequently, this raison d'être defined the identity of each CofP (Wenger et al., 2002, p. 31) and connected it to the 'community of imagination' (Anderson, 1991; Wenger, 1998b) of Hindi speakers as a more recognised community whose language, Hindi, was the fourth most spoken language in New Zealand with over 66,000 speakers (Statistics New Zealand, 2013) rather than a minority language spoken by a small number of people. 
Similarly, the HS provided an Indian space that reflected Indian 'societal multilingualism' (Sridhar, 2002) and offered a domain for children to practice and be exposed to Hindi. For example, during the Hindi Divas celebration which the HS arranged in September 2017, the celebration was conducted mostly in Hindi and speakers talked about the importance of Hindi, encouraging parents to set strict family language policies by speaking only their mother tongue(s) and Hindi with their children (Fieldnotes 16 September 2017). In addition to being actively engaged in celebrating India's events, the HS was involved in other national events related to both Fiji and New Zealand. For example, Hindi learners prepared cards for their fathers on New Zealand Father's Day (Fieldnotes 3 September 2017). On another occasion, during the Hindi Divas, the presenter announced that Fiji Day would be celebrated in October and invited the audience to attend and celebrate the event with their Fiji-Indian fellows. Their regular activities and meetings were under the umbrella of a shared domain that encouraged them to contribute their insights to the practice of the community (Wenger et al., 2002, p. 29). They also worked collaboratively to develop the curriculum and textbooks. This, in turn, made them accountable for their contributions to the community and therefore to the development of practices (Wenger et al., 2002, p. 30). Thus, the domain strengthened their sense of belonging to the community.

Community members (e.g. teachers) relied on other members (e.g. parents) for help if an emergency happened. In Excerpt 4.1, one of the HS sites encountered a staffing problem as a teacher, Sun ji, had to go overseas. After the class, Sun ji waited for parents to pick up their children so that she could discuss with them the possibility that they would act as relievers for the class during her trip overseas. Excerpt 4.1 shows how Sun ji and the two parents informally negotiated how they could manage the staffing issue, even though their understanding of what the children learn was different.

Excerpt 4.1: Sun ji seeks assistance from parents (Recorded Observation)

\begin{tabular}{|c|c|c|}
\hline 1 & Sun ji: & $\begin{array}{c}\text { [addressing Avni's father] aur aapko to Hindi aati hai na? } \\
\text { [and do you know Hindi?] }\end{array}$ \\
\hline 2 & & $\begin{array}{r}\text { [addressing Yash's father] aapko Hindi aati hai: + thodi thodi ya? } \\
\text { [and do you know Hindi + a little bit?] }\end{array}$ \\
\hline 3 & $\begin{array}{l}\text { Yash's } \\
\text { Father: }\end{array}$ & I can't teach but I can + \\
\hline 4 & Sun ji: & but you can support \\
\hline 5 & Avni's & you and uh: + don't have any problem to support but you know $+/ /$ like the \\
\hline 6 & Father: & teaching is like the teaching is different \\
\hline 7 & Sun ji: & /yes, you're rightll \\
\hline 8 & & //yeah that's why + that's why we have teachers [laugh] \\
\hline 9 & Fathers: & $/[$ laugh] $] \backslash$ \\
\hline
\end{tabular}


10 Yash's father: in English + we can manage but Hindi [side-to-side hand wave, a gesture meaning No]

11 Avni's father: I liked it but + maybe //I'm not ablel

12 Sun ji: yes yes//

13 Avni's father: I'll talk with my wife if she's interested +

15 Sun ji: maybe I'll //get herl

16 Yash's father: 17

18 Sun ji: /yeah $\backslash+$ get her to get interested + just for these [three weeks] because I do often confuse with the singular + not singular + plural ++ gender + how do you say + in grammar oh + ok + and you won't have to teach grammar

When two of the parents (Yash's father and Anvi's father) arrive, she initiates the conversation by asking whether they know Hindi (lines 1 and 2). Yash's father shows willingness to assist (line 3), but he reports that he cannot teach Hindi due to his own limited ability to cope with the grammatical and morphological aspects of Hindi such as countable nouns and gender (line 16). Another alternative solution is offered by Avni's father. He suggests that he talks to his wife to see if she is interested (line 13).

Additionally, the impetus of membership in the community varied among community members from personal to societal drivers, engendering different levels of participation. The data from the observations and the interviews identified the hierarchical membership roles that emerged in the negotiation of practices. While these roles were recognised within the community, they were in ongoing construction. Teachers were seen as core members of the community for various reasons. For example, Sun ji was seen as a 'core' member by other members. Apart from her institutional role (as a teacher) that was viewed in the community as a leading role and as an authority figure (a view that derived from the wider Indian culture), Sun ji was highly engaged in the community practices. Her participation, her influence on norm building, and her role as a person to whom other members of the community referred for leadership roles gave evidence of her core status. In Excerpt 4.1, Sun ji's status as a core member in the discussion of her replacement was co-constructed in the negotiations. While attempting to assign her institutional role to other members, her initiation of the negotiation by asking about the fathers' Hindi proficiency without a preamble reflected her recognition of self as a legitimate agent to do so. Lack of preamble and rapid setting-up for problems are recognisable features of core members in workplace CofPs (Holmes \& Meyerhoff, 1999, p. 177). Additionally, other members (the two fathers in this example) acknowledged her legitimacy and responsibility to conduct the negotiation and they, in turn, participated in the negotiation. 
In contrast, although the data from observations shows that many parents were on the periphery, some parents, such as Yash's father and Anvi's father, seemed to be in a more active position. In addition to making the decision for their children to attend HS, they attended events and festivals, offering help when possible. Again, these active roles were co-constructed. Returning to Excerpt 4.1, Yash's father and Anvi's father are viewed as legitimate members who can potentially lead the classes while Sun ji is overseas (lines 1, 2 and 4). They are negotiating possible solutions for the current staffing issue (lines 3, 5 and 6). Furthermore, Anvi's father moves to a central role in the negotiations (lines 13 and 14). His suggestion to connect his wife, who seems to be on the periphery, with the school makes him a gathering point between the school and a potential teacher (i.e. his wife). Simultaneously, Sun ji acknowledges his central role as an influential connecting member in the negotiation with his wife over the staffing issue (line 15).

As illustrated in the previous example, the members' levels of participation are not fixed. They are constantly constructed. Community members can easily move in an 'inbound trajectory' (Wenger, 2010) or otherwise move back and forth in the (re)negotiations of their identities. This is the case for Sun ji as a core member, the two fathers as active members who become central in the negotiation, particularly Anvi's father, and Anvi's mother who has the potential to be moving in an inbound trajectory to a more active role in the community.

The aforementioned quotes (Kabir, Kaya ji, and Jeff's) in the beginning of this section illustrate that members of the HS CofPs shared an understanding of their domain which helped them maintain their identity through the ongoing issues they encountered (Wenger et al., 2002, p. 31), constituting a space for teaching Hindi to keep alive their linguistic and cultural identities. However, Excerpt 4.1 suggests that the members' awareness of what their domain might include varied. The data reveals that while school board members understood their work as a way by which Hindi could move to the forefront of recognised community languages in New Zealand and gain official support, the parents' desire was that their children know Hindi, for example, to connect with extended family, and other people when visiting India. The learners also had their own motivations. While some matched their parents' desires, others had their own goals such as socialising and making friends. In fact, community members' different awareness levels of what the domain may include are not unhealthy for the community since such awareness is not a requirement for shared understanding (Wenger et al., 2002). These differences serve the dynamicity of the domain and allow for negotiation (Wenger et al., 2002), 
but also these differences engender different levels of participation in the practices as presented in Excerpt 4.1.

\subsection{The Hindi school as a community}

As a community, people are committed to and care about their domain (Wenger et al., 2002). In each of the HS sites, a group of families from different linguistic (e.g. Tamil, Hindi, Telugu, Kannada), religious (e.g. Hindu and Christian), and social backgrounds (e.g. businessperson, civil servant, teacher) were committed to their domain. They not only used practices to achieve their shared goal but also used these practices interconnectedly, achieving 'mutual accountability' (Wenger, 1998a). They contributed to the coherence of their communities by their 'mutual engagement' (Wenger, 1998b, p. 73) in the weekend classes, meetings and other activities. An example of such practices was found in the members' voluntary engagement in the school. The HS teachers, teacher aides and the school board members were all volunteers who worked jointly to sustain 'community maintenance' (Wenger, 1998b, p. 74). Each of the three sites depended on "the voluntary engagement of [its] members and on the emergence of internal leadership" (Wenger et al., 2002, p. 12) as the case of Anvi's father's initiative to negotiate Sun ji's replacement with his wife in Excerpt 4.1.

Members of a CofP share distinctive cultural features that are related to the community and are instantiated in their interaction (Holmes et al., 2011). In the HS CofPs, members of each CofP shared the understanding of their CofP cultural norms, which can be opaque to outsiders. For example, my fieldnotes included comments that they shared the norms of broaching an HSrelated topic (See 4.4.2 for examples). In Excerpt 4.1, the way Sun ji started the conversation by asking the two parents about their knowledge of Hindi was quite blurred to outsiders and often to members on the periphery yet understood immediately by the men. I wrote (Fieldnotes 13 August, 2017):

I wonder why Yash's father is giving this response to Sun ji's question. I did not expect such an answer. I either missed something or there is some previous knowledge about what Sun ji is trying to say.

It is worth noting here that my commentary on the interaction derived from my status as a legitimate peripheral member rather than an outsider. In fact, my presence as a practitioner enabled me to recognise my status, and my reflection on the inside and outside during the observation gave me more access to what an insider thinks in terms of understanding more levels of meanings throughout the negotiations. However, as a novice legitimate peripheral member, I could not at first process the sequence of Sun ji's question and Yash's father's 
response. It was expected that the answer to Sun ji's polar questions, which were asked in Hindi (Excerpt 4.1, lines 1 and 2), would be consistent with the questions (simply yes or no). However, the parents responded to the questions as a continuation of negotiation of their willingness to teach Hindi (lines 3 and 5), perhaps due to their expert legitimate peripheral participation in contrast with my novice status. Thus, there seem to be locally shared histories within the community, upon which they drew in their engagement, and which allowed for the perception of their discussion as a continuation of an ongoing process within various localities of engagement (Wenger, 1998b, p. 125).

The complex and dynamic nature of the communities requires a dynamic conceptualisation of what, when, and how a person participates. That is, the engagement in the practices and participation statuses vary across activities. Such variation of participation levels suggests that members' sense of belonging is dynamic. For instance, in my fieldnotes (15 August, 2017; 10 September, 2017; 16 September, 2017) I reported that the activities pertaining to religious, national and other cultural ties involved more people than the HS activities did. My observations also indicated that some HS members, who had peripheral roles in their respective CofPs, had central roles in nationally organised activities. The reason why the status of an individual (e.g. core or peripheral) shifted across these activities could be that the HS CofPs were connected to other CofPs (e.g. religious, regional) embedded within a community of purpose (for maintaining Hindi) that was also embedded within an imagined community (for Indians in New Zealand). Thus, participation status shifted depending on the community that was activated. On the other hand, the HS activities involved 'core' members of their respective communities in the weekly classes. The HS included three sites, A, B, and C. While A and B classes took place on Sundays, C classes took place on Saturdays (See Chapter Three). Hindi weekend classes constituted a fertile environment that contributed to the strengthening of the ties between the children and their Indian heritage.

In addition to the distinctive features that community members share, members of a CofP have a common vision of their community (of practice) and talk about its value (Wenger et al., 2002). The fieldnotes and interviews reported that members of the three CofPs consistently used the term 'Hindi School' to refer to their respective communities. They also viewed the HS as a supplementary domain that helped them, as members of the 'imagined community' (Anderson, 1991) of Hindi speakers, to maintain Hindi at home. In Excerpt 4.2, Jeff is a parent at site A. He describes his view of the HS as an environment that helps him solve "the complete 
mess" of communication he experiences when trying to connect his son with the extended family (in India) over the phone.

\section{Excerpt 4.2: Jeff's view of the Hindi School (Interview)}

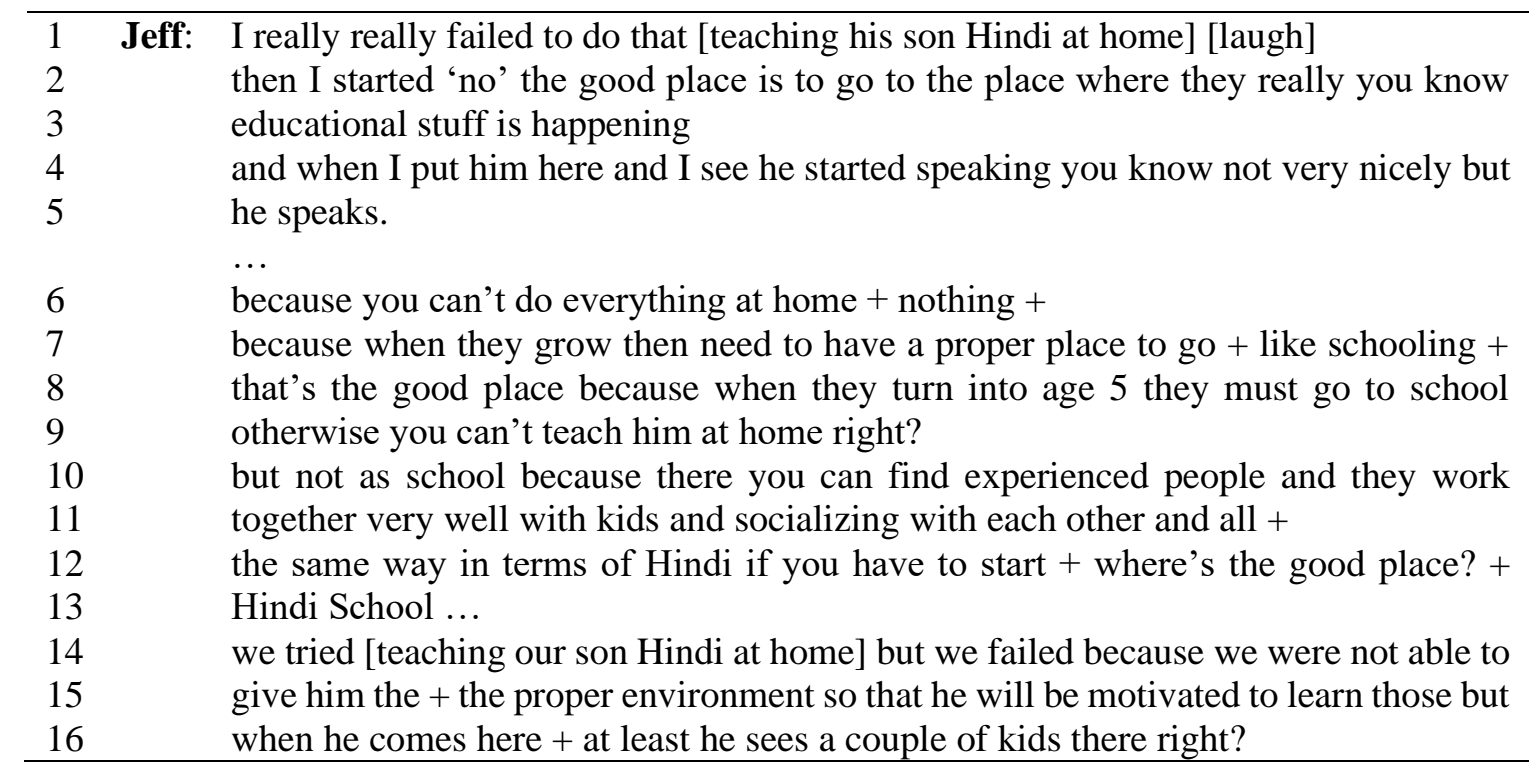

Jeff's view of the HS is constructed by what he sees as unsuccessful endeavours to maintain Hindi at home (line 1). To him, if the home efforts are not conducive to learning the language (lines $1,9,14)$, the HS is the best place as it offers language learning to which the child actively responds (line 4) due to the resources available in the school including the pedagogical knowledge (lines 2,10), a 'proper' and motivating environment (line 15), and the influence of peers on his son's language (line 16). The excerpt shows how membership in this community plays a vital role in achieving his objective as his son starts to speak Hindi (line 4). While the beginning of the excerpt might give the impression that Jeff is viewing the HS as an institution for Hindi literacy, his elaboration proves the contrary. He views the community as a space replete with linguistically experienced members who are legitimate and effective contributors to his son's progress in language learning through socialisation (lines 11, 16).

Unlike Jeff, Kabir, a parent of three children at site B, contested the idea that the children were learning Hindi. Yet, he shared the same vision regarding being a member of the community. In Excerpt 4.3, Kabir shares his motivations to send his children to the Hindi School. 
Excerpt 4.3: Kabir's comment on HS (Interview)

1 Kabir: honestly I don't think they learn any Hindi here + because like right now she was

2 talking to them everyone in English ... I think that my motivation is when they go

3 into the functions + get on the stage that gives them exposure maybe they need some

$4 \quad$ friends who they can relate to um and maybe just she gives them some homework

5 and if I say to do the homework it's- if I was to give them homework they will never

6 do it but if someone else gives them homework + they will have like teacher has

$7 \quad$ given us homework and then I can help them with them.

Although Kabir notes that the children do not learn the language at the HS because the teachers use English with them (lines 1,2), he believes that it is an environment that provides the children with activities and functions necessary for exposure to the language (line 3 ). Seemingly, this exposure to Hindi is meant to be operating as reinforcement of his language policy with the children. The field notes (5 August, 2017; 12 August, 2017; 26 August, 2017, 16 September, 2017; 23 September, 2017) and the interview with Kabir both included comments that he used only Hindi with his children. Moreover, Kabir's statement that his children may need some friends who they can relate to (line 4) indicates his focus on building the children's Indian social networks to foster their identities as members of an imagined Indian community. In lines 6 and 7 Kabir alludes to the traditional Indian ideologies that depict teachers as a legitimate authority to set homework tasks. This makes him think that his children feel committed to do homework they would not do if he were the person who assigned it. Thus, Kabir's view is not so different from Jeff's in that they view the HS as space where legitimate members (e.g. knowledgeable people, peers) are available along with other resources, linguistic or otherwise (e.g. activities, performance).

While having different views on the Hindi School, both Jeff and Kabir agreed on its vital role in supporting them to encourage their children to learn Hindi. Although Kabir believed that the HS did not offer his children the desired exposure to the language per se, he actively engaged in it since he believed in its institutional power which the children accepted and considered legitimate. Even though the HS did not operate with strict institutional conventions and adopted socialisation as a way to teach Hindi, the parents were still exercising power by organising their children's time and activities (Foucault, 1977). As shown in Excerpts 4.2 and 4.3, both Jeff and Kabir viewed the HS as an Indian space where Indian ideologies were dominant in respect to being an Indian, being a teacher, and being a parent. This space also served the parents' desire to control their children and make them more committed to learning Hindi through the legitimate power the HS wielded. This power involved making the children do what they would not otherwise have done (Foucault, 1977). 
Furthermore, the HS helped its members gain 'social capital' (Bourdieu, 1986) and expand their 'social networks' (Milroy, 1980). As shown in Excerpts 4.2 and 4.3, the HS was considered to be a place for socializing and making friends who shared the same heritage identity. The comparatively high number of students enrolled in HS, 75 students, indicated that parents were invested in being part of these CofPs and desired to benefit from the resources this domain conferred. The fieldnotes showed that the number of students who regularly attended the classes was in reality around half of the total number enrolled. This reflects that the investment in actual language practices was low, but suggests their membership in the HS could be viewed as a symbol of their Indian identity.

Interestingly, as a part of the members' vision of their community, members of the HS CofPs were aware of their multilingualism and multiculturalism and felt proud of their diversity (Fieldnotes 15 August, 2017; 10 September 2017, 16 September 2017). For example, when prepping for the Hindi Divas, the three sites got together. One of the teachers asked the attending families about their HLs saying 'how many [Hindi, FH, Tamil, ... etc] speakers do we have?'. There were 35 people in the room (including parents). To answer the teachers' questions about the languages they knew, the students raised their hands and the teacher wrote these numbers on the whiteboard, acknowledging that many people reported multiple languages. The teacher commented on their diversity that 'the Hindi School is like a mosaic' (Fieldnotes 10 September 2017).

The joint meetings the three sites arranged in different locations enabled members of the wider constellation to rehearse and demonstrate their work to other people. These meetings (and the shared domain) allowed the three separate CofPs to work in an interconnected way.

\subsection{Practices in the Hindi School}

The term 'practice' (Wenger et al., 2002) refers to 'the shared repertoire' (Wenger, 1998b) that community members develop over time as they engage in interaction (Holmes \& Meyerhoff, 1999). According to Wenger et al. (2002, p. 38) practice is defined as "socially defined ways of doing things in a specific domain; a set of common approaches and shared standards that create a basis for action, communication, problem-solving, performance, and accountability". The definition notes that ways of doing things are localised and meaningful only to members of the community. Practice does not rely on previously established rules within the community and is socially created as a by-product of interaction although it may derive from the practices of the wider communities. Practice also acknowledges previous knowledge of the community 
members (in this case the learner's linguistic knowledge) and directs them to the future (Wenger et al., 2002, p. 38). It is the practice and the way it is developed that distinguish CofPs from other communities, e.g. communities of imagination and speech communities. Members of each of the HS CofPs had socially defined rules that included greeting and addressing people, setting up conventions, and documenting work. The HS as a primary space for the CofPs was a suitable place for observing the shared practice.

The data identified several features that were shared among the three CofPs such as the absence of introductory preambles, behavioural norms to what behaviour is acceptable or unacceptable, and diversity discourse as noted at the beginning of the chapter. These overlapping practices could derive from the wider communities of imagination within which these CofPs were embedded (Wenger, 1998b; Wilson, 2011), e.g. the imagined community of Hindi speakers and the imagined community of Indians in New Zealand. This overlap could be a result of having some members in common (e.g. Sun ji teaches at sites A and C) who contributed to the development of the practice and/or the presence of a micro-community of knowledge within HS (e.g. the school board).

\subsubsection{Absence of introductory preambles}

The absence of introductory preambles is one of the indicators for a CofP (Wenger, 1998b) as members engage in conversations in different localities as if they are an ongoing one. Almost all students at the HS were returning students. This made it difficult for me as an analyst to decide whether the aspects of repertoire that I was observing had been localised in previous terms or were being developed during the term. For instance, the classes finished in all school sites at noon. Towards the end of the class (i.e. the last 5 to 10 minutes), the parents came into class to pick up their children. What is interesting is that parents did not wait for their children, for example, in the car. Instead, they came and got feedback on their children's progress, which was, according to the parents, an established rule to make the children feel how important Hindi was. The parents' presence in class also allowed for catching up with the teachers as with the case in Excerpt 4.1 which took place in the second week of the term (site A) when Sun ji discussed her absence with the parents. This behaviour was observed at all three sites as a basis for conveying the teacher's messages about any updates in the community (e.g. Fieldnotes 5 August, 2017; 6 August, 2017; 13 August, 2017) and teachers began the conversations with no introductory preambles. The case below is an example of a localised teacher-parent catch-up practice (Fieldnotes 6 August; 2017) at site A when Sun ji was telling the parents about the children's homework. 
When parents arrived, Sun ji greeted them and said: "I will send you this story for them because they know a lot of letters". Some parents responded by saying 'OK' and 'we'll work on it'. They thanked her and left.

This kind of minimal information regarding what the parents should do at home with the story was unclear to me at the beginning of the observations, but this was not the case for parents whose responses showed that they knew what to do. This localised practice was more evident to me after Sun ji had explained it to me. Below is my reflection on Sun ji's previous catch-up with the parents.

The way Sun ji talks to parents is very different from how she talks to me (about the Hindi School issues). Sun ji normally discusses things with me in detail, but with parents, she gives brief information. She doesn't even give them any information about the story or about what has been done in class. After the class when asked about this she reported that most of them knew what to do and if not, the children would tell them. In my case, she needed to give me a wider picture to give me context.

Similar to the teacher-parent interaction, teacher-student interaction was characterised by lack of preamble. An exemplar of this was the way Deepika ji started the class at site B (Fieldnotes 3 September, 2017). In this class the children continued their preparation for the Hindi Divas and New Zealand Father's Day. Over the course of two weeks, students prepared gifts/cards for their fathers. In Excerpt 4.4. Deepika ji declares the start of the class after performing the prayers.

Excerpt 4.4: Absence of preamble in the class (Recorded Observation, 3 September, 2017)

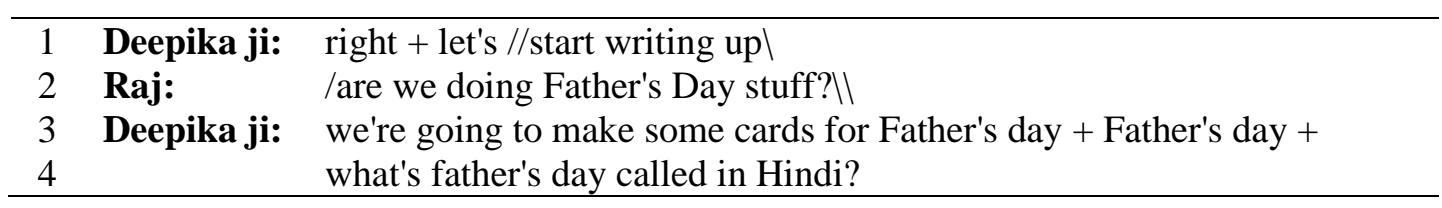

As shown in line 1, Deepika ji directs the class to start 'writing up', and although Raj seems to not know exactly which activity they are doing as they have different activities for the two events, he asks whether they are doing 'Father's Day stuff' (line 2); a question to which he gets confirmation in line 3. This set up of the activity comes to its end when Deepika ji broaches a new sub-topic, i.e. asking for the meaning of Father's Day in Hindi (line 4). The 'writing up' activity was not what I understand in educational and academic jargon, nor was it a specialised Indian jargon. It was what members of this community had developed as a localised practice. It was easy for the students to interpret Deepika ji's message and engage with their smaller groups to draw their Father's Day cards. 


\subsubsection{The ability to assess the appropriate practice}

As mentioned at the beginning of this chapter, the community members' ability to set up conventions (on what behaviour is acceptable or otherwise) serves as an indicator for identifying a CofP (Holmes \& Meyerhoff, 1999; Wenger, 1998b). When the observation started, I was a newcomer to the school with no previous experience in community language schools. I took a reflexive stance, acknowledging the different layers of meanings. It was important to draw upon additional insights from community members to analyse the practices, especially the children's behaviour. At times, the parents and I struggled with some practices and considered them misbehaviour, but this view did not apply for most of the teachers.

The class settings in the three sites were informal and children sat either at a roundtable or in small groups. There were several off-task activities and the students tended to talk whenever they wished regardless of whether the teacher was talking or not. This behaviour was quite acceptable as the teachers were trying to create an environment where the strict Indian schooling traditions were alleviated by some of New Zealand (more) tolerant educational norms. Thus, the students could do what we (parents and I) considered misbehaving, such as interacting while doing a certain task. To me, it was my teacher identity (based outside the New Zealand context) which allowed for such an assessment of the children's behaviour, but at a later stage, I came to know that the parents' assessment of this behaviour derived from the Indian norms of how students should behave. In the case below, one of the parents was helping to lead the class (at site A) while one of the teachers was absent. It was his first time engaging with the students, so he was a newcomer to the class. He directed the students to do their respective group activities, but a group of students were engaging in a gender-related conversation while reading a story. The following comment (taken from Fieldnotes 20 August, 2017) shows how this father reacted to the student's behaviour.

Aryan's father was uncomfortable with the way the students behaved. He told them to focus only on language. Then he told me that there "were about 60 students in the class in India and we obeyed the teacher, but here it is a free country and the kids do not obey. They just don't care". Later, he got fed up (with the noise and them not doing what they were instructed to do) and told the kids that they had to be obedient. He told them: "look at Lara! She is a good Indian girl. She is doing her activities".

What is interesting in this comment is the reference to India and using the label of the Indian identity as an overarching category to anchor the foundation of what is considered 'obedient' behaviour. Aryan's father's comparison between classes in India and New Zealand suggests his annoyance at the student's behaviour. He is positioning the students as 'Indians' who 
misbehave because they are raised outside of their home country. Further, describing Lara as 'a good Indian girl' is based on her 'obedience', which is the norm in Indian schools. This suggests connecting the students with their Indian identity as a common identity rather than linguistic identity. The use of the Indian identity similarly reflects the inclusion of all minority languages that the students may speak. This is comparable to Seal's (2013) study where the minority languages that Russian HL students spoke were placed under the overarching label of Russian as a heritage lingua franca.

In contrast, going off-topic was not attended to in the same way by the teacher at this site (site A). Instead, the teacher would typically contribute to the students' interaction. For example, when the students were practising a new Hindi sentence, one of the students, Summit, engaged with Aryan in an off-topic conversation on his intention not to teach his mother the new phrase. When Sun ji heard him, she participated in the conversation as shown in Excerpt 4.5 (taken from Fieldnotes 6 August, 2017)

Excerpt 4.5: Sun ji's participation in an off-task conversation (Recorded Observation)

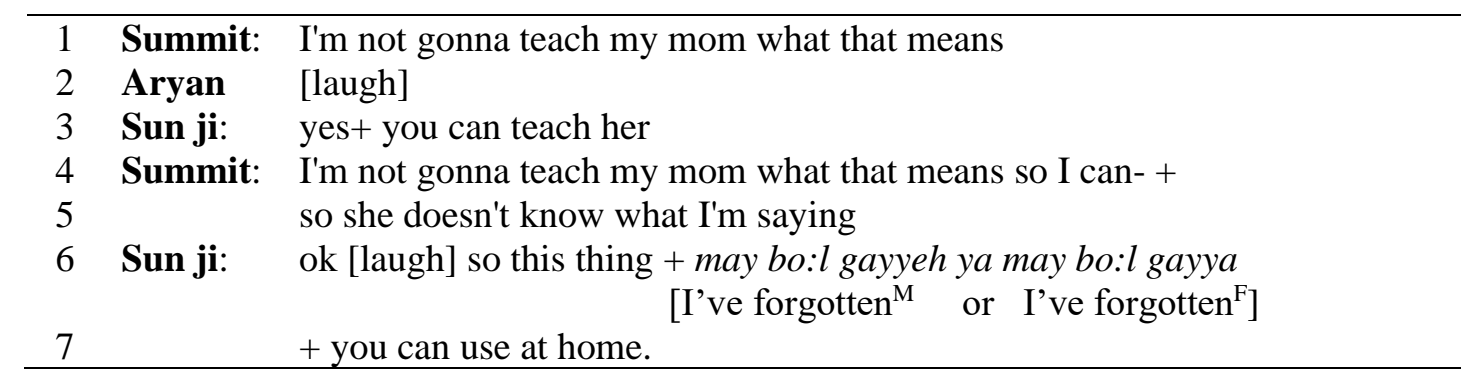

The previous example shows how Aryan's father, as a newcomer to the class, transgressed the culture of the CofP (Holmes et al., 2011). Unlike Aryan's father, in Excerpt 4.5, Sun ji contributed to the interaction by suggesting that Aryan could teach the Hindi sentence to his mother. In lines 3 and 6, Sun ji aligns with Summit's self-positioning as 'expert' (Seals, 2013) or more knowledgeable than his mother in Hindi (lines 1 and 4) by teaching his mother (line 3). Sun ji's contribution to this conversation is comparable to what Aryan's father considers as off-topic and unacceptable behaviour. However, Sun ji views the conversation as related to the CofP and she contributes to it making it of value to the children's imagined community by encouraging them to use 'may bo:l gayyeh ya may bo:l gayya' at home (lines 6 and 7). While the interaction seems to be off-topic in the educational culture, it connects to the students' linguistic identities and legitimises the sharing of knowledge as a practice in the class.

The assessment of the students' behaviour when, for example, they went off-topic, was almost identical at sites A and C, perhaps, because Sun ji taught at both sites. It was also acceptable 
for students to go off-topic at site B as was the case with the class when they practised the past tense by talking about what they did in the previous week (taken from Fieldnotes 3 September, 2017). In Excerpt 4.6, Raj abruptly went off-topic by noting that India had a Māori name. Deepika ji engaged with Raj in the conversation around the Māori word for India, and bit by a bit the other students (Agira and Adhi) engaged with them in the conversation.

Excerpt 4.6: Engaging in an off-topic interaction (Recorded Observation)

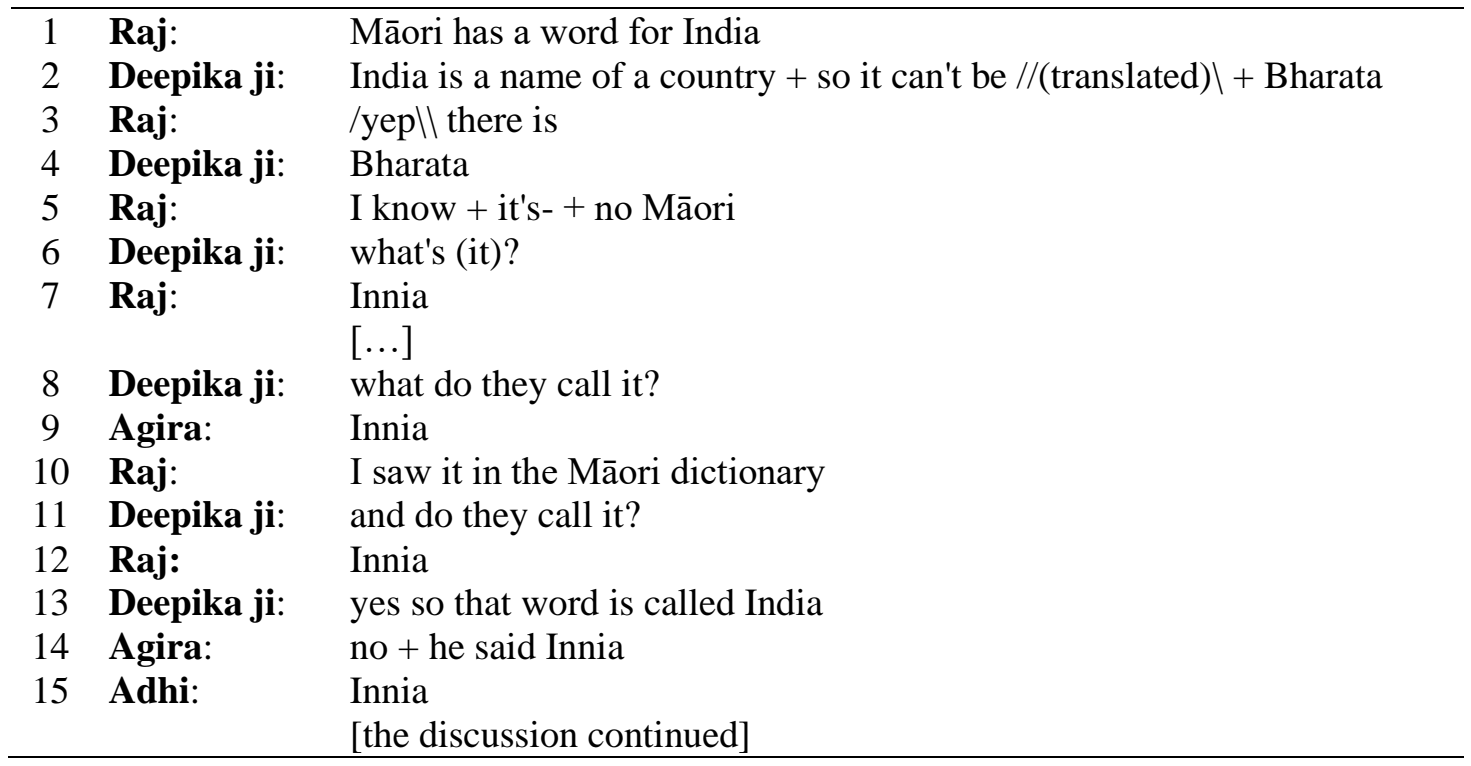

Notably, although the interaction seems to be off-topic in terms of classroom practices, it is attended to as a main point since it is related to India. Seemingly, Raj is taking an epistemological stance when introducing the new knowledge to the class (line 1). He feels that informing the class of the Māori name of India is important. This way, he shows his familiarity with the 'joint enterprise' at the HS where all India-related knowledge is valuable rather than just the language per se. He also shows a strong connection with his heritage through his interest to look up the name of his home country in a Te Reo Māori dictionary. The interaction shows how Raj starts the new topic (line 1) and how Deepika ji contributes to it as though it is the main topic. The exchange takes a dialogic form rather than a unidirectional transmission of knowledge. Raj and Deepika ji are the primary participants to co-construct the "off-topic" conversation while the rest are involved either by their 'silence' or by 'repetition' (Tannen, 2007 [1989]) showing a supportive stance for Raj (lines 9, 14 and 15). Again, Raj takes an epistemological stance in line 5 to confirm his argument, providing evidence of his knowledge (line 10). While Deepika ji's repetition of questions (lines 6, 8,11) as well as the Sanskrit name for the country 'Bharata' (lines 2 and 4) means "you must have misheard the word", Raj's stance taking comes up as an "I know" response in which he legitimises his knowledge of the 
names of his country. Deepika ji and Raj are actively engaged in the interaction where the display of knowledge about India is salient. This interaction reflects (and so does its precedents) that the assessment of behaviour in class is based on its relation to the students' Indian identity and heritage culture, which in turn constitute the CofP cultural norms.

When looking at the data, at first sight, this did not seem to be the case for all teachers. Yet, casting the analyst's eye over how the teachers at the HS assessed student's behaviour revealed a lot of identity work. For example, at site B, where language play and styling were common practice, a teacher, Savita ji, used my name to comment on their behaviour. The following comment (taken from Fieldnotes 3 September, 2017) shows Savita ji's reaction to the student's behaviour when Deepika ji directed the class to work on their Father's Day cards. The students were working on their cards while interacting. Savita ji came in, instructed them to work and left. Later, she came in again and saw them working while engaging in language play by inserting Hindi style to the phrase 'Happy Father's Day' and changing its meaning (See Excerpt 4.11). At that point, she explicitly spelt out the reason why she considered their behaviour to be unacceptable. The following is my comment.

Savita ji looked at Harry and Nayef and said in a serious tone that "Mohammed will say the Hindi community people they misbehave with the teachers. They don't listen". Harry was annoyed and said that he didn't know how to write it and they were not misbehaving; they were talking together while writing their cards.

Seemingly, Savita ji strategically used my presence as an 'overhearer' (Bell, 1984) to raise the students' awareness that others would consider their behaviour unacceptable and debatably she might have felt they had crossed a boundary. Thus, she was trying to refer to something in the environment to make the point. It could also be that my presence, a non-Indian, influenced her assessment of the children's behaviour. She might have thought about my assessment of the boys' behaviour which made her say so. Furthermore, the children's reaction to her statement demonstrated the markedness of her behaviour; Harry rejected the assessment of their behaviour as misbehaviour. In fact, his explanation that they were interacting while working can be interpreted as 'talking while working is normal in class'.

Similar to the previous cases, Savita ji adopted the same 'Indian' criteria to assess the students' behaviour, i.e. the Indian schooling traditions. Unlike Aryan's father at site A, Savita ji used the label 'Hindi community' people to anchor the students' identities. Bearing in mind that a considerable number of students were not Hindi speakers, Savita ji positioned them as members of the Hindi community since they were members of the HS. 


\subsubsection{The shared Discourse}

CofPs have a shared Discourse (Gee's (1996) Big-D Discourse) that is developed during the member's engagement in interaction. While part of this Discourse reflects the community's view on the world (Wenger, 1998b) as is the case with the member's views about the school language policy, the repertoire includes linguistic practices which distinguish that community. Community members engage in certain practices and talk about these practices. In the CofPs within the HS, the data identified major themes of their discussions on how they did things.

The most noticeable theme within the HS was diversity Discourse. Fieldnotes and interviews included examples about the way members of the three CofPs negotiated and developed their practices and Indian identity within their respective communities, and their discursive strategies were developed due to the diversity found in the HS. The HS brought families together to create common ground for their children to acquire the Hindi language and its literacy skills. It is worth noting here that since community members came from different countries, with different religious and linguistic backgrounds, the focus was on the Indian identity (i.e. Indianness), rather than other identities such as regional, national or religious ones. This focus made the HS inclusive of anyone who had direct or indirect links with Indian heritage. An example of this diversity was evident at the national level in the Hindi Divas celebration when a guest speaker announced that Fiji Day 2017 would be on October 17 and encouraged the audience to attend (Fieldnote September 16, 2017). Concerning language, in an interview, Kaya ji referred to the students in the HS as "speakers of English learning Hindi ... especially in [site C] because it's such a mixed um you know group of people".

In addition to nationality, a reference to various cultures came up in an interview with Jeff, who had strong links with the HS, when talking about the future of Hindi in the interview. He indicated that

when they [parents] don't follow the cultures + you know + some parents are from India + some parents are from Fiji + a few are from Mauritius and from South Africa + mother and father from that country where they speak in Hindi and their Hindi is priority + when they come to New Zealand mother and father don't bother to get the- teach the same culture to the children

Moreover, religious diversity was also acknowledged by Sun ji in the interview in her discussion of how the school introduces Hindi literacy to the students. She noted then I needed- I found the opportune moment to do some writing to teach them some religion scripts+ you know + they do not share one faith + so I use songs and prayers that suit all their religious backgrounds. 
The school included those of Indian descent from different countries (notably India, Fiji, South Africa and New Zealand), languages (e.g. Hindi, FH, Tamil) and religious backgrounds. Thus, it served as a uniting platform for the wider Indian community. People in the HS could be identified as Indians over a continuum, as indicated in the fieldnotes. A mixture of families at the HS was identified, including:

1. Hindi speaking families where both parents spoke Hindi and children (did not) speak Hindi,

2. other Indian language speaking families where one or both parents (did/did not) speak Hindi,

3. multiple culture families who used a repertoire of languages (e.g. a Gujarati speaking father and a Hindi speaking mother), and

4. an adoptive (English speaking) family where no parent spoke Hindi, but for whom it was important that the child attended the HS.

This diversity was often viewed as the main factor that influenced the local Discourse within the school. For example, the use of English as the primary means of communication was repeatedly ascribed to be a result of the diversity of languages found at the school sites. In turn, this belief influenced the school language policy since the common belief was that English was a vehicle through which the children could learn Hindi, and thus, using English and Hindi was unproblematic. This influence on the school language policy shaped the practices within the school where translation, referring to learners' HL, and religious practices were implemented in a way that acknowledged diversity.

Although the HS had a de facto language policy, HS members did not recognise it as an official policy. Rather, they called it 'common practice'. In Excerpt 4.7 Sun ji, who was actively involved in teaching (at sites $\mathrm{A}$ and $\mathrm{C}$ as noted earlier) and administrative roles in HS, states the outline of how English is used within the HS in general.

\section{Excerpt 4.7: The HS language policy (Interview)}

\begin{tabular}{lll}
\hline 1 & Sun ji: & the common practice is mostly being bilingual in the class and ensuring that the \\
2 & & children um and the learner is understanding what's happening and as the learners \\
3 & & progress we reduce that bilingualism um then we try to speak more of Hindi um \\
4 & & so to develop the competency so that's the general practice.
\end{tabular}

Sun ji describes the language policy of the HS as a 'common practice' (line 1). She reports that the school language policy is to start off using English and Hindi to motivate the students and to get them involved in class (line 2). They then reduce the use of English and increase the use of Hindi (line 3) to allow for more exposure to Hindi so that they can develop the learner's proficiency (line 4). Using English included translation, which was an integral part of the 
practice in the three CofPs within HS. But the motives behind translating Hindi into English varied due to, as Wenger (1998b) states, the lack of mutual engagement across the HS sites. The data showed that recasting, summarising and rephrasing Hindi were generally used for linguistic accommodation purposes (Giles \& Coupland, 1991). In turn, the more knowledgeable members of a CofP (e.g. teachers) accommodated their linguistic behaviour to the less knowledgeable ones' dominant language (English) which served as a way of achieving “community maintenance" (Wenger, 1998b).

As mentioned earlier, Sun ji taught at sites A and C, with Kaya ji helping her at site C, whereas Deepika ji taught at site B. In Excerpts 4.8 and 4.9, I demonstrate how using English as a language policy was a shared practice in these sites. I also show that the views towards this practice were expressed differently across the sites, and how members of each site shared the same views about their way of doing such a language policy.

Using English within the HS was motivated by acknowledging the learners' identities to get them involved in the learning process. The way this motivation was expressed at site $\mathrm{C}$ is shown in Excerpt 4.8. Kaya ji was an active member at site C. She was a parent of two students, but also a teacher aide with Sun ji in class. In the interview, Kaya ji elaborated on their motivation at site $\mathrm{C}$ to use English.

Excerpt 4.8: Kaya ji justifies using English in class (Interview)

\begin{tabular}{cll}
\hline 1 & Kaya ji: & because the kids speak English predominantly \\
2 & & I think sometimes it's easier to give instructions to them in English \\
3 & and potentially you may give the instructions in English \\
4 & and maybe translating it in Hindi might be a good idea you know \\
5 & just ( ) they understand but mix- mixed is fine mixed is ok \\
6 & {$[\ldots]$} \\
7 & if we bring them to school and say to them hey when you get into school for the two \\
8 & hours speak in Hindi, there won't be any communication. \\
9 & do you know what I mean? They will just stop communicating at all + \\
10 & so I'd rather they talk and then and then pick up something at the end of the day \\
11 & then coming to class. \\
12 & if I said to them hey as soon as you get into the class start speaking in Hindi, all the \\
13 & communication will stop because none of them are comfortable to talk in Hindi, you \\
14 & know, and have a fuller conversation especially with the younger ones. \\
\hline
\end{tabular}

Kaya ji notes that since the learners speak English (line 1), the strategic use of English translation helps them understand (lines 3, 4). She also refers to Sun ji's idea of being bilingual in class (Excerpt 4.7, line 1) noting that the learners she has at site $\mathrm{C}$ understand Hindi (line 4). Kaya ji makes her point clear that imposing a strict policy in the classroom would detract from their willingness to communicate (lines 7, 8, 12), which corresponds to what Sun ji refers to 
ensuring that the children um and the learner is understanding what's happening (Excerpt 4.7, lines 1, 2). Moreover, Kaya ji's restates Sun ji's point that English is used to develop the learners' proficiency in Hindi (Excerpt 4.7, line 4) when she refers to building the learners' knowledge and the positive attitude towards the school as the foundation to keep them connected to the school (lines 10,11). The different articulation of Sun ji's ideas (presented in Excerpt 4.7) found in Kaya ji's interview provides an interesting example of how members of the CofP talked about the school language policy as a shared practice.

In contrast, the views were different at site B. In Excerpt 4.9, Deepika ji has a similar stance regarding the use of English, but with different reasoning.

Excerpt 4.9: Deepika ji describes the HS language policy (Interview)

\begin{tabular}{|c|c|c|}
\hline 1 & Mohammed: & do you have a language policy in Hindi School? \\
\hline $\begin{array}{l}2 \\
3 \\
4 \\
5 \\
6 \\
7\end{array}$ & Deepika ji: & $\begin{array}{l}\text { not really }+ \text { that's an area I am working on with the team }+ \\
\text { I really want them to value English that the- where we value the language } \\
\text { they're bringing to teach the new language. } \\
\text { because some of the teachers strongly believe we need to speak Hindi in } \\
\text { Hindi School but my perception is different. } \\
\text { I see. }\end{array}$ \\
\hline $\begin{array}{l}8 \\
9 \\
10 \\
11 \\
12 \\
13\end{array}$ & Deepika ji: & $\begin{array}{l}\text { if we speak just in Hindi children don't understand what we're saying and } \\
\text { learning can become very difficult. } \\
\text { so I want the idea of valuing the languages that they already know to teach } \\
\text { the new language. } \\
\text { and they come from all vast language background settings so what I } \\
\text { normally prefer is to use English as a medium of teaching so that helps. }\end{array}$ \\
\hline
\end{tabular}

In a response to my question about the school language policy (line 1), Deepika ji reports the absence of official language policy, adding that the team is developing a language policy for the school (line 2). Despite the lack of official language policy, Deepika ji asserts that the policy they adopt is to value the learner's linguistic knowledge (lines 3, 4). She highlights the value of what the children bring to class in language learning (lines 4,7), noting that the school language policy is not restricted to English and Hindi (line 10). To her, the learner's entire linguistic repertoire, including their HLs, are valued (line 10,11) because this will facilitate learning Hindi (line 9). Deepika ji's idea of valuing the language's that they already know and referring to the learner's mother tongues suggest a wider perspective regarding the school language policy. Such a perspective describes the concept of 'translanguaging' (García, 2009), which suggests that the students used their full language repertoires to demonstrate a full understanding of what was being taught (García \& Li, 2014, p. 92). 
The HS language policy so far suggests that while in bilingual education teachers are described as "servants of the system" (Shohamy, 2006, p. 79) or "the final arbiters of language policy implementation" (Menken \& García, 2010, p. 1), teachers are often responsible for their roles as 'policy makers' (Lo Bianco, 2010; Menken \& García, 2010) as well as implementers of those policies in HLE.

In addition to the shared Discourse, the HS CofPs shared a linguistic repertoire, including the members' tendency to set up norms for their practice, creating linguistic boundaries between them and others, and censoring practices. This is illustrated in the following sections.

\subsubsection{Terms of address (all sites): Set-up, repertoire policing}

Since most of the children at the HS were returning students, teachers tended not to explicitly set up the norms for their respective CofPs. Instead, they adopted a policy of tolerance regarding the shared linguistic repertoire. Norms set up was not exclusively the teachers' role, however. Rather, any member of the community (e.g. students) could take that role. The process of setting up the norms was negotiated among members in the form of repertoire 'policing' (King, 2011). One of the cases captured during data collection was the addition of the word 'ji' to show respect when addressing teachers and parents. This occurred during the first week of the term when Sun ji seized the opportunity for a teachable moment when some forgot a story they had learnt previously and focussed on how to say "I forgot it". After that, she proceeded with counting in Hindi. While Sun ji was counting, Emy, who was a newcomer, aged 5 years, interrupted her to ask about her name. Excerpt 4.10 (taken from Fieldnotes 6 August, 2017) shows the flexible way Sun ji dealt with Emy's question as well as Summit's repertoire policing over Sun ji's response.

Excerpt 4.10: $J i$ : norm set up and policing (Recorded Observation)

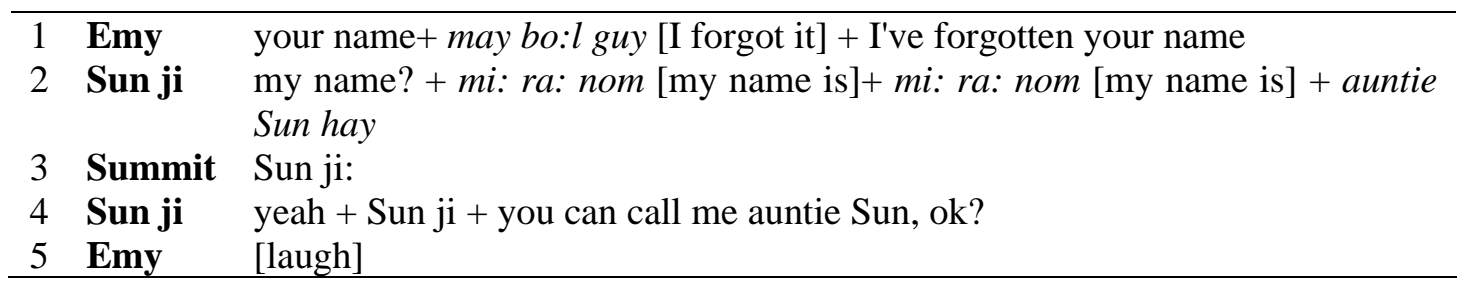

In the excerpt, Emy makes use of the newly learnt phrase to ask for Sun ji's name (line 1). While the normal address form for teachers within HS, which derived from the Indian culture, was adding ' $j i$ ' after their names, Sun ji provides another word 'hay' (line 2), since it was common in the Indian cultures that people use ' $j i$ ' after their names. This response makes Summit interfere to provide what he believes is the correct way of addressing the teacher (line 
$3)$, i.e. the 'normal' word used in the school. Summit considers himself a core member who is eligible to contribute to the conversation, taking an epistemological stance by recasting Sun ji's response (line 3). This encounter shows that members of the CofP were effectively censuring the language used among themselves. In turn, this encounter seems to enrich Summit's capacity pertaining to addressing teachers as the following comment (taken from Fieldnotes 20 August, 2017) suggests

Aryan's father was directing students to do what Sun ji planned for them. He asked them about what they did last week. They started telling him what they did. When Summit mentioned Sun ji saying "Sun hay", Aryan interrupted him saying "hey, say Sun ji" which is more respectful. Aryan was acting as a teacher as he later proudly announced: "we are teachers, we are teachers".

Interestingly, Aryan plays a core role in correcting what Summit says using the informal interjection 'hey' to express annoyance followed by the imperative 'say' for explicit instructions. Seemingly, he sees himself as an eligible person to perform repertoire policing over the other's language. The way CofP members negotiate their practices is not agreed upon. One can find some practices that are contested by members. Such behaviour within the CofP partially reflects the development of the shared repertoire and could capture a glimpse of this development through repertoire 'policing' as shown above. This finding supports Lo's (2009) findings that heritage language teachers of Korean were more indulgent than Korean children regarding the learners' failure to use honorifics.

\subsubsection{Namaste: Creating boundaries and repertoire policing}

In addition to setting up norms and repertoire policing, the data showed that members of the three CofPs created linguistic boundaries between them (as insiders to their respective communities) and the others (i.e. outsiders). This theme was consistently emerging in the way CofP members greeted their respective CofP fellows by using the Indian greeting 'namaste', accompanied by cupping both hands together and bowing. Yet, it was noticeable that this kind of greeting was a localised practice vulnerable to transgression by outsiders and newcomers. At the beginning of the observation, there were times when younger members or newcomers used 'hi' or 'good morning' to greet others, but this violation of the greeting norms was censored by others, thus it faded away over time. One of the instances was in the first week of the school term at site C (Fieldnotes 5 August, 2017). When Robin, Rose and Jack arrived at class, Rose and Jack greeted the class with 'namaste' while Robin said 'hi'. Interestingly, Max replied by saying 'namaste' instead of saying 'hi'. Noting that Robin was a returning student, 
he was expected to use the in-group greeting but as he failed to meet this expectation. Max implicitly corrected Robin's greeting by recasting the greeting. My commentary on this was:

What is noteworthy is Max's response to Robin's greeting. It seems that he's trying to teach or remind Robin of the greeting conventions in the school.

As the term passed the members of the CofPs used 'namaste' more consistently to greet one another. They were also able to recognise whether a person was an insider and greet them accordingly. Thus the children were creating boundaries between them and others in terms of membership in the community (of practice). As an observer, I was seen as an outsider at times and so were many parents. This view influenced the way the children greeted us. An illustrative example in this regard occurred when the three school sites assembled towards the end of the term, as my Fieldnotes on 10 September, 2017 indicated:

Children greeted each other and teachers (and school board members) with namaste. Yet, they greeted me and some parents saying 'good morning' or 'hey/hi' unless we initiated the greeting with 'Namaste'. It seems that they do not see us as members of their community.

Arguably, members of the CofPs adopted these strategies due to their desire to maintain their community by protecting the outer face of the community, the shared repertoire. Thus, they censored the practice to reassure themselves of the internal bonds they had. They also refrained from using their shared repertoire with others to index their outsider status because they know who belongs to the community and who does not (Wenger, 1998b).

The data presented so far illustrated a glimpse of the culture of the CofPs within the Hindi School and how members of these communities shared a set of norms that newcomers and outsiders were not familiar with. As presented, there were overlapping practices among these CofPs and the CofPs at sites $\mathrm{A}$ and $\mathrm{C}$ in particular. Yet, there was "diversity internal to practice and defined through mutual engagement" (Wenger, 1998b, p. 128) as was the case of Deepika ji and Savita ji when responding to off-topic sequences. By and large, this diversity in the practices resulted in a final and desired outcome, which was "being Indian". We have seen how members of a CofP used general words (e.g. letters, write-up) in localised settings. They also used labels (e.g. Indian, Hindi community people) to create a sense of belonging with the CofP and the imagined Indian communities. We have also seen the teacher's active involvement in conversations (that educationalists would describe as off-topic) because these conversations were related to the students' national and cultural identities.

Having discussed the practices shared across the HS, I now turn to the practices identified as unique features of a certain CofP. 


\subsubsection{Unique practices}

As noted earlier, Wenger (1998b) suggests that the lack of mutual engagement among communities results in the emergence of unique practices for each community. The same applies within the HS where the lack of mutual engagement among members of the three sites created boundaries between the CofPs and resulted in a unique linguistic repertoire at each site. In what follows I demonstrate the major themes for the three CofPs regarding the distinct repertoire that each community had.

\subsubsection{Language play at site B: Humour, translanguaging and styling}

In the 5th week of the school term, Deepika ji divided the class into groups to make artefacts for their fathers as Father's Day gifts. Deepika ji directed the groups to do their activities. I sat with a group of boys (Raj, Harry, Big A, Rohan and Nayef) when Deepika ji asked them to work on their Father's Day cards (as introduced previously in Excerpt 4.4). Among the cards, Rohan's card captured the attention of the others as he wrote 'Happy Father's Day' using Hindi transliteration (Figure 4.1).

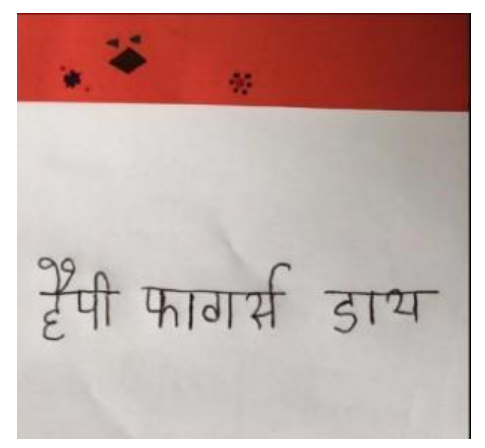

Figure 4.1 Father's Day card

As Deepika ji went around to see what the children were doing she read the card and told Rohan that he should correct some spelling mistakes. Then she asked him to write Happy Father's Day in Hindi ("pitri Divas shubh ho") instead. At that point, the boys turned their attention to the card and started to read it playfully. Doing so, they pronounced the written phrase in various ways, combining features from both languages, English and Hindi, as shown in Excerpt 4.11.

\begin{tabular}{cll}
\multicolumn{2}{c}{ Excerpt 4.11: Father's Day language play (Recorded Observation) } \\
\hline 1 & Harry: & happy- + happy Father's Day! + happy fagger's day \\
2 & & [boys laugh] \\
3 & Nayef: & fagger's day + fagger?! \\
4 & Rohan: & it's haPpy fagger's day [doing Indian-accented English] \\
5 & & [boys laugh] \\
6 & Nayef: & is it actually haPpy father? \\
7 & Harry: & [laugh] look at it \\
8 & Big A: & Happy fagger's day \\
9 & Nayef: & I can't read //it (though)
\end{tabular}




\begin{tabular}{lll}
10 & Rohan: & /it's HaPpyl\ fagger's day \\
11 & & [boys laugh] \\
12 & Nayef: & fagger! + I misheard you ( ) oh \\
13 & Rohan: & die + this is not even day + this is die \\
14 & & [boys laugh] \\
15 & Harry: & Great \\
16 & Rohan: & [low voice] happy fucker happy \\
17 & & [boys laugh] \\
18 & Rohan: & happy fathe::r \\
19 & Nayef: & it should be happy fa:theR \\
20 & Harry: & [laugh] yeah + happy fathers die ${ }^{5}$ \\
\hline
\end{tabular}

Excerpt 4.11 shows the boys integrating translanguaging (García, 2009) and styling (Coupland, $2007)$ to perform their language play. The use of the Devanagari writing system along with the playful pronunciation of the written phrase with an Indian accent (lines 4, 6, 10, 18 and 19) arguably show that the boys are using their full linguistic repertoire to navigate between their Kiwi and Indian identities as well as doing facework. Additionally, lines 1, 3, 4, 8, 10, and 16 include the production of swear words for humour ${ }^{6}$, a feature of performing masculine identity (Wilson, 2011). This kind of humour through language play can be interpreted as a way to avoid the embarrassment caused by the mistakes Deepika ji highlighted in Rohan's card. Harry initiates the language play focusing on 'fagger' as a replacement of 'father' (line 1), Rohan's contribution to the humour (line 4) is a playful manipulation of his linguistic repertoire. He initiates a styling play where he reproduces the phrase with an Indian accent. Seemingly, this language play involves the boys' shared resources whether linguistic or otherwise (e.g. Kiwi, Indian, and masculine identities) which, as Belz (2002, p. 59) suggests, may index resourceful and creative displays of the boys' multi-competence.

In this example, two points are relevant to the discussion of the CofP's shared repertoire. First, they used swearing in language play with minimal repertoire policing. Perhaps, the fact that the students in this group were all boys makes their masculine identities more dominant. So, breaching the norms (i.e. swearing) receives Nayef's attention in the form of exclamation (lines 3 and 12). Second, while Nayef's (genuine) question technically requires a yes/no answer (line 6), Harry's laughter and an invitation for Nayef to read the phrase (line 7) are an emblem of the shared linguistic knowledge they had. In line 9, Nayef's overt announcement that he cannot read the phrase refutes Harry's assumption about the shared knowledge. Interestingly, this announcement receives no laughter or mocking. Instead, Rohan reads the phrase (line 10),

\footnotetext{
5 This could also be 'happy Father's Day' stylised in Kiwi English for which Harry uses his linguistic knowledge of the Kiwi accent to make humorous, aligning with Rohan in the language play.

6 They are possibly also (naïvely) engaging in a derogatory pun and homosexual slur. They know it is improper and they should not say it but they, perhaps, do not understand what it means.
} 
perhaps, to make clear how he intends the phrase to be read or to bridge the gap in Nayef's knowledge.

As my Fieldnotes on 3 September, 2017 also recorded, the class engaged in various activities ranging from doing Father's Day artefacts to reading Hindi stories, singing, and talking about India's location on the world map. The class had a map and discussed general geographical points. Among these points, they discussed what other countries one could visit when travelling from New Zealand to India. Savita ji asked the class whether they travelled to India and what countries they stopped at on the way. Several responses were given as per the children's experiences, including Dubai and Abu Dhabi in the UAE. This discussion was conducive to language play which was initiated when Nayef said that he went to Abu Dhabi. My comment on this encounter is as follows (Fieldnotes September 3, 2017)

Once Nayef mentioned Abu Dhabi, the boys repeated the word playfully by adding the Indian accent to it. Rohan pronounced it in a mock Indian fashion as 'abu dHa:bi:', Big A contributed further, styling it as "Ipud abi:, and Nayef shifted the word into "iPod". This playful repetition of the word encourages other children to get engaged in the play, e.g. Raj laughed and continued the play saying "iPod on the headers"

The previous examples clearly show the children's playful behaviour when coming across nonHindi words. They used language as a resource for humour to do identity work. Excerpt 4.11 shows that members of the community mitigated repertoire policing in favour of masculine identity construction. It also suggests that language play and humour were localised within the practice, and when a member showed they had insufficient knowledge, learning replaced humour. Similarly, the second example is evidence of the presence of Indian identity in language use. The boys' tendency to add the Indian 'flavour' to the word Abu Dhabi reflects their sense of belonging to the community (of practice) which can be seen as a mediation between the Kiwi and Indian identities. The pattern of creative language play in the above examples started from styling the given word (with a mock Indian fashion) and then moved beyond that to match the word with another English word. This pattern can be interpreted as an index of the negotiation of their Indian and Kiwi identities.

\subsubsection{Gender talk at site A}

Before discussing gender talk as a unique shared practice of the CofP at site A, it is noteworthy that gender is an important grammatical issue in Hindi. Gender is seen as one of the challenges that people encounter, as Aryan's father noted at the beginning of this chapter (Excerpt 4.1). Although gender in grammar was introduced to the children at the three sites as an integral part 
of language where men's language was slightly different from women's language, members of site A extended gender talk beyond language borders to engage in gender in society talk.

In the second week of the term, Sun ij asked the children to tell a story. As they were hesitant to raise their hands, she called some of them by name, asking them to tell a story they had previously learnt. When she asked Summit he reported that he could not remember it. Then she shifted from storytelling to teaching them how to say 'I've forgotten' in Hindi (Fieldnotes 6 August, 2017). Sun ji introduced may bo:l gayya [I've forgotten ${ }^{\mathrm{M}}$ ] and may bo:l gayyeh [I've forgotten $\left.{ }^{\mathrm{F}}\right]$ and practised it as a class with the emphasis on gendered language. Discussing gender in this sentence generated more metalinguistic questions that seemed to have no convincing answer for Summit. Excerpt 4.12 was recorded while the children were practising saying the phrase in Hindi.

Excerpt 4.12: Introducing gendered language (Recorded Observation)

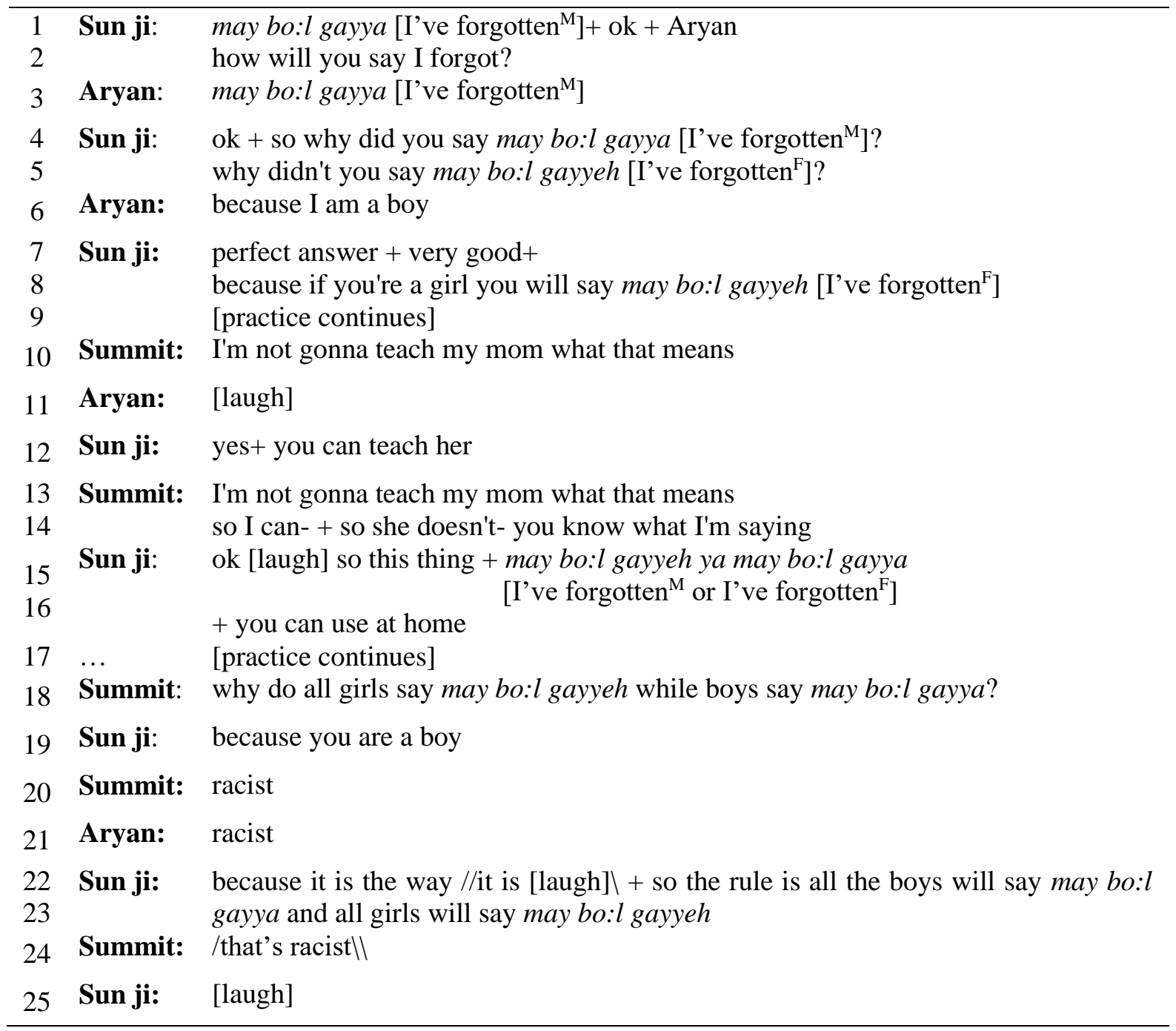


Introducing gender-in-grammar explicit knowledge to the children seems to unleash the children's passion to question differences about gender in society. Sun ji's metalinguistic questions (lines 4 and 5) are meant to check Aryan's understanding of gender in grammar. These metalinguistic questions lay the basis for Summit's further inquiry (line 18) which receives a 'sexist' answer (line 19) as the two boys perceive it (lines 20 and 21). The metalinguistic questions posed about gendered language can be arguably seen as 'thinking together' (Pyrko, Dörfler, \& Eden, 2017) about the practice they shared and identify with (Wenger, 1998b).

The negotiations around gendered language within the community reveal that identity is also negotiated. Summit's question along with labelling Suni ji's answer as 'racism' may index the children's Kiwi identity where gender differences are not often discussed. However, specifically for Summit, the intention not to teach the Hindi sentence to his mother (lines 10 and 13) may be explicitly positioning his mother as a non-Hindi speaker (because she was not a member of the school and spoke Kannada only) or that he and his father had the higher status in Hindi (noting that his father was an active member in the school who spoke Kannada and Hindi). Consequently, Summit's construction of his Indian identity in relation to knowing Hindi (as compared to his mother) seems to have shifted to a Kiwi identity when gender emerges, where differences between gender are unaccepted. Summit addresses his interlocutors' epistemological stance by giving an incomplete sentence with 'you know what I'm saying' (line 14) as an end to it. Thus, he evokes shared knowledge they had as a community, seemingly, as an attempt to foreground the CofP membership identity.

Gender talk continued in the community even if the gendered language did not. For example, two weeks after the gendered language discussion, Summit's father and Aryan's father temporarily replaced Sun ji. The children were given time to do some readings, but as usual the boys (Aryan, Summit, John) engaged in an off-task discussion regarding their levels at their state school. During this discussion, Dev, the youngest student at site A, joined the conversation. When the boys made fun of him, Lara stepped in to stop them. The mocking, at first, began due to a misunderstanding of what Dev said then moved to gender issues. Excerpt 4.13 (taken from Fieldnotes on 20 August, 2017) provides an example of how gender talk went beyond gender in grammar to gender in society. 
Excerpt 4.13: Gender talk at site A (Recorded Observation)

\begin{tabular}{lll}
\hline 1 & Dev: & I'm 8 \\
2 & Aryan: & [laugh] \\
3 & Summit: & [laugh] \\
4 & & she- he doesn't ( ) even do maths + \\
5 & & he only does English + and he's on A2 \\
6 & John: & uh A2? \\
7 & & [Aryan's father directs them not to go off-task] \\
8 & & [school levels discussion continues] \\
9 & Dev: & come on [short and high voice] \\
10 & John: & why do you sound a little squeaky? \\
11 & & why do you sound a little squeaky and tired? \\
12 & Summit: & oh sh- he's sick + \\
13 & & she he vomited \\
14 & Aryan: & oh! you don't call him she [angrily] \\
15 & Lara: & how do you know? //how do you know about ( ) $)$ \\
16 & Summit: & /you said + she vomited and my mom $($ )川 + \\
17 & & you said she not he [noise] ( ) I just heard you \\
\hline
\end{tabular}

Summit repeatedly refers to Dev as a girl (lines 4, 12 and 13) based on the weakness and pitch of his voice. Although it starts in line 4, the children seem to identify it as a slip of tongue. When Dev utters 'come on' (line 9), making a questionably 'squeaky' sound (lines 10 \&11), Summit's continuous reference to Dev as a girl is contested by Aryan and Lara (lines 14 and 15) which makes him justify his intentional use of the feminine pronoun in lines (16 and 17).

Interestingly, this kind of gender talk indicates that identity is at play. The way that Summit referred to Dev along with the opposing stance discursively expressed through Aryan and Lara's rejection shows the emerging nature of gender talk within the community as contributed here by the boys and girls of the class. One can also take such a conversation as evidence of repertoire policing within the community as Summit violated the rules set in this group. However, when focussing the analyst's gaze on gender talk in this section, we can notice a shift in Summit's stance. While he took an opposing stance against the essentialist view of gendered language, labelling it as 'racist' in Excerpt 4.12, in Excerpt 4.13 he was challenged due to the stance he took in favour of these essentialist views. Summit seemed to be in a situation where he was trying to reconcile his Kiwi and Indian identities (if we accept the more dynamic understanding of gender in society is aligned with his conceptualisation of New Zealand attitudes to gender).

All in all, the HS represented an institutional space for a group of families around it who shared a joint enterprise, representing related but distinct CofPs. The existence of three school sites, with members who did not mutually engage in the practice, led to diversity in practices and boundaries between the three sites (Wenger, 1998b). Wenger's (1998b, pp. 126-127) approach 
allows for capturing these kinds of differences. He posits that very large configurations (e.g. speakers of a language) and even small configurations (e.g. schools) that have these practice boundaries can be viewed as constellations of communities of practice. The reasons why the CofPs within the HS were viewed as related to one another in this constellation were that these CofPs shared a domain, members who faced similar conditions, and most importantly they had overlapping styles or discourses (Wenger, 1998b, p. 127). Within the constellation, one can find similarities and subtleties in the practices. Members of each of these CofPs shared a practice that might match or differ from those of the other two CofPs. In this regard, Wenger (1998b, pp. 128-129) argues for two kinds of diversity in practice within constellations; namely, diversity internal to practice and defined through mutual engagement and diversity caused by boundaries and stemming from lack of mutual engagement. This diversity was observed within the HS CofPs.

I do not claim that these three CofPs are the only possibles ones within the HS, but these are the three which emerged in the data I researched. However, unlike the Russian (Lyutykh, 2011) and Japanese (Oriyama, 2016) HL schools, we cannot assume that the HS represented a single CofP. The differences in practice indicated the boundaries between these different CofPs even though they shared a domain and a school board which Deepika ji referred to as 'the team' (Excerpt 4.9, line 2). Deepika ji reported elsewhere in the interview that 'team' is the school board, defining it as a group of members of the Indian community who actively participated in the HS functions (e.g. teachers and parents). The school board, or the team as Deepika ji called it, can arguably constitute a micro-community of knowledge (Fletcher, 2014) in which representatives from the three CofPs within the HS who came together for planning and administrative work. Clearly, there is complexity, and this reminds us to avoid assumptions and fixed conceptualisations.

\subsection{Conclusion}

In this chapter, I described and analysed the practices within the community-based Hindi language school and the communities attached to it through the concept of "community of practice' (Lave \& Wenger, 1991; Wenger, 1998b). I demonstrated how a group of families came together at the HS. I argued that each of the school sites constituted a unique CofP and these three CofPs were interconnected within a wider 'constellation of communities of practice' (Wenger, 1998b), which emerged as a result of (1) the absence of the mutual engagement across all three sites, and (2) the existence of 'micro-community of knowledge' (Fletcher, 2014) among the three school sites. Further, I argued that the students' 'sense of 
belonging' and membership in this constellation of CofPs influenced their language ideologies towards the 'heritage lingua franca' (Seals, 2013), Hindi, which in turn contributed to the construction of their multilingual identities as bi- and multilingual speakers in an Indian community. These arguments were discussed with supporting evidence concerning the members' commitment to the 'domain' (i.e. maintaining the language and the Indian identity), the existence of a unique institution for fulfilling this commitment, and the voluntary investment in the 'community' in their respective site. That is, some members of the Indian community were highly involved in the school activities through the teaching of Hindi and taking part in activities organised within the school and/or at the national level. Furthermore, the HS was viewed as an emblem of the Indian identity. The parents were actively making decisions for their children to attend the, and the children seemed invested in the practice. There was also a shared repertoire for each of the three CofPs. This repertoire included both how they enacted the practices and the way they talked about them. The shared repertoire in the CofPs was both agreed upon and contested by members, e.g. as when to speak English, when to speak Hindi, and who was accepted as a teacher etc.

I showed how members of a CofP used discursive practices to create localised meanings. I also showed how their evaluative discourse derived from an Indian national/cultural identity. For instance, the teachers became involved in off-topic conversations with the students when the conversations were related to any aspect of India. Even though these conversations were attended to as off-topic as per an educational lens, their connection to the students' national, cultural or linguistic Indian identities legitimised them as relevant to the class. I further demonstrated how members of the community (mostly teachers and parents) used labels (e.g. Indian, Hindi community people) to position the students as legitimate group members and to emphasize their national and CofP membership identities respectively. Thus, the shared practices in the HL school CofPs made visible the language ideologies that the students experienced, which, as I argue in the next chapter, influenced their identity negotiation and development.

Finally, the data from the observations and interviews provided evidence that the HS included three separate CofPs interconnected within a constellation of communities of practice. To extend this further, we can argue that this constellation was embedded within a community of purpose (for maintaining Hindi) and overlapped with a community of alignment (for maintaining community languages in New Zealand). Again, these communities fell within both a community of imagination (for Hindi speakers in NZ), and a community of imagination (for 
Indians in New Zealand). It was not an easy task to identify whether a certain practice had developed as a result of mutual engagement or whether it had been adopted from the wider communities a CofP was embedded in (similar issues are discussed in King, 2019). This issue needs to be investigated further in an ethnographic endeavour with longer observation.

As noted above, identity is at the heart of these discussions and continues as a focus in Chapter Five. 


\section{Chapter Five: Language, capital and identity construction: Indexicalities}

\subsection{Introduction}

In the previous chapter, I analysed observation and interview data to explore the discursive practices within the HS through the lens of CofPs. The analysis captured a glimpse of the shared repertoire which developed over time. While the repertoires included shared practices across the three CofPs, some other practices were unique to specific sites. Most importantly, the shared 'Big-D' Discourse in the HS revealed language ideologies towards not only Hindi, but also the potential other languages the learners had at their disposal. Additionally, although Hindi was not necessarily the heritage language of most of the students, the HS members recognized the importance of Hindi as a 'mother tongue' as the language spoken by Indians.

The current chapter expands the investigation of the influence of CofP membership on HL students' multilingual identity development as represented in the children's linguistic reflection drawings. Specifically, it explores how HL students represent and negotiate their multilingual identities, as well as their language ideologies towards their languages. The analysis of the learner's linguistic reflection through their descriptive accounts is informed by the concepts of 'indexicality' (Ochs, 1993) and 'investment' (Norton, 2010, 2013; Norton Peirce, 1995). I begin the chapter by introducing linguistic reflection drawings and their value in collecting data from young participants. Next, I demonstrate how HLLs index national/cultural identities through language. I then show how they use their linguistic identities to index other identities.

\subsection{Linguistic reflection drawings}

Working with young participants requires careful consideration of appropriate data collection methods. The reliance on interviews or verbal accounts alone is not conducive to accessing the inner psychological aspects of children's identities (Seals, 2017b). For example, during data collection, I asked the students to choose pseudonyms for themselves so that I could use the pseudonyms in my research report. Some of them wondered whether to choose English or Indian names. This led me to pose a follow-up question about how they would identify themselves - a question that brought about contradicting answers to those of the first question. I then asked a third question about which Rugby team they would support if the New Zealand All Blacks played against the Indian national team. Interestingly, some who chose English names and identified themselves as Kiwis reported that they would support India, and vice 
versa. Their responses suggested that they did not seem to have a problem with the lack of consistency in their identification. This highlighted a need for a better understanding of how they understand their 'selves' and the world around them. To this end, I adopt Krumm's (2001) linguistic identity portraits, aka 'linguistic reflection drawings' (Seals, 2017b), which are suitable for young participants to express how they process meaning-making by using language-colour association and using the body as a landscape for the linguistic repertoire (cf. 'embodiment' Bucholtz \& Hall, 2016). The basis of the exercise was the idea that the inclusion of a language in one's linguistic identity is the influence of language ideologies. This way, holding certain language ideologies (whether positive or negative) may not mean excluding that language from one's linguistic repertoire. Rather, what that language indexes to the participants is the goal, allowing accessing to those of relevance to their identities. We need to understand how HL learners make sense of the languages they claim as well as how they evaluate these languages.

Linguistic reflection drawings have been successfully used by researchers as an effective method to use with young participants for exploring language ideologies and linguistic identities. They provide rich data that participants might not able to provide otherwise (Dressler, 2014; Krumm, 2013; Seals, 2017b), and allow for expressions which reflect young participants' linguistic embodied experiences (Gibbs, 2003; Salo \& Dufva, 2017). Additionally, linguistic reflection drawings had another function in this thesis. My young participants were enthusiastic and excited to do the task. One of the students, whose parents asked to opt out of the research, wanted to do the task, so she asked her parents to sign off the consent form to participate. Thus, the task helped contribute to participant retention.

Linguistic reflection drawings begin with "basic, plain, and, if possible, abstract human outlines" (Martin, 2012, p. 41) that enable children to narrate linguistic biographies in a way that illuminates the understanding of "which dispositions and attitudes [...] and which linguistic heritage structures the identity change during the migration process" (Krumm, 2013, p. 165). The task consists of two parts. First, a human outline is given to the participants, one at a time, and they are asked to choose one colour for each of the languages they speak $/ \mathrm{know}$. They are then asked to explain why they associate colours with languages to reveal the associative meanings they assign to their languages. Second, the participants are asked to draw/colour the character outline using the colours they have chosen. Then, they explain the drawing. In this way, linguistic reflection drawings are similar to the introspective methods implemented in applied linguistic research, i.e. 'think aloud' activities (Deschambault, 2017), 
that "help reveal inner psychological aspects of identity construction" (Seals, 2013, p. 158). Thus, linguistic reflection drawings serve as a fruitful task to uncover the inner thoughts of young participants and meaningfully interpret their conceptualisation of 'self', others and languages. The following section includes the presentation of illustrative examples by five learners of Hindi as an HL (Tattu, Ann, Lucy, Havana and Lara) along with an analysis of the descriptive explanations of their drawings.

\subsection{Language as capital: Indexing identity}

The analysis of HLLs' data focused on indexicality in their multimodal descriptions of their linguistic repertoires. The students gave space to multiple languages (two to five languages) in their linguistic repertoires. Many had a '[multi]lingual heritage' (García, 2005) since they spoke English and their heritage languages, including Telugu, Tamil, Kannada, Hindi. Many were learning Hindi in the Hindi School, and some were learning other languages in New Zealand public schools (including Spanish and Te Reo Māori). The data revealed that the indexical meaning that one assigned to a particular language allowed for the inclusion/exclusion of that language in/from one's linguistic identity.

The use of colours created opportunities for delving into indexicalities and language ideologies in the children's discourse. The children used colours as an available mode for the presentational/communicational task (Kress, 2010, p. 27) to communicate their cultural and individualised personas (Kress and van Leeuwen 2001; Salo and Dufva 2017) with reference to language. These indexicalities relied on shared socio-cultural knowledge that the students had as members of the Indian community (in which they were exposed to various aspects of the wider Indian culture) in New Zealand, while also sharing norms of wider New Zealand society.

To understand how HLLs conceptualised the languages they claimed and their language ideologies towards these languages, I analysed the descriptions of their linguistic reflection drawings, with a special focus on the indexical meaning of their languages. The findings revealed that the learners of Hindi as a heritage language in the HS depicted language not only as a tool that served for communicative purposes, but also as a tool associated with different forms of 'capital' (Bourdieu, 1986). This way, the HLLs created semiotic links that were dependent on the interactional context for meaning (Bucholtz \& Hall, 2005; 2010, p. 21; Ochs, 1992). Arguably, this was more the 'direct' indexicality (Ochs, 1993) in their description, but 
in addition, they developed the semiotic links to generate more abstract meanings including the construction of multilingual identities and national/cultural identities.

\subsubsection{Hindi as social capital, HL linguistic capital: Indexing national identity}

The previous chapter indicated that the number of students who regularly attended the classes was around half of the total number enrolled. Parents and teachers also ascribed participation in the school to the perception of the school as a place to gain social capital, by identifying themselves with it and making Indian friends. The learners' linguistic reflection drawings and their descriptions revealed similar trends. In addition to that, the drawings provided rich interpretations of the layers of meaning the learners connected to their languages. In the following example, I demonstrate how Tattu, as an illustrative example of the wider data set, created indexical connections between English and Hindi to build her social capital, reserving Telugu to index her linguistic capital. This allowed her to extend the connections to index her linguistic and national identities as multiple. Tattu is a compelling example because of her explicit and clear expression of thoughts. She was a ten-year-old girl with a Telugu speaking background.

At the beginning of the task, I explained the linguistic reflection drawing process to her. I showed her the body silhouette, saying that I would ask her two questions before colouring it. The task started when I asked 'how many languages do you know?' I chose to use the verb 'know' rather than 'speak' to encourage her to describe her linguistic repertoire as well as to give her more agency in her choice. Then I asked her to choose one colour for each of her languages. Next, she explained her selections before she proceeded to the drawing task, followed by a description of it. The informative responses and turn-taking, in Excerpts 5.1 and 5.2 show her comfort while expressing her thoughts (evidence of the suitability of the linguistic reflection drawings to unearth the inner thoughts of young participants). In Excerpt 5.1 below, Tattu explains her colour choices for the languages she knows.

\section{Excerpt 5.1: Tattu's colour selection (Recorded Interaction)}

\begin{tabular}{cll}
\hline 1 & Moh: & how many languages do you know? \\
2 & Tattu: & I speak three but one of them I just kind of learn \\
3 & & both English and Telugu + two of them and Hindi is one that I'm still learning \\
4 & & but I'm still good at three of them \\
5 & Moh: & what colours would you choose for Telugu? \\
6 & Tattu: & well um Telugu I might choose a dark colour like red \\
7 & & because um it's like really strong language in my family and myself \\
8 & & because my mother and my father and all my relatives + they really + speak it + \\
9 & & it's like our core language \\
10 & Moh: & for English?
\end{tabular}


11 Tattu: and blue for English because when I learnt the first language that-

when I ca- when I came back from India + it was English because I needed to know English to communicate with others + and through it and I was communicating with others and that made me + earn more friendships and + like building relationships Moh: and for Hindi?

Tattu: and for Hindi I might choose green because it's + because even when I'm in India because India (we're) not so familiar with English + so in India it's like opening another door + so then I go into it I see so many other things and then I get more relationships and more friends and etcetera

Tattu claims three languages (line 2), and although she positions herself as a Hindi learner (line 3), she sees no major difference between being a learner or a speaker as she is still good in three of them' (line 4). Tattu explains the connections she makes between colours and languages in terms of their shared qualities. She connects the colour red to Telugu because red is a bold or 'dark' colour (line 6) and Telugu is her 'really strong' language (line 7). Her response shows that she constructs Telugu as linguistic capital. Likewise, she uses labels for Telugu such as 'core language' (line 9) to index her linguistic identity (Bucholtz \& Hall, 2005). Thus, she uses the boldness of the colour red as a direct link to her linguistic capital, which in turn becomes an emblem of her Telugu linguistic identity.

She then connects blue to English because she learnt English in New Zealand (lines 11 and 12), and because she needed English to gain the social reward of speaking English (line 15) that is building social capital by having friends in New Zealand. Similarly, she connects green to Hindi drawing upon its function for building social capital in India. Since she thinks people in India do not speak English (line 18), she perceives Hindi as a resource not only to communicate with people beyond the family borders (line 19), but also to explore the world (line 20). Tattu goes further in connecting English and Hindi as representing New Zealand and India respectively. She leans into the communicative roles of these languages to build her social capital. She also metaphorically conceptualises Hindi as 'opening another door' (line 19) through which she can perceive the world, 'see things' (line 20), build relationships and make friends to establish her belonging (line 21). While Tattu associates Telugu with her linguistic capital, English and Hindi are associated with building social capital due to their functionality in her surroundings. Tattu identifies herself as a multilingual person with Telugu as the main language in her repertoire. While she recognises English as the dominant language in New Zealand, she sees Hindi as a heritage lingua Franca in India. Although Telugu is not a minority language in the south of India, Tattu acknowledges Hindi as a dominant language that is used 
outside of the family settings- a theme that becomes salient when she describes her drawing in Excerpt 5.2.

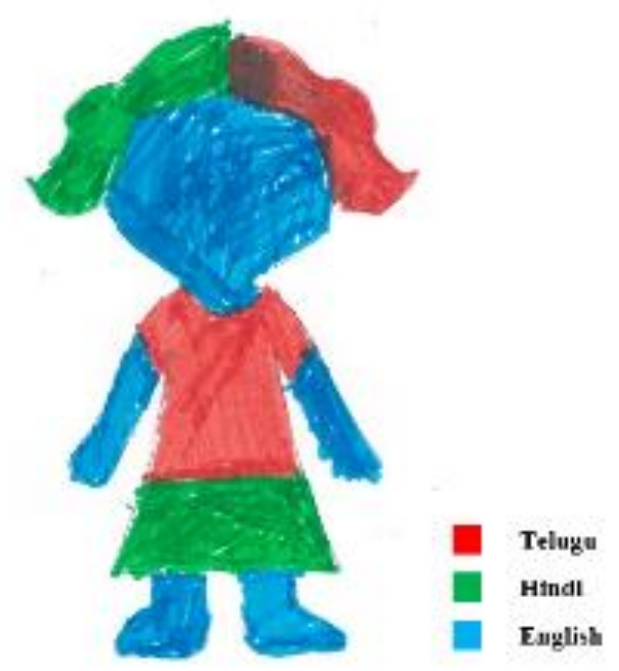

Image 5.1 Tattu's linguistic reflection drawing

\section{Excerpt 5.2: Tattu's description of her drawing (Recorded Interaction)}

\begin{tabular}{|c|c|c|}
\hline 22 & Moh: & tell me what you did? \\
\hline 23 & Tattu: & so I did blue for the arms legs and face because it makes up my body \\
\hline 24 & Moh: & which is English \\
\hline 25 & Tattu: & yeah it's English because I was born here \\
\hline 26 & & and I lived here for a year and I even- I went to kindergarten here + \\
\hline 27 & & so that's + the outside that like I got some more friends \\
\hline 28 & & and I saw many things English + so that's like most English + \\
\hline 29 & & and I chose the green for Hindi and wanted to put it here [the skirt] \\
\hline 30 & & because that's I chose like here's the inside \\
\hline 31 & & because it comes from the inside to the out + \\
\hline 32 & & so like part of me is Indian like half of me is Indian inside + \\
\hline 33 & Moh: & so the top is inside \\
\hline 34 & Tattu: & inside + so the red inside \\
\hline 35 & & and I chose the top as red in Telugu \\
\hline 36 & & because that was like my first language I learnt + \\
\hline 37 & & and first language I noticed that was most related to me + \\
\hline 38 & & and then the green is the skirt \\
\hline 39 & & because + after I discovered Hindi I was really enthusiastic + \\
\hline 40 & & because I learnt that in India it was really hard to communicate with others only \\
\hline 41 & & except my family \\
\hline 42 & & because they mostly spoke Hindi + so I just smile at them and wave with hands \\
\hline 43 & & but I can't say anything else + so that's why Hindi I decided I needed to learn \\
\hline 44 & & and then I (studied it to become) familiar with how I can understand little bit of it + \\
\hline 45 & & and so on the top it's green half of it \\
\hline 46 & & because + like the hair it's like representing I'm Indian+ \\
\hline 47 & & half the two languages that I know is Indian + Hindi and Telugu + \\
\hline 48 & & so then I started to communicate with others very well \\
\hline 49 & & they can only speak Hindi and don't know anything else + \\
\hline 50 & & like my grandma + um she doesn't speak English she doesn't understand it + \\
\hline
\end{tabular}


As shown in Image 5.1, Tattu has coloured her face, arms and legs blue while her hair and clothes are green and red. In Excerpt 5.2, Tattu spells out the reasons why she has created this pattern. Having finished the task, I ask her to describe what she has done. She explains that she colours her extremities blue because English makes up her body (line 23), qualifying this statement by saying that she was born (line 25), lived her early childhood and went to kindergarten in New Zealand (line 26). She describes English as the 'outside' part of her character because she uses it as a resource to gain social capital (line 27) and to perceive the world around her (line 28). Tattu shifts from a linguistic to a national identity displaying herself as New Zealand-born with social capital to indexes her New Zealand national identity.

However, she constructs and negotiates her hybrid identity when she describes the top and the skirt. This description was supplemented by the overt statement in line 32 that 'half of me is Indian', giving her the Indian languages (and thus identity) as the 'inside' half of her character. Tattu displays the cultural capital associated with Telugu and Hindi by deliberately keeping them adjacent as they represent her Indian languages (line 47). The metaphorical placement of the Indian languages together can be understood as an implicature whereby identity construction is indexically processed (Bucholtz \& Hall, 2005). Tattu uses this implicature to index her Indian national identity. When I request confirmation about whether Telugu is included in her Indian 'inside' part, she confirms that it is, giving more details that are crucial to her decision. In her confirmation, she fully claims her Telugu linguistic identity since it is her first language (line 36), but also the 'most related' to her (line 37). Similar to Telugu, Hindi is assigned half of the 'inside'. Tattu qualifies this claim elaborating on the social rewards one can get when speaking Hindi. She believes that, in India, communicating with people other than family members is only possible through Hindi (lines 40 and 41). To Tattu, Hindi is the heritage lingua franca for Indians as people in India speak Hindi and 'do not know anything else' (line 49). Indian identity construction continues when Tattu describes the colours of her hair. Colouring the top of her body and the hair red and green represents the salience of her Indian identity through the two Indian languages (as expressed in line 47). The negotiation of New Zealand and Indian national identities displays Lucy's cultural capital as being multicultural, which arguably reveals her perception of the national identity as multiple.

Another finding in Tattu's description is how she perceives language. Tattu elaborates on the contextual use of her languages in her description of the drawing, touching upon various 
language elements such as comprehension, speaking and vocabulary. In line 53, Tattu refers to Hindi as a 'way to talk' in which she can express herself, and which needs to be understood in a certain way (line 44). If communication cannot be fulfilled using language, other semiotic resources, such as smiling and waving, are used (line 42) but not adequately enough to fully communicate with other people. Language in this sense is meant to create an equal interactional environment for everyone to have a voice (Beres, 2015) where Tattu desires to actively engage in interaction to build her social capital. However, her experience of communicating with other people, e.g. her grandmother who does not speak English (line 50), suggests that her grandmother's usage of 'really huge' and 'big words' in Telugu frustrates her desire to socialize (lines 50 and 51). Thus, this experience has encouraged further investment (Norton \& Toohey, 2001) in another language, Hindi, which her grandmother speaks (and every Indian does according to Tattu) and which does not seem to Tattu to have such 'big words' to overcome. This investment in Hindi may be because Hindi serves as a lingua franca with her grandmother while also being easier for her to manage. Tattu's language ideologies about Telugu and Hindi as Indian languages allow for conceptualising Telugu as having a more complicated vocabulary. For Tattu, Hindi is perceived as easier to use to fulfil her communicative goals, whether within the family or beyond.

The Hindi-dominant language ideologies were clear in Tattu's discourse. She invested in Hindi because, while in India, she had the desire to communicate with 'others' who, in her views, 'can only speak Hindi and don't know anything else' (lines 49). This connection could stem from a nationalist ideology in India since 1930 that led to "a categorization of Hindi as the mother tongue or matra bhasha" (Groff, 2018, p. 17). This view reflected a language ideology that Hindi was the dominant and national language in India, a notion that was highly emphasised in the HS 'Big-D' Discourse. Such a nationalist ideology was supported in the Hindi School's domain (See section 4.2), community (See section 4.3) and shared discourse (See section 4.4.3). Tattu's linguistic reflection drawing also showed that she included the three languages in her drawing to emphasise the importance of these languages to her life socially, historically and emotionally. While Telugu was her 'core language' and the 'most related' to her, English and Hindi were of high significance. Despite her evaluative statement of her knowledge in Hindi as a learner, she viewed Hindi as necessary as Telugu was to her Indian identity. 


\subsubsection{Hindi as cultural capital: Indexing cultural identity}

In addition to using labels and (evaluative) stance-taking, the negotiation of the linguistic and cultural identities is indexically connected to language through positioning and the use of certain features of discourse. An example of this is presented by Ann. Ann provides a prime example of how members of the HS CofP are influenced by their membership in the HS by using the broad definition of 'mother tongue' that is dominant in the HS Discourse whereby Hindi is conceived as the language used by Indians. At the time, Ann was a nine-year-old girl from a multilingual family. She was older than her sister, Mylu. Ann's father spoke English natively. He also spoke his heritage language, Gujarati. Her mother, Kaya ji, spoke (Fijian and Shuddh) Hindi and English. Being born and raised in New Zealand and having English as the language of communication among family members did not prevent Ann from giving Hindi a place in her linguistic identity. Like Tattu, Ann provides a lot of detail when answering questions as shown in Excerpt 5.3.

Excerpt 5.3: Ann's colour selection (Recorded Interaction)

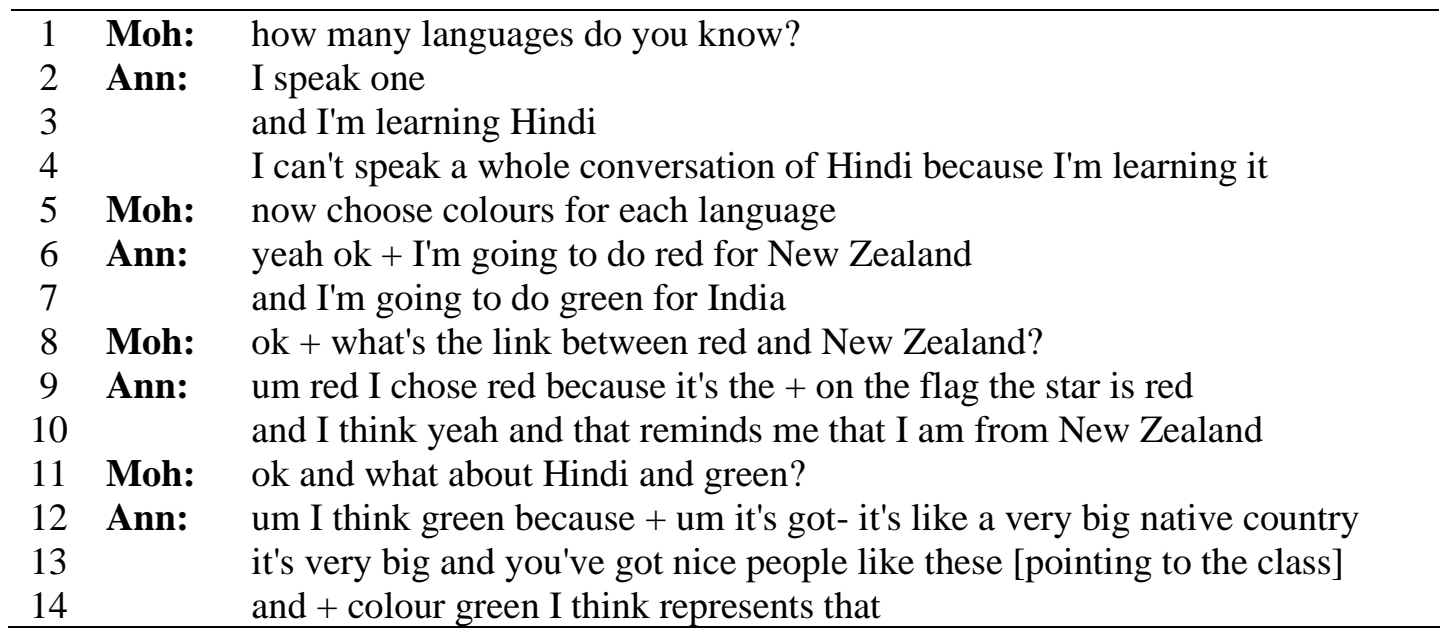

When asked how many languages she knows (line 1), Ann offers more insights than the question requires - a point that supports the usefulness of the linguistic reflection drawings. In her response, she fully claims her linguistic identity as a speaker of English. Further, she categorically identifies herself as a 'learner' of Hindi rather than a 'speaker' (line 2). Ann contributes more to the negotiation of her Hindi linguistic identity through the evaluative comment on her inability to engage in a full conversation in Hindi (line 3), ascribing it to her status as a learner (line 4). In line 5, I ask her to choose colours that represent her languages. Interestingly, she associates red and green with New Zealand and India respectively (lines 6 and 7). The way Ann expresses these associations suggests that she sees that English represents New Zealand and Hindi represents India, which in turn reveals her understanding of the 
dominance of these languages in the given societies. I follow up in line 8 by asking her to explain the association she has made between red and New Zealand. At this point, she attributes this assignment to the red stars in the national flag of New Zealand (line 9) which stands as a reminder of her national identity as a New Zealander (line 10), the national flag as a sign of nationalism which is indexically tied to identity (Bucholtz \& Hall, 2005).

However, she shifts to another theme for associating green with Hindi. Assuming that she has accidentally conflated languages with countries, I ask her in line 11 to explain why she has chosen green for Hindi (but not India). Her response confirms the deliberate extension of the connection from colour-to-language to colour-to-country. She directly connects green to India's attributes by describing it as 'a very big and native country' (line 12) that has got 'nice people like these' referring to the Hindi class (line 13). The reference she made to the HS members in line 13 by saying 'like these' calls attention to her experience with Indian people in the HS. Interestingly, she uses New Zealand discourse, e.g. the word 'native', to refer to India's green and agricultural nature in line 12 by saying 'it's like a native country'. Building upon Bucholtz and Hall's (2005, p. 594) indexicality principle which suggests that identity is indexed through "the use of linguistic structures and systems that are ideologically associated with specific personas and groups", Ann's use of the word 'native' indexes her internalisation of the New Zealand discursive norms which in turn indexes her identity as a New Zealander. Yet, she uses it to foreground her newly experienced Indian world during her trip to India (of which I learnt in the interview with Ann's mother) as well as her experience in the HS (line 13). The different connections Ann describes for her languages suggest that while she connects red to the flag of New Zealand, indexing her strong sense of belonging to New Zealand, she balances this identity by connecting green to India, indexing her recent experience with India and the HS. 


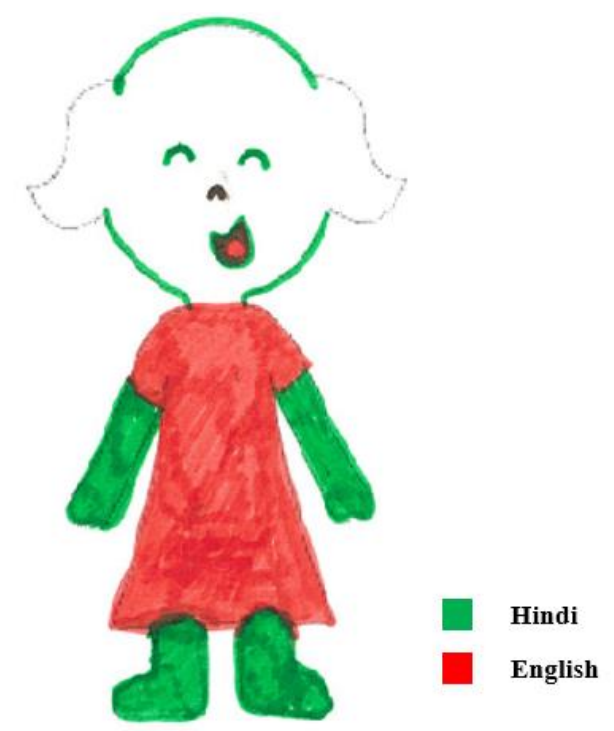

Image 5.2 Ann's linguistic reflection drawing

Having chosen colours and explained her choices, Ann proceeded to reflect on what she would do before colouring her drawing. Prior to describing her drawing, I anticipated that she would follow the dyadic pattern of inside and outside that emerged in the drawings of other participants. Yet, the description in Excerpt 5.4 shows that she does the colouring to reflect on the New Zealand lifestyle she leads. As shown in Image 5.2, Ann colours her 'dress' red and her extremities green. She adds some details to the face including the eyes, nose and mouth to allow more space for language functions. Excerpt 5.4 below outlines Ann's reflexive strategy while drawing, where she holds a colouring pen, thinks aloud and then colours the relevant parts of the drawing.

Excerpt 5.4: Ann describes her linguistic reflection drawing (Recorded Interaction)

15 Moh: you'll colour yourself as you see yourself with these two languages [Ann holds the red colouring pen]

Ann: well + I feel like my: body here [the dress] would be New Zealand + because + I'm here and my dress will be New Zealand + with the colour that represents New Zealand because $+\mathrm{I}$ kind- I'm born here and I think + uh [smile] that + you know + I'm wearing where I came from + I'm wearing what I am who I am + I'm a New Zealander. so I think my clothes would be ++ would be [started colouring] red + I'm just going to draw that in. [Ann starts drawing] [Ann holds the green colouring pen] and my skin, tongue an:d my mother tongue would be my body so my skin yes

Moh: so + is Hindi your mother tongue?

Ann: yes my mother tongue + Hindi is my mother tongue and I was + I'm growing up here in New Zealand + this is my home and I'm doing my body green because my mother tongue is it.

Moh: what language did you speak when you were kid? 
Ann: um English English +

my mom and dad used to put little Hindi words in there

like + um we went to meet someone in our family or something

we'd say 'namaste' each time we meet or like 'chalo' like 'hurry up' + and stuff like that

but I + I usually speak English + because I use it at school.

[Ann resumes drawing]

Moh: tell me what you did here [the mouth]

Ann: well what I did is + on the mouth I did red

because I mostly speak English +

but I've got a little bit of green there

Moh: and why did you have a little bit of green?

Ann: um because + well sometimes at home we practice it +

and then + in the Hindi class we prac- I + I speak it + I like kind of speak it +

so + I sometimes speak Hindi + so I put a little there ++

and when my grandmom and dad come over um +

they speak little bit of Gujarati to me + and I- they speak Gujarati

Moh: and what about the nose and the eyes?

Ann: um well for the nose + um + I think for the nose+ it's- + well we eat Indian food

so I put a little green and I put a little red in there +

we eat Indian food most of the time and

I like to smell the flavour (of the cooking) + so that's the nose + and the eyes are green because I see lots of Indian people around + my family and some of my friends

Ann begins by expressing what she wants her drawing to look like, describing her English part of the drawing. She notes that her dress represents New Zealand (line 16). She further explains her choice in detail by listing her present life in New Zealand (line 17), her place of birth (line 19), and lifestyle (20) to construct her New Zealand national identity. Ann supplements her identity construction with clear-cut identification including historicity 'where I came from' (line 20), inner identification 'who I am' ending up with full and overt ownership of the New Zealand national identity (line 21).

Similarly, she spells out her thoughts regarding the Hindi part of the drawing before she commences colouring it. She explains that she wants to assign green to her body because Hindi is her 'mother tongue' (line 24). Notably, while she positions herself as a Hindi learner, she expresses a strong claim to the Hindi linguistic identity (lines 24, 26 and 28). This claim drives me to ask what language she spoke in her early childhood (line 29), a question to which Ann responds in line 30 by stating that she spoke English in her early childhood. Seemingly, Ann is inclined to negotiate her Hindi linguistic identity when responding to my question. She qualifies her claim to the Hindi linguistic identity by referring to her early exposure to Hindi through translanguaging practices (lines 31 through 33). In line 31, she foregrounds a wellestablished relationship with Hindi as she has been exposed to Hindi since she was a child. To her, using bits of language with members of the extended family (line 32) qualifies her to claim 
Hindi as a mother tongue. Interestingly, such understanding reflects the broad definition of the term "mother tongue' that is used in the HS to refer to HLs and Hindi (section 4.2). Thus, although she does not claim an Indian cultural identity, she uses Hindi linguistic capital as a mother tongue to index it.

Ann resumes drawing right after she has a mental visualisation of what details her drawing will include. In line 36, I request a further explanation of the details she does to the face (the mouth). She explains that she considers the frequency of language use to colour the mouth where it is 'mostly' English (line 38) with 'a little bit' of Hindi (line 39). When I inquire about why she places 'a little bit' of Hindi, she mitigates her response by pausing and hedging words such as 'sometimes' and 'kind of' (lines 41 through 43). Yet, she retains her claim to Hindi as she feels it is alive in the home and the HS, acknowledging her awareness of her Hindi learner identity. Remarkably, Ann introduces Gujarati in line 45 as a continuation of the negotiation of her Indian cultural identity. She integrates being talked to in Gujarati and having Gujarati-speaking grandparents into the negotiation of Hindi as a mother tongue. Despite mentioning being socialised in Gujarati, Ann does not claim Gujarati as one of her languages. This might be because her mother, Kaya ji, does not speak Gujarati and she is the one who is responsible for 'language stuff' at home (as Kaya ji stated in the interview). There is also a possibility that this is due to the influence of the HS on her perception of the status of Hindi as the mother tongue of all Indians mother tongue. Ann continues explaining the nose and the eyes by recalling 'bits of culture' (Blommaert, 2010). She draws upon her receptive senses smelling and seeing of Indian food (lines 47, 49 and 50) and socialising with Indians in her surroundings (line 52) to colour the nose and the eyes. Thus, Ann associates Hindi with the cultural capital she displays by foregrounding the 'bits of culture' she likes as well as social capital by projecting her Indian surroundings within the family and the HS.

Ann's association of languages with colours suggested that Ann's language ideologies towards English and Hindi were positive. It also indicated that these language ideologies derived from different resources such as family, the HS and life experiences. Ann used her English as a linguistic capital to index her New Zealand national identity, and although she used the colour green to index India through the language ideologies she held regarding Hindi, she negotiated her Hindi linguistic identity as a result of the cultural and social forms of capital associated to it, which in turn indexed her 'Indianness' or Indian cultural identity. Overall, Ann's negotiation of her linguistic identity as multilingual led her to negotiate her cultural identity as multiple 
where she displayed belonging to both New Zealand wider society and Indian wider society, accessed through the HS.

\subsubsection{HL as cultural capital: Indexing cultural and national identities}

Another theme emerged in the discourse of some participants, especially those who did not include Hindi in their linguistic repertoires. These participants conceptualised the HL as a particular resource for displaying cultural capital. They then indexed their linguistic, national and cultural identities. An example of this case was Lucy, who was an eleven-year-old girl with a Tamil background. She was born in the UK and came to New Zealand in her early childhood. She had been to India several times. Lucy joined the HS towards the end of the school term (the last two weeks of the observation phase) which could be a reason why she excluded Hindi from her drawing. In Excerpt 5.5 Lucy chooses colours and explains her choices.

Excerpt 5.5: Lucy connects colours to languages (Recorded Interaction)

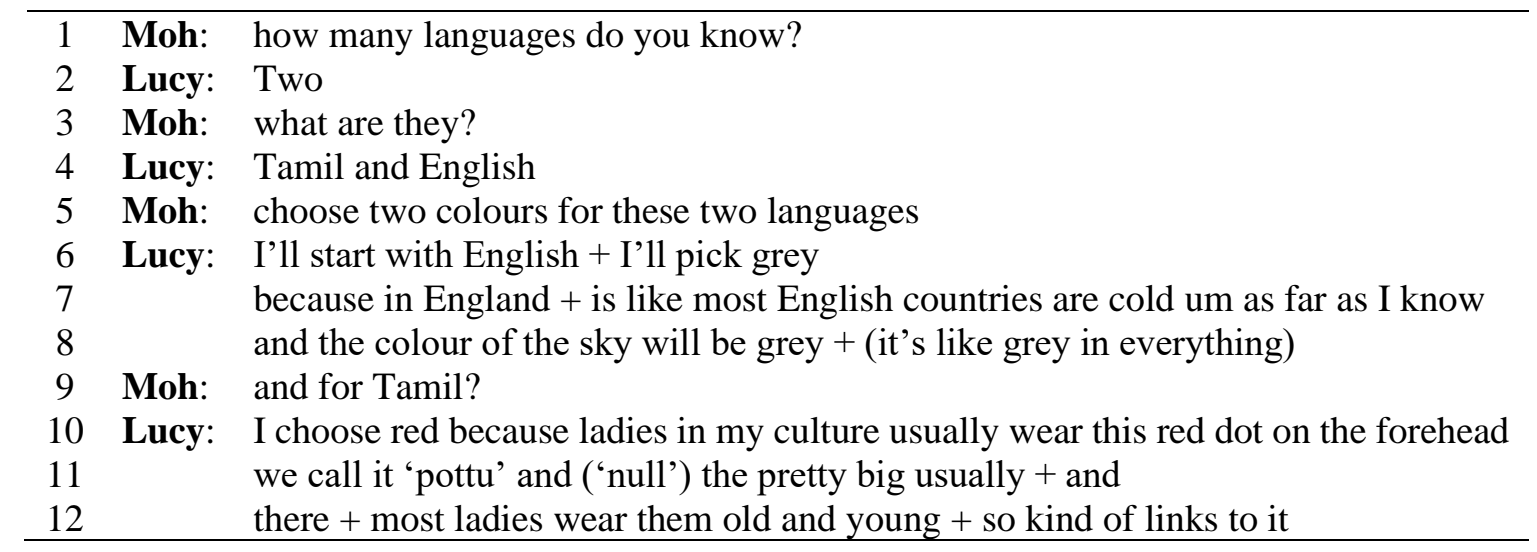

As noted in Excerpt 5.5, Lucy reports knowing Tamil and English (line 4). She connects grey to English drawing upon her life experience in England. Thus, she connects English with the cold weather and cloudy sky of most English-speaking countries (line 7 and 8). In contrast, she connects red to Tamil through the red mark (Tilak) that is worn on the forehead as an ornament in India. Doing so, she refers to two levels of her Indian identity. She first refers to her own Tamil culture (line 10) although the Tilak is a Hindu religious sign. Noting that while Lucy connects English with how she perceives English speaking countries, she connects Tamil with the Tilak as a unique mark of her Tamil cultural identity. This might suggest that emotional (internal) links go with Tamil while physical (external) links go with what she has now in hand (the reality that she lives here and now). Lucy uses the first-person possessive adjective ' $m y$ culture' (line 10) and the first-person pronoun 'we call it' (line 11) to display her legitimate membership of the Tamil culture (and perhaps to exclude me). She also shows her knowledge 
of the culture as she proudly and readily teaches her interlocutor who wears the Tilak, where it is worn, the subtle differences between its kinds and its Tamil names (lines 10 through 12). Taking such an epistemic stance of profound knowledge of the culture indexes her belonging to the Tamil culture as cultural capital, giving more salience to her Tamil cultural identity than to her linguistic identity. Second, she refers to her Indian national identity in her linguistic reflection drawing (Image 5.3) and the description she provides in Excerpt 5.6.

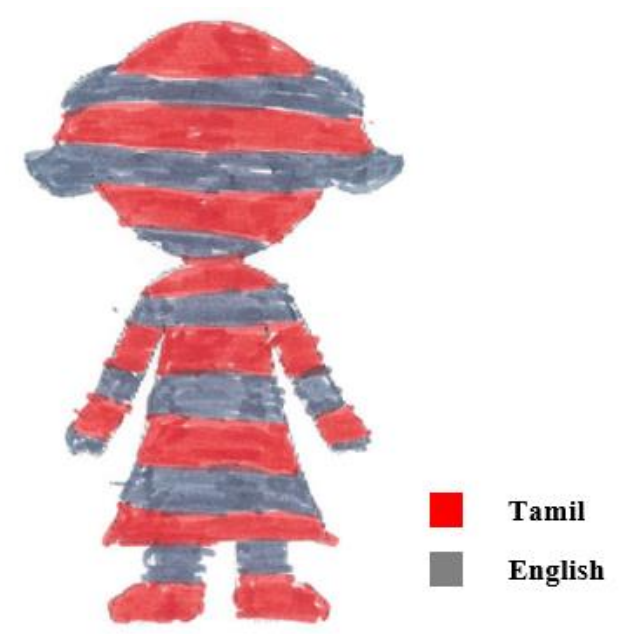

Image 5.3 Lucy's linguistic reflection drawing

Excerpt 5.6: Lucy's description of her drawing (Recorded Interaction)

\begin{tabular}{lll}
\hline 13 & Moh: & what did you do? \\
14 & Lucy: & so I coloured it in stripes + \\
15 & & em because India has mainly stripes in its um + it's + um flag \\
16 & & while it does have (a blue) circle I don't know how to make it \\
17 & & and while I live in Wellington, I don't have any family here \\
18 & & my family's all in India + so if I have ( ) like help (to find) things \\
19 & & I go back to um India because family + \\
20 & & I mean I have friends here but they they don't- they can help (but not) so much \\
21 & & so I feel like I should I wanted to base it on India the colouring + so that's why \\
22 & Moh: & which part is bigger the red or the grey? \\
23 & Lucy: & they're both equal because I coloured them in stripes \\
24 & Moh: & does this represent any concept to you + any idea? \\
25 & Lucy & yeah it's supposed to represent the Indian flag which is where I am from + \\
26 & & yeah and um because I'm living in New Zealand \\
27 & & and I have friends here + \\
28 & & I'm studying in New Zealand + \\
29 & & um I decided to have the grey as well \\
\hline
\end{tabular}

As shown in Image 5.3, Lucy fills her character outline with red and grey stripes, explaining her inclination to simulate the pattern of the flag of India (lines 15 and 25). Her desire to draw the Indian flag is hindered by her inability to draw the 24-spoke wheel at the centre of the flag (line 16); a view that reflects how much she is attached to her Indian national identity (again 
the flag representing national identity as in Excerpt 5.3. Lucy extends her explanation shifting from Indian nationalism to the social component of her Indian roots (line 17). Thus, she recognises her social capital in India, represented by family (lines 17 through 19), without denying her social capital in New Zealand (line 20). In doing so, she pragmatically uses both forms of social capital to construct her multilingual identity and then uses these resources as the motive to express the urgency and necessity of indexing her Indianness (lines 21). When I ask Lucy to explain whether one language is given more space in her drawing, she shows loyalty to both as they are equal (line 23). When I ask her if the drawing has some meaning to her, she emphasises her Indian national identity as she desires to draw the 'Indian flag' which represents where she is from (line 25). Yet, because of friends and schooling (i.e. the social and cultural capitals she has in New Zealand (lines 26 and 27)), she acknowledges the 'here' and 'now' and includes grey in her character.

Lucy's linguistic reflection drawing and its description showed that Lucy did not include Hindi in her linguistic identity which could be due to her late enrolment in the HS. Additionally, while Lucy acknowledged her 'route' to New Zealand, she did not refer to a New Zealand national identity. To her, the equal status that Tamil and English had represents the Indian flag which in turn indexed her 'root' Indian identity. She demonstrated how she constantly negotiated being an Indian in New Zealand who had social and cultural capitals associated with both languages, Telugu and English. While she displayed a strong attachment to her Indian identity, the presence of English-related (and potentially New Zealand) social and cultural capitals supported the inclusion of English in her drawing. Now I turn to another aspect of the indirect indices that emerged in the data, namely using one form of identity to index another.

\subsection{Identity as capital}

While the previous examples illustrate indexing and negotiating a repertoire of identities primarily related to English and Indian languages, the following examples shed light on the inclusion of other languages such as Mandarin, Cantonese and Spanish. This sense of diversity and celebration of multilingualism and multiculturalism not only reflected the dominant Discourse in the HS, but also offered examples of how HL learners use one identity to index another. In this section, I demonstrate how HLLs (after using language as capital) extended the indexical ties between their languages and the previously constructed identities to negotiate other identities. First, I present Havana's linguistic reflection drawing and the accompanied description of it. I then present Lara's drawing. 


\subsubsection{Multilingualism and multiculturalism as a capital: Indexing the Kiwi identity}

Havana was the oldest student in the HS (17 years old). She came from a family who had a repertoire of multiple languages including Fijian Hindi, Mandarin, Cantonese and English. Havana and her siblings spoke English as a first language. Her father spoke (Fijian and Shuddh) Hindi and English while her mother spoke Mandarin, Cantonese (which Havana referred to as Chinese) and English. English was the language of communication among family members. As shown in Excerpt 5.7, it was not an easy task for Havana to 'confess her mind'. She wanted the drawing to reflect not only her linguistic identity, but also to represent her multicultural heritage. Havana's discourse is replete with mitigation, pauses and the use of the filler 'like', perhaps to give herself time to process a more abstract representation of her linguistic repertoire as well as to negotiate it (especially if we consider this use of 'like' to index young female identity in New Zealand (Drager, 2015))

\section{Excerpt 5.7: Havana connects colours to languages (Recorded Interaction)}

\begin{tabular}{|c|c|c|}
\hline 1 & Moh: & how many languages do you know? \\
\hline 2 & Havana: & I speak + three \\
\hline 3 & Moh: & what are they? \\
\hline 4 & Havana: & Chinese English and Hindi + that's three \\
\hline 5 & Moh: & would you choose three colours for these three languages? \\
\hline 6 & Havana: & ok [choosing colours] \\
\hline 7 & Moh: & which for which? \\
\hline 8 & Havana: & so red is Chinese and green is for English and orange is for Hindi \\
\hline 9 & Moh: & um why is red for Chinese? \\
\hline 10 & Havana: & um because red is a significant colour in China \\
\hline 11 & & like they say good luck kind of + \\
\hline 12 & & one as well as it's like + the most colour that they associate with \\
\hline 13 & Moh: & and you? I mean why do you link red to Chinese? \\
\hline 14 & Havana: & well + the link to the flag and the stars as well as \\
\hline 15 & & it's like a sign of like + in the Chinese culture is like sign of happiness as well \\
\hline 16 & & so it's kind of like a very- significant colour in the culture and everything + \\
\hline 17 & & so they use red in all their traditions and everything like that + yeah \\
\hline 18 & Moh: & and why is green for English? \\
\hline 19 & Havana: & to me + um ++ well [pausing 7 seconds] I don't know why \\
\hline 20 & & but I feel like when I think of English I think of New Zealand and \\
\hline 21 & & New Zealand is like green + it's like (a lot of green) and stuff \\
\hline 22 & & so that's how I associate + with + green + with like + English + sort of + yeah \\
\hline 23 & & it's kind of hard to explain \\
\hline 24 & & but it's just I feel like when I think of English I think of green and blue mostly \\
\hline 25 & Moh: & why do you think of green and blue when you think of English? \\
\hline 26 & & is there any link or something that reminds you of these colours? \\
\hline
\end{tabular}


Havana: not really it's just a colour I link like it (to confess my mind) what I thought of mostly like + so like in New Zealand and then like + I don't know like + the ferns kind of + but also the first thing um kind of came like symbolic as well + because like it's like + I don't know it's like + native + yeah + nature yeah like + I see the fields + that's why I picked green + but I was thinking of blue because of the flag as well but you know I wanted some different as well + because it's not just blue + there's more than just blue + like there's other aspect to New Zealand and other culture as well + like it's not just one colour + because the community is represented in many ways + yeah

Moh: $\quad$ and orange with Hindi?

Havana: orange is also part of the flag [laugh] as well + but also + don't know + the food and other spices? [laugh]

Havana claims three languages, Chinese (i.e. Mandarin and/or Cantonese), English and Hindi (lines 2 and 4) for which she chooses red, green and orange respectively (line 8). When asked to explain her colour-language associations, she connects 'red' to Chinese through bits of the Chinese culture (lines 10-12 and 15-17) and refers to it as part of the national flag of China (line 14). Havana explains her choice of red by taking an epistemic stance of knowing the Chinese culture, noting the significance of the colour red in the Chinese culture (line 10), and that red indexes 'good luck' and 'happiness' (lines 11 and 15). Havana uses this stance to index her Chinese cultural identity as part of her maternal heritage.

Havana also connects green with English because of the mental association she has between English and New Zealand (line 20). This connection foregrounds New Zealand's 'native' agricultural nature and multicultural diversity. In the beginning, Havana seems to oscillate between blue and green for English. The long pause, reporting not knowing (line 19) and the explicit speech of her mind 'when I think of English I think of green and blue mostly' (line 24) suggest this alternation. While she connects blue with English because of the predominantly blue flag of New Zealand (line 33), she opts to choose green for English since New Zealand is a multilingual, multicultural country (lines 36 and 38). She qualifies her choice by addressing the interviewer's epistemic stance. Her 'you know' (line 34) evokes shared sociocultural knowledge that the interlocutor or the vague 'you' is expected to know about the country. Furthermore, Havana explicitly addresses the shared knowledge and supports her choice in line 35 by saying 'there's more than just blue' to index contesting the 'one nation-one-language' ideology. Rather she presents New Zealand as represented by its natural and cultural attributes (line 36). Havana's selection of green for English can be understood as using New Zealand discursive features which in turn index constructing her New Zealand national identity which 
she reveals explicitly in Image 5.4 where she refers to her Kiwi-born heart as 'iconic'. Most importantly, the detailed metacognitive account for explaining her connections gives salience to how she wants to be seen. That is, since she wishes to foreground her English (and thus Kiwi) identity, she performs the New Zealander identity in her description by showing the epistemic stance of knowing various aspects of New Zealand culture, and acknowledging multiculturalism, multilingualism as well as nativeness of the country. Thus, Havana uses her languages (and thus her linguistic capital) as a source to index the symbolic capital she has as a multilingual and multicultural person, which in turn qualifies her as belonging to multilingual and multicultural New Zealand.

Havana slightly shifts her connection when she links orange to Hindi. She explains that orange is part of the national flag of India (line 40). Again, she shows oscillation in line 41 by saying 'don't know' before she uptalks (Warren, 2016) in the additional connection with aspects of the Indian food and spices, accompanied by laughter. The laughter suggests that she seems unconvinced by those connections and they might not be suitable for her age or for qualifying such a connection. Interestingly, Havana and her brothers connect different colours with Hindi, but use the same connections (i.e. the flag and food). This could be the result of having this kind (and only this kind) of access to the Indian culture. She uses these resources to index her Indian heritage, and although she displays shades of her Indianness, she does not overtly claim the Indian national identity.

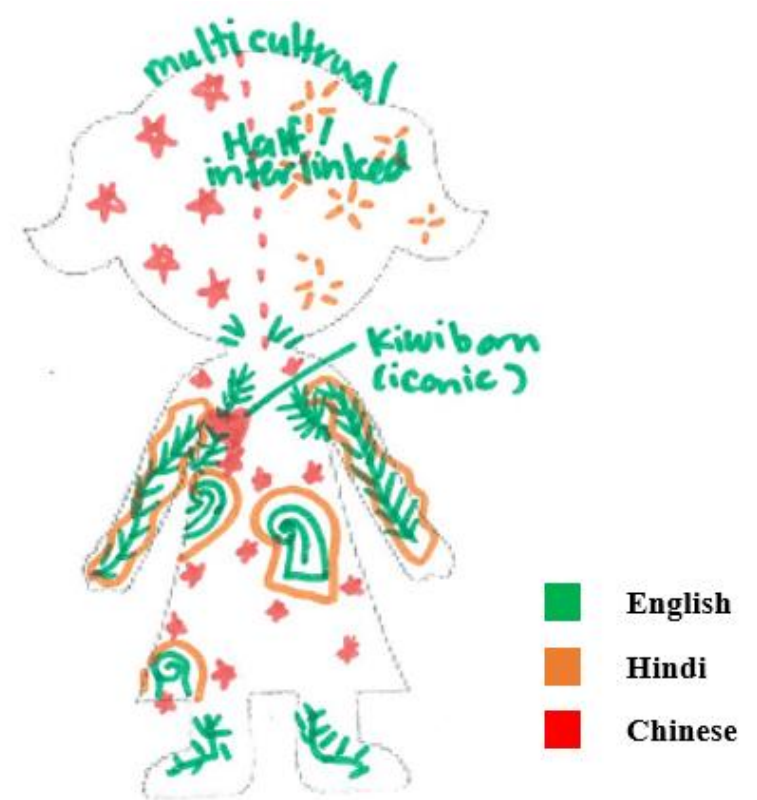

Image 5.4 Havana's linguistic reflection drawing 
As shown in Image 5.4, Havana fills her character silhouette with symbols representing the three cultures to show her intertwined multilingual and multicultural identity. While the green ferns and korus represent her English and 'Kiwi-born' identities, the quintet red stars, which are similar to the ones in the flag of China, represent her Chinese heritage. She also includes orange Indian-ornament-like stars to represent her Indian heritage. She splits the head vertically between Chinses and Hindi with a comment to note the equal and interlinked relationship between the two. The image also shows the word 'multicultural' on the top which she claims indexes English as the 'main' and 'stable' language as noted in Excerpt 5.8.

Excerpt 5.8: Havana describes her linguistic reflection drawing (Recorded Interaction)

\begin{tabular}{lll}
\hline 42 & Moh: & ok what did you do? \\
43 & Havana: & so um + at heart + is like since we're Kiwi born + \\
44 & & and then half and half + so half Chinese half Indian + \\
45 & & it's like in the mind as well like everything all interlinked together + \\
46 & & and it's like bringing everything together multicultural + \\
47 & & and I put some like um + symbolic stuff like + \\
48 & & the fern like the nature of New Zealand as well \\
49 & & and everything kind of combining together into one. \\
50 & Moh: & is there any part larger than the other? \\
51 & Havana: & I think that all kind of equal \\
52 & Moh: & what is the most used colour in it? \\
53 & Havana: & probably red but there is more green here [the face] and extra writing \\
54 & Moh: & does this English writing have any extra meaning? \\
55 & Havana: & well I can think of it + I guess since it is green it's English + \\
56 & & it's like English is my + main language that I use \\
57 & & like it's + like it's just like my stable language that I use \\
58 & & and then Chinese and Hindi are + they are also there + \\
59 & & they're both kind of strong \\
60 & & but English is my main + one I feel most comfortable with + \\
61 & & and communicate with friends and others with + \\
62 & & but I still use Chinese and Hindi + yeah \\
\hline
\end{tabular}

In Excerpt 5.8 Havana explains her linguistic reflection drawing. She starts with the representation of the heart which anchors her New Zealand national identity since she is 'Kiwiborn' (line 43). She then proceeds to the head displaying her cultural capital as a multicultural 'half-Chinese-half-Indian' person (lines 45 and 46). After that, she offers a general description of the rest of the body, emphasising the intertwined relationships among her languages. When I ask her whether the drawing includes dominance of one over the others, she expresses the desire to have them equally represented (line 51). Nevertheless, when I recast the question, she mitigates her response by saying 'probably red' and explains that there is "more green" on the face (line 53). In lines 55 through 62, Havana explains the meaning of the written comments in the drawing. While doing so, she negotiates her multilingual identity by displaying linguistic 
capital. For example, she says that English is her 'main' (lines 56 and 60) and 'stable' language (line 57). She also feels that English is her most comfortable language (line 60) and she socialises through it. In so doing, Havana fully claims a linguistic identity as an English speaker. Simultaneously, she negotiates her multilingual identity by claiming ownership of Chinese and Hindi, supporting this claim in lines 58, 59 and 62 by saying 'they are also there', 'they're both kind of strong', and 'I still use Chinese and Hindi'.

Havana's linguistic reflection drawing along with the description revealed that she took an epistemic stance of knowing her Kiwi, Indian and Chinese heritage cultures to display the cultural capital associated with them. She also used her multicultural heritage (i.e. cultural capital) as a resource to qualify claiming Hindi and Chinese, which in turn indexed her linguistic identity as multilingual. She simultaneously used her linguistic and cultural capitals to index her New Zealand national identity since she was, like New Zealand, a multilingual and multicultural person. Havana conceptualised her languages in a quite similar orientation to Lara who produced the drawing I describe next. However, it is interesting to see how Lara reversed the indexical process and made use of the languages she spoke and was learning.

\subsubsection{Learner linguistic identity: Indexing the Indian cultural identities}

Lara was a twelve-year-old girl with a Telugu speaking background. She was highly invested in learning Hindi and was positioned by her teacher as a leading learner in the HS. Unlike Havana who positioned herself as a Hindi speaker, Lara aligned her self-positioning with the way she was positioned in the HS, i.e. as a Hindi learner. She also included Spanish in her drawing and positioned herself as a learner of Spanish. Interestingly, her drawing and its description revealed the rationale behind her choice. As shown in Excerpt 5.9, Lara reports knowledge of four languages; Telugu, Hindi, English and Spanish (line 4). She connects bright green to Hindi (line 8) because it is a bright and new language which she is learning (lines 10 and 11). She also connects blue to English because she knows it (line 15) and because she thinks that people usually use blue to index English (lines 16 and 17). Then she explains that people may connect blue to English because the flag of New Zealand is mainly blue (line 20). In this way, Lara recruits other people's shared knowledge and echoes their experiences in her explanation. The 'doubled-voice discourse' (Bakhtin, 1994) in Lara's explanation serves to validate what she sees as a 'widely known' connection between blue and English. 
Excerpt 5.9: Lara connects colours to languages (Recorded Interaction)

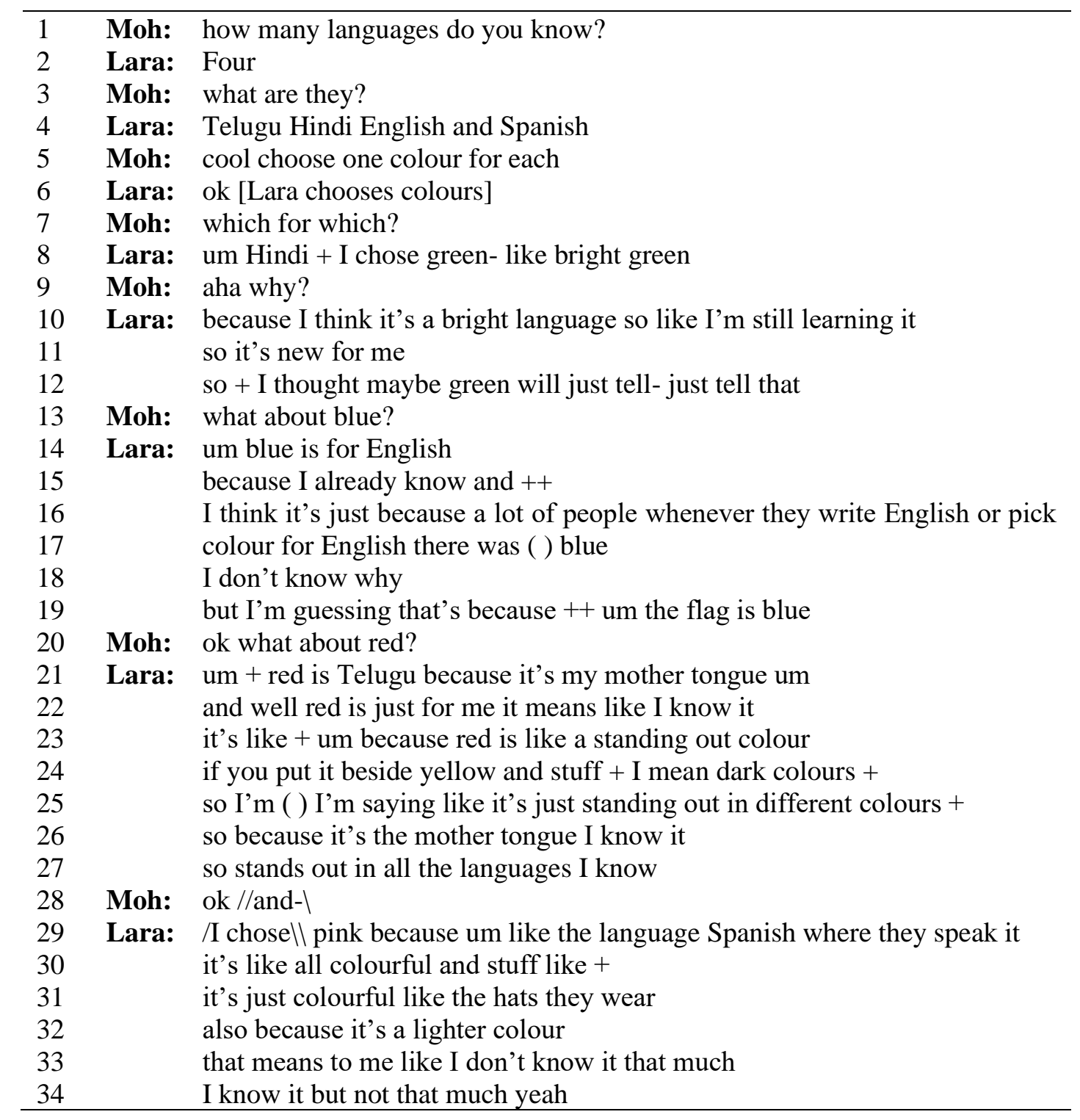

Lara connects red to Telugu because she perceives both the colour red and Telugu as 'standing out' from the rest of the colours and languages (lines 21 and 27). Similar to other learners, Lara overtly constructs her linguistic identity in line 21 by the explicit introduction of the identity label 'mother tongue' to refer to Telugu. For Spanish, she chooses pink because sees it as a colourful language (lines 29-31). She also explains that pink is lighter than red and blue, which means that she is still learning the language. Thus, Lara uses bright and light colours to index her linguistic identities as Hindi and Spanish learner. Likewise, Lara positions herself as a Hindi learner rather than a speaker despite the positive language ideologies towards the language (line 10). Again, she displays her ownership of Telugu as it is her 'mother tongue'- a label that is used three times to refer to Telugu (line 21, 26 and Excerpt 5.10 line 47). she proposes Telugu as linguistic capital which is connected to her linguistic identity as a speaker 
of Telugu. Lara creates space for each of the languages she speaks and is learning to be part of her identity to refer to her linguistic identity as multilingual, which in turn qualifies her to fit in the wider communities she belongs to. Such an interpretation is supported by her drawing as shown in Image 5.5 and its description in Excerpt 5.8.

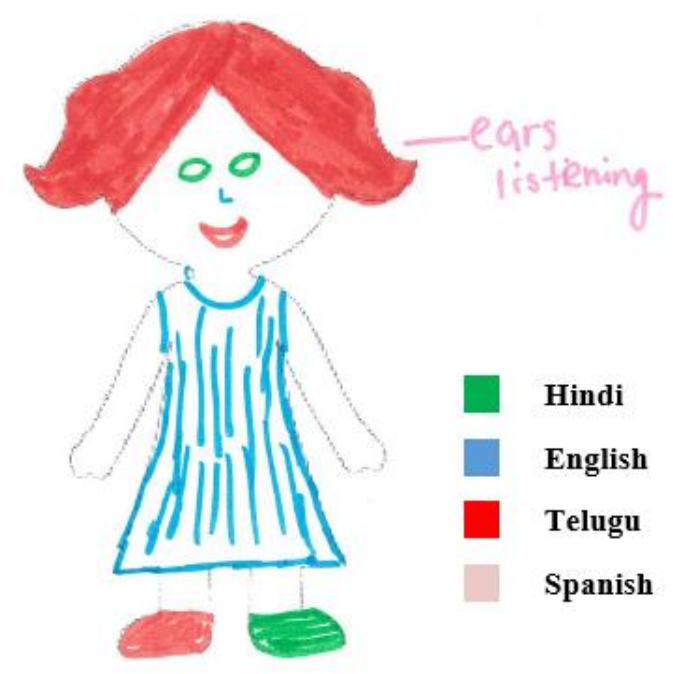

Image 5.5 Lara's linguistic reflection drawing

Lara's linguistic reflection drawing illuminates the understanding of Lara's identity negotiation. Notably, she places Telugu, Hindi and English inside her character whereas Spanish appears in a comment to indicate that she only listens to Spanish. Each part of her character is symbolically filled with what she sees as relevant to her contextual use of language as expressed while explaining the drawing. She places Telugu on the hair, the mouth and one shoe while English is used to draw the dress and nose. She also draws the eyes as Hindi. Like Tattu, Lara indexes her Indian national identity through the implicature of placing Hindi adjacent to Telugu when she draws the shoes. Although the drawing is open to a range of interpretations, the explanation that Lara offers indicates that she associates each of her languages with its respective capital to display multiple identities.

Excerpt 5.10: Lara describes her linguistic reflection drawing (Recorded Interaction)

\begin{tabular}{lll}
\hline 35 & Moh: & tell me what you did \\
36 & Lara: & um the blue is for the dress because it's just long \\
37 & Moh: & what do you mean by 'long'? \\
38 & Lara: & that's + like + um it's a big part \\
39 & & I think + that's because I need speaking that a lot like wherever I go outside \\
40 & & English is the first thing that comes out of the mouth \\
41 & & um and I chose red for the hair \\
42 & & because + um well that's what I remember \\
43 & & I mean that's the part where I remember like \\
44 & & because + yeah the brain
\end{tabular}


Moh: yeah yeah sure you can

[Lara adds details to the face and colours the shoes]

54 Moh: so what about your mouth?

55 Lara: I chose Telugu for my mouth I did red for my mouth

um because that's where I (collect in) the brain and that's where I will remember Telugu because it's my mother language so I always remember it and then I chose pink for the ears because um I'm still listening to Spanish because I'm just learning it so I need to listen to my teachers and stuff to learn Spanish so (by listening) and green [the eyes] for Hindi because I'm looking and learning can I use them [the colours] again? because + yeah (ok) because I speak Telugu too and I chose blue for the nose because I am in New Zealand where they speak English and yeah food again because smell of the food like + the smell of the fresh air also because New Zealand has different kinds of flowers and sometimes you can smell them like to smell really good things yeah

Moh: cool + what about the arms, hands and the shoes?

Lara: so for the shoes I chose both the colours [green and red] because both of them are Indian + you speak them in India and I do dancing so an Indian dance Bharatanatyam so that includes both of the languages cause they also know what that means like + it's part of- yeah cause + cause (that is it) both of the two languages they speak in India and Bharatanatyam is also an Indian dance.

Lara begins explaining the drawing by noting that the dress is blue because it is 'long' (line 36). When I ask her to explain what 'long' means, she indicates that it is 'a big part' (line 38) because she needs it to communicate with people outside the home (lines 39 and 40). Thus, she displays her production of English as linguistic capital that is 'the first thing that comes out of the mouth'. This construction could be because of the influence of her surroundings (e.g. society and school) on her 'habitus'. She then explains that she has coloured the hair red because it is the brain (line 45) where she remembers Telugu (line 46). In line 47, Lara provides an emotionally loaded statement by saying 'it's my mother language, so I always remember it', which shows the linguistic capital that Lara connects to Telugu. Additionally, she displays the cultural capital associated with Telugu through colouring the head red in an Indian headcover-shape (i.e. the Turban). The supplementary clue, i.e. the Turban, from the drawing makes clear that Lara indexes her Indian cultural identity through the cultural capital she displays. For Spanish, she explains that she wants to colour the ears pink (line 48) because she listens to Spanish (line 49). However, she makes a written comment only outside the character to show the Spanish language skill she is practising at school (line 50). Then she explains that the eyes are green because she is 'looking and learning' (line 51). The act of looking at or for Hindi is open to different interpretations. However, as she notes later in lines 64 through 69, this could 
be interpreted as perceiving or exploring other aspects of the Indian heritage through her membership of the wider Indian community in New Zealand, in which the HS plays a significant role. In line 52, Lara wants to add some details to her drawing giving more room for English, Telugu and Hindi as per their associated functions and activities. She explains that she uses red for the mouth because she speaks Telugu (line 56). She explains the blue nose because she lives in an English-speaking country (line 58). She then refers to the sense of smell as a way to perceive New Zealand cultural and natural attributes including food, fresh air and flowers (lines 59 through 61). In so doing, she takes an epistemic stance of knowing and experiencing the New Zealand lifestyle, which is also supplemented by her English linguistic capital and the blue dress, to display her familiarity with and fit in New Zealand society which in turn indexes her New Zealand cultural identity. Furthermore, Lara colours her shoes green and red because 'both of them are Indian' (line 64). Lara offers an analogy of Hindi and Telugu as Indian languages and the 'Bharatanatyam' dance that she dances (line 65). In the subsequent lines (66 through 69), she elaborates on the analogy by noting that Hindi and Telugu are spoken in India and 'Bharatanatyam' is also an Indian dance. Thus, Lara foregrounds the symbolic capital she associates with her Indian languages, which in turn indexes an Indian cultural identity through the pairing of the two Indian languages with the Indian dance.

Lara's linguistic reflection drawing provided subtle details that might not be captured by a verbal description without the additional resource of the drawing activity. The way Lara drew the hair and shoes illuminated the understanding of how she used her languages as a resource to index her Indian cultural and national identities. Lara's description of her drawing revealed her associations of English and Telugu with her linguistic capital, and she incorporated Hindi and Spanish in the linguistic capital to construct her linguistic identity as multilingual. Simultaneously, she associated Telugu and English with her cultural capital and her ability to construct her Indian and New Zealand cultural identities respectively, which in turn indexed her cultural identity as multiple.

\subsection{Conclusion}

This chapter focussed on the HL learners' representation and negotiation of their identities, as well as the language ideologies they displayed towards their languages. This had the purpose of delving into the influence of the HS CofPs membership on their multilingual identity development. In Chapter Four, a glimpse of the CofPs shared repertoire was captured. It showed how the practices within the HS and the dominant language ideologies emphasised the 
Indian identity as an overarching one, i.e. the presentation of Indianness, rather than other identities such as regional, national or religious ones.

In this chapter, the learners used their languages as indices to display forms of capital (Bourdieu, 1986), which in turn supported their identity negotiations. First, the HL learners positioned themselves as Hindi learners with multilingual identities. Yet, there were some students, e.g. Lucy, who did not include Hindi in their repertoire. Second, they typically used the linguistic, social and cultural capitals associated with English to index New Zealand national, and sometimes cultural identities. Third, the learners' heritage languages were mainly associated with linguistic and cultural capitals and indexically tied to the Indian cultural identity. By contrast, Hindi was associated with social and cultural capital along with nationalism (India's national flag) to index the Indian national identity. Fourth, the Indian national identity was accompanied by the Hindi-dominant language ideologies that were visible in the HS practices. Fifth, the HLLs used one kind of identity to index another. For example, Havana constructed her multilingual and multicultural identities and used them as a resource to negotiate her Kiwi identity. Conversely, Lara included Hindi in her multilingual linguistic identities to index her Indianness. Most importantly, the HL learners constructed their multilinguistic identities in ways that made clear that they conceptualised their national and cultural identities as multiple, challenging the notion that national identity is fixed.

The linguistic reflection drawings served as a helpful task for uncovering the language perspectives of young participants and the indexical ties between language(s) and their conceptualisations of 'self'. The participants were excited during the drawing task and provided informative responses. The linguistic reflection drawings also helped the participants to reflect on the emotional and functional aspects of their languages.

In each of the previous two chapters, the data was collected at the HS and so the dominance of these ideologies might be expected. In the next chapter, I move to an additional set of recordings collected in the home where I aimed to access everyday practices of the community members. 


\section{Chapter Six: Language practices in the home}

\subsection{Introduction}

Chapter Four focussed on practices in the Hindi School and how these practices influenced the HLLs' identity construction. Chapter Five then explored the HLLs' perceptions of language and self. This chapter focusses on the language practices within the family. The language practices of three families, reflecting a range of commitment to HLs in the data, are described and analysed. I begin this chapter by discussing the value of language practices in sociolinguistic research. Then, language practices within the Morumaha family are described in Section 6.2, illustrating the negotiation of identities in multiple memberships within a transnational adoptive family. Sections 6.3 and 6.4 discuss language practices in Kevin's and Kayaji's families, capturing how language and identity are negotiated in families with complex linguistic and cultural repertoires.

Language practices play a crucial role in defining identity (Hatoss, 2013; Kumar, 2018; Revis, 2015). Research has shown that identity construction is constituted discursively (Bucholtz \& Hall, 2005; De Cillia, Reisigl, \& Wodak, 1999). Cummins (2000) argues that identity is enacted in interaction through 'identity statements' which often include language attitudes and building connections with other speakers and group members. In the same vein, Le Page and TabouretKeller (1985) argue that speakers enact identities through 'acts of identity' rather than describing them, a point often found in the language maintenance and identity literature (e.g. Morales, 2019). Furthermore, identity statements and acts reveal the speaker's agency in ‘identity negotiation’ in interaction (Albirini, 2016; Eckert, 2000; Rampton, 1995)

Language practices also reflect language beliefs and ideologies whether societal or individual (Canagarajah, 2008; Spolsky, 2004). For example, De Cillia et al. (1999, p. 153), drawing upon Bourdieu's $(1986,1991)$ notion of habitus, argues that national identity is
a complex of common ideas, concepts or perception schemes, (a) of related emotional attitudes intersubjectively shared within a specific group of persons; (b) as well as of similar behavioural dispositions; (c) all of which are internalized through 'national' socialization.

That is, national identities can be understood within discourse, through language and other semiotic practices that construct the social actor's belonging to their imagined national community. Thus, exploring language practices help situate the meaning of the participants' sense of belonging to their communities in contextualised settings. 
Bailey (2007, p. 341) views language as "our primary semiotic tool for representing and negotiating social reality, including social identity categories". This view does not necessarily suggest that speaking the HL is the only means to display HL identity, whether ethnic, national or cultural (Edwards, 2010; Ngaha, 2005, 2011). Language practices not only refer to the language choice in Gee's (1996) 'small-d' discourse, but also 'Big-D' Discourse and related meta discussions (See also Guardado, 2014). In other words, language practices include language use within the family as well as the way the speakers talk about language. In this way, this chapter builds on the previous chapter by exploring how the language is used in context and discussed.

As noted earlier, research in the fields of language maintenance and heritage language has traditionally relied on surveys and interviews (e.g. Kuiper, 2005; Mirvahedi, 2014; Oriyama, 2010; Shameem, 1995; Umali \& Bell, 2017). While these methods of data collection have been useful to gather data from large samples (Holmes, 1997), recent practices highlight the importance of ethnography in capturing actual practices rather than reported behaviour (Goodz, 1994; King \& Logan-Terry, 2008) aiming at a more nuanced understanding of the complexity (Rampton, 2007). Since the role of socialisation among family members is influential for HLLs' identity construction (Li, 2006; Lo-Philip, 2010; Seals, 2013, 2017b), the inclusion of naturally occurring home interaction, where HLLs socialise and are socialised with, has the potential to illuminate our understanding of the ongoing identity construction and negotiation in the participants' most comfortable and central context, i.e. the home (Revis, 2015; Seals, 2019; Tyrrell, Guijarro-Fuentes, \& Blandon, 2014). Furthermore, Lanza (1992, p. 201) emphasises the value of home data, advising that research needs to "focus on the micro-level of interaction [...] through a discourse perspective on language contact in parent-child (caregiver-child) interaction". While the data collected from ethnographic work throughout my research offered a holistic view of the community practices and their influence on HLLs, the premise for including home recording lies in my recognition of the diversity of families in the community. By looking at a range of families, I hope to capture a range of different family practices (cf. family language policy, see for example Macalister \& Mirvahedi, 2017). In order to investigate the actual practices of these families, naturally occurring interaction at home was recorded by the parents themselves.

As noted in Chapter Three (section 3.3), the data was collected within three families, representing the different kind of families within my data. I here introduce these families on two bases, namely, the family's demographics (specifically their links with India) and their 
commitment to the HL. The first family represented an adoptive family, but highly committed to HL. The family comprised two parents originally from Europe (Mark and Sara), who came to New Zealand some ten years ago. They adopted Muromaha seven months before the data collection. Muromaha was seven when she came to New Zealand to live with her new family. The second family was a mixed and multiculturally committed family, which was comprised of a Fiji-Indian father, Kevin, a Chinese mother, Grace and three children (Havan, Austin, and Max). The third family was a mixed Indian family, with multiple Indian languages. This family included an English-speaking father (of Indian descent), Peter, a Fiji-Indian mother, Kaya ji and two daughters (Ann and Mylu) and they had a strong connection with the extended family.

\subsection{Muromaha's family}

In this section, I describe and analyse the language practices in Muromaha's family. I discuss how metadiscourse as a language practice was used in constructing and negotiating national and cultural identities, and a general sense of 'Indianness'. I also outline how the family used linguistic meta-discussions to connect Muromaha with her heritage language and identity.

The home interaction data in Muromaha's family included 351 minutes (five hours and 51 minutes) of recordings. As is the case with social sciences research, especially in sociolinguistic research, recording of conversations in this family mostly took place at the dinner table because it was an opportunity for family members to sit together and socialise (Fogle \& King, 2013; Gerhardt, 2013; Kheirkhah \& Cekaite, 2015; Raspayeva, 2018; Revis, 2015; Sierra, 2018). Mealtime was also considered a site for extended discourse (Snow \& Beals, 2006, p. 54). In addition to mealtime, Muromaha's family also recorded their interactions during playtime which provided a context of socialisation among family members (Gordon, 2009; Revis, 2015).

Research has shown that transnational adoptive families often make more HL-related decisions than bi- or monolingual families do when the linguistic and cultural backgrounds of their adoptees are different from their own (Fogle, 2013). In this family, Muromaha spoke Hindi as her first and only language when she arrived in New Zealand. Being enrolled in primary school and being socialised by her parents who spoke English, her parents made decisions such as enrolling Muromaha in a language maintenance school (cf. Shin, 2011). Other decisions included employing a language tutor (Barkhuizen, 2015) who could socialise with Muromaha in a more naturalistic way in Hindi, helping Muromaha in the transition to her new cultural environment Fogle (2013). At the same time, these decisions paralleled the parents' wishes for 
English language development (Shin, 2013). These decisions were directed towards the development of the child's "Indian" identity as well as "reinterpreting [the parents'] own racial and cultural identities" (Shin, 2013, p. 261). During the time of data collection, Sara and I had a conversation about Muromaha's background in which Sara positioned her as a Hindi speaker who was learning English. However, Muromaha typically positioned herself differently, exhibiting a complicated, sophisticated and agentive identity construction in the data. As will be shown in what follows, Muromaha demonstrated an ability to (dis)align with the way she was positioned by the parents at times.

Metadiscourse has an important role in interaction as it guides the interlocutor's perception of the discourse (Hyland, 2017, 2019; Hyland \& Tse, 2004). Findings from heritage language research have shown that metadiscourse constitutes and enacts the caregivers' sense of responsibility to pass the language to the children. In his discussion of discourse of identity, Guardado (2018a) provides examples of family metadiscourse such as pride and access discourse. This metadiscourse can include metalinguistic comments which the participants use as an interactional mechanism to negotiate and resist family language policies (Fogle \& King, 2013; Guardado, 2018b; Lanza, 2004; Revis, 2017).

While emerging FLP research highlights the relationship between metalinguistic discussions and the negotiation of language choice within the family (Fogle \& King, 2013; SmithChristmas, 2016), the home data from Muromaha's family demonstrated that metalinguistic comments were used as an 'act of identity' in themselves (Le Page \& Tabouret-Keller, 1985), which more or less corresponded to Revis' $(2017$, p. 7) findings that metalinguistic comments in the Columbian and Ethiopian families were targeted at ethnic identification purposes. Additionally, research on socialisation has noted that metalinguistic talk and comments reflect high levels of linguistic awareness regarding language forms, appropriate language use and 'correct' usage (Blum-Kulka, Huck-Taglicht, \& Avni, 2004, p. 317; Blum-Kulka \& Snow, 2004). In addition to linguistic awareness, Schwartz and Gorbatt (2016, p. 670) note that metalinguistic discourse involves jokes, and "provides a foundation for creating of novel friendships, personal empowerment, and pleasure." They further conclude that metalinguistic discourse reflects the children's "agency role in shaping the linguistic environment and language policy" (Schwartz \& Gorbatt, 2016, p. 685) and, potentially allows for identity work. The data showed that meta-discussions on Muromaha's Indian identity and Hindi were regular and salient, and although Muromaha's parents encouraged her Indian identity through meta- 
discussions, there were times when Muromaha seemed to respond negatively and disalign herself with being positioned as an Indian.

\subsubsection{Meta-discussion around national identity}

Meta-discussions around Muromaha's national identity signalled that her Indian identity occupied a significant part of her parents' discourse in the data. The home recordings showed that Muromaha's parents supported her Indian identity and were highly committed to keeping her connected with her Indian heritage. They seemed not only to acknowledge her Indian identity, but also to view it as recognized by the others. They also connected Muromaha with her Indian roots whenever they felt possible. They connected her with India through Indian artefacts, music, food, and Hindi.

Meta-discussions, as the prefix 'meta' suggests, reflect the interactants' awareness of the topic being discussed. In this case, the family was highly aware of Muromaha's Indian identity since she was Indian-born and had recently come to New Zealand. In addition, meta-discussions could be seen as a site for deliberate identity negotiation where individuals (dis)align, accept or reject certain identities. While meta-discussions functioned in this way across the data, this constant positioning seemed to be problematic for Muromaha perhaps because it created a sense of threat.

\subsubsection{Positioning leading to othering}

Positioning draws on how individuals identify themselves and others discursively in conversational encounters (Bronwyn Davies \& Harré, 1990; Langenhove \& Harré, 1999). According to positioning theory, social actors use "language displays of oneself" (Howie \& Peters, 1996, p. 53) to locate themselves and others in discursive activities and these positionings reflect their ideologies and emotions among other embedded cultural meanings (Hatoss, 2012, p. 50). Building on a general finding that individuals are constantly positioned and repositioned in interaction, research has shown the impact of positioning on identity. Seals (2013) studied the influence of positioning HLLs of Russian on their linguistic identity development and negotiation and found that "the ways that students were positioned by their parents and teachers strongly affected their own multilingual identity negotiation" (Seals, 2013, p. 205). Similarly, the studies conducted by Martin-Beltrán (2010) and Umansky (2016) revealed that positioning young language learners (HL or otherwise) affected their language proficiency and membership in the various communities they belonged to. These studies and many others examined the construction of participants' linguistic identities when positioned by those who were viewed as linguistically more expert than the learners themselves. 
Nonetheless, the case seemed to be different when the interactional partners had limited or no linguistic knowledge in the language of the young learner/speaker, as the data about some adoptive families revealed. In Muromaha's case in the present study, there were instances where she was positioned as a speaker of Hindi. Muromaha was positioned by her mother, Sara, as a Hindi speaker ("Hindi is her first language"). During the observation period in the HS, the teacher introduced Muromaha as "a new Indian girl" who "knows Hindi well" but she was temporarily placed in the entrance class because she had joined the school recently (Fieldnotes 27 August, 2017).

While the positioning put Muromaha at the centre of the Hindi speaking community, research showed that individuals could experience a kind of positioning, particularly 'othering', that influenced their national, cultural or ethnic identity construction. In her study of Sudanese former refugees in Australia, Hatoss (2012) concluded that her participants were 'othered' by the mainstream culture despite their disposition to integrate into the Australian society, which in turn, as Udah (2018, p. 397) suggested, had the potential to lead to the community failing to integrate. While these studies have looked at how minorities are positioned as others by the mainstream cultures, othering can be practised within the family, even if unwittingly. Experiences like this appeared to garner various reactions from family members as in the following example. Excerpt 6.1 comes from a discussion in which Muromaha (Muro in the excerpts) recounts what she has done at school. The family had a set daily practice of tellabout-the-day (Fogle \& King, 2013), which was part of the family language policy since it was oriented towards practising English, inter alia. In Excerpt 6.1, when Muromaha tells them about hair clips that her friend Kessey has given her, the family engage in a discussion of Muromaha's Indian identity.

Excerpt 6.1: "Do you think she gave them to you because you're Indian?" (Home Recording)

1 Sara: why did Kessey give you um: the clips?

2 Muro: I dunno + but she told me they're from India

3 Sara: so it's from India, is it?

4 Muro: Ha

5 Sara: how beautiful + ( ) do you like them?

6 Muro: hm [agreeing]

7 Sara: do you think she gave them to you because you're Indian? is that why she?

8 Muro: I don't know

9 Sara: Em

In line 1, Sara encourages Muromaha to wonder why Kessey gave her the hair clips, a question that receives a vague answer with a hint that the clips are made in India (line 2). Seemingly, Sara has taken Muromaha's response in line 2 as a potential reason for receiving the clips. It is 
noteworthy that across the data Muromaha gives 'I dunno' (and its variants) as a response to many questions that her parents (especially Sara) ask about her Indian identity, perhaps when she feels that her Indianness threatens her membership in the family. This interpretation was confirmed in the post-recording feedback with Sara. It would seem that Sara strategically uses the rhetorical question tag in line 3 and the expression of liking (line 5) to implicitly persuade Muromaha to align with what is being said in line 7. Sara allows room for considering Muromaha's Indian identity as the reason for Kessey's action by presenting the idea in the form of a yes/no question rather than a statement. Yet, Muromaha seems not to align herself with what Sara is saying.

While the parents' deliberate foregrounding of Muromaha's Indian identity is ostensibly targeted at their commitment to locate Muromaha within her heritage, Muromaha often has different interpretations. As illustrated in Excerpt 6.1, while Sara positions Muromaha as an Indian and this positioning becomes the theme of the sequence of turns, I would argue that Muromaha interprets Sara's talk as 'othering'.

\subsubsection{Rejection of positioning (being othered)}

When considering positioning we need to recognise the influence of power relations between interlocutors. This means rejection, resistance and negotiations are sometimes impossible or challenging (Blackledge \& Pavlenko, 2001). Hatoss (2012, p. 65) has suggested that positioning in contexts where acts of identity are performed in interethnic and high-risk settings (e.g. everyday life) yields different reactions to contexts where identity work is enacted on safe ground (e.g. sociolinguistic interviews). Like sociolinguistic interviews, the family domain is expected to be a safe context in which participants may perform unrestricted identity work.

In their daily interactions, Muromaha's family regularly had conversations around Muromaha's Indian identity. I argue that these conversations created a sense of discomfort to Muromaha, and at times she refrained from contributing to the conversation. Excerpt 6.2 is an example. The excerpt is part of a conversation at the dinner table when Muromaha turned the CD player on, played Indian music and joined the family for dinner. The main topic of the excerpt is Muromaha's choice of music.

Excerpt 6.2: "why did you put this disc this CD on today?" (Home Recording)

$\begin{array}{lll} & & \text { [Indian music in the background] } \\ 1 & \text { Sara: } & \text { why did you put this disc- this CD on today? } \\ 2 & \text { Muro: } & \text { I dunno } \\ 3 & \text { Sara: } & \text { the food is quite tasty } \\ 4 & \text { Mark: } & \text { it's nice } \\ 5 & \text { Muro: } & \text { I dunno why + I didn't think }\end{array}$


6 Sara: I think you like it because it's Indian music

7 Muro: why do //you?

8 Mark: /I thinkll you like it ++ you've got a nice choice

9 Sara: hm! so what do you think

10 Muro: I dunno + I think we should put it on

11 because we haven't listened to it for a long time

12 Sara: no you like to listen to this sweetie + don't you?

13 Muro: Hmm

When Muromaha returns to her seat, Sara initiates the conversation by asking why Muromaha wants to play Indian music specially (line 1). Sara seems to indirectly connect to Muromaha's Indian identity by posing the question 'why did you put this disc-this $C D$ on today?' rather than giving a direct statement. However, Muromaha responds negatively in lines 2 and 5 by saying 'I dunno'. I dunno is a regular response from Muromaha and seems to function as a way to avoid further questions (Baumgarten \& House, 2010) and resist the parent's request (Guardado, 2013). In line 6, Sara becomes more explicit, giving her own evaluation (Baumgarten \& House, 2010) telling Muromaha that she thinks the reason for playing the music is that because it is Indian music. In response to this statement, Muromaha's question of why Sara has such an opinion (line 7) sounds to be another form of resistance to the continuation of positioning her as an 'other'. Mark then contributes to the interaction seemingly with the intention of alleviating Sara's insistence and the daughter's resistance. He suggests that Muromaha likes the music and compliments her on her choice (line 8). However, Sara once again insists on knowing what Muromaha thinks by repeating the question in line 9 . Again, Muromaha responds with 'I dunno' (line 10) and elaborates that they have not listened to it for a long time (line 11). Sara responds to Muromaha's idea with a direct rejection by using no at the start of line 12 as well as taking control of the conversation.

The response, 'I dunno', is constantly used by Muromaha, arguably functioning as a stancetaking sign that indexes avoidance (See also Baumgarten \& House, 2010) which, I suggest, is because Muromaha interprets herself as being 'othered', however unintentionally. The excerpt shows that despite the inequity in power relations between her and Sara, Muromaha shows resistance and reluctance towards the notion of overemphasizing her Indian identity in certain contexts (turns $2,5,7,10$ ). She is actively expressing her agentive role in the language practices (Revis, 2017). In fact, Muromaha's reluctance towards what she feels as 'othering' was reported in the post-recording feedback with the family and she has recently stopped going to the HS. The negotiation of Muromaha's stance in relation to being positioned as Indian (and thus othered in her views) takes the forms of contestation and avoidance. Like her responses in Excerpt 6.1, she uses vague responses and questions to avoid further engagement in the 
conversation in Excerpt 6.2. Although the process of othering is often based on phenotypical characteristics such as colour (Hatoss, 2012; Udah, 2018) and negative stereotypes (MacNaughton, Davis, \& Smith, 2009), Muromaha's rejection of being positioned as Indian might derive from her understanding of the repeated attempts to connect her with her Indian heritage as an interruption to her engagement in the family. At other times, however, it was evident in the data that she does not resist and reject her Indian identity. The following section demonstrates how Muromaha shows agentive identity construction in relation to the communities she belongs to.

\subsubsection{Complex and contradicting membership}

The experiences that Muromaha had in India and New Zealand were expected to offer her a multiple sense of belonging. Being born and raised in India might have established her Indian identity. Similarly, her life in New Zealand where she lived with her adoptive parents and went to school could be seen as a source for creating a common identity as a member of the family and an ingroup member in the home and school respectively.

In Excerpt 6.3, the family engage in a conversation that shows Muromaha's membership in her various and overlapping communities, i.e. the family, Indian and school communities. In the excerpt, Muromaha recounts her school visit to Te Papa, the national museum of New Zealand, to her parents. During the school visit to the museum, Muromaha painted a bridge using painting software. She is explaining to her parents how she painted the bridge in the excerpt.

Excerpt 6.3: "I was going to say India but that's what she said" (Home Recording)

\begin{tabular}{lll}
\hline 1 & Muro: & {$[\ldots]$ then after it says put your name then next where are you from } \\
2 & & then after next um why do you like it \\
3 & Sara: & did you put where you're from? \\
4 & Muro: & hm Wellington \\
5 & Sara: & good yeah \\
6 & Muro: & because that's what Shishma said \\
7 & & I was going to say India but that's what she said \\
8 & Sara: & because that's where we the three live eh? \\
9 & Muro: & yeah \\
\hline
\end{tabular}

As shown in Excerpt 6.3, Sara explicitly displays her interest in knowing how Muromaha identified herself in response to the software questions (line 3). In line 4, Muromaha reports that she identifies herself as a Wellingtonian and then elaborates that she has done that because her friend said to (line 6). Although the question of where someone is from could mean 'where do you live?' or 'where are you (originally) from?', Muromaha opts for the latter. Line 7 suggests that if Muromaha were not to 'accommodate' (Giles, 1973) to her peer, she would identify herself as an Indian. Muromaha highlights the influence of peers in her explanation, 
and although she identifies herself as an Indian using an 'identity statement' (Cummins, 2000), she shows solidarity with her peer, Shishma, by selecting the same answer, which in turn serves as an act of asserting a common identity (Le Page \& Tabouret-Keller, 1985). Sara opts for the former by praising Muromaha's response (line 5). The act of praising is presumably targeted at supporting Muromaha's identity as a local (rather than migrant). She also supports Muromaha's membership in the family by explaining that Wellington is 'where we the three live' in line 8. In line 9, Muromaha agrees with Sara's statement. This example clearly reflects Muromaha's understanding of her complex membership in several communities (i.e. as a family member, Indian and Wellingtonian), but it also shows the developing identity that is emerging in Muromaha's discourse.

Overall, these examples showed the dynamic and multiple nature of identity construction and negotiation (Darvin \& Norton, 2015; Hua, 2017; Skutnabb-Kangas, 2000) as a by-product of Muromaha's engagement with how she was positioned in relation to her belonging in her communities. Excerpt 6.3 suggested that Muromaha was aware of and acknowledged her Indian identity, but also recognised her 'local' identity (i.e. as a Wellingtonian) with which she identified alongside her friends at school. She also valued her membership in the family. Returning to Excerpt 6.1 and Excerpt 6.2, it seems that one of the contexts in which Muromaha disaligned with being positioned as an Indian was when she felt her Indian identity interpolated her status as a member of the family. Disaligning with being positioned in this way (i.e. being othered as she might think) did not necessarily lead her to rejecting her Indian identity. Rather, her rejection was reflected in her avoidance of engaging with her parents in the interaction. When her status as a family member was not at stake, she displayed her Indian identity.

The complex and sometimes contradicting membership in the different communities suggests that relevant identities are discursively available. The following section outlines how the family engaged with the cultural values related to these identities.

\subsubsection{Meta-discussion on cultural signals}

Cultural signals, e.g. attitudes, preferences, knowledge, behaviours, goods and credentials (Lamont \& Lareau, 1988), and values are foregrounded in heritage language research (Ayeomoni, 2011; Brown \& Carpenter, 2018; Dweik et al., 2019; Hua, 2014; Revis, 2015; Shameem, 1995). Guardado (2008) noted that parents socialized their children into their cultural values and practices. In their study of the language practices within Spanish-speaking families in Britain, Tyrrell et al. (2014) found that parents discussed language and cultural values with their children and reminisce about their own childhood cultural experiences. When 
it comes to adoptive families, research suggested that the adoptive parents' efforts to keep the adoptee's heritage culture mitigated some of the challenges of living in a multicultural family (Shin, 2014; Volkman, 2005). In Muromaha's case, although her parents did not share the same heritage, they appeared in the recording to encourage her to talk about Indian cultural aspects, especially those shared with New Zealand. For example, Sara once engaged her in a conversation on sports and then she asked Muromaha about playing cricket in India. Excerpt 6.4 is an example of the parents incorporating Muromaha's life experience in India regarding food. The family started the conversation over the soup that Mark prepared and then shifted to talking about the noodles that Muromaha used to have in India in comparison with the noodles she was having at dinner.

Excerpt 6.4: "it's unlike the noodles we had in India" (Home Recording)

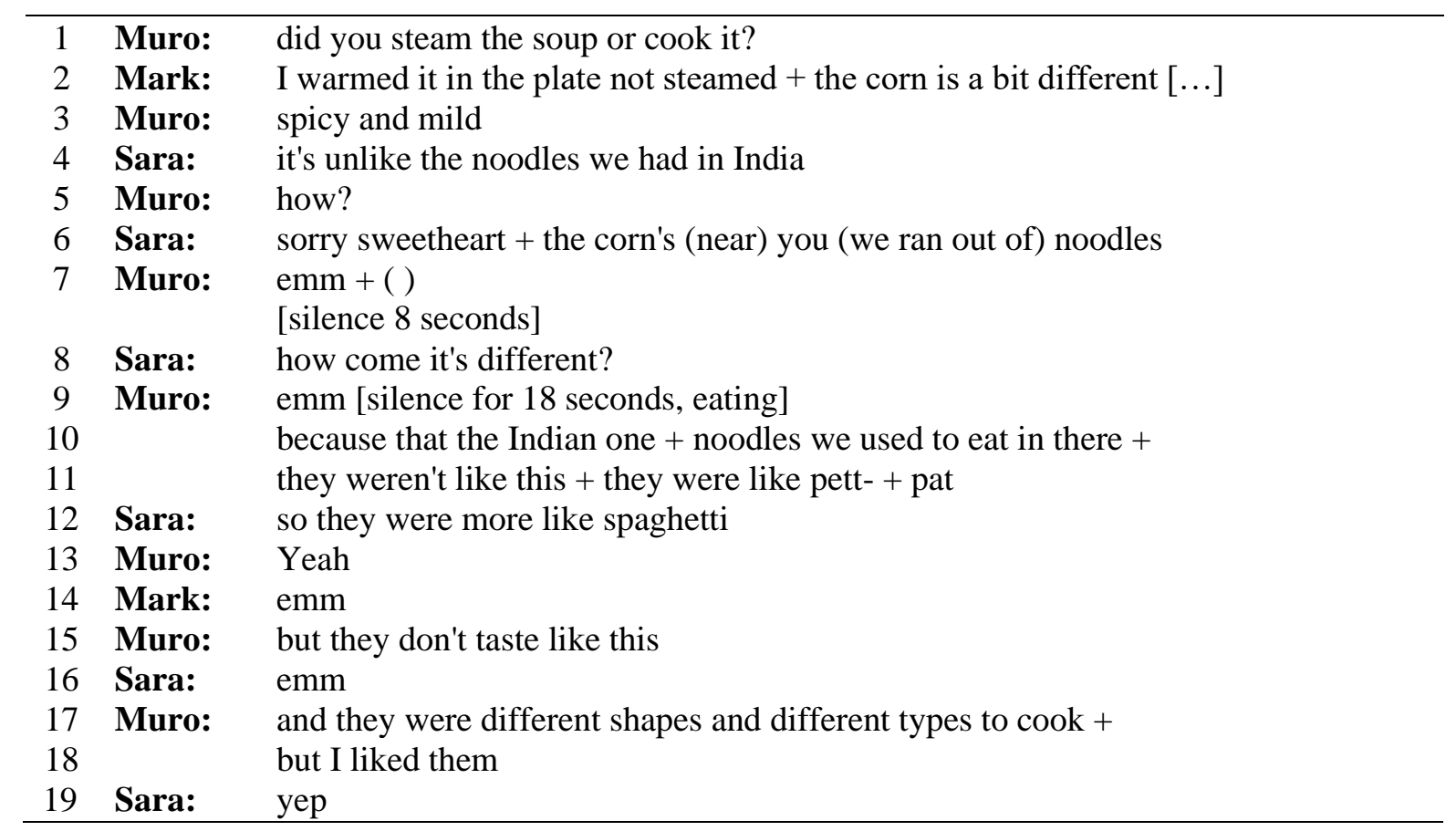

Sara regularly initiates the connection to India. In line 4, she takes the chance to recall the family's experience of having noodles when they visited in India. While Muromaha displays interest in knowing how this meal is different (line 5), Sara interrupts the flow of the conversation, perhaps unintentionally. Then in line 8, Sara connects the food with Muromaha's own experience in India by asking her to explain the differences between the two meals. In so doing, Sara is positioning Muromaha as an expert on this topic. From line 9, Muromaha actively contributes to the interaction showing agreement with Sara, expressed by backchannelling in lines 7, 9 and 13, and adding information in lines 10, 11, 15, 17 and 18. In listening to the interaction it seems that Muromaha feels comfortable being positioned as an 
expert in the food she experienced in India. Interestingly, giving Muromaha the chance to be the knowledgeable partner resonates with linguistic practices of caregivers in other communities (e.g. Guo, 2014; Tyrrell et al., 2014) in the sense that they switch interactional power relations with the children. In this regard, Guo (2014) reported in her study of Mandarinspeaking families in the UK that the children in those families taught their parents mainstream cultural concepts, including food.

In addition to their commitment to keeping Muromaha's heritage foregrounded, Muromaha's parents seemed to have a 'here and now' orientation to her life in New Zealand. The recordings included examples of discussing cultural signals related to her life in the communities she belonged to in New Zealand. At one point, Muromaha commented that my name (Mohammed) was strange. Sara explained the religious origin of the name and gave an example of other naming practices from the Catholic culture. On other occasions, the family engaged in a discussion of their own travel experiences, dietary habits (e.g. being vegan) and making friends. Excerpt 6.5 exemplifies the discussion of socialising with other children at school. In the excerpt, Muromaha recounts her first day of the school year to her parents. She points out that a new student has joined the class. By showing a desire to know how she engages with the newcomers at school, the family get into a discussion on the norms of socialisation.

Excerpt 6.5: "but this is a very good opportunity for you to show that you're friendly and nice" (Home Recording)

\begin{tabular}{cll}
\hline 1 & Sara: & did you go and talk to her? \\
2 & & [Muromaha remains silent] \\
3 & Sara: & well she's a new girl like you //were last term \\
4 & Muro: & /but not nowll \\
5 & Sara: & no but that's a good opportunity for you + yes? \\
6 & Muro: & but she's been here before + in this class \\
7 & & because she probably she was here last time \\
8 & Sara: & huh! but did the others recognise + did the others know her? \\
9 & Muro: & probably + Torra and Emilia + probably they did \\
10 & Sara: & well- because they were talking to her \\
11 & Muro: & yes \\
12 & Sara: & it might be that Torra and Emilia asked because they were on their first day \\
13 & & remember when Koko + when you first started + Koko was asked to be your little \\
14 & & friend //and \\
15 & Muro: & /butll because Kef [the school teacher] asked her if you remember \\
16 & & [...] \\
17 & Sara: & you might find in the next few days that she doesn't have anybody to chat to \\
18 & Muro: & I'll see tomorrow \\
19 & Sara: & you'll see tomorrow? \\
20 & & but this is a very good opportunity for you to show that you're friendly and nice and \\
21 & & yeah? and that you're willing to play \\
22 & Muro: & I AM \\
23 & Sara: & YES? THAT you are + YOU ARE? \\
\hline
\end{tabular}


The positioning appears to start when Sara interrupts Muromaha's flow of speech about the new child at school. Sara's curiosity to know whether Muromaha has spoken to the newcomer is expressed by a question in line 1. Muromaha's silence in line 2 suggests that she strategically uses silence to manage the conversation (Jaworski, 1993) by refraining from answering the question as a way of rejecting it (Jaworski, 1998). Sara models the shared practice in the school/community, drawing upon Muromaha's experience as a former newcomer by reminding Muromaha that she was new to the school (line 3). While Sara wishes to connect Muromaha with features of the (New Zealand) identity within the school context, it seems that Muromaha attends to Sara's guidance as positioning her as an outsider (and thus as an 'other'). Muromaha displays her status as an insider in the school community by interrupting Sara saying 'but not now' in line 4. Once again, this suggests that Muromaha resists further interaction when her membership in the school community seems to be threatened.

The interaction between Muromaha and Sara shows rejection of what the other is saying. This rejection is marked through the direct 'no' (line 5) and the use of the conjunction 'but' at the beginning of the turns (line 4, 6, 8, 13, 18) as well as Sara's repetition of Muromaha's words in the form of rhetorical questions (lines 17 and 21). In line 5, Sara is directly rejecting the way Muromaha perceives her words. She controls the conversation, shifting to highlighting the advantage of talking to the new child at school. Again, Muromaha resists the idea of having to talk to the new child by providing a justification for her viewpoint (line 6 and 7). In line 13, Sara once again refers to the previous year when Muromaha first joined the school and one of the classmates, Koko, talked to her. In so doing, Sara foregrounds socialising with new children as a norm and shared practice. The model used by Sara arguably suggests that she is trying to connect Muromaha to the New Zealand identity in which friendliness is seen as a potential marker (Holmes, Marra, \& Schnurr, 2008; See also Wohlfart, 2017), especially in Muromaha's school context. Sara explicitly explains the view of showing friendliness in lines 20 and 21. Nonetheless, Muromaha consistently demonstrates resistance to what is being said. In response to Sara's argument, Muromaha stresses that she is friendly and nice by saying ' $I A M$ ' in a high pitch, which leads Sara to reject Muromaha's claim in the form of a rhetorical question in line 23.

As noted earlier, Muromaha's membership in multiple communities allowed the family to engage with the cultures of these communities. While the example in Excerpt 6.4 illustrated their engagement with the culture Muromaha experienced in India, Excerpt 6.5 exemplified 
the discussion of socialising with other children at school as a cultural value in at least in Muromaha's primary school if not in wider New Zealand society. Excerpt 6.5 also showed how Muromaha rejected what she seemed to consider positioning as 'other' concerning her social behaviour in the school community and negotiated this positioning. Like the example presented in Excerpt 6.3, the rejection was targeted at what she saw as a threat to her membership in the school community. The interaction also highlighted discursive "negative identity practices" (Bucholtz, 1999) when Muromaha felt that she was being 'othered'. I argue that Muromaha did not reject being positioned as Indian, nor did she reject her Indian identity. Rather she resists and rejects being 'othered'. Excerpts 6.1 through 6.5 showed that Muromaha rejected being positioned as Indian only when she felt that she was being seen as 'different' from her surroundings (e.g. family, school), and although her parents were supporting her Indian identity, it might have not occurred to them that they were seen as othering her. In the following section, I provide evidence to support this argument from the recordings where the family engaged in linguistic meta-discussions regarding Hindi.

\subsubsection{Linguistic meta-discussion (positioning child as an expert)}

Linguistic meta-discussions were salient in the home interaction data, with a focus on English and Hindi. There was also one instance when Sara discussed her Te Reo speaking ability, asking Muromaha when do you reckon you'll be speaking Māori quite well?, suggesting the value placed on Te Reo as a marker of 'New Zealanderness'. Meta-discussion of English occurred as comments on Muromaha's language production and explanation of vocabulary. The family appeared to recognise Muromaha's linguistic identity and engaged in linguistic meta-discussions about Hindi. Excerpt 6.6 is an example. Muromaha and her father are playing a game in which one player asks a question and if other player answers correctly, they get one point, otherwise, they get a blank. In this excerpt, Muromaha uses her knowledge in Hindi to win the game, but the game turns into a discussion of pronunciation.

Excerpt 6.6: "he says gaajer, it should be gaajaR" (Home Recording)

\begin{tabular}{cll}
\hline 1 & Muro: & what colour did I ask you in Hindi before? \\
2 & Mark: & hmm \\
3 & Muro: & but you learnt them \\
4 & Mark: & gajerr [carrot-like orange] \\
5 & Muro: & gaajerrrr [laughter, repeating Mark's pronunciation style] \\
6 & Mark: & I am not sure how they say it + in Hindi \\
7 & Muro: & [laughs] \\
8 & Mark: & they roll their r quite (backwards) \\
9 & Muro & gaajaR* + can you say it? \\
10 & Mark: & kaajar \\
11 & Muro: & R + gaajaR
\end{tabular}


12 Mark: yeah

13 Muro: he says gaajer + it should be gaajaR + they might understand the gaajer

14 Mark: it might be enough to get it right but not + not perfect + not perfect Hindi

* alveolar trill

As shown in Excerpt 6.6, Muromaha takes advantage of her knowledge in Hindi to spot the chance of asking a difficult question. She asks Mark to say the colour he has learnt in Hindi (line 1). When he fails to remember it, she takes an epistemic stance by encouraging him to remember it because he has already learnt it (line 3). Mark seems to support the stance Muromaha takes by answering her question in line 4. The repetition of Mark's pronunciation of the word along with the laughter (lines 5 and 7) could arguably be interpreted as performing a Hindi speaker identity as well as indexing Mark's poor linguistic performance in Hindi. In turn, Mark's discursive face-saving behaviour (lines 6 and 8) suggests he not only mitigates his stance, but also aligns himself with Muromaha's Hindi speaker identity. In lines 9 and 11, Muromaha continues performing her linguistic identity as a legitimate Hindi speaker by providing corrective feedback (Tyrrell et al., 2014), modelling the correct pronunciation for Mark who positively responds to the modelling in lines 10 and 12. When Muromaha addresses Sara evaluating Mark's performance in Hindi, she extends this identity to comment on the proper pronunciation of the word and the possibility that Hindi speakers might understand the form he is producing (line 13). Muromaha takes this epistemic stance and displays her knowledge in Hindi to index her Hindi-speaking identity, and thus indexes her Indian identity, as shown in the next excerpt. This behaviour is comparable to the rejection she displayed in the previous examples. Another example is illustrated in Excerpt 6.7. Muromaha and Mark continue playing the game they started in Excerpt 6.6.

Excerpt 6.7: "Indian people are like me but other people are like daddy" (Home Recording)

\begin{tabular}{lll}
\hline 15 & Muro: & and window in Hindi? \\
16 & Mark: & aha! [surprised] \\
17 & Mark: & window? \\
18 & Muro: & em! in Hindi \\
19 & Mark: & [clears his throat] \\
20 & Muro: & I know you're working well + oh! \\
21 & Mark: & can I remember? then I am saying it right \\
22 & Muro: & you won't remember because we haven't done Hindi for a long time \\
23 & Mark: & and I forget \\
24 & Muro: & when I do that + that will be normal Hindi then we don't speak anything else \\
25 & Mark: & ok \\
26 & Muro: & what's the word for that in Hindi? \\
27 & Mark: & how does it start? \\
28 & Muro: & khi \\
29 & Mark: & ok + is it ki- ? \\
30 & Muro: & I know you're going to say \\
31 & Mark: & kirkirr
\end{tabular}


32 Muro: no you're going to say kerky

33 Mark: kirky that's it

34 Muro: no it's wrong

35 Mark: oh that's wrong!

36 Muro: khidhki: [window]

37 Mark: kidki:

38 Muro: no + that's kidki: + khidhki: khidhki:

39 Sara: khidki:

40 Muro: no + it's not + you're like daddy

41 Indian people are like me but other people are like daddy

42 Mark: [cough] let's ask my Indian friend at work +

43

44 Muro:

45 Mark: /he's just a man at workll at work who is a- speaks Hindi

46 Muro: uh! [surprised]

47 Mark: don't you think he's got some children?

$48 \quad$ oh yes + I can ask him my- and get my Hindi right

49 Muro: he might he might not understand what's khidhki: + khidhki:

50 Mark: he might not understand but he might understand

In Excerpt 6.7, Muromaha continues the epistemic stance she has taken earlier in the game (in Excerpt 6.6) by recognising the reason why Mark has forgotten the Hindi word (line 22). Likewise, she seems to use this kind of stance to draw a separation line between her linguistic identity as a speaker of 'normal Hindi' (line 24) who speaks like 'Indian people' (line 41) and her parents who speak like 'other people' (line 41). In doing so, Muromaha might be using a 'positive identity practice' (Bucholtz, 1999) to claim the Indian identity as well as using a negative identity practice to separate herself from her parents in relation to the Hindi linguistic identity. Paradoxically, while she has previously rejected being positioned as 'other', she seems to position her parents linguistically as 'other' in line 41. This is, perhaps, because she feels that language does not threaten their membership in the family. In fact, this finding parallels language maintenance and heritage language research findings where language (proficiency) is not necessarily equated with one's identity or membership in a certain group (Canagarajah, 2013; Dweik et al., 2019; Holmes et al., 1993; Ngaha, 2005).

Notably, the turns in lines 29 through 40 are replete with modelling and corrections of Mark's pronunciation of the word 'khidhki:'. This suggests the bi-directional positioning of both interlocutors. Muromaha positions herself as a Hindi speaker who is able and entitled to demonstrate expert linguistic knowledge (lines 29, 36 and 38), and she is being positioned the same way by Mark (lines 33 and 37) and Sara (line 39). In line 41, Muromaha foregrounds her Indian identity by commenting on shared linguistic knowledge (i.e. pronunciation) with Indian people by portraying language as an identity marker. This corresponds to the finding that minority language speakers use language to show in-group similarities with others (e.g. 
Spolsky, 2012; Starks et al., 2005). Mark aligns with Muromaha's identity by referring to his Indian co-worker, who is a potential expert in Hindi and can assess whether the parents' pronunciation is acceptable (lines 42 and 43). Another possibility is that he is enlisting his Indian co-worker's help (See also Shin, 2014). Despite that, Muromaha performs a strong act of identity by claiming exclusive ownership of the language in line 49 by questioning Mark's co-worker's knowledge in Hindi. In doing so, Muromaha seems to push Mark's co-worker outside the conversation. She also seems to have a mindset that assumes that her Hindi is the standard variety. Interestingly, the Hindi language transcribers who listened to Muromaha reported that her Hindi was quite 'different' from what they normally used and heard, suggesting that she could be a speaker of a regional dialect of Hindi, or at least had child competence only.

The parents positioned her as an expert in Hindi and Indian food (see Excerpt 6.4), which in turn underpinned her Indian identity. This resonates with the finding that allowing the children to be experts in the HL connects to their linguistic and ethnic identities (Desmond, 2017; Kheirkhah \& Cekaite, 2015; Seals, 2013). Regarding the corrective feedback that Muromaha engaged in with her parents, Tyrrell et al. (2014) pointed out that this practice reversed the power roles in the family and gave the child the status of being expert, corrector and teacher. Unlike the Ethiopian parents who avoided teaching Amharic and English literacy skills to their children at the same time in a similar analysis of home recordings by Revis (2015), Muromaha's parents attempted to teach her Hindi and English literacy skills together. In addition to enrolling her in the HS, they tried to make the home a Hindi space. This does not mean that they ignored the family language ecology (Fogle, 2013); instead, they hired a Hindi private tutor and established a practice whereby Muromaha said grace. It was quite habitual for Muromaha's family to turn the recorder on at the table as they mostly socialised while having dinner. Having Muromaha say grace either in English or Hindi was a common practice before dinner. It was Muromaha who made the language choice when saying grace. However, the recorded interaction in Excerpt 6.8 is a result of requesting her to say grace in Hindi while the private Hindi tutor, Ateesha, was present.

Excerpt 6.8: Muromaha says grace (Home Recording)

$\begin{array}{lll}\text { 1 } & \text { Muro: } & \text { [saying grace in Hindi unclearly] } \\ 2 & \text { Ateesha: } & \text { what did she say? } \\ 3 & \text { Sara: } & \text { not sure not sure } \\ 4 & & \text { what was it? say it again } \\ 5 & \text { Muro: } & \text { emm [grouching] } \\ 6 & \text { Ateesha: } & \text { I didn't- I didn't hear anything } \\ 7 & & \text { can you tell me again? }\end{array}$




$\begin{array}{lll}8 & \text { Muro: } & \text { ok }+ \\ 9 & \text { hum sub chotte bachei hain [we are all little children] } \\ 10 & \begin{array}{l}\text { apko sir jukate hain, apne bojan diya } \\ \text { [I am bowing because you are giving me food] }\end{array} \\ 11 & \begin{array}{l}\text { aur sabhi kam swarpan karte hain, Amen! } \\ \text { [and you fulfil all of my wishes, Amen!] }\end{array} \\ 12 & \text { Ateesha: } & \text { thank you Muromaha that was nice }\end{array}$

Notably, Muromaha sounds linguistically insecure when she says grace in line 1 . Her voice is low and her words are not fully articulated. Because of the lack of clarity of the words, Ateesha has difficulty figuring out what has been said. Therefore, she addresses Sara with the question in line 2 asking what Muromaha has said. In turn, Sara requests a repetition in line 4 . The grouching sound in line 5, perhaps, voices Muromaha's discontent because of the repetition itself, or because she felt that she's being judged on a linguistic basis. However, Ateesha seems to take the latter interpretation and responds to it in a face-saving way (line 6), asking for repetition again in line 7 . In lines 9 through 11, Muromaha says the grace, produced as a rote learned phrase, perhaps learnt in the orphanage she was at in India (See Fogle, 2013 for orphan adoptees). In the post-recording feedback, the parents confirmed that Muromaha learnt to say this grace in the orphanage. It could be the case that Muromaha uses grouching to reject any discussion over her linguistic identity as a Hindi speaker again reject being 'othered'.

Overall, the home recordings showed that Muromaha's parents were highly committed to connecting Muromaha with her Indian identity, whether linguistic or otherwise. Yet, natural Hindi language use did not occur within the family which might be expected since the caregivers did not speak Hindi. Rather, it was limited to the use of isolated Hindi lexical items such as 'gaajaR' and 'khidhki:', as noted in Excerpts 6.6 and 6.7, and also a rehearsal of a religious formula (e.g. saying grace) as shown in Excerpt 6.8. This finding aligns with Kühl \& Peterson's (2018) who demonstrated that a limited collection of Danish lexical items remained in the repertoire of Danish community members in Utah. Yet, this (limited) use of Hindi made the home a Hindi-speaking space which in turn fostered Muromaha's sense of belonging to the family (Fogle, 2013). Adoptive parents often practice the children's heritage cultures rather than language (Shin, 2014) potentially due to their limited knowledge in the HL. Sara used 'bits of culture' (Blommaert, 2010), Indian music in particular in Excerpt 6.2, as an identity marker for Muromaha's Indian identity.

So far, Muromaha's family has exemplified a highly committed family with limited language proficiency. The following sections discuss the discursive identity practices within multiculturally committed and symbolically committed families. 


\subsection{Kevin's family}

Kevin left Fiji for New Zealand in his twenties and met Grace who also came to New Zealand from China at a similar age. They got married and had Havana, Austin and Max. Both parents worked full-time jobs, but they were multiculturally committed (See section 3.3). Kevin and Grace encouraged their children to learn HL literacy skills (Hindi, Mandarin and Cantonese) by attending community language schools and following up their progress at home. The family recorded around 120 minutes (two hours) of recordings, mostly during playtime. Below is a description of their practices.

\subsubsection{Elicitation of linguistic knowledge}

Kevin seemed to be responsible for keeping tabs on the children's language matters in the recordings. He appeared in the recordings several times asking his children to recall and 'recite' what they could remember in their HLs. Excerpt 6.9 is an example, taken from the recordings when Kevin asked Austin and Max to rehearse numbers in Hindi, which they had learnt in the HS.

Excerpt 6.9: Eliciting literacy competence in Hindi (numbers) (Home Recording)

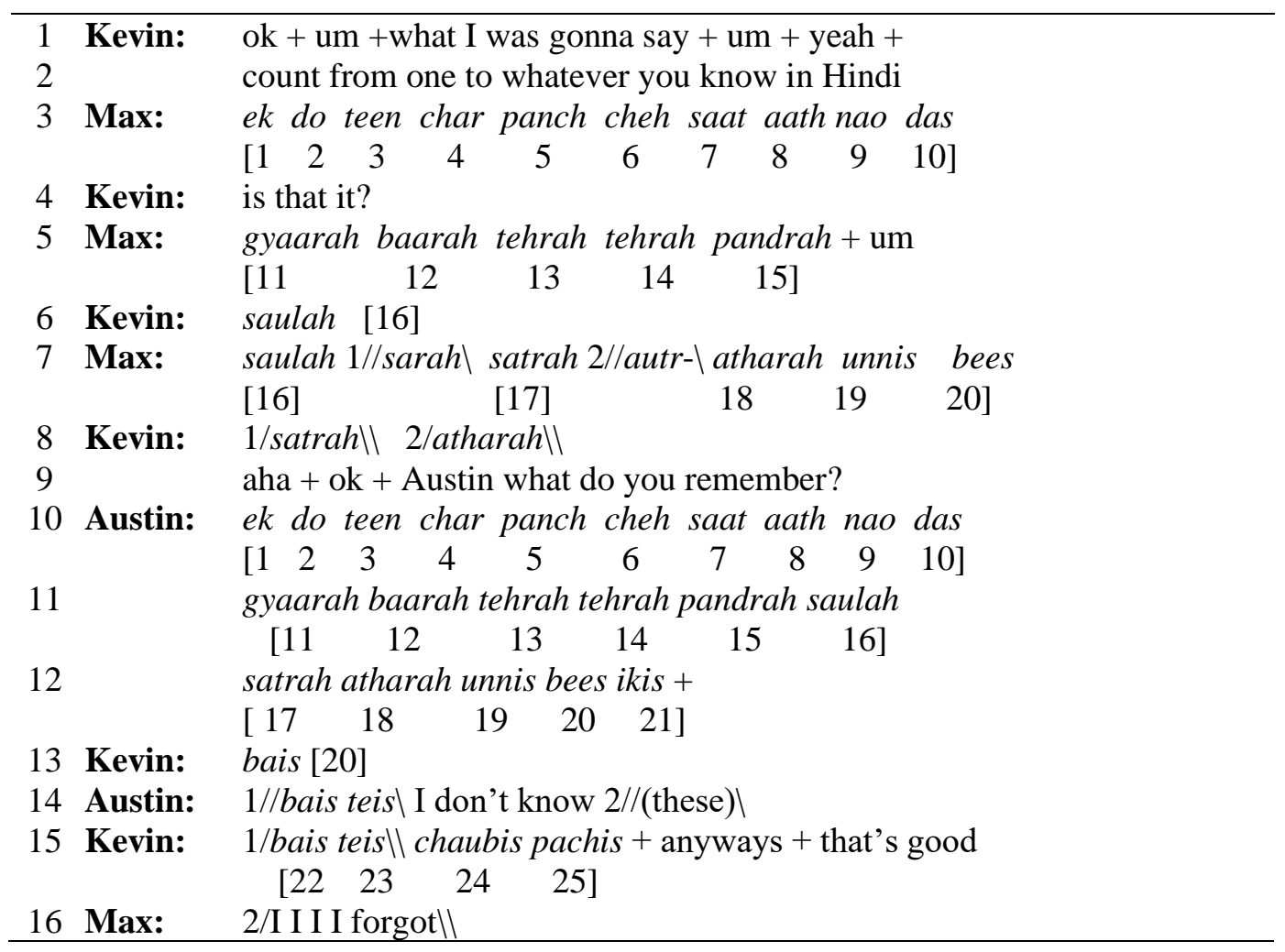

Kevin turns on the audio-recorder trying to engage with the children in a Hindi related conversation. Line 1 suggests that he pre-planned what he would like the recorder to capture. Kevin directly asks the children to count in Hindi, using the imperative form (line 2). In doing 
so, Kevin not only deliberately connects the children with Hindi through the literacy skills they have learnt in the HS, but also presumably wants to show his children as knowledgeable in their HL. Max responds to Kevin's request by counting to ten in line 3. The short list seems not to match Kevin's expectation and thus he encourages Max to continue counting (line 4). In line 9, the elicitation of Hindi continues when Kevin asks Austin to offer what he remembers, leading Austin to count to 21.

In the recordings, Kevin also elicited Hindi literary texts and formulaic expressions. An example of this is illustrated in Excerpt 6.10. The boys were playing video games when Kevin turned on the recorder and started asking them to 'recite' a poem.

Excerpt 6.10: Elicitation of literary texts and formulaic expressions (Home Recording)

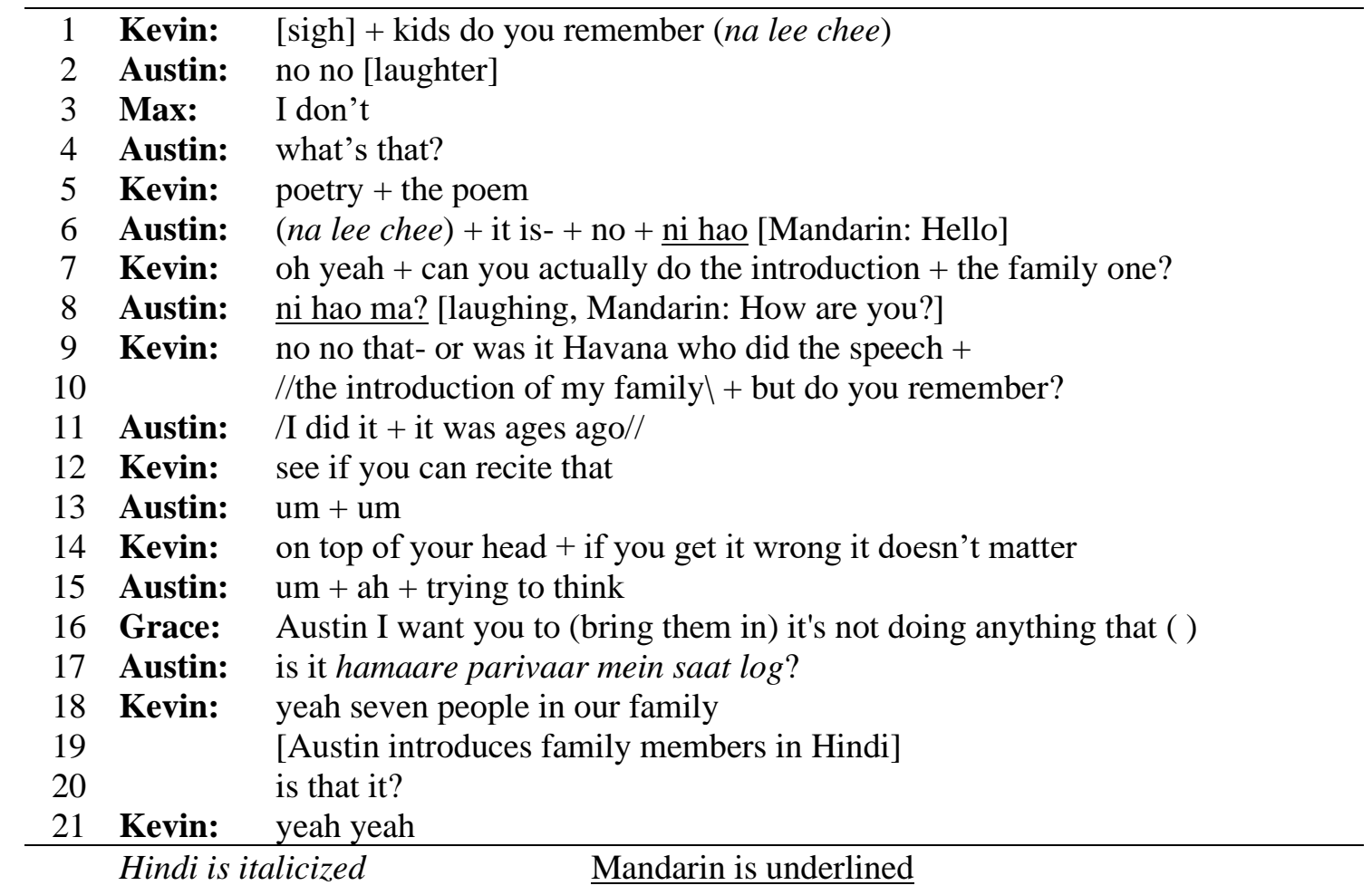

As noted in the Excerpt 6.10, Kevin asks the children whether they remember a poem they have learnt, but the boys do not know the poem. What's more, Austin seems not to recognise the language of the poem. When Kevin names the poem (line 1), the boys say they do not remember it. Austin asks for an explanation of what it is (line 5). Austin relies on his extended linguistic repertoire and seems not to be able to differentiate between his languages. He responds to Kevin's request by giving him one of his additional languages (i.e. Mandarin) saying ni hao which, perhaps, sounds like Kevin's phrase na lee chee (line 6), without noticing 
that Kevin is asking for a specific language ${ }^{7}$. Austin's response in Mandarin suggests that children in families with multiple languages might not differentiate between languages (See Lanza, 2004). It is worth mentioning that in the post-recording feedback Kevin confirmed that he was asking the children to recite a 'Hindi thing', although he could not remember the poem. In line 7, Kevin shifts from the rehearsal of the 'unknown' poem to the family introduction that the children have learnt in the HS, using a polite request. However, he receives an unexpected response ni hao ma? from Austin in line 8. The repetition of the Mandarin phrase ni hao ma? suggests that they need to socialise to use the language (Schieffelin \& Ochs, 1986) to negotiate the meaning of the introduction in lines 9 and 10. Kevin encourages Austin to remember his introduction of my family in Hindi (line 10) even if it is 'wrong' (line 14). The emphasis appears to be at this stage on the performance of linguistic competence rather than accurate language use. In line 17, Austin starts introducing the family although he seems hesitant. After getting positive feedback along with the English translation of what he has said (line 18), he recites the introduction in full. Hindi is thus arguably viewed as a school subject rather than a language to be used for communicative purposes. This corresponds to Lyutykh's (2011) finding that literacy competency was more important to Russian parents than language use. The purposeful rehearsal of linguistic competence in the HL was co-constructed by both Kevin and the children. Kevin's desire was for the children to be seen as engaging in Indian linguistic identity while the children cooperated by attempting to rehearse what they remembered.

Even though Kevin did not know Mandarin, he seemed invested in the children's literacy skills in Mandarin. Excerpt 6.11 is an instance where he seems to be attempting to elicit what the children know in Mandarin. The excerpt comes from a conversation at playtime.

Excerpt 6.11: Elicitation of Mandarin (Home Recording)

1 Kevin: now what- I mean is uh- what is it the- is it Mandarin you did at school?

2 Austin: Mandarin!

3 Kevin: you know? or was it Havana who did it?

4 Austin: Havana did some random speech

5 Kevin: oh Havana did!

6 Austin: I didn't really write it or speak $/ /() \backslash$ whenever she does something

7 Kevin: /here she's in ( ) 1 emm

Kevin is asking Austin about a Mandarin speech he thinks Austin did at school (line 1). However, Austin's exclamation suggests that he does not know the speech (line 2). Then Kevin

\footnotetext{
7 Another interpretation could be that Austin's use of the Mandarin formulaic phrases constructs a subversive response to what is being said.
} 
wonders if Havana was the speech giver. Seemingly he performs a face-saving act to avoid embarrassing Austin. Interestingly, Austin's response to Kevin's question Mandarin! is similar to the one in Excerpt 6.10, what's that?. These responses suggest that Kevin purposefully encourages the children to display their linguistic competence in their HLs.

Kevin arguably makes use of the presence of the recorder to echo their connection with the HS by using directives and polite requests to elicit the Hindi knowledge the children have learnt in the HS (See also Guardado, 2013), rather than using the language naturalistically, which in turn mirrors their membership in the HS as an emblem of their Indianness, as shown in Chapter Four. This finding is comparable with the finding of Ellis, Sims, and Knox (2018) that families of multilingual backgrounds expect their children to automatically acquire their HLs even if the parents use English with each other.

Having discussed the linguistic practices of Kevin's multicultural family, I turn now to a symbolically committed family, Kaya ji’s family.

\subsection{Kaya ji's family}

In this section, I describe the language practices in a multilingual Indian family, Kaya ji's family. I describe and analyse the language practices that connect them to their Indian heritage. As noted in Chapter Three, the family consisted of Peter, Kaya ji and two daughters, Ann and Mylu. Kaya ji's family enjoyed strong bonds with the grandparents. Since the parents worked full time, Ann and Mylu had daily contact with their grandparents after school, staying with them until their parents came home. Kaya ji's family was an Indian multilingual and multicultural family. As mentioned in section 3.3, Kaya ji was Fiji Indian, Peter was New Zealand-born to a Gujarati family and the grandparents (Peter's parents) were Gujarati and Hindi speakers.

\subsubsection{Food as a marker of identity}

While the family encouraged the girls to learn Hindi, the recordings gave evidence that the (grand)parents interacted with them in English. The interaction among members of the extended family is exemplified in Excerpt 6.12 in which the grandparents were present at the family dinner and engaged in a conversation about food including meta-linguistic commentary.

Excerpt 6.12: Food words signal Indianness (Home Recording)

$\begin{array}{lll}1 & \text { Grandma: } & \text { do you want some rice? } \\ 2 & \text { Mylu: } & \text { yeah } \\ 3 & \text { Grandpa: } & \text { they want (curry) and- } \\ 4 & \text { Kaya ji: } & \text { daddy's coming and bhaji's coming [vegetable snack] }\end{array}$




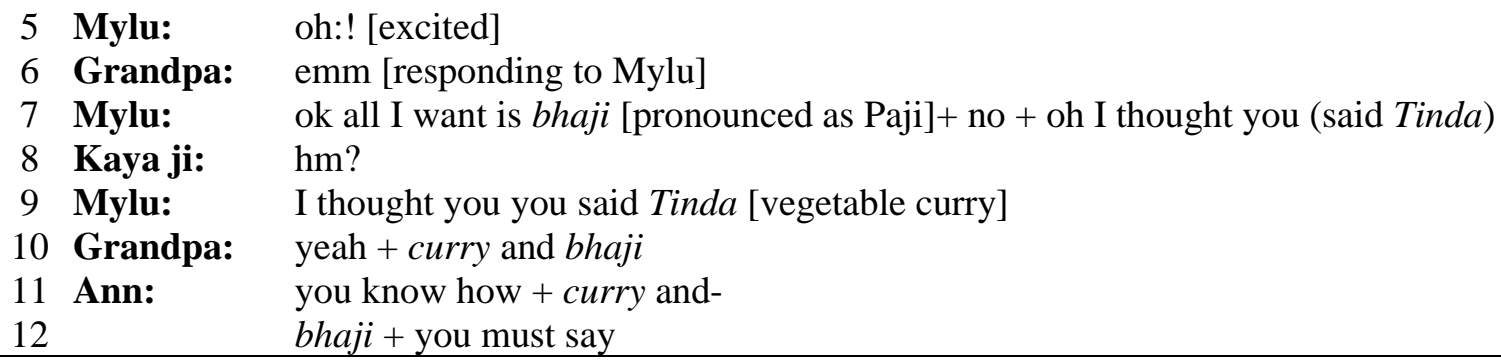

English is used as the main language in the interaction except some lexical items related to food such as 'bhaji', 'Tinda', and 'curry', all with Non-English pronunciation. While these isolated lexical items are viewed as codeswitching, they can serve as a stylistic choice that suggests acts of identity as well as performance and maintenance of that identity (Kühl \& Peterson, 2018, p. 225). Kaya ji uses 'bhaji' in line 4 to create a rhyme that offers a fun connection for the children 'daddy's coming and bhaji's coming'. Line 5 shows that Mylu's feeling of excitement echoes Kaya ji's success in establishing this connection. Furthermore, Ann's meta-linguistic comment on Mylu's pronunciation suggests that Ann recognises the word as a Hindi word. It is evident that Ann is performing an act of identity when she explicitly models to Mylu how 'bhaji' must be said (line 12). These examples offer evidence that the Indian identity forms an integral part of the lived experiences of the children at home. These lexical items are part of the children's everyday vocabulary, though limited to food. This, perhaps, leads to recognising their Indian identity and constructing it discursively. In this regard, Guzzo and Gallo (2019, p. 94) argue that talking about food is considered a social practice that offers significant insights into the processes of identity construction and heritage maintenance.

Seemingly, the connection to Hindi for this family is mostly found in language used for culturally important traditions and symbols (Robins \& Crystal, 2019). Hindi seems to be an emblem of the Indian identity and used for a limited collection of Hindi lexical items contingent on topics such as food. The relationship between food traditions and heritage culture is wellestablished (Gabaccia, 2000) and arguably food constitutes the most enduring connection with the past (Peterson, 2018, p. 80). Sometimes food words are the only remaining vocabulary items from the heritage language (Kühl \& Peterson, 2018). Using Indian food vocabulary is pervasive in the recordings. It seems that for this family all occurrences of Hindi at least in the utterances they produced are found in food talk. 


\subsubsection{Indian English in the home}

When considering language choice between parents and grandparents, it seems that English also dominated, as the following extracts show. The interaction between Kaya ji and the children's grandmother, as shown in Excerpt 6.13 was recorded when the family were at the dinner table.

Excerpt 6.13: Mother-grandparents interaction (Home Recording)

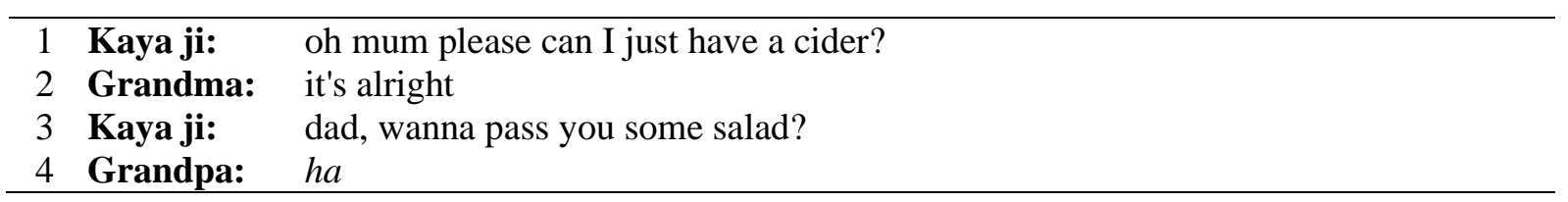

The excerpt shows that Kaya ji interacts with her parents-in-law in English. It is also evident that they use linguistic features associated with Indian English. In line 3, Kaya ji asks her father-in-law dad, wanna pass you some salad?, a structure in which the absence of subjectverb inversion in direct questions is frequently used in Indian English (Trudgill \& Hannah, 2002). Additionally, her father-in-law responds by saying $h a$, which is also associated with Indian English. In fact, the recordings included several instances of using the discourse marker $h a$ (Lange, 2012) with an even tone that derives originally from Hindi (Kachru, 2006). Excerpt 6.14 is another example where both Ann and Mylu used $h a$.

Excerpt 6.14: Using ha in interaction (Home Recording)

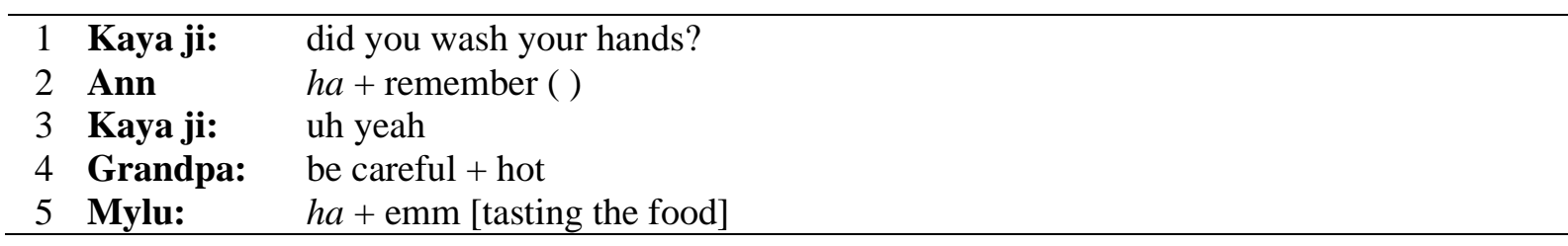

In the excerpt, Kaya ji asks Ann whether she has washed her hands (line 1) and Ann responds positively by saying $h a$ in line 2 . Again, the same pattern occurs in line 5 when the grandfather notifies Mylu that the food is hot, consequently, Mylu responds with ha. Using ha to respond to Kaya ji and Grandpa suggests that Ann and Mylu may be indexing their Indian identity by accommodating to the family's heritage, albeit through Indian English.

In the interview with Kaya ji, she reported that 'language stuff' was her responsibility because Peter did not speak Hindi. She also positioned Peter as a "Kiwi-English" speaker. The next interaction, Excerpt 6.15, shows the nature of the interaction between Peter and his daughters. The excerpt comes from a conversation before dinner time as the family was setting up. Ann and Mylu were watching TV while the parents were preparing dinner. 
Excerpt 6.15: Using Indian English and Indian food traditions (Home Recording)

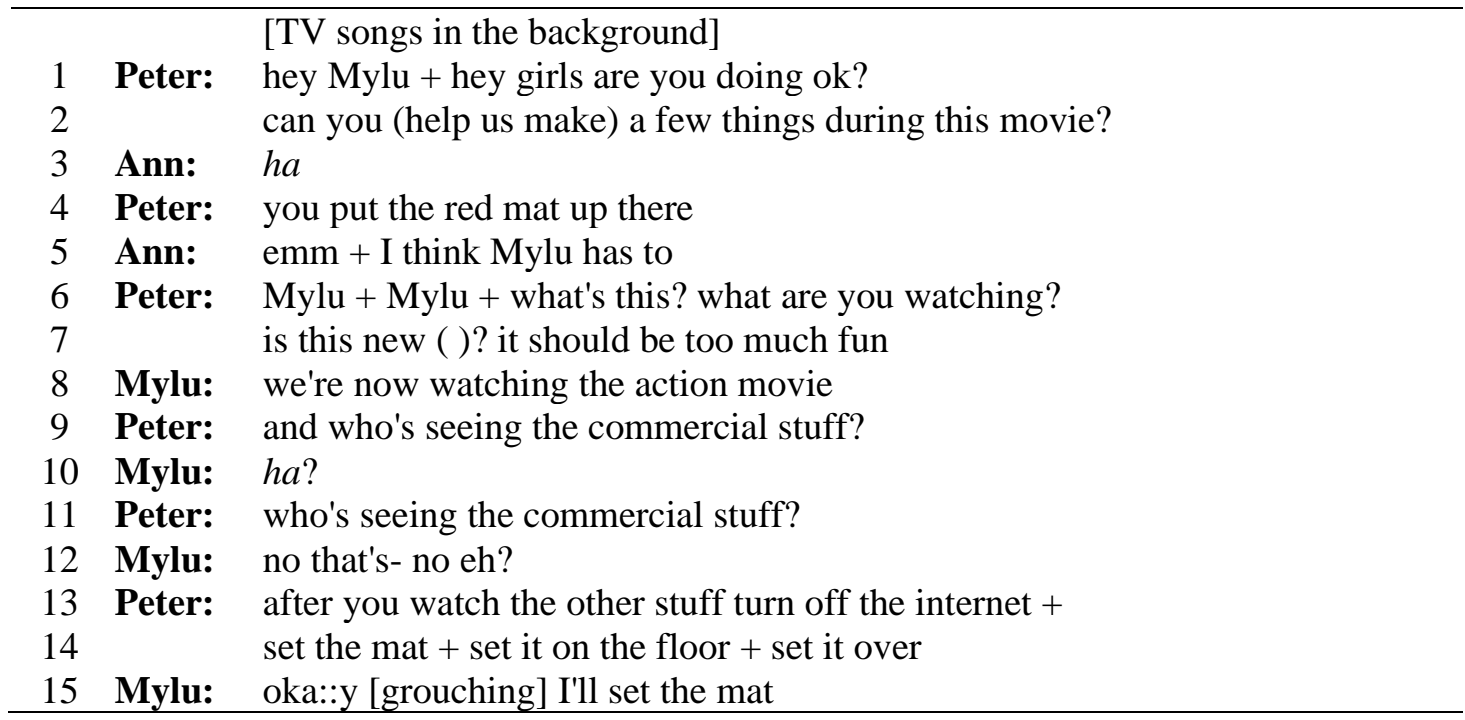

Peter asks the girls to help set up for dinner (line 2). In line 3, Ann agrees to help by saying $h a$, offering another example of frequent use of this linguistic feature associated with Indian English from within her linguistic repertoire (García, 2009). In line 4, Peter tells her to put the red mat (eating mat) over the floor. In line 9, Peter asks the girls another question. Mylu responds this time by saying $h a$ with an interrogative tone in line 10 as a way to elicit repetition. Noting that while it is the same form as $h a$ (line 3), it is serving a different function here ${ }^{8}$.

Excerpt 6.15 provides a strong example depicting Indian identity beyond language use. Although English is the main language in the home, traces of the family's Indianness are found in the use of features of Indian English such as ha. Seemingly Indian English embodies a symbol of culture and identity (O'reilly, 2003). The excerpt also shows the Indian lifestyle the family leads, especially Indian food habits. In lines 4 and 14, Peter asks the girls to set the eating mat on the floor in order to have dinner on it. In the same vein, Saunders (2007) demonstrates how a community of Hindu migrants in the USA use discourse on diet in their narratives as a way to emphasise connections with their community and India, in addition to constructing Indian, Hindu and caste identities. I argue that by using these features in the discourse, family members are actively engaged in giving meaning to what it means to be an Indian New Zealander by the way they socialise and eat to emphasise their connections their Indian identity.

\footnotetext{
${ }^{8}$ It could be that the choice of this particular form of clarification is related to huh/ha rather than eh?, what?, or pardon?, indexing Indian English.
} 
The recordings of this family showed that English was the dominant language in interactions among members within the immediate and extended families. However, it could be the case that the Indian identity was expressed by the variety of English used in this family, i.e. Indian English. The children were exposed to linguistic features associated with Indian English which were used in the discourse of Kaya ji, the grandparents, Ann and Mylu. Additionally, language use within the family, i.e. English, appeared to serve as a connection between language and the Indian identity which was expressed by their food discourse (Balirano \& Guzzo, 2019) and suggested that food was a marker of identity (Gerhardt, 2013; Lakoff, 2006).

\subsection{Conclusion}

In this chapter, I described and analysed the language practices of three families with different levels of commitment to HLs. Drawing upon home recordings in these families, the analysis revealed that no matter how committed community members were, the HL was not always actively used at home - a result that corresponds to findings in LM and HL research (e.g. De Bres, 2017; Revis, 2015; Shameem, 1995; Umali \& Bell, 2017).

The three families offered a wide range of language practices that indexed their Indian identity. The data suggested that the parents introduced aspects of the Indian culture, which were mostly indexed via music and food in the discourse (Friesen, 2008; Shah, 2013), e.g. to keep Muromaha connected with her Indian culture and identity, or at family dinner time for Kaya ji. Yet, sometimes this became problematic because it contradicted other socially constructed identities (e.g. being friendly) and membership in the family and school as it did in the Muromaha's family. Likewise, New Zealand cultural norms were presented in the form of the shared practices of Muromaha's primary school community.

Kevin's family encouraged the performance of the children's linguistic competence by eliciting HLs literacy skills such as numeracy and reciting of literary texts. The data demonstrated that Kevin's family was committed to connecting the children to Hindi and Mandarin. In addition to their active engagement with the HS, both Kevin and Grace took responsibility for their children's multilingual abilities. Kevin's family seemed to focus on HL literacy rather than language use. They appeared to engage with Hindi and Mandarin by eliciting literacy skills and rehearsing literary passages.

The data from Kaya ji's family showed that although the family was strongly connected to the extended family, English dominates the communication among family members. This finding was in line with other studies conducted with communities where such a strong bond with the 
extended family played an influential role in the development of a child's attachment to their heritage (Davis \& Starks, 2005; Umali \& Bell, 2017). This bond did not necessarily guarantee the use of the HL naturalistically. In this case, it could be due to the multilinguistic nature of the family where English was the lingua franca within the family. It is also worth considering that the grandparents were converging to their granddaughters as well as avoiding intrafamily tensions resulting from the language barrier between them and their grandchildren (Canagarajah, 2008). The analysis showed that this family used English to construct their Indian identity by using cultural lexical items as a marker of identity and linguistic features that were often associated with Indian English. These findings are discussed in the following chapter which brings together all my analysis, placing emphasis on the research questions. 


\section{Chapter Seven: Discussion}

\subsection{Introduction}

The preceding analysis chapters made use of data from three different sources (i.e. the school, individuals (children), and the home). The analyses focused on the language ideologies and practices at school and home, and although the families in the data set all viewed the HS as an overarching umbrella to connect their children to their Indian heritage, they adopted diverse language practices to enact Indian identity discursively. In this chapter, I draw upon the findings from the previous chapters to discuss the broad themes that emerged in analysis and to address the research questions. My research questions were:

RQ1: How does affiliation with the HL school connect HLLs to national/cultural identities?

RQ2: How do language practices in the HL school relate to multilingual identity development?

RQ3: How do HLLs interact with their linguistic and national/cultural identities in momentto-moment interaction?

In this chapter, I first discuss how the lived experiences within the HS connected the HLLs to an Indian national/cultural identity. I then move to give an account of how HLLs of Hindi made meaning of these experiences when developing multilingual Indian identities, followed by a discussion of their negotiation of linguistic and national/cultural identities reflecting multiple memberships. This chapter also highlights the implications of the thesis theoretically and methodologically. Finally I recognise some limitations of the research and note how they open up new possibilities for future research.

\subsection{The Indian national/cultural identity in the HS}

In discussing modes of belonging to communities, Wenger (1998b, p. 176) argued for the key value of imagination in shaping "[one's] experience of the world and [their] sense of place in it", which enables them to "[create] new images for the world and [themselves]". Wenger (1998b, p. 173) also pointed out that imagination facilitates "seeing connections through time and space by extrapolating from our own experiences". In this way, imagination lies at the heart of our identity construction; we have a feeling of belonging to a particular community and imagine that we share a common practice with other members. This community is often abstract and indirect because its "members will never know most of their fellow-members, meet them, or even hear of them, yet in the minds of each lives the image of their communion" (Anderson, 1991, p. 6). Imagined communities also suggest the presence of imagined identities 
which legitimise the individual's membership in the respective communities (Gao, 2012; Norton, 2001, 2013). In this thesis, I have captured the views held towards the HS as a space for the learners to gain a sense of belonging to a common Indian identity, arguably a connection to an imagined shared nationality/culture.

The parents in Chapter Four indicated that the HS constituted an institutional space that made up for their 'unsuccessful' family language policies. For example, Jeff and Kabir decided to bring their children to the HS because they felt their children would positively interact with the environment the HS offered. The parents viewed an institution like the HS as enabling families to get access to the imagined Indian community in which Hindi functioned as a heritage lingua franca. This access was facilitated by their membership in the HS CofPs in which they developed a shared practice. The HS community developed their shared practices including Discourse (Gee's (1996) Big-D Discourse) to build their Indian community as shown in Chapter Four. In doing so, they aligned themselves with the rest of their fellow members of Indian origin, even though those members did not know one another, nor even spoke the Hindi language, which they considered an emblem of the Indian identity.

Furthermore, the families attached to the HS constructed a superdiverse community in which members belonged to various linguistic, ethnic, religious backgrounds, but who were homogenized under the label 'Indian'. They had various national backgrounds: India, Fiji, South Africa and New Zealand. As shown in Chapter Four, this heterogeneous formation of the HS was recognised by its members and resulted in the salience of a Discourse of diversity which in turn influenced the practices within the school, e.g. the school language policy and planning. Despite such dynamism within the school, the participants displayed their sense of belonging to the wider imagined community of Indians in New Zealand. Their participation in the school practices, linguistic or cultural, signaled their investment in the imagined community (Darvin \& Norton, 2015) in which they located themselves and engaged with the world (Norton, 2001). Most importantly, their affiliation with the HS reflected their investment in the symbolic capital (Bourdieu, 1986) that they saw as associated to identifying with the HS rather than investing in actual language use. This was evident in their enrolment rates in the school and participation in Indian events, especially when compared to actual class attendance. It is important to note that while national identity was regularly viewed as fixed and determined by concrete geographical, familial, linguistic, and legal elements (Solano-Campos, 2015), and that the discursive construction of national identity was often fixed, stable and closed to change (Jensen, 2014; Suvarierol, 2012), members of the HS challenged these views by constructing 
national identity as multiple and mutable. The HS members' participation in nationally organised activities such as Hindi Divas and the Indian and Fijian Independence Days blurred the borderlines of the political underpinnings of Indian national identity. The participants conceptualised Indian identity as not having an immediate belonging to the Republic of India. Rather, Indian identity extended to include those whose ancestors migrated long ago and are no longer deemed citizens of India, those with 'hyphenated identities' (Caglar, 1997) such as Fiji-Indians. Such an expansion of the Indian national identity suggests the transforming of national identity to a catch-all national/cultural identity by the adoption of a pan-ethnic identity, i.e. Indianness, which in turn led to referring to Hindi as an Indian identity marker with which the participants identified to access to their imagined community.

As shown in Chapter Four, members of the HS and the communities around it provided evidence of the dynamic and complex nature of national identity; many of them were not citizens of India and had never visited the country. Yet, they identified as Indians and participated in Indian national events while simultaneously constructing their New Zealand national identity. This finding was supported by the HLLs' multilingual identities in their linguistic reflection drawings in Chapter Five. For example, Havana and Ann constructed their multilingual identities in a way that led them to negotiate the national and cultural identities as multiple, displaying belonging to both New Zealand and India. Lara also claimed Hindi and Spanish along with English and Telugu to construct her multilingual identity, leading to indexing her cultural identity as multiple.

Interestingly, despite the conception of Hindi as a 'heritage lingua franca' (Seals, 2013) in the HS, the language ideologies towards Hindi did not influence the actual language practices in the different HS CofPs. That is, English was still used to facilitate learning Hindi. Additionally, the HS located itself and efforts in the wider Indian community, but not just the Hindi speaking community due to the lack of institutional resources available for other Indian language communities, except the Gujaratis who had a well-established community and affiliated Gujarati school (Roberts, 1999). The observed, recorded and reported practices suggest that the HS efforts were targeted at the learners' attachment to an Indian identity in lieu of a specific linguistic identity. Although the school was designed to teach the Hindi language, a common Indian identity was more prominent than a Hindi linguistic identity in the HS. An exemplar of this was the teachers' participation in the off-topic discussions in class when the topic related to any of the Indian languages or other Indian cultural elements. 


\subsection{Multilingual identity development}

In order to examine the connection between the practices within the HS and the HLLs' multilingual identity development, there was a need to consider the language ideologies in the school. Language ideologies were primarily influenced by the diversity found in the HS. While the Discourse of diversity was salient and shifted the focus of the school members towards a common Indian identity rather than specifically a Hindi linguistic identity, the teachers and parents subscribed to the idea that Hindi as India's national language connectd the children to their Indian identity and heritage. They benefited from this ideology in their investment efforts since it provided them with an imagined community as a context for their investment (Norton, 2001) and also presupposed Hindi as a marker for a singular identity and a singular shared Indian heritage. This enabled them to "[maintain] and [secure] their identity only by eliminating the difference and otherness"(Benhabib, 1996, p. 4).

In terms of the learners' other languages, the teachers also demonstrated positive attitudes towards all the languages the learners knew. Such ideologies were reflected in the school language policy and practice as the teachers, for example, frequently asked the learners to offer native-language equivalents for the new Hindi vocabulary being taught. While the teachers positioned the children in the HS as Indians, and although there were times when they were called on as members of the Hindi community, the children were still positioned as Hindi learners rather than speakers. This positioning influenced the HLLs' self-positioning, as the analysis of their linguistic reflection drawings in Chapter Five revealed. Although most of the children identified themselves with Hindi and claimed a Hindi learner identity with various degrees of proficiency, they still showed strong connections to Hindi and gave Hindi high importance, similar to that of their HLs and English.

One of the features of multilingual identity development was the associative meanings the children assigned to Hindi. Drawing upon their experiences in the HS and the community at large, the HLLs associated Hindi with aspects of their lives. For example, Tattu and Lara did not have access to Hindi at home as their parents speak Telugu. However, Tattu's exposure to the language ideologies in the HS enabled her to reflect upon the experience she had with her grandmother and other people during her visit to India. Thus, Tattu decided, as noted in her description of the drawing, to invest in learning Hindi and then establish indexical ties for Hindi to build social capital (Bourdieu, 1986). Similarly, Lara incorporated Hindi in her linguistic repertoire because she felt that Hindi would help her to explore new experiences in the HS and the Indian community around her. In Ann's and Havana's cases, it was not surprising that they 
claimed Hindi because each of them had one Hindi-speaking parent, and although Hindi was not habitually used at home, both claimed to speak Hindi since they used it in the HS. Ann called Hindi her 'mother tongue' and connected it with the cultural capital she displayed when she referred to bits of the Indian culture in the Hindi-green connection. She also referred to Hindi as social capital by describing her Indian surrounding within the family and the HS. Havana, the oldest student in the HS, had developed strong identities regarding the languages she claims. Notably, Havana showed agency when she contested the way she was positioned and positioned herself as a Hindi speaker. Lucy was the only one who do not claim Hindi linguistic identity, which is not surprising because she was a newcomer at the time of data collection. If Lucy's position reflected how the HLLs viewed Hindi when they started attending the HS - a position where they had no rapport with the community and its language(s), Havana's strong identity in Hindi arguably embodies the outcome of participating in the HS for several years.

The language ideologies found in the HS were supportive of the learners' linguistic knowledge as noted in Chapter Four. These language ideologies seemed to influence the HLLs' claims to other languages such as Telugu, Tamil, Mandarin, Cantonese and Spanish. While my initial expectation was that the learners would claim linguistic identities as speakers of English and the HLs they used at home, they demonstrated that they developed identities as speakers of other languages as well (which were not used within the family). For instance, Havana and her brothers (Max and Austin) included four languages. Havana claimed Chinese (i.e. Mandarin and Cantonese) linguistic identity as a speaker since she had maternal ties with these languages and was learning them, without them being used at home. The children's other Indian heritage languages such as Telugu, Tamil and Kannada seemed disadvantaged because of the restricted institutional choices for the children to learn these languages. In this regard, Seals (2013) notes that the elimination of the Ukrainian language maintenance programme constrained UkrainianAmerican children's identity development. Similarly, the linguistic background of the participants in this thesis alludes to the status quo of Indian heritage languages in the HS. The limited availability of institutional choices for the Indian community languages, other than Hindi and Gujarati, redirected the families' language maintenance efforts towards a higher level of belonging, i.e. Indianness. This redirection of investment widened the scope of their access to multiple communities and could come at the expense of their HLs. In the following section, I discuss how my participants interacted with their linguistic and national/cultural identities as members of various overlapping communities. 


\subsection{Linguistic and national/cultural identities in multiple memberships}

The analysis of the HLLs' interaction with their linguistic and national/cultural identities requires reflection on their moment-to-moment interaction where they negotiate their various identities as they converse with their interlocutors. Thus, the home setting was a suitable place to explore the Hindi learners' patterns of identity work concerning their multiple memberships. In this section, I answer the third research question starting with the discursive identity construction found in each of the three families. I then discuss how the children in these families negotiated their linguistic and national/cultural identities in relation to the communities they belonged to.

In the previous analysis chapters, the HLLs in the HS constructed their membership in various communities, including but not limited to the family, the HS, the Indian communities, their public schools and the wider New Zealand society. In Muromaha's family, meta-discussions around her nationality and cultural values reflected how they positioned her based on her membership as Indian, as a family member and as a local. In contrast, linguistic metadiscussion suggested how she positioned herself linguistically as an 'expert' (Oliveira, Sadler, \& Suslak, 2007) who speaks 'normal Hindi'. Kevin's family displayed their connection to their Indian identity by the performance of linguistic competence, e.g. elicitation of literacy competence, literary texts and formulaic expressions in Hindi. Interestingly the same practices applied to Mandarin; Kevin appeared in the recordings to be eliciting the children's linguistic knowledge in Mandarin, showing their multilinguistic abilities in their HLs. Kaya ji's family constructed their Indian identity through food discourse (Balirano \& Guzzo, 2019) as a marker of identity (Gerhardt, 2013; Lakoff, 2006), accompanied by the use of linguistic features often associated with Indian English such as the use of 'ha' as a discourse marker. Through metalinguistic commentary on the pronunciation of food items (as when Ann commented on Mylu's pronunciation of curry and bhaji) and modelling the correct pronunciation in Excerpt 6.12, Ann used English to display a Hindi language expert identity (Leeman, Rabin, \& RománMendoza, 2011), which indexed her membership in the Indian community around her as well as New Zealand wider society.

While the three families enacted a range of acts of identity, they were more or less influenced by the children's multiple memberships. The way Muromaha interacted with her Hindi linguistic identity was different from that of her Indian national/cultural identity. On the one hand, Muromaha avoided, contested and refrained from contributing to the conversation to express disalignment with or rejection of her Indian identity when she felt that emphasizing 
her Indian identity interrupted her inclusion in the family (e.g. Excerpt 6.2). However, there were times when Muromaha constructed her national identity as Indian and simultaneously as a New Zealand local to show solidarity with her peers (e.g. Excerpt 6.3). On the other hand, Muromaha was happy to be positioned as a Hindi speaker by showing her Hindi linguistic capital within the settings where she rejected her Indian identity. This suggests that she did not feel linguistic identity interrupted her status in the family, whereas overemphasizing her Indian identity might do so. The analysis also suggested that Muromaha was more vulnerable than the other participants, and her feeling of being 'othered', however unintentionally, created a contradictory relationship between multiple memberships.

Unlike Murmaha, the children in the other two families did not seem to reject or avoid their Indian identities. This was evident in Kevin's family when Max and Austin aligned with Kevin's desire for them to display their linguistic competence in both Hindi and Mandarin (Excerpts 6.10 and 6.11). Furthermore, the family did not show strong engagement with the Indian identity due to the family's complex structure, as the father is a Fiji-Indian, the mother is Chinese, and their children are New Zealand-born. Instead, they identified as a multilingual, multicultural family keeping a balance between their multiple memberships in their heritage communities.

Similarly, in Kaya ji’s family, Ann and Mylu had access to many aspects of the Indian identity whether linguistic or cultural. While they claimed their linguistic identity as English speakers in Chapter Five, they (especially Ann) appeared to use Indian food lexical items, although in isolation, with special care given to the Hindi pronunciation (Excerpt 6.12). They also used linguistic features commonly associated with Indian English like the discourse marker 'ha' (Lange, 2012). Furthermore, they showed their Indian identity non-linguistically through food and the way they ate the food, for example, eating on a 'red mat' (Excerpt 6.15).

Overall, the children in the three families aligned with the way they were positioned whether as Hindi speakers (i.e. Muromaha) or learners (i.e. Max, Austin, Ann and Mylu). However, particular national/cultural identities were contested or rejected at times and were not always strongly constructed in language practice. This suggests that the HLLs in the HS challenged the political doctrine of 'one-nation-one language' because they did not equate linguistic identity with national/cultural identity. Instead, the HLLs claimed multiple linguistic identities including Hindi, Tamil and Telugu, among others, to negotiate their Indian identities. 


\subsection{Implications}

\subsubsection{Methodological implications}

One of my research contributions to the current scholarship is methodological. This includes the use of the ethnographic approach which enabled me to access the community as a legitimate peripheral member. My observations, the use of children's linguistic reflection drawings and the use of recordings of naturally occurring interactions in the home have brought further methodological rigour. In particular, incorporation of the linguistic reflection drawings and home recordings are more recent methods of data collection in the field of sociolinguistics in New Zealand, where data has traditionally been collected through surveys and questionnaires. Another methodological contribution is my use of Interactional Sociolinguistics (Gumperz, 1982) to understand how language is used in context. The combination of these data collection and analysis methods supports this kind of innovations which are beginning to emerge in LMLS.

My research is first and foremost grounded in an ethnographic approach. The rationale behind choosing this methodology lies in its appropriateness to investigating the dynamic and complex Indian communities attached to the Hindi School. It is also a powerful approach for collecting and interpreting authentic and contextualised language (Wilson, 2011). Moreover, it has helped me to gain a good rapport with children in a safe and secure environment, who might otherwise feel vulnerable. My observations in the HS and the community events created a more holistic picture of the dynamics of the community as well as their linguistic and semiotic practices. Furthermore, adopting the concept of Communities of Practice (Lave \& Wenger, 1991; Wenger, 1998b) allowed me to position myself a legitimate peripheral member. This positioning enabled me to access the insider-like understanding of levels of meanings throughout the interaction, but also gave me the ability to ask for further insights, exclusive to insiders, regarding practice and interaction. Additionally, the observational data helped me to decide on other methodological choices such as preparing appropriate and acceptable interview schedules and including the children's linguistic reflection drawings. The interviews with teachers and parents functioned as a source for validating and triangulating the observation data, but also provided deep insights into the different layers of meanings negotiated in the observed interaction. For example, the interviews with the teachers clarified the norms of assessing the students' behaviour as appropriate when my outsider identity as a teacher evaluated that behaviour as inappropriate. 
The linguistic reflection drawings were included due to the children's often-shifting identification during observations. Since collecting verbal data from children is considered challenging (Mauthner, 1997; Roberts-Holmes, 2018), this method facilitated the task of accessing their identification due to its suitability to the children's ages and interests (Seals, 2017b). At this age, 5-17, children often feel excited to draw and colour. The activity assisted them to express their thoughts about how they perceive themselves in relation to their languages while doing an activity they like. Recently, there has been a lot of interest in using drawings as a method of data collection from adults and children, e.g. language portraits (Krumm, 2004, 2013; Krumm \& Jenkins, 2001), reflective drawings (De Bres, 2019; Purkarthofer \& De Korne, 2019), and linguistic reflection drawings (Seals, 2017b). These methods proved to be useful for reaching a deeper understanding of how participants voice their experiences (e.g Melo-Pfeifer, 2017; Seals, 2017b) and embodiment (e.g. Busch, 2012; Coffey, 2015). Yet, there has not been research done through a lens of indexicality (Indexicality is discussed more in the theoretical implications). The innovative use of linguistic reflection drawings to explore the indexical meaning of the children's languages contributes to the field. The recordings of naturally occurring interactions at home offered in-depth insights into the complex formation of Indian families in New Zealand. While other communities are often seen as having linguistically homogenous families (e.g. Lyutykh, 2011; Revis, 2015), the home recordings provided evidence of the linguistic heterogeneity Indian families have even though both parents are Indians (e.g. Kaya ji's family). The home recordings, thus, captured some of the subtleties in participants' actual language practices and identity negotiations. Additionally, they functioned as a source of naturalistic data to triangulate the reported data from interviews regarding identification and language use.

\subsubsection{Theoretical implications}

This research also has theoretical contributions to make to LMLS research by investigating a superdiverse community. It contributes to the fields of HLE and FLP, and in particular to heritage language research in New Zealand. Sociolinguists have actively researched with various heritage language communities in New Zealand (Bell, Harlow, \& Starks, 2005; Holmes, 1993; Holmes et al., 1993; Revis, 2015; Seals, 2017a; Seals \& Olsen-Reeder, 2017; Shameem, 1995; Walker, 2011). While Hindi is the fourth most common language in New Zealand after English, Te Reo Māori and Samoan with over 66,000 speakers (Statistics New Zealand, 2013), the communities identified with Hindi are still under-researched. This thesis begins to fill this gap on Hindi and Indian communities in New Zealand. 
Additionally, this thesis contributes to LMLS research in the New Zealand context in the sense that it incorporates insights from the HL school and diverse homes. LMLS research has incorporated data from individual participants, homes, churches, clubs and gatherings, inter alia. Describing and analysing language practices in the HS and the home provides a better understanding of the interaction between these two environments. While my findings support Seals's (2013) argument regarding the influence of HL experiences on HLLs, they contradict the widespread view of the home as the most important domain for LM. Evidence of this was given by the interviewed parents who sent their children to the HS as they struggled to get them engaged with Hindi at home.

Similarly, this research contributes to the field of family language policy by including a transnational adoptive family - an area in which linguistic research remains scarce (Fogle, 2012). This promotes a better understanding of what family interaction and dynamics might look like beyond stereotypes. This thesis also brings about insights into 'cultural socialisation' (Shin, 2013) as a developing area of the field, which is not habitually covered in HL research in New Zealand. In fact, there is no known research of adoptive families in the New Zealand context. Therefore, this thesis sheds some light on the understanding of the complexity of LM patterns due to the heterogeneous family configuration found in the recruited families in this research. Furthermore, this study provides evidence that family language policy needs to expand from focusing on the language per se to negotiating meaning within the family and engaging in being a family, as King (2016) suggested, through a 'discourse of belonging'.

In heritage language education research, this thesis provides significant insights into the application of the concept of CofPs. By describing and analysing the practices within the HS, the findings of this thesis challenge the pervasive understanding of the CofP concept in Heritage language education research. While HL communities are traditionally treated as 'heritage communities of practice' (Adell et al., 2015), my findings have highlighted the complexity of the communities around the HS. These communities are diverse nationally, ethnically and linguistically and are much closer to 'imagined communities' (Anderson, 1991) in engagement, as they are symbolically connected to each other through India's national language, Hindi. This connection is constructed through their high investment in identifying with the HS and its activities, but not necessarily in language learning. Similarly, reviewing their heritage language education provides insights into the applications of CofPs as fixed when examining language maintenance schools (e.g. Lyutykh, 2011; Oriyama, 2016). The Hindi School is an illustrative example of heterogeneous HL institutional spaces, in that it 
exerts tremendous effort to support the Indian families in New Zealand in their endeavours to stay connected to their Indian heritage and identity through the weekend language maintenance classes. By describing the practices in the three HS sites, the findings of Chapter Four identified the lack of one 'community' criterion, that is 'mutual engagement' (Wenger, 1998b), which in turn contributes to the distinct practices extant in each site. This thesis suggests that the CofP might not be the appropriate concept to represent heritage language schools without careful reflection. Rather, researchers are advised to bear in mind the usefulness of the mutual engagement criterion in creating a localised meaning within a studied group. In this case I argue that a 'constellation of communities of practice' (Wenger, 1998b) is a better conceptualization, whereby different CofPs are connected to each other in terms of their joint enterprise and shared practice.

In addition, I applied Ochs's (1993) concept of indexicality in the heritage language research to identify the semiotic links the HLLs create between their languages and social meanings (Bucholtz \& Hall, 2005; Ochs, 1992). To my knowledge, this represents the first application of this theory with young participants in the field. Ochs (1993) prioritises direct-indirect indexicalities. My analysis identified the (more and less) direct indexicality in the children's data in which they depicted language, but not a specific labelled language (García \& Li, 2014), as a tool for communicative purposes and associated with different forms of 'capital' (Bourdieu, 1986). This was exemplified by associating Hindi with social capital (Tattu) and cultural capital (Ann). These semiotic links were dependent on the interactional context for their meaning (Bucholtz \& Hall, 2010, p. 21). The use of colours in the drawing task helped to reshape the interactional context into an exciting and joyful context rather than a 'normal' conversation which younger participants might not tolerate. Overall, these findings support the current view of language as a linguistic system comprising the entire linguistic repertoire an individual has and can be accessed as they need it (García, 2009). Moreover, the analysis identified a range of 'indirect' indexicalities in the participants' engagement and development with previously created semiotic links, i.e. direct indices, to generate more abstract meanings, including the construction of multilingual identities and national/cultural identities.

Last but not least, this research contributes to language and identity research. The analysis of language practices at home has demonstrated the significance of multiple memberships for identity construction and negotiation. The home data provides insights into how the families engage with multiple memberships. While language is taken as "our primary semiotic tool for representing and negotiating social reality, including social identity categories" (Bailey, 2007, 
p. 341), my participants displayed and negotiated their heritage identities primarily through meta-discourse. My findings also support other findings, including that speaking the HL is sometimes the only means to display various HL identities, whether ethnic, national or cultural (Edwards, 2010; Ngaha, 2005, 2011).

Additionally, the multilingual characteristics of the Indian community adds more value to this research. Exploring identity negotiation in an Indian group contributes insights into the study of language identity since this group, to the best of my knowledge, has not received enough attention, especially in the New Zealand context. The diverse nature of the HS provides insights into how the HL school accommodates its language practices in accordance with its members. This finding reflects the HLLs' agency in language socialisation (Revis, 2015) where children act as socialising agents (Luykx, 2005). This was evident when the teachers socialised the children in English and acknowledged the learners' languages. The flexible school language policy demonstrates how the focus shifts from linguistic identity to a panethnic identity to provide an all-inclusive space. This suggests that the diversity in the HS has a strong impact on how the teachers position the HLLs. Having children from various Indian linguistic backgrounds allowed for positioning them as learners of Hindi and speakers of their Indian HLs, which in turn influenced the children's identity connections with their languages, as Seals (2017b) suggests. As shown in Chapter Five, the children included Hindi in their linguistic repertoires claiming their linguistic identities as Hindi learners, and this was reflected in Chapter Six when no actual Hindi language use was found in the recordings of the three participating families. Instead, they resorted to enacting their pan-ethnic identity, i.e. Indianness. This relates to the continuity of their investment in the language they might (not) feel committed to. Such a finding corresponds to other findings in the literature. MenardWarwick (2007) argues that Spanish HL students' language development is connected to how they are positioned in the HL school. Seals (2013) also notes the influence of how Russian HL students are positioned within the classroom on their identity connections with their heritage languages.

The findings, especially from Muromaha's family, highlight her response to being positioned linguistically and nationally/culturally. While she resisted and rejected being positioned as Indian in the family context, she accepted being positioned as an expert Hindi speaker. This connects to the language expert identity (Leeman et al., 2011) that Muromaha and Ann performed in interaction at home. 
Having presented the contributions of this research to language maintenance and language shift, family language policy, heritage language education, and language and identity research, I acknowledge its challenges and limitations in the following section.

\subsection{Limitations}

Although this research has contributed to various fields of knowledge, some limitations should be noted. First, this ethnographic research took place in a three-site Hindi school over one academic term. The three school sites held weekend classes with two of them having classes at the same time (Sundays 10 am to mid-day). This constrained the observations. Thus, I had to schedule my visits to these sites so that I could observe as many as possible of the classes in a balanced way. While this minimized the amount of data observed at each school site, it provided a holistic picture of the practices in the HS at large and provided me with a larger participant pool.

Second, the findings in this research are restricted to the time, location and resources available at the time of the study. The analyses provide a profile of the complex nature of the Indian families attached to the HS, and suggests that the demographics of the Indian community have dramatically changed from a Gujarati majority of the past to a wide range of backgrounds of Hindi speakers, including Fiji-Indians (cf. Roberts, 1999). This suggests that the current results are subject to change as a result of demographic changes. However, this thesis has offered insights into what it means to be an HL community member in a superdiverse country like New Zealand. In addition to that, if I had included other Hindi schools in different cities such as Auckland and Christchurch, I might have obtained relatively wider patterns but without the depth I could capture at this HS. Regarding resources, I here refer to the resources that the HS had during the ethnography. For example, there was a lack of facilities. The venues where the Hindi classes took place were not consistently reserved for the HS. Sometimes, the teachers had to move the class to, for example, a nearby mall, because the space they were using was used as a polling station (Fieldnotes 23 September, 2017). Sometimes, they had to merge classes as one of the rooms they normally used was booked by another activity (Fieldnotes 3 September, 2017). This caused disturbance during the observations because when the settings changed the practices changed. Another issue was my ability to note or record the practices when the classroom became crowded. However, I tried to moderate this challenge by extending the observation. 
Third, using linguistic reflection drawings was challenging and rewarding. Previous studies note some challenges in using drawings in terms of interpretation and implementation (e.g. Kalaja, Dufva, Alanen, \& Barkhuizen, 2013; Melo-Pfeifer, 2015; Melo-Pfeifer \& Schmidt, 2012). One challenge is related to the subjective interpretation of drawings. It was difficult to ensure objective interpretations of the children's choices due to their often-changing decisions. Some children in this research changed the colours many times, and others changed the colourlanguage association. While this made the task challenging, making the drawing task a conversational encounter rather than unidirectional from the children's side helped moderate this challenge. Moreover, the children in this study seemed to have different interpretations of the instructions given to them regarding the drawing task. Yet, because I was aware of this challenge due to my experience with children as a teacher, I tried my best to temper this by giving them one very specific instruction at a time to ensure their interpretations of the instructions were as I intended. Despite these challenges, linguistic reflection drawings proved to be helpful to the study of identity negotiations when paired with other data collection methods such as observations and recordings of naturally occurring interaction for triangulation purposes. The use of linguistic reflection drawings also offers a better understanding of meaning-making as well as relating the participants' social reality to their language ideologies.

Other challenges are related to the participants. Like other multi-step research studies, there was participant attrition when I requested that the participants take part in an additional task. For example, some parents whose children participated in the observation did not want to be involved in the drawings nor the home recordings. Participant attrition limited my ability to follow up the dynamics of some children's multiple self-identifications to verify the observed data, which also led to limiting the exploration of the dynamics of the community of study.

In addition to methodological challenges, throughout this research, I experienced challenges related to my status. At the beginning of the research, there was a list of things to consider before data collection. In addition to the literature and deciding on the research design and methods, I have had a 'to-do' list relating to fieldwork. Bowern $(2015$, p. 37) proposes that researchers should think of where, how, what data and from whom the data would be collected. While Bowern's list seemed more related to language documentation, it helped me to reflect upon many issues including my position as (1) new to the country, (2) an 'outsider' who belongs to a different culture, and (3) non-speaker of the heritage languages used within the community I would be working with. These points encouraged me to commit to the question 
of how to connect with the Hindi community, how and where to collect the data, and how to manage the data. These questions also helped me to get a nuanced picture of the challenges I would be facing.

Likewise, I bore in mind that many scholars (e.g. Abbi, 2001; Macaulay, 2011) discuss identity changes and losses that researchers may undergo while in the field. I align with the viewpoint that "a good fieldworker doesn't lose their identity, but a good fieldworker doesn't remain unchanged by their experiences either" (Bowern, 2015, p. 14). I did not feel that cultural differences between the community I would be working with and my own culture would negatively affect my researcher identity. Since it was not the first time I have researched with an Indian community, cultural differences and similarities remained on my radar. My past research experience with Indian communities dates back to my MA research, which I conducted in Yemen (Nofal, 2011). Additionally, since my arrival in Wellington in 2016, I have made friends who belong to the local Indian community. In turn, this helped me access the community. In other words, having friends who speak the language helped me understand the community and created opportunities for the Hindi speech to be transcribed and translated by native speakers when necessary.

As time passed, my position dramatically changed from being an outsider to a member on the periphery as I was not entitled to fully participate in the negotiation. Yet, members of the school community referred to me as an expert linguist who was able to give credible advice. This expert identity was constructed by both parents and teachers. Teachers referred to me when answering language learning related questions to support their answers to the children's questions. For instance, Deepika ji in Chapter Four (Excerpt 4.6) was asked by Raj about India's Māori name. While discussing India's names in different languages, Deepika ji noted that names of countries do not vary across language and asked me for confirmation. The parents also positioned me as an expert when we had informal conversations. On occasion, I chatted with Kabir and asked him about his language policy with his children. He said that he would tell me providing that I give him advice on how he could manage their language use.

These identity changes and challenges were rewarding as they gave me an insider-like status that enabled me to take part in the school practices as well as the privilege of inquiring about further insights. 


\subsection{Conclusion}

In this chapter, I discussed the themes that emerged in the previous analysis chapters in light of the existing literature. Specifically, by looking at the influence of the heterogeneous structure of the school on practices and the dominant ideologies at the school and the children's HLs, this chapter highlighted the connections that the participants establish with national/cultural identities through their membership in the HL school. It also explored the relationship between HL practices and the young participants' multilingual identity development through an indexical lens and the ways in which these participants interact with their linguistic and national/cultural identities in interactional encounters in the home. Reflecting on my findings and experience, I noted the contribution of this research to the field of heritage language education and LMLS more generally. In particular, I highlighted methodological contributions such as the use of an ethnographic approach, linguistic reflection drawings and recording interaction at home. I also showed how this thesis addresses some of the existing gaps in LM and HL literature internationally and in the New Zealand context.

In the next and final chapter, I summarize the research findings and reflect on directions for future research. 


\section{Chapter Eight: Conclusion}

\subsection{Introduction}

The relationship between sociocultural realities in migrant communities and identity negotiation and development is dynamic. This thesis investigated how the lived experiences of young multilingual heritage language learners align with their identity construction and development. At the time of data collection, the participants were attached to a Wellington community-based Hindi language maintenance school and had a range of Indian ethnic, social and linguistic backgrounds. The school's overt mission, as stipulated on the school website, was to connect the students and their families with their Indian heritage and to contribute to the maintenance of Hindi language as their mother tongue. This made the HS a valuable site for undertaking ethnographic research to explore the learners' affiliation with the HS and how this relates to their identities. In particular, researching the HL students' perception of their 'selves' and languages illuminated the understanding of the vitality of their participation in the HS in identity construction.

As noted in Chapter Two, while this thesis is located within LMLS research, it focuses on and highlights the relationship between HL and identity. Language and identity are inextricable; social actors use language to enact, resist and negotiate identities (Bucholtz \& Hall, 2005; De Fina et al., 2006; Norton, 2000). Chapter Two identified gaps in the literature locally and internationally, leading to research questions in accordance with the goal of this thesis.

Chapter Three operationalised the research questions by discussing my methodological choices and the procedures I followed to explore the language practices in the Hindi School and the home, in each case with a touch on the heritage language learner's identity negotiation and development. By articulating the rationale behind using an ethnographic approach, this chapter added weight to the argument regarding the suitability of linguistic ethnography in HL and identity research.

By drawing upon extensive ethnographic data from observations and interviews in the school, the heritage learners' linguistic reflection drawings, and naturally occurring interaction in the home, Chapters Four, Five and Six form the analysis section of the thesis. Chapter Four focused on the practices at the HS as an institutional space for HLs to facilitate an exploration of how the participants' affiliation with the heritage language school connects them to their Indian identities. The concept of 'communities of practice' (Lave \& Wenger, 1991; Wenger, 1998b; Wenger et al., 2002) provided the analytical tool for working with the observed and recorded 
practices at the school. By analysing the school as a "constellation of CofPs" level rather than the "CofP" level, the analysis offered an enriched perspective on the understanding of the heterogeneity of heritage language schools, challenging the fixed view that heritage language schools are homogeneous. Chapter Five sought to demonstrate how the heritage language school practices connect with the heritage language learners' multilingual identity negotiation and development. It also highlighted their language ideologies towards their languages. Analysing the HLLs' linguistic reflection drawings (Seals, 2017b) along with their descriptive accounts provided insights on the 'indexicality' (Ochs, 1993) found in the learners' discourse, of which Bourdieu's (1986) various forms of capital held considerable value. Chapter Six explored the family language practices in order to get a nuanced picture of how the heritage language learners discursively interact with their national/cultural identities. Adopting Interactional Sociolinguistics (Gumperz, 1982) when analysing the recorded data in three families provided detail about how these families construct and negotiate their Indian identity. Chapter Seven answered the research questions and outlined the implications and limitations of this study. This chapter provides a brief overview of the thesis and offers a summary of the research findings. It also suggests directions for future research. It concludes with my final reflections on this research as an experience I lived over the last few years.

\subsection{Summary of research findings}

The purpose of this study was to explore identity negotiation and development of young multilingual heritage language learners in a multi-site New Zealand community language school. Three research questions guided the study. While two of them (RQs 1 and 3 ) identified the language practices in the HL school and at home, RQ2 sought to delve into the children's multilingual identity development. To answer the proposed research questions, I adopted an ethnographic approach to mobilise rich data in two dynamic spaces, i.e. the HL school and home. I first described and analysed the school data in Chapter Four, and then the HLLs' selfidentification data was analysed in Chapter Five. Then, I analysed the home data in Chapter Six.

In answering RQ1, "How does affiliation with the HL school connect HLLs to national/cultural identities?", I collected observed and reported data. I conducted observations in the three school sites over the school term to gain a holistic picture of the practices within the school. I also conducted eight interviews with parents and stakeholders in the school to verify the observed practices and gain insights into those language practices and ideologies that 
contribute to national/cultural identities. By adopting the concept of communities of practice (Lave \& Wenger, 1991; Wenger, 1998b; Wenger et al., 2002) as an analytical tool, the analysis identified the participants' language ideologies and practices within the HS. The teachers acknowledged the students' languages and placed a high value upon the students' multilingual abilities. This included English, heritage languages and other languages (e.g. Te Reo and Spanish). Both teachers and parents accorded high value to Hindi as an emblem of the collective Indian identity, likely due to its status as the official language of India. The dominant language ideologies were influenced by the diverse configuration of the school, which in turn directed the school practices to be all-inclusive and homogenize its members under the label 'Indian'. The parents emphasized the role of Hindi and the HS in connecting their children with their Indian heritage. They displayed their belonging to a wider imagined community of Indians in New Zealand through their participation in school practices, whether linguistic or cultural. That is, parents viewed their (children's) membership in the HS as a sign of their Indian identity. They invested in the symbolic capital (Bourdieu, 1986) that they saw associated with the HS by identifying with the school rather than investing in actual language use.

The students' membership in the HS contributed to shaping their language ideologies towards Hindi as the 'heritage lingua franca' (Seals, 2013). These language ideologies were instrumental in the students' construction of multilingual identities as bi- and multilingual speakers in an Indian community, as noted in Chapter Five. Finally, The language practices in the HS revealed that both teachers and students used discursive practices to create localised meanings for interaction. For example, the evaluative discourse regarding how to greet who and what topic is relevant to classroom talk derived from recognisable Indian cultural norms.

To answer RQ2, "How do language practices in the HL school relate to multilingual identity development?", students' linguistic reflection drawings were paired with their detailed descriptive accounts of their decisions and choices to investigate the HLLs' dynamic and complex self-identification as well as the perceived relationship between the HL practices and their multilingual identity development. The analysis of the HL students' data drew upon Ochs's (1993) indexicalities and revealed that the student's language ideologies were parallel to those held in the HS. The students claimed their Hindi linguistic identities as learners rather than speakers. While Hindi is connected to the HS as a space for language use, it still enjoys equal importance to that of their HLs and English. The findings indicated that the students drew upon their linguistic and social realities in the HS and the imagined community of Indians to connect Hindi to their lives. The inclusion of Hindi in their linguistic repertoires and the 
development of associative meanings related to Hindi signalled the development of new Hindi linguistic identities that they can add to their linguistic repertoires.

The analysis also showed that the children used their languages as indices to display forms of capital (Bourdieu, 1986), which in turn contributed to their identity negotiation. The children showed that they developed strong linguistic identities regarding the languages they claimed. They identified themselves as speakers of their HLs and English. While the HLs were associated with linguistic and cultural capital and were indexically tied to Indian cultural identities, English was associated with linguistic, social and cultural capitals indexing a New Zealand national, and sometimes cultural, identity. The children typically identified themselves as Hindi learners with multilingual identities, and paired their Hindi linguistic identities with their experiences in the HS and the Indian community more widely. They also used Hindi as social and cultural capital to index an Indian national identity. In this way, the children indexed national and cultural identities as multiple by negotiating their multilinguistic identities.

RQ3 focused on the HLLs' interaction with their linguistic and national/cultural identities in everyday conversations within the home: "How do HLLs interact with their linguistic and national/cultural identities in moment-to-moment interaction?". To answer this question, naturally occurring interactions in three households were recorded and analysed and language practices were identified. The analysis suggested that parents used several language practices as acts of identity (Le Page \& Tabouret-Keller, 1985). Muromaha's family, an adoptive family, made use of metadiscourse to discuss aspects of her Indian heritage with Muromaha. These meta discussions foregrounded Muromaha's linguistic, national and cultural identities. While Muromaha accepted her linguistic identity as a Hindi speaker and built upon this to claim an 'expert' Hindi linguistic identity (Leeman et al., 2011), she resisted, avoided and rejected being positioned as Indian at times when she felt that her Indian national/cultural identities would interrupt her membership in other communities (e.g. the family).

These kinds of deliberate displays of linguistic identity were salient in the data. This is illustrated by Kevin's attempts to elicit the children's linguistic knowledge in their HLs (Hindi and Mandarin) with which the children aligned by responding to his requests. Finally, language practices in the home also included using food discourse (Balirano \& Guzzo, 2019) as a marker of identity (Gerhardt, 2013; Lakoff, 2006) which allowed the children to enact linguistic and national/cultural identities. While Muromaha had previously avoided her Indian identity, she accepted being positioned as an expert in the area of Indian food and recounted her experiences in India. In Kaya ji's family, Ann used food lexical items to show an expert Hindi linguistic 
identity when she taught her sister Mylu how curry and bhaji 'must' be pronounced. This family also used linguistic features commonly associated with Indian English like the discourse marker 'ha' (Lange, 2012) to enact Indian identity.

Overall, the analysis revealed that the participants' multiple memberships played a key role in the way they constructed and responded to their linguistic and national/cultural identities. While the parents drew upon various language practices for identity construction and cultural preservation, the younger participants showed variation in the ways they responded to these identities. Most of the children accepted and aligned with a prescribed-by-others HL linguistic identity, however, the Indian national/cultural identities were either contested or not salient in the interaction.

\subsection{Future directions for research}

This research has offered insights into the dynamic and complex nature of identity negotiation and development amongst learners of Hindi as a heritage language. Based on the understandings from the findings of this study, there are several recommendations for future research. First, this study has focused on a multi-site community language school in the greater city of Wellington, New Zealand. This creates opportunities for researchers to conduct future research in other community language schools in other cities such as Auckland and Christchurch for comparison purposes and to highlight any potential regional subtleties. Also, because the participating families in this study were multicultural in the sense that there was only one or no caregiver who speaks Hindi, it would be useful for researchers to conduct similar research looking at Hindi speaking families to get a better understanding of their language practices and LM efforts.

The findings have raised awareness of the need for investigating less commonly taught (Indian) languages in New Zealand. Some of the young participants in this research reported taking Tamil and Telugu classes. This suggests the need for researching linguistic identity development among heritage language learners of Tamil and Telugu (among others). Similarly, since heritage language research with adoptive families is scarce locally and internationally, future research might be able to reach a better understanding of the interplay between language, identity and their multiple memberships.

Finally, the methodology used in this research proved to be of great value in investigating macro and micro levels of identity construction and development. While the observed and reported data pinpointed the dominant Discourses (Gee, 1996) and practices in the HL school 
communities, recordings of everyday language revealed that membership in various communities whether immediate or imagined is a key factor in identity negotiation. I hope this thesis will encourage researchers to use similar methods in which macro and micro analyses are implemented in order to study other heritage lingua francas in New Zealand such as Arabic and Spanish, ideally, research in which investigators reconsider the automatic assignment of communities of practice to heritage language schools. By doing so, we can ensure that community members achieve the 'mutual engagement' criteria, and if not, a different, more appropriate model should be considered.

Most importantly, as the first study in New Zealand to investigate identity construction and development among heritage language learners through an indexical lens, I have laid the groundwork for future research in this area. As mentioned in the limitations, the length of observations in this research was not long enough to understand the indexical orders (Silverstein, 2003) of the children's descriptions of the drawings. Future research will hopefully address this need.

\subsection{Final thoughts}

This study has advanced an understanding of the dynamics and heterogeneity of heritage language communities and the various ways community members negotiate and develop their identities. When I first engaged in this project, my intention was to address a community that had not received much attention in linguistic research. After having engaged with their activities and practices, I became very aware of how profoundly they strive to maintain their heritage languages and identities and their desire to gain official recognition of Hindi in public schools. This brought about a change in my perspective from looking for descriptive data of their practices to putting myself in their shoes and feeling just how hard it is when we see our identity fading away. My stance has also changed accordingly, from being with them for research purposes to being a friend of the community whose duty it is to contribute to their endeavour. Thus, I actively participate in their events, and continue to lend them a hand either physically or intellectually. I have delivered speeches on the importance of maintaining heritage languages. I have also contributed to the Hindi School magazine 'Darpan' by writing an article on the benefits of multilingualism.

To me, the most important finding in this thesis is the community members' views of the key role of heritage language education in supporting family language policy and linguistic identity development. Therefore, I hope the Ministry of Education will take this study into account 
when making the decision to include Hindi, and other heritage languages, in New Zealand schools. 


\section{References}

'Aipolo, A., \& Holmes, J. (1990). The Wellington Tongan community: Prospects for language maintenance. Wellington Working Papers in Linguistics, 1, 1-16.

Adell, N., Bendix, R. F., Bortolotto, C., \& Tauschek, M. (2015). Between imagined communities and communities of practice: Participation, territory and the making of heritage (Vol. 8). Germany: Universitätsverlag Göttingen.

Adlam, P. (1987). Language and identity: A sociolinguistic survey of the Indonesian speech community in Wellington, New Zealand. (Unpublished MA Thesis). Victoria University of Wellington: New Zealand,

Ahmad, R. (2007). Shifting Dunes: Changing meanings of Urdu in India. (Unpublished $\mathrm{PhD}$ thesis). University of Michigan: The USA,

Al-Sahafi, M. (2015). The role of Arab fathers in heritage language maintenance in New Zealand. International Journal of English Linguistics, 5(1), 73-83. doi:10.5539/ijel.v5n1p73

Al-Sahafi, M., \& Barkhuizen, G. (2006). Language use in an immigrant context: The case of Arabic in Auckland. New Zealand Studies in Applied Linguistics, 12(1), 51-69.

Alatis, J. E., \& Tan, A.-H. (1999). Language in our time: Bilingual education and official English, Ebonics and Standard English, immigration and the Unz Initiative. Georgetown University Round Table on Languages and Linguistics, $1-431$.

Albirini, A. (2016). Modern Arabic sociolinguistics: Diglossia, variation, codeswitching, attitudes and identity. New York: Routledge.

Albury, N. J. (2016). Defining Māori language revitalisation: A project in folk linguistics. Journal of Sociolinguistics, 20(3), 287-311. doi:10.1111/josl.12183

Ammon, U. (2010). World languages: Trends and futures. In N. Coupland (Ed.), The handbook of language and globalisation (pp. 101-122). UK: Blackwell Publishing Ltd.

Anderson, B. (1991). Imagined communities: Reflections on the origin and spread of nationalism. New York: Verso.

Anderson, K. (2008). Justifying race talk: Indexicality and the social construction of race and linguistic value. Journal of Linguistic Anthropology, 18(1), 108-129. doi:10.1111/j.1548-1395.2008.00017.x

Angouri, J. (2018). Culture, discourse, and the workplace. London: Routledge.

Atkinson, D. (2016). The burden of white supremacy: Containing Asian migration in the British Empire and the United States. Chapel Hill: The University of North Carolina Press.

Atkinson, P. (2004). Performance, culture and the sociology of education. International Studies in Sociology of Education, 14(2), 147-168. doi:10.1080/09620210400200123

Ayeomoni, M. (2011). Language, food and culture: Implications for language development and expansion in Nigeria. Advances in Bioresearch, 2(2), 50-55. 
Bailey, B. (2007). Multilingual forms of talk and identity work. In P. Auer \& L. Wei (Eds.), Handbook of multilingualism and multilingual communication (pp. 341369). Berlin: Mouton de Gruyter.

Baker, C. (2006). Foundations of bilingual education and bilingualism (4th ed.). Sydney: Multilingual Matters.

Bakhtin, M. (1994). Double-voiced discourse in Dostoevsky. In P. Morris (Ed.), The Bakhtin reader: Selected writings (pp. 102-111). London: Edward Arnold.

Balirano, G., \& Guzzo, S. (Eds.). (2019). Food across cultures: Linguistic insights in transcultural tastes. Cham: Palgrave Macmillan.

Bandyopadhyay, S. (Ed.) (2010). India in New Zealand: Local identities, global relations. Dunedin: Otago University Press.

Baralt, M. (2012). Coding qualitative data. In A. Mackey \& S. Gass (Eds.), Research methods in second language acquisition: A practical guide (1st ed., pp. 222244). Chichester: Wiley-Blackwell.

Barkhuizen, G. (2003). "They'll be Kiwis one day”: Afrikaans-speaking parents' reflections on their children's shifting language behavior. Paper presented at the Paper presented at the 13th Australia TESOL conference, Sydney, Australia.

Barkhuizen, G. (2006). Immigrant parents' perceptions of their children's language practices: Afrikaans speakers living in New Zealand. Language Awareness, 15(2), 63-79. doi:10.1080/09658410608668851

Barkhuizen, G. (2015). Learning English with a home tutor: meeting the needs of migrant learners. In D. Nunan \& J. C. Richards (Eds.), Language learning beyond the classroom (pp. 282-291). New York: Routledge.

Barkhuizen, G., \& De Klerk, V. (2006). Imagined identities: Preimmigrants' narratives on language and identity. International Journal of Bilingualism, 10(3), 277-299.

Bauman, Z. (1992). Intimations of postmodernity.

Baumgarten, N., \& House, J. (2010). I think and I don't know in English as lingua franca and native English discourse. Journal of Pragmatics, 42(5), 1184-1200.

Bazeley, P., \& Jackson, K. (2013). Qualitative data analysis with NVivo (2nd ed.). London: Sage.

Bell, A. (1984). Language style as audience design. Language in Society, 13(2), 145204.

Bell, A., Harlow, R., \& Starks, D. (Eds.). (2005). Languages of New Zealand. Wellington: Victoria University Press.

Belz, J. (2002). The myth of the deficient communicator. Language Teaching Research, 6(1), 59-82. doi:10.1191/13621688021r097oa

Benhabib, S. (1996). The Democratic Movement and the Problem of Difference. In S. Benhabib (Ed.), Democracy and difference: Contesting the boundaries of the Political (pp. 3-18). Princeton, NJ: Princeton University Press.

Benton, N. (1989). Education, language decline and language revitalisation: The case of Māori in New Zealand. Language and Education, 3(2), 65-82. doi:10.1080/09500788909541252

Beres, A. (2015). An overview of translanguaging: 20 years of 'giving voice to those who do not speak'. Translation and translanguaging in multilingual contexts, 1(1), 103-118. doi:10.1075/ttmc.1.1.05ber 
Berg, B. (2007). Qualitative Research Methods for the Social Sciences (6th ed.). Boston: Allyn \& Bacon.

Berg, B. (2009). Qualitative Research Methods for the Social Sciences (7th ed.). Boston: Pearson.

Best, S. (2013). Understanding and doing successful research: Data collection and analysis for the social sciences. London: Routledge.

Beswick, J. (2007). Regional nationalism in Spain: Language use and ethnic identity in Galicia. Clevedon: Multilingual matters.

Bissoonauth, A. (2011). Language shift and maintenance in multilingual Mauritius: The case of Indian ancestral languages. Journal of Multilingual and Multicultural Development, 32(5), 421-434. doi:10.1080/01434632.2011.586463

Blackledge, A., \& Creese, A. (2017). A linguistic ethnography of identity: Adopting a heteroglossic frame. In S. Preece (Ed.), The Routledge Handbook of Language and Identity (pp. 272-288). London: Routledge.

Blackledge, A., \& Pavlenko, A. (2001). Negotiation of identities in multilingual contexts. In. London: The Sage.

Bloch, A., \& Hirsch, S. (2017). "Second generation" refugees and multilingualism: identity, race and language transmission. Ethnic and Racial Studies, 1-19. doi:10.1080/01419870.2016.1252461

Blommaert, J. (2005). Discourse: A critical introduction. Cambridge: Cambridge University Press.

Blommaert, J. (2010). The sociolinguistics of globalization. Cambridge: Cambridge University Press.

Blommaert, J., \& Jie, D. (2010). Ethnographic fieldwork: A beginner's guide. Bristol: Multilingual Matters.

Blommaert, J., \& Rampton, B. (2012). Language and superdiversity. MMG Working Paper 12-09, 1-36.

Blum-Kulka, S., Huck-Taglicht, D., \& Avni, H. (2004). The social and discursive spectrum of peer talk. Discourse Studies, 6(3), 307. doi:10.1177/1461445604044291

Blum-Kulka, S., \& Snow, C. (2004). Introduction: The potential of peer talk. Discourse Studies, 6(3), 291-306. doi:10.1177/1461445604044290

Bogdan, R. C., \& Biklen, S. K. (2007). Research for education: An introduction to theories and methods. Boston, MA: Allen and Bacon.

Bourdieu, P. (1977). The economics of linguistic exchanges. Information (International Social Science Council), 16(6), 645-668.

Bourdieu, P. (1986). The forms of capital. In J. Richardson (Ed.), Handbook of Theory and Research for the Sociology of Education. New York: Greenwood.

Bourdieu, P. (1987). What makes a social class? On the theoretical and practical existence of groups. Berkeley journal of sociology, 32, 1-17.

Bourdieu, P. (1990). The logic of practice. Stanford: Stanford university press.

Bourdieu, P. (1991). Language and symbolic power. Cambridge: Polity Press.

Boyce, M. (1992). Māori language in Porirua: A study of reported proficiency, patterns of use, and attitudes. (Unpublished MA Thesis). Victoria University of Wellington: New Zealand., 
Brann, C., \& Benedict, M. (1989). Lingua minor, franca and nationalis. In U. Ammon (Ed.), Status and function of languages and language varieties (pp. 372-385). Berlin: Walter de Gruyter.

Brass, P. (2005). Language, religion and politics in North India (2nd ed.). Lincoln, NE: IUniverse Inc.

Brinton, D., Kagan, O., \& Bauckus, S. (2008). Heritage language education: A new field emerging. New York: Routledge.

Brown, J. R., \& Carpenter, B. (2018). Heritage Somali and identity in rural Wisconsin. Journal of Language Contact, 11(2), 348-371. doi:10.1163/19552629-01102008

Bucholtz, M. (1999). 'Why Be Normal?': Language and Identity Practices in a Community of Nerd Girls. Language in Society, 28(2), 203-223.

Bucholtz, M., \& Hall, K. (2004). Theorizing identity in language and sexuality research. Language in Society, 33(4), 469-515. doi:10.1017/S0047404504334020

Bucholtz, M., \& Hall, K. (2005). Identity and interaction: A sociocultural linguistic approach. Discourse Studies, 7(4-5), 585-614. doi:10.1177/1461445605054407

Bucholtz, M., \& Hall, K. (2010). Locating identity in language. In C. Llamas \& D. Watt (Eds.), Language and identities (pp. 18-28). Edinburgh: Edinburgh University Press.

Bucholtz, M., \& Hall, K. (2016). Embodied sociolinguistics. In N. Coupland (Ed.), Sociolinguistics: Theoretical Debates (pp. 173-197). Cambridge: Cambridge University Press.

Burke, P. (2009). Identity theory. Oxford: Oxford University Press.

Busch, B. (2012). The linguistic repertoire revisited. Applied Linguistics, 33(5), 503523. doi:10.1093/applin/ams056

Caglar, A. (1997). Hyphenated identities and the limits of 'culture' In T. Modood \& P. Werbner (Eds.), The politics of multiculturalism in Europe: Racism, identity and community (pp. 169-185). New York: Zed Books Ltd.

Cameron, D., Frazer, E., Harvey, P., Rampton, M., \& Richardson, K. (2018). Researching language: Issues of power and method. London: Routledge.

Campbell, R., \& Rosenthal, J. (2000). Heritage languages. In J. Rosenthal (Ed.), Handbook of undergraduate second language education (pp. 165-184). New Jersey Lawrence Erlbaum Associates.

Canagarajah, S. (2008). Language shift and the family: Questions from the Sri Lankan Tamil diaspora. Journal of Sociolinguistics, 12(2), 143-176.

Canagarajah, S. (2013). Reconstructing heritage language: Resolving dilemmas in language maintenance for Sri Lankan Tamil migrants. International Journal of the Sociology of Language, 222(222), 131-155. doi:10.1515/ijsl-2013-0035

Cherciov, M. (2012). Investigating the impact of attitude on first language attrition and second language acquisition from a Dynamic Systems Theory perspective. International Journal of Bilingualism, 17(6), 716-733. doi:10.1177/1367006912454622

Christensen, I. (2003). Proficiency, use and transmission: Māori language revitalisation. New Zealand Studies in Applied Linguistics, 9(1), 41-61.

Christopher, K. (1994). One language, two scripts: The Hindi movement in nineteenth century North India. Oxford: Oxford University Press. 
Coffey, S. (2015). Reframing teachers' language knowledge through metaphor analysis of language portraits. Modern Language Journal, 99(3), 500-514. doi:10.1111/modl.12235

Cogo, A. (2016). Conceptualizing ELF as a translanguaging phenomenon: Covert and overt resources in a transnational workplace. Waseda Working Papers in ELF, 5, 61-77.

Cook, H. M. (2008). Socializing identities through speech style: Learners of Japanese as a foreign language. Bristol: Multilingual Matters.

Copland, F., \& Creese, A. (Eds.). (2015). Linguistic ethnography: Collecting, analysing and presenting data. London: Sage.

Corbin, J., \& Strauss, A. (2008). Basics of qualitative research: Techniques and procedures for developing grounded theory (3rd ed.). Los Angeles: SAGE Publications.

Cote, J. E., \& Levine, C. G. (2014). Identity, formation, agency, and culture: A social psychological synthesis. Mahwah, N.J.: Lawrence Erlbaum Associates.

Council of Europe. (2005). Council of Europe framework convention on the value of cultural heritage for society (Farp Conventions, 199). Retrieved from https://rm.coe.int/1680083746

Coupland, N. (2007). Style: Language variation and identity. Cambridge: Cambridge University Press.

Creese, A. (2008). Linguistic ethnography. In K. King \& N. Hornberger (Eds.), Encyclopedia of language and education (2nd ed., Vol. 10: Research Methods in Language and Education, pp. 229-241). USA: Springer Science+BusinessMedia LLC.

Creese, A. (2010). Linguistic ethnography. In L. Litosseliti (Ed.), Research methods in linguistics (pp. 138-154). London: Continuum.

Creese, A., Blackledge, A., \& Takhi, J. K. (2014). The ideal 'native speaker'teacher: Negotiating authenticity and legitimacy in the language classroom. The Modern Language Journal, 98(4), 937-951.

Creese, A., \& Copland, F. (2017). Linguistic ethnography. In K. King, Y.-J. Lai, \& S. May (Eds.), Research methods in language and education (3rd ed., pp. 339-351). Cham: Springer.

Crezee, I. (2012). Language shift and host society attitudes: Dutch migrants who arrived in New Zealand between 1950 and 1965. International Journal of Bilingualism, 16(4), 528-540. doi:10.1177/1367006911429523

Cummins, J. (2000). Forward. In R. Gonzales \& I. Melis (Eds.), Language ideologies: Education and the social implications of official language (pp. ix-xxi). New Jersey: Lawrence Erlbaum.

Darvin, R., \& Norton, B. (2015). Identity and a model of investment in applied linguistics. Annual Review of Applied Linguistics, 35, 36-56. doi:10.1017/S0267190514000191

Davies, B. (2005). Communities of practice: Legitimacy not choice. Journal of Sociolinguistics, 9(4), 557-581. doi:10.1111/j.1360-6441.2005.00306.x

Davies, B., \& Harré, R. (1990). Positioning: The discursive production of selves. Journal for the Theory of Social Behaviour, 20(1), 43-63. doi:10.1111/j.14685914.1990.tb00174.x 
Davis, K., \& Starks, D. (2005). Four factors for Cook Island Māori language maintenance. In A. Bell, R. Harlow, \& D. Starks (Eds.), Languages of New Zealand (pp. 298-321). Wellington: Victoria University Press.

Day, E. M. (2002). Identity and the young English language learner. Clevedon: Multilingual Matters.

De Bres, J. (2011). Promoting the Māori language to non-Māori: Evaluating the New Zealand government's approach. Language Policy, 10(4), 361-376. doi:10.1007/s10993-011-9214-7

De Bres, J. (2015). The hierarchy of minority languages in New Zealand. Journal of Multilingual and Multicultural Development, 36(7), 677-693. doi:10.1080/01434632.2015.1009465

De Bres, J. (2017). Singing is the first thing that came into my head: Emotions and language maintenance among Filipino migrants in New Zealand. In M. Marra \& P. Warren (Eds.), Linguist at work: Festschrift for Janet Holmes (pp. 137-158). Wellington: Victuria University Press.

De Bres, J. (2019). Negotiating meaning through drawing in research on multilingualism. Paper presented at the Paper presented at the 6th Interaction and Meaning (iMean6), Wellington, New Zealand.

De Cillia, R., Reisigl, M., \& Wodak, R. (1999). The discursive construction of national identities. Discourse \& Society, 10(2), 149-173.

De Fina, A. (2015). Narrative and identities. In A. De Fina \& A. Georgakopoulou (Eds.), The handbook of narrative analysis (pp. 351-368). Malden, MA: Wiley Blackwell.

De Fina, A., \& Perrino, S. (2013). Transnational identities. Applied Linguistics, 34(5), 509-515. doi:10.1093/applin/amt024

De Fina, A., Schiffrin, D., \& Bamberg, M. (Eds.). (2006). Discourse and identity. Cambridge: Cambridge University Press.

De Fina, A., \& Tseng, A. (2017). Narrative in the study of immigration. In S. Canagarajah (Ed.), The Routledge handbook of migration and language (pp. 381-396). New York: Routledge.

De Swaan, A. (2010). Language systems. In N. Coupland (Ed.), The handbook of language and globalization (pp. 56-76). UK: Blackwell Publishing Ltd.

Deschambault, R. (2017). Actively managed products: Think-aloud data and methods in applied linguistics research. Applied Linguistics Review, O(0). doi:10.1515/applirev-2017-0028

Desmond, C. (2017). Effect of linguistic integration in dual language on achievement and investment. (Unpublished $\mathrm{PhD}$ thesis). California State University San Marcos: The USA,

Dorian, N. (1983). Language loss and maintenance in language contact situations. In L. Freed (Ed.), The loss of language skills (pp. 44-59). New York: Rowley.

Drager, K. (2015). Linguistic variation, identity construction and cognition. Berlin: Language Sceince Press.

Draper, J., \& Hicks, J. (2000). Where we've been; what we've learned. In J. Webb \& B. Miller (Eds.), Teaching heritage language learners: Voices from the classroom (pp. 15-35). New York: ACTFL. 
Dressler, R. (2014). Exploring Linguistic Identity in Young Multilingual Learners. TESL Canada Journal, 32(1), 42-52. doi:10.18806/tesl.v32i1.1198

Dua, H. (2006). Hindustani. In K. Brown, L. Bauer, M. S. Berns, J. E. Miller, \& G. Hirst (Eds.), Encyclopedia of language and linguistics (2nd ed., Vol. 1, pp. 309312). Amsterdam: Elsevier.

Dweik, B. (1985). Future of the Arabic language in America: A sociolinguistic Study. Palestine: Sharbain's Bookshop.

Dweik, B. (2000). Linguistic and cultural maintenance among the Chechens of Jordan. Language, Culture and Curriculum, 13(2), 184-195. doi:10.1080/07908310008666598

Dweik, B., Nofal, M., \& Al-Obaidi, M. (2019). An ethnic language and culture without a safe enclave: A socio-cultural perspective. International Journal of ArabicEnglish Studies, 19(1), 103-123. doi:10.33806/ijaes2000.19.1.6

Eastman, C. (1984). Language, ethnic identity and change. In J. Edwards (Ed.), Linguistic minorities, policies and pluralism (pp. 259-276). London: Academic Press.

Eckert, P. (2000). Linguistic variation as social practice: The linguistic construction of identity in Belten High. Oxford: Blackwell.

Eckert, P. (2008). Variation and the indexical field. Journal of Sociolinguistics, 12(4), 453-476. doi:10.1111/j.1467-9841.2008.00374.x

Eckert, P., \& McConnell-Ginet, S. (1992). Think practically and look locally: Language and gender as community- based practice. Annual Review of Anthropology, 21, 461-490.

Eckert, P., \& McConnell-Ginet, S. (2013). Language and gender (2nd ed.). Cambridge; New York: Cambridge University Press.

Edwards, J. (1994). Multilingualism. London: Routledge.

Edwards, J. (2009). Language and identity: An introduction. Cambridge: Cambridge University Press.

Edwards, J. (2010). Minority languages and group identity: Cases and categories. Amsterdam: John Benjamins.

Eliasson, S. (2015). The birth of language ecology: Interdisciplinary influences in Einar Haugen's "The ecology of language". Language Sciences, 50, 78-92. doi:10.1016/j.langsci.2015.03.007

Ellis, E. M., Sims, M., \& Knox, V. (2018). The challenge of isolation in immigrant family language maintenance in regional Australia. Journal of Home Language Research, 3, 17-35.

Fereday, J., \& Muir-Cochrane, E. (2006). Demonstrating rigor using thematic analysis: A hybrid approach of inductive and deductive coding and theme development. International journal of qualitative methods, 5(1), 80-92.

Fishman, J. (1964). Language maintenance and language shift as a field of inquiry: A definition of the field and suggestions for its further development. LinguisticsAn Interdisciplinary Journal of the Language Sciences(9), 32-70.

Fishman, J. (1965). Who speaks what language to whom and when? La Linguistique, $1(2), 67-88$. 
Fishman, J. (1966). Language loyalty in the United States: The maintenance and preparation of non-English mother tongues by American ethnic and religious groups. The Hauge: Mouton \& CO.

Fishman, J. (1985). Language maintenance and ethnicity. In J. Fishman, M. Gertner, E. Lowy, \& W. Milán (Eds.), The rise and fall of the ethnic revival: Perspectives on language and ethnicity (pp. 57-76). Berlin: De Gruyter Mouton.

Fishman, J. (1989). Language and ethnicity in minority sociolinguistic perspective. England: Multilingual Matters LTD.

Fishman, J. (1991a). Reversing language shift: Theoretical and empirical foundations of assistance to threatened languages. Clevedon: Multilingual Matters.

Fishman, J. (1991b). Yiddish: Turning to life. Philadelphia: John Benjamins Publishing. Fishman, J. (2013). Language maintenance and language shift as a field of inquiry: A definition of the field and suggestions for its further development. Linguistics, 51(Jubilee), 9-10. doi:10.1515/ling-2013-0038

Fishman, J. (2014 [2001]). Three hundred-plus years of heritage language education in the United States. In T. G. Wiley, J. Kreeft Peyton, D. Christian, S. C. K. Moore, \& N. Liu (Eds.), Handbook of heritage, community, and native American languages in the United States: Research, policy, and educational practice (pp. 36-44). London: Routledge.

Fletcher, J. (2014). Social communities in a knowledge enabling organizational context: Interaction and relational engagement in a community of practice and a microcommunity of knowledge. Discourse \& Communication, 8(4), 351-369. doi:10.1177/1750481314537577

Fogle, L. (2012). Second language socialization and learner agency: Adoptive family talk. Clevedon, UK: Multilingual Matters.

Fogle, L. (2013). Parental ethnotheories and family language policy in transnational adoptive families. Language Policy, 12(1), 83-102. doi:10.1007/s10993-0129261-8

Fogle, L., \& King, K. (2013). Child agency and language policy in transnational families. Issues in Applied Linguistics, 19(0), 1-25.

Folmer, J. (1992). Dutch immigrants in New Zealand: A case study of language shift and language loss. Australian Review of Applied Linguistics, 15(2), 1-18.

Foucault, M. (1977). Discipline and punish: The birth of the prison. New York: Pantheon Books.

Friesen, W. (2008). The evolution of 'Indian' identity and transnationalism in New Zealand. Australian Geographer, 39(1), 45-61. doi:10.1080/00049180701877428

Fuchs, M., Linkenbach, A., \& Malik, A. (2010). What does it mean to be Indian? A view from Christchurch. In S. Bandyopadhyay (Ed.), India in New Zealand: Local identities, global relations (pp. 81-106). Dunedin: Otago University Press.

Gabaccia, D. (2000). We are what we eat: Ethnic food and the making of Americans. Cambridge, MA: Harvard University Press.

Gal, S. (1979). Language shift: Social determinants of linguistic change in bilingual Austria. New York: Academic Press.

Gambhir, S., \& Gambhir, V. (2014). The journey of Hindi in the United States. In T. G. Wiley, J. Kreeft Peyton, D. Christian, S. C. K. Moore, \& N. Liu (Eds.), 
Handbook of heritage, community, and native American languages in the United States: Research, policy, and educational practice (pp. 202-212). New York: Routledge.

Gambhir, V. (1995). Developing reading proficiency in modern South Asian languages. In V. Gambhir (Ed.), The Teaching and Acquisition of South Asian Languages (pp. 108-135). Philadelphia: University of Pennsylvania Press.

Gao, F. (2012). Imagined community, identity, and Chinese language teaching in Hong Kong. Journal of Asian Pacific Communication, 22(1), 140-154.

Garcia, M. (2003). Recent research on language maintenance. Annual Review of Applied Linguistics, 23, 22-43.

García, O. (2005). Positioning heritage languages in the United States. The Modern Language Journal, 89(4), 601-605.

García, O. (2009). Bilingual Education in the 21st century: A Global Perspective. Malden, MA: Blackwell.

García, O., \& Li, W. (2014). Translanguaging: Language, bilingualism and education. New York: Palgrave Macmillan.

Gee, J. P. (1996). Social linguistics and literacies: Ideology in discourses (2nd ed.). London: Taylor \& Francis.

Gerhardt, C. (2013). Introduction. In C. Gerhardt, M. Frobenius, \& S. Ley (Eds.), Culinary linguistics: The chef's special (pp. 3-49). Amsterdam: John Benjamins Publishing Company.

Gerwinski, J., \& Linz, E. (2018). Methodik II: Beobachterparadoxon - die Aufnahmesituation im Gespräch. In J. Gerwinski, S. Habscheid, \& E. Linz (Eds.), Theater im Gespräch: Sprachliche Publikumspraktiken in der Theaterpause (pp. 105-163). Berlin: De Gruyter.

Gharibi, K. (2016). Incomplete acquisition, attrition and maintenance of heritage speakers' family Language: Iranians in New Zealand. (Unpublished PhD thesis). Victoria University of Wellington: New Zealand,

Gharibi, K., \& Seals, C. (2019). Family language policy towards heritage language literacy acquisition and maintenance: Iranians in New Zealand. In S. H. Mirvahedi (Ed.), The sociolinguistics of Iran's languages at home and abroad: The case of Persian, Azerbaijani, and Kurdish (pp. 109-139). Cham: Palgrave Macmillan.

Gibbs, R. (2003). Embodied Experience and Linguistic Meaning. Brain and Language, 84(1), 1-15. doi:10.1016/S0093-934X(02)00517-5

Gilbertson, A. (2010). Choosing Indian and Kiwi identities: The ethnic options of New Zealand-born Gujaratis. In S. Bandyopadhyay (Ed.), India in New Zealand: Local identities, global relations (pp. 123-140). Dunedin: Otago University Press.

Giles, H. (1973). Accent mobility: A model and some data. Anthropological Linguistics, 15(2), 87-105.

Giles, H., Bourhis, R., \& Taylor, D. (1977). Towards a theory of language in ethnic group relations. In H. Giles (Ed.), Language, ethnicity and intergroup relations (pp. 307-348). London: Academic Press.

Giles, H., \& Coupland, N. (1991). Language attitudes: Discursive, contextual, and gerontological considerations. Paper presented at the Bilingualism, 
multiculturalism, and second language learning: The McGill Conference in honour of Wallace E. Lambert.

Glesne, C. (2011). Becoming qualitative researchers: An introduction (4th ed. ed.). Boston: Pearson.

Goodz, N. (1994). Interactions between parents and children in bilingual families. In F. Genesee (Ed.), Educating second language children: The whole child, the whole curriculum, the whole community (pp. 61-81). Cambrige: Cambridge University Press.

Gordon, C. (2009). Making meanings, creating family: Intertextuality and framing in family interaction. New York: Oxford University Press.

Gordon, C. (2012). Beyond the observer's paradox: The audio-recorder as a resource for the display of identity. Qualitative research, 13(3), 299-317. doi:10.1177/1468794112442771

Gorter, D., Marten, H., Jansma, L., \& Ytsma, J. (Eds.). (1990). Fourth international conference on minority languages. Volume II: Western and Eastern European papers (Vol. 2). Clevedon: Multilingual matters.

Groff, C. (2018). Language policy and language ideology: ecological perspectives on language and education in the Himalayan foothills. Anthropology \& Education Quarterly, 49(1), 3-20. doi:10.1111/aeq.12235

Guardado, M. (2008). Language socialization in Canadian Hispanic communities: Ideologies and practices. (Unpublished $\mathrm{PhD}$ thesis). University of British Columbia, Vancouver, Canada,

Guardado, M. (2013). The metapragmatic regimentation of heritage language use in Hispanic Canadian caregiver-child interactions. International Multilingual Research Journal, 7(3), 230-247.

Guardado, M. (2014). The discourses of heritage language development: Engaging ideologies in Canadian Hispanic communities. Heritage Language Journal, $11(1), 1-28$.

Guardado, M. (2018a). Discourse, ideology and heritage language socialization: Micro and macro perspectives. Boston: De Gruyter.

Guardado, M. (2018b). Spanish as a minority/heritage language in Canada and the UK. In K. Potowski (Ed.), The Routledge Handbook of Spanish as a Heritage Language (pp. 537-554). New York: Routledge.

Gumperz, J. (1982). Discourse strategies. Cambridge: Cambridge University Press.

Guo, Z. (2014). Young children as intercultural mediators. Bristol: Multilingual Matters.

Guzzo, S., \& Gallo, A. (2019). Diasporic identities in social practices: Language and food in the Loughborough

Italian community. In G. Balirano \& S. Guzzo (Eds.), Food across cultures: Linguistic insights in transcultural tastes (pp. 71-98). Cham: Palgrave Macmillan.

Häberl, C. (2006). The Neo-Mandaic dialect of Khorramshahr. (Unpublished Ph.D Thesis). Harvard University: The USA.,

Hallett, D., Chandler, M. J., \& Lalonde, C. E. (2007). Aboriginal language knowledge and youth suicide. Cognitive development, 22(3), 392-399. 
Han, Y., \& Ellis, R. (1998). Implicit knowledge, explicit knowledge and general language proficiency. Language Teaching Research, 2(1), 1-23. doi:10.1177/136216889800200102

Harris, R., \& Rampton, B. (2003). Language, ethnicity and race reader. London: Routledge.

Hatoss, A. (2012). Where are you from? Identity construction and experiences of 'othering'in the narratives of Sudanese refugee-background Australians. Discourse \& Society, 23(1), 47-68.

Hatoss, A. (2013). Displacement, language maintenance and identity : Sudanese refugees in Australia. Amsterdam / Philadelphia: John Benjamins Publishing Company.

Haugen, E. (1972). The ecology of language. In A. Dil (Ed.), The ecology of language: Essays by Einar Haugen (pp. 325-339). Califirnia: Stanford University Press.

Hay, J. (1994). Jocular abuse patterns in mixed-group interaction. Wellington Working Papers in Linguistics, 26-55.

Hazen, K. (2000). The role of researcher identity in conducting sociolinguistic research: A reflective case study. Southern Journal of Linguistics, 24, 103-120.

Hazenberg, E. (2017). Liminality as a lens on social meaning: A cross-variable study of gender in New Zealand English. (Unpublished $\mathrm{PhD}$ thesis). Victoria University of Wellington: New Zealand,

Heller, M. (2008). Doing ethnography. In L. Wei \& M. Moyer (Eds.), The Blackwell guide to research methods in bilingualism and multilingualism (pp. 249-262). Malden, MA: Blackwell.

Heller, M., Pietikäinen, S., \& Pujolar, J. (2018). Critical sociolinguistic research methods: Studying language issues that matter. London: Routledge.

Hennink, M., Hutter, I., \& Bailey, A. (2020). Qualitative research methods. Los Angeles: SAGE Publications Limited.

Holmes, J. (1993). Immigrant women and language maintenance in Australia and New Zealand. International journal of Applied Linguistics, 3(2), 159-179.

Holmes, J. (1997). Keeping tabs on language shift in New Zealand: Some methodological considerations. Journal of Multilingual and Multicultural Development, 18(1), 17-39. doi:10.1080/01434639708666300

Holmes, J., \& Marra, M. (2002). Having a laugh at work: How humour contributes to workplace culture. Journal of Pragmatics, 34(12), 1683-1710. doi:10.1016/S0378-2166(02)00032-2

Holmes, J., Marra, M., \& Schnurr, S. (2008). Impoliteness and ethnicity: Māori and Pākehā discourse in New Zealand workplaces. Journal of Politeness Research: Language, Behaviour, Culture, 4(2), 193-219.

Holmes, J., Marra, M., \& Vine, B. (2011). Leadership, discourse, and ethnicity. New York: Oxford University Press.

Holmes, J., \& Meyerhoff, M. (1999). The community of practice: Theories and methodologies in language and gender research. Language in Society, 28(2), 173-183.

Holmes, J., Roberts, M., Verivaki, M., \& 'Aipolo, A. (1993). Language maintenance and shift in three New Zealand speech communities. Applied Linguistics, 14(1), $1-24$. 
Holton, J. (2007). The coding process and its challenges. In A. Bryant \& K. Charmaz (Eds.), The Sage handbook of grounded theory (pp. 265-289). Los Angelos: Sage Publications.

Hornberger, N., \& Wang, S. (2008). Who are our heritage language learners? Identity and biliteracy in heritage language education in the United States. In D. Brinton, O. Kagan, \& S. Bauckus (Eds.), Heritage language acquisition: A new field emerging (pp. 3-35). New York: Routledge.

Howie, D., \& Peters, M. (1996). Positioning theory: Vygotsky, Wittgenstein and social constructionist

psychology. Journal for the Theory of Social Behaviour, 26(1), 51-64.

Howitt, D., \& Cramer, D. (2007). Introduction to research methods in psychology: Pearson Education.

Hua, Z. (2014). Exploring intercultural communication language in action. In. New York: Routledge.

Hua, Z. (2017). New orientations to identities in mobility. In S. Canagarajah (Ed.), The Routledge handbook of migration and language (pp. 117-132). New York: Routledge.

Hussain, Y. (2019). 'I know my roots are Indian but my thinking is Kiwi': hybridisation, identity and 'Indians' in New Zealand. South Asian Diaspora, 11(1), 1-15. doi:10.1080/19438192.2018.1464697

Hyland, K. (2017). Metadiscourse: What is it and where is it going? Journal of Pragmatics, 113, 16-29.

Hyland, K. (2019). Metadiscourse: Exploring interaction in writing (2nd ed.). London: Bloomsbury Publishing.

Hyland, K., \& Tse, P. (2004). Metadiscourse in academic writing: A reappraisal. Applied Linguistics, 25(2), 156-177. doi:10.1093/applin/25.2.156

Jamieson, P. (1980). The pattern of urban language loss. The Australian and New Zealand Journal of Sociology, 16(2), 102-109.

Jaworski, A. (1993). The power of silence social and pragmatic perspectives. In. Newbury Park, California: Sage.

Jaworski, A. (1998). Talk and silence in the interrogation. Language and Literature: Journal of the Poetics and Linguistics Association, 7(2), 99-122. doi:10.1177/096394709800700201

Jensen, K. K. (2014). What can and cannot be willed: How politicians talk about national identity and immigrants. Nations and Nationalism, 20(3), 563-583.

Johnson, H., \& Moloughney, B. (2006). Asia in the making of New Zealand. Auckland: Auckland University Press.

Kachru, Y. (2006). Hindi. Amsterdam: John Benjamins.

Kachru, Y. (2009). Hindi-Urdu. In B. Comrie (Ed.), The world's major languages (2nd ed., pp. 398-416). New York: Routledge.

Kalaja, P., Dufva, H., Alanen, R., \& Barkhuizen, G. (2013). Experimenting with visual narratives. Narrative research in applied linguistics, 105-131.

Kandler, A., Unger, R., \& Steele, J. (2010). Language shift, bilingualism and the future of Britain's Celtic languages. Philosophical Transactions: Biological Sciences, 365(1559), 3855-3864. 
Kelleher, A. (2010). Who is a heritage language learner (Heritage Brief), 1-3. Retrieved from http://www.cal.org/heritage/pdfs/briefs/What-is-a-Heritage-Language.pdf

Kheirkhah, M., \& Cekaite, A. (2015). Language maintenance in a multilingual family: Informal heritage language lessons in parent-child interactions. Multilingua, 34(3), 319-346. doi:10.1515/multi-2014-1020

Khubchandani, L. (1989). Diglossia and functional heterogeniety. In U. Ammon (Ed.), Status and function of languages and language varieties (pp. 592-607). Berlin: Walter De Gruyter.

Kiesling, S. (2004). Dude. American Speech, 79(3), 281-305. doi:10.1215/0003128379-3-281

Kim, J. (2019). The role of minority language-speaking families, community and the majority society in the intergenerational language transmission of the Korean language in New Zealand. (Unpublished PhD thesis). University of Canterbury: New Zealand,

Kim, J., \& Starks, D. (2005). Language diaries: A case study of language use in the New Zealand Korean community. In A. Bell, R. Harlow, \& D. Starks (Eds.), Languages of New Zealand (pp. 343-369). Wellington: Victoria University Press.

Kim, S., \& Starks, D. (2010). The role of fathers in language maintenance and language attrition: The case of Korean-English late bilinguals in New Zealand. International Journal of Bilingual Education and Bilingualism, 13(3), 285-301. doi:10.1080/13670050903193958

King, B. (2011). Linguistic negotiations of sexual agency in sexuality education. (Unpublished PhD thesis). Victoria University of Wellington: New Zealand,

King, B. (2019). Communities of practice in language research: A critical introduction. Abingdon: Routledge.

King, K. (2013). A tale of three sisters: Language ideologies, identities, and negotiations in a bilingual, transnational family. International Multilingual Research Journal, 7(1), 49-65. doi:10.1080/19313152.2013.746800

King, K. (2016). Language policy, multilingual encounters, and transnational families. Journal of Multilingual and Multicultural Development, 37(7), 726-733. doi:10.1080/01434632.2015.1127927

King, K., \& Fogle, L. (2017). Family language policy. In T. McCarty \& S. May (Eds.), Language Policy and Political Issues in Education (3rd ed., pp. 315-327). Cham: Springer International Publishing.

King, K., Fogle, L., \& Logan-Terry, A. (2008). Family language policy. Language and Linguistics Compass, 2(5), 907-922. doi:10.1111/j.1749-818X.2008.00076.X

King, K., \& Logan-Terry, A. (2008). Additive bilingualism through family language policy: Ideologies, strategies and interactional outcomes. Calidoscópio, 6(1), 519.

Kloss, H. (1966). German-American language maintenance efforts. In J. Fishman (Ed.), Language loyalty in the United States: The maintenance and preparation of nonEnglish mother tongues by American ethnic and religious groups (pp. 206-252). The Hauge: Mouton \& CO.

Kloss, H. (1967). Abstand languages and Ausbau languages. Anthropological Linguistics, 9(7), 29-41. 
Kress, G. (2010). Multimodality: A social semiotic approach to contemporary communication. London: Routledge.

Kroskrity, P. (1999a). Identity. Journal of Linguistic Anthropology, 9(1/2), 111-114.

Kroskrity, P. (1999b). Language ideologies, language shift, and the imagination of a Western Mono Community: The recontextualization of a Coyote story. In J. Verschueren (Ed.), Language and ideology: Selected papers from the 6th International Pragmatics Conference (Vol. 1, pp. 270-289). Antwerp, Belgium: International Pragmatics Association.

Krumm, H.-J. (2004). Heterogeneity: Multilingualism and democracy. Utbildning \& Demokrati, 13(3), 61-77.

Krumm, H.-J. (2013). Multilingualism and identity: What linguistic biographies of migrants can tell us. In P. Siemund, I. Gogolin, \& M. E. Schulz (Eds.), Multilingualism and language diversity in urban areas acquisition, identities, space, education (pp. 165-176). Philadelphia: John Benjamins Publishing Company.

Krumm, H.-J., \& Jenkins, E.-M. (2001). Kinder und ihre Sprachen - lebendige Mehrsprachigkeit: Sprachenportraits [Children and their language - living multililingualism: Language portraits]. Vienna: Wiener VerlagsWerkstatt für Interkulturelles Lernen und Deutsch als Fremdsprache.

Kühl, K., \& Peterson, E. (2018). The remains of the Danes: The final stages of language shift in Sanpete County, Utah. Journal of Language Contact, 11(2), 208-232.

Kuiper, K. (2005). Invisible immigrants, inaudible language: Nederlands en Nederladers in Nieuw Zeeland. In A. Bell, R. Harlow, \& D. Starks (Eds.), Languages of New Zealand (pp. 322-342). Wellington: Victoria University Press.

Kulavuz-Onal, D. (2018). Fiona Copland and Angela Creese (with Frances Rock and Sara Shaw). Linguistic Ethnography: Collecting, Analyzing, and Presenting Data. London: Sage Publications. 2015. 280 pp. Pb (9781446257388) US\$48.00. In (Vol. 22, pp. 118-122).

Kumar, D. Z. (2018). Identity changes in the Slovenian and Friulian linguistic communities in the Province of Udine, Friuli-Venezia Giulia, Italy. European Countryside, 10(1), 141-157.

Labov, W. (1966). The social stratification of English in New York City. USA: Center for Applied Linguistics.

Labov, W. (1972). Sociolinguistic patterns. Pennsylvania: University of Pennsylvania Press.

Lakoff, R. (2006). Identity à la carte: You are what you eat. In A. De Fina, D. Schiffrin, \& M. Bamberg (Eds.), Discourse and Identity (pp. 142-165). Cambridge: Cambridge University Press.

Lal, B. (2004). Girmitiyas: The origins of the Fiji Indians. Lautoka: Fiji Institute of Applied Sciences.

Lamont, M., \& Lareau, A. (1988). Cultural capital: Allusions, gaps and glissandos in recent theoretical developments. Sociological theory, 6(2), 153-168. doi:10.2307/202113

Lange, C. (2012). The syntax of spoken Indian English: John Benjamins Publishing Company. 
Langenhove, L. v., \& Harré, R. (1999). Introducing positioning theory. In R. Harré, L. v. Langenhove, \& L. Berman (Eds.), Positioning theory : Moral contexts of intentional action (pp. 15-31). Malden: Blackwell.

Lanza, E. (1992). Can bilingual two-year-olds code-switch? Journal of Child Language, 19(3), 633-658.

Lanza, E. (2004). Language mixing in infant bilingualism : A sociolinguistic perspective. Oxford: Oxford University Press.

Lasagabaster, D. (2008). Basque diaspora in the USA and language maintenance. Journal of Multilingual and Multicultural Development, 29(1), 66-90. doi:10.2167/jmmd567.0

Lave, J., \& Wenger, E. (1991). Situated learning: Legitimate peripheral participation. Cambridge: Cambridge University Press.

Le Page, R. B., \& Tabouret-Keller, A. (1985). Acts of identity: Creole-based approaches to language and ethnicity. Cambridge: Cambridge University Press.

Leckie, J. (1995). South Asians: Old and new migrations. In S. Greif (Ed.), Immigration and national identity in New Zealand: One people, two Peoples, many peoples? (pp. 133-160). Palmerston North: Dunmore Press.

Lee, S. (2013). Spanish language maintenance and shift among the Chilean community in Auckland. (Unpublished MA thesis). Auckland University of Technology: New Zealand,

Leeman, J., Rabin, L., \& Román-Mendoza, E. (2011). Identity and activism in heritage language education. Modern Language Journal, 95(4), 481-495. doi:10.1111/j.1540-4781.2011.01237.x

Levon, E. (2014). Ethnography and recording interaction. In R. Podesva \& D. Sharma (Eds.), Research methods in linguistics (pp. 195-215). Cambridge: Cambridge University Press.

Lewis, P., \& Simons, G. (2010). Assessing endangerment: Expanding Fishman's GIDS. Revue roumaine de linguistique, 55(2), 103-120.

Lewis, P., Simons, G., \& Fennig, C. (Eds.). (2017). Ethnologue: Languages of the world, Eighteenth edition. Dallas, Texas: SIL International. Online version: http://www.ethnologue.com.

Li, G. (2006). The role of parents in heritage language maintenance and development: Case studies of Chinese immigrant children's home practices. In K. KondoBrown (Ed.), Heritage language development: Focus on East Asian immigrants (pp. 15-31). Philadelphia, PA: John Benjamins Publishing.

Lichtman, M. (2013). Qualitative research for the social sciences. Los Angeles: The Sage.

Lo-Philip, S. W.-Y. (2010). Towards a theoretical framework of heritage language literacy and identity processes. Linguistics and Education, 21(4), 282-297.

Lo, A. (2009). Lessons about respect and affect in a Korean heritage language school. Linguistics and Education, 20(3), 217-234. doi:10.1016/j.linged.2009.07.002

Lo Bianco, J. (2010). Language policy and planning. In N. Hornberger \& S. McKay (Eds.), Sociolinguistics and language education (pp. 143-174). New York: Multilingual Matters. 
Luykx, A. (2005). Children as socializing agents: Family language policy in situations of language shift. Paper presented at the The 4th International Symposium on Bilingualism.

Lynch, J. (1998). Pacific languages: An introduction. USA: University of Hawaii Press. Lyutykh, E. (2011). Russian language schools: Exploring heritage literacy beliefs and practices in families, instruction, and communities. (Unpublished $\mathrm{PhD}$ thesis). Northern Illinois University: The USA,

Macalister, J., \& Mirvahedi, S. H. (2017). Family language policies in a multilingual world: Opportunities, challenges, and consequencies. New York: Routledge.

MacNaughton, G., Davis, K., \& Smith, K. (2009). Exploring "Race-Identities" with young children: Making politics visible. In G. MacNaughton \& K. Davis (Eds.), Race and early childhood education: An international approach to identity, politics, and pedagogy (pp. 31-47). New York: Palgrave Macmillan.

Marra, M., \& Lazzaro-Salazar, M. (2018). Ethnographic methods in pragmatics. In A. Jucker, K. Schneider, \& W. Bublitz (Eds.), Methods in Pragmatics (pp. 343366). Berlin/: De Gruyter Mouton.

Martin-Beltrán, M. (2010). Positioning proficiency: How students and teachers (de)construct language proficiency at school. Linguistics and Education, 21(4), 257-281.

Martin, B. (2012). Coloured language: identity perception of children in bilingual programmes. Language Awareness, 21(1-2), 33-56. doi:10.1080/09658416.2011.639888

Mauthner, M. (1997). Methodological aspects of collecting data from children: Lessons from three research projects. Children \& society, 11(1), 16-28.

May, S. (2004). Rethinking linguistic human rights: Answering questions of identity, essentialism and mobility. In J. Freeland \& D. Patrick (Eds.), Language rights and language survival (pp. 35-53). Manchester, UK: St. Jerome Publishing.

May, S. (2012). Language and minority rights : Ethnicity, nationalism and the politics of language (2nd ed.). New York: Routledge.

May, S., \& Hill, R. (2005). Māori-medium Education: Current issues and challenges. The International Journal of Bilingual Education and Bilingualism, 8(5), 377403.

McKinley, J. (2020). Introduction: Theorizing research methods in the 'golden age' of applied linguistics research. Abingdon: Routledge.

McKinley, J., \& Rose, H. (2020). The Routledge handbook of research methods in applied linguistics. Abingdon: Routledge.

Melo-Pfeifer, S. (2015). Multilingual awareness and heritage language education: Children's multimodal representations of their multilingualism. Language Awareness, 24(3), 197-215. doi:10.1080/09658416.2015.1072208

Melo-Pfeifer, S. (2017). Drawing the plurilingual self: how children portray their plurilingual resources. IRAL - International Review of Applied Linguistics in Language Teaching, 55(1), 41-60. doi:10.1515/iral-2017-0006

Melo-Pfeifer, S., \& Schmidt, A. (2012). Linking "heritage language" education and plurilingual repertoires development: Evidences from drawings of Portuguese pupils in Germany. L1 Educational Studies in Language and Literature, 12, 130. doi:10.17239/L1ESLL-2012.02.11 
Menard-Warwick, J. (2007). 'Because she made beds. Every day'. Social positioning, classroom discourse, and language learning. Applied Linguistics, 29(2), 267289.

Mendoza-Denton, N. C. (1997). Chicana/Mexicana identity and linguistic variation: An ethnographic and sociolinguistic study of gang affiliation in an urban high school. (Unpublished MA Thesis). Stanford University: USA,

Menken, K., \& García, O. (Eds.). (2010). Negotiating language policies in schools: Educators as policymakers. New York and Oxon: Routledge.

Meyerhoff, M. (2013). Place and purpose: Indexicality in ecological perspective. In W. Vandenbussche, E. H. Jahr, \& P. Trudgill (Eds.), Language ecology for the 21 st century: Linguistic conflict and social environments (pp. 267-291). Oslo: Novus Press.

Meyerhoff, M., Schleef, E., \& MacKenzie, L. (2015). Doing sociolinguistics: A practical guide to data collection and analysis. London: Routledge.

Miller, W. (1971). The death of language or serendipity among the Shoshoni. Anthropological Linguistics, 13(3), 114-120.

Milroy, L. (1980). Language and social networks. In. London: Basil Blackwell.

Milroy, L. (1987). Language and social networks (2nd ed.). Oxford: Wiley-Blackwell.

Mirvahedi, S. H. (2014). Language policy, language practices and language shift in Tabriz. (Unpublished PhD thesis). Victoria University of Wellington: New Zealand,

Montrul, S. (2016). The acquisition of heritage languages. Cambridge: Cambridge University Press.

Morales, K. (2019). "I always knew it ... digo, quizás no perfect": Transnational acts of identity in the speech of a returnee migrant. Trabalhos em Linguística Aplicada, 58(1), 11-32. doi:10.1590/010318138654296464981

Mufwene, S. S. (2010). Globalization, global English, and world English (es): Myths and facts. In N. Coupland (Ed.), The handbook of language and globalization (pp. 31-55). UK: Blackwell Publishing Ltd.

Mühlhäusler, P. (2000). Language planning and language ecology. Current Issues in Language Planning, 1(3), 306-367. doi:10.1080/14664200008668011

Narayan, P. K., \& Smyth, R. (2003). The determinants of immigration from Fiji to New Zealand: An empirical reassessment using the Bounds Testing Approach. International Migration, 41(5), 33-58. doi:10.1111/j.0020-7985.2003.00260.x

Narayan, S. (2015a). Passion for Hindi creates growth avenues. Retrieved from http://www.indiannewslink.co.nz/passion-for-hindi-creates-growth-avenues/

Narayan, S. (2015b). What is in it for Hindi in New Zealand. Paper presented at the World Hindi Conference, Bhopal, India.

Neazor, C. (1991). Language maintenance and shift in the Wellington Polish community. Wellington Working Papers in Linguistics, 36-55.

New Zealand General Election. (2014). Multicultural New Zealand Policy Proposals. Wellington: New Zealand Federation of Multicultural Councils.

Ngaha, A. (2005). Language and identity in the Māori community. In J. Holmes, M. Maclagan, P. Kerswill, \& M. Paviour-Smith (Eds.), Researching language use and language users (pp. 29-48). 
Ngaha, A. (2011). Te Reo, a language for Māori alone? An investigation into the relationship between the Māori language and Māori identity. (Unpublished PhD thesis). The University of Auckland: New Zealand,

Nguyen, T., \& Hamid, M. (2016). Language attitudes, identity and L1 maintenance: A qualitative study of Vietnamese ethnic minority students. System, 61, 87-97.

Nofal, M. (2011). The language situation among the Indians of Yemen: A sociolinguistic study. (Unpublished MA thesis). Middle East University: Jordan,

Norton, B. (2000). Identity and language learning: Gender, ethnicity and educational change. New York: Longman.

Norton, B. (2001). Non-participation, imagined communities, and the language classroom. In M. Breen (Ed.), Learner contributions to language learning: New directions in research (pp. 159-171). Harlow: Pearson Education.

Norton, B. (2010). Language and identity. In N. Hornberger \& S. McKay (Eds.), Sociolinguistics and language education (pp. 349-369). New York: Multilingual Matters.

Norton, B. (2013). Identity and language learning: Extending the conversation (2nd ed.). Bristol: Multilingual Matters.

Norton, B., \& De Costa, P. (2017). Research tasks on identity in language learning and teaching. Language teaching, 51(1), 90-112. doi:10.1017/S0261444817000325

Norton, B., \& McKinney, C. (2011). An identity approach to second language acquisition. In D. Atkinson (Ed.), Alternative approaches to second language acquisition (pp. 85-106). New York: Routledge.

Norton, B., \& Toohey, K. (2001). Changing perspectives on good language learners. TESOL Quarterly, 35(2), 307-322. doi:10.2307/3587650

Norton Peirce, B. (1995). Social identity, investment, and language learning. TESOL Quarterly, 29(1), 9-31. doi:10.2307/3587803

O'Leary, Z. (2017). The essential guide to doing your research project (3rd Ed.). London: SAGE Publications.

O'reilly, C. (2003). When a language is 'just symbolic': Reconsidering the significance of language to the politics of identity. In G. Hogan-Brun \& S. Wolff (Eds.), Minority Languages in Europe: Frameworks, status, prospects (pp. 16-33). London: Palgrave Macmillan.

Ochs, E. (1992). Indexing gender. In A. Durant \& C. Goodwin (Eds.), Rethinking context: Language as an interactive phenomenon (pp. 335-358). New York: Cambridge University Press.

Ochs, E. (1993). Indexing gender. In B. Miller (Ed.), Sex and gender hierarchies (pp. 146-169). New York: Cambridge University Press.

Oliveira, A., Sadler, T., \& Suslak, D. (2007). The linguistic construction of expert identity in professor-student discussions of science. Cultural Studies of Science Education, 2(1), 119-150. doi:10.1007/s11422-006-9039-4

Olphen, H. v. (1988). Religion and language varieties: The case of Hindi-Urdu. In M. A. Jazayery \& W. Winter (Eds.), Languages and cultures: Studies in honor of Edgar C. Polomé (pp. 741-748). Berlin: Mouton de Gruyter.

Omoniyi, T., \& White, G. (Eds.). (2006). The sociolinguistics of identity. London: Continuum. 
Oriyama, K. (2010). Heritage language maintenance and Japanese identity formation: What role can schooling and ethnic community contact play? Heritage Language Journal, 7(2), 237-272.

Oriyama, K. (2016). Community of practice and family language policy: Maintaining heritage Japanese in Sydney-ten years later. International Multilingual Research Journal, 10(4), 289-307. doi:10.1080/19313152.2016.1198977

Paltridge, B., \& Phakiti, A. (2015). Research methods in applied linguistics: A practical resource. London.

Paulston, C. B. (1994). Linguistic minorities in multilingual settings: Implications for language policies. Philadelphia: John Benjamins.

Pauwels, A. (2016). Language maintenance and shift. Cambridge: Cambridge University Press.

Pauwels, A. (2019). Changing perspectives on language maintenance and shift in transnational settings: From settlement to mobility. In G. Hogan-Brun \& B. O'Rourke (Eds.), The Palgrave Handbook of Minority Languages and Communities (pp. 235-256). London: Palgrave Macmillan.

Pavlenko, A. (2001). "In the world of the tradition, I was unimagined": Negotiation of identities in cross-cultural autobiographies. International Journal of Bilingualism, 5(3), 317-344.

Pedraza, P. (2015). Language maintenance among New York Puerto Ricans. In L. ElíasOlivares, E. A. Leone, R. Cisneros, \& J. R. Gutiérrez (Eds.), Spanish language use and public life in the United States (Vol. 35, pp. 59-72). New York: Mouton Publishers.

Peirce, C. S. (1960). Division of signs. In C. Hartshorne, P. Weiss, \& A. Burks (Eds.), Collected Papers of Charles Sanders Peirce (pp. 227-234). Cambridge, MA: Belknap Press of Harvard University Press.

Perera, N. (2015). The maintenance of Sri Lankan languages in Australia: Comparing the experience of the Sinhalese and Tamils in the homeland. Journal of Multilingual and Multicultural Development, 36(3), 297-312. doi:10.1080/01434632.2014.921185

Pérez-Milans, M. (2015). Language and identity in linguistic ethnography. In S. Preece (Ed.), The Routledge handbook of language and identity (pp. 83-97). London: Routledge.

Peterson, E. (2018). Coffee and Danish in Sanpete County, Utah : An exploration of food rituals and language shift. Paper presented at the Workshop on Immigrant Languages in the Americas (WILA 8). Cascadilla Proceedings Project, Somerville, Copenhagen, Denmark.

Piller, I. (2002). Bilingual couples talk: The discursive construction of hybridity. Amsterdam: J. Benjamins.

Pio, E. (2008). Sari: Indian women at work in New Zealand. Wellington, N.Z.: Dunmore Pub.

Plimmer, C. (1994). Language maintenance and shift in the Italian community in Wellington. Wellington Working Papers in Linguistics, 6, 83-105.

Ponzio, A. (2006). Indexicality: Theory. In K. Brown (Ed.), Encyclopedia of language and linguistics (pp. 596-603). Boston: Elsevier. 
Porcel, J. (2006). The paradox of Spanish among Miami Cubans. Journal of Sociolinguistics, 10(1), 93-110. doi:10.1111/j.1360-6441.2006.00319.x

Purkarthofer, J., \& De Korne, H. (2019). Learning language regimes: Children's representations of minority language education. Journal of Sociolinguistics, 120. doi:10.1111/josl.12346

Pyrko, I., Dörfler, V., \& Eden, C. (2017). Thinking together: What makes Communities of Practice work? Human Relations, 70(4), 389-409. doi:10.1177/0018726716661040

Raghunandan, K. (2012). Hyphenated identities: Negotiating'Indianness' and being Indo-Trinidadian. Caribbean Review of Gender Studies, no. 6, ed. Gabrielle Hosein and Lisa Outar, 1-19.

Rampton, B. (1995). Crossing: Language and ethnicity among adolescents. London: Longman.

Rampton, B. (2007). Neo- Hymesian linguistic ethnography in the United Kingdom. Journal of Sociolinguistics, 11(5), 584-607. doi:10.1111/j.14679841.2007.00341.x

Rampton, B., Maybin, J., \& Roberts, M. (2015). Theory and method in linguistic ethnography. In J. Snell, S. Shaw, \& F. Copland (Eds.), Linguistic ethnography: Interdiscplinary explorations (pp. 14-50). Germany: Palgrave.

Rampton, B., Tusting, K., Maybin, J., Barwell, R., Creese, A., \& Lytra, V. (2004). UK linguistic ethnography: A discussion paper. Linguistic Ethnography Forum. Retrieved from

Www.lingethnog.org.uk/documents/papers/ramptonetal2004.pdf

Rannut, U. (2009). Circassian language maintenance in Jordan. Journal of Multilingual and Multicultural Development, 30(4), 297-310. doi:10.1080/01434630902780723

Rasinger, S. M. (2010). Quantitative methods: Concepts, frameworks and issues. In L. Litosseliti (Ed.), Research Methods in Linguistics. (pp. 49-67). London: Continuum.

Raspayeva, A. (2018). Identity construction through positioning in mealtime narratives of Kazakh-speaking village residencts. (Unpublished $\mathrm{PhD}$ thesis). Georgetown University: The USA,

Révész, A. (2012). Coding second language data: Validly and reliably. In A. Mackey \& S. Gass (Eds.), Research methods in second language acquisition: A practical guide (1st ed., pp. 203-221). Chichester: Wiley-Blackwell.

Revis, M. S. (2015). Family language policies of refugees: Ethiopians and Colombians in New Zealand. (Unpublished PhD thesis). Victoria University of Wellington: New Zealand,

Revis, M. S. (2017). A Bourdieusian perspective on child agency in family language policy. International Journal of Bilingual Education and Bilingualism, 19, 1-15. doi:10.1080/13670050.2016.1239691

Riazi, A. M. (2016). The Routledge Encyclopedia of research methods in applied linguistics. Abingdon: Routledge.

Roberts-Holmes, G. (2018). Doing your early years research project: A step by step guide (4th ed.). Los Angeles: Sage Publications Ltd. 
Roberts, M. (1999). Immigrant language maintenance and shift in the Gujarati, Dutch and Samoan communities of Wellington. (Unpublished PhD thesis). Victoria University of Wellington: New Zealand,

Roberts, M. (2005). Immigrants' attitudes to language maintenance in New Zealand. In A. Bell, R. Harlow, \& D. Starks (Eds.), Languages of New Zealand (pp. 248270). Wellington: Victoria University Press.

Robins, R. H., \& Crystal, D. (2019). Language. In Encyclopaedia Britannica.

Romanov, A. (2000). The Russian diaspora in Latvia and Estonia: Predicting language outcomes. Journal of Multilingual and Multicultural Development, 21(1), 5871. doi:10.1080/01434630008666394

Rutherford, J. (Ed.) (1998). Identity: Community, culture, difference. London: Lawrence \& Wishart.

Salahshoor, M. (2017). Maintaining Farsi as a heritage language in the United States: Exploring Persian parents' attitudes, efforts, and challenges. (Unpublished MA thesis). George Mason University: USA,

Salo, N. N. P., \& Dufva, H. (2017). Words and images of multilingualism: A case study of two North Korean refugees. Applied Linguistics Review, $0(0)$. doi:10.1515/applirev-2016-1066

Sasaki, M. (2013). Introspective methods. In A. J. Kunnan (Ed.), The companion to language assessment (Vol. 4, pp. 1340-1357). Malden: Wiley.

Saunders, J. (2007). 'I don't eat meat': Discourse on food among transnational Hindus. Contributions to Indian Sociology, 41(2), 203-233. doi:10.1177/006996670704100203

Schieffelin, B. B., \& Ochs, E. (1986). Language socialization across cultures. Cambridge: Cambridge University Press.

Schilling, N. (2013). Sociolinguistic fieldwork. Cambridge: Cambridge University Press.

Schnurr, S., Marra, M., \& Holmes, J. (2007). Being (im)polite in New Zealand workplaces: Māori and Pākehā leaders. Journal of Pragmatics, 39(4), 712-729. doi:10.1016/j.pragma.2006.11.016

Schwandt, T. (2007). The SAGE dictionary of qualitative inquiry (3rd ed. ed.). Los Angeles: SAGE.

Schwartz, M., \& Gorbatt, N. (2016). 'Why do we know Hebrew and they do not know Arabic?' Children's meta-linguistic talk in bilingual preschool. International Journal of Bilingual Education and Bilingualism, 19(6), 668-688. doi:10.1080/13670050.2016.1181604

Schwartz, M., \& Verschik, A. (Eds.). (2013). Successful family language policy: Parents, children and educators in interaction. London: Springer.

Seals, C. (2013). Multilingual identity development and negotiation amongst heritage language learners: A study of East European-American schoolchildren in the United States. (Unpublished PhD thesis). Georgetown University: The USA,

Seals, C. (2017a). Pasifika heritage language education in New Zealand. In O. Kagan, M. Carreira, \& C. Chik (Eds.), The Routledge handbook of heritage language education: From innovation to program building (pp. 298-312). New York: Routledge. 
Seals, C. (2017b). Positive and negative identity practices in heritage language education. International Journal of Multilingualism, 1-20. doi:10.1080/14790718.2017.1306065

Seals, C. (2019). 'Choosing a mother tongue': Ukrainian sociolinguistic identity politics. UK: Multilingual Matters.

Seals, C., \& Olsen-Reeder, V. (2017). Te Reo Māori, Samoan, and Ukrainian in New Zealand. In C. Seals \& S. Shah (Eds.), Heritage language policies around the world (pp. 221-236). London: Routledge.

Seals, C., \& Peyton, J. K. (2016). Heritage language education: Valuing the languages, literacies, and cultural competencies of immigrant youth. Current Issues in Language Planning, 18(1), 87-101. doi:10.1080/14664208.2016.1168690

Seals, C., \& Shah, S. (Eds.). (2017). Heritage language policies around the world. London: Routledge.

Sercombe, P., Garner, M., \& Raschka, C. (2006). Editors' Introduction to 'Sociolinguistic Research-Who Wins? Research on, with or for Speakers of Minority Languages'. Journal of Multilingual and Multicultural Development, 27(1), 1-3. doi:10.1080/17447140608668534

Shah, S. (2013). Factors affecting proficiency among Gujarati heritage language learners on three continents. (Unpublished $\mathrm{PhD}$ thesis). Georgetown University: The USA,

Shameem, N. (1995). Hamai log ke boli. Language shift in an immigrant community: The Wellington Indo-Fijians. (Unpublished PhD thesis). Victoria University of Wellington: New Zealand,

Shameem, N. (2017). Fiji Hindi in Fiji. In C. Seals \& S. Shah (Eds.), Heritage language policies around the world. London: Routledge.

Shin, S. (2011). Transnational adoptees, community heritage language schools, and identity. Paper presented at the International Symposium on Bilingualism 8, Oslo, Norway.

Shin, S. (2013). Transforming culture and identity: Transnational adoptive families and heritage language learning. Language, Culture and Curriculum, 26(2), 161-178. doi:10.1080/07908318.2013.809095

Shin, S. (2014). Language learning as culture keeping: Family language policies of transnational adoptive parents. International Multilingual Research Journal, 8(3), 189-207.

Shohamy, E. (2006). Language policy: Hidden agendas and new approaches. New York: Routledge.

Shukla, S. (2006). Hindi. In K. Brown, L. Bauer, M. S. Berns, J. E. Miller, \& G. Hirst (Eds.), Encyclopedia of language and linguistics (2nd ed., Vol. 1, pp. 303-305). Amsterdam: Elsevier.

Siegel, J. (1987). Language contact in a plantation environment: A sociolinguistic history of Fiji. Cambridge; New York: Cambridge University Press.

Sierra, S. (2018). Linguistic and ethnic media stereotypes in everyday talk: Humor and identity construction among friends. Journal of Pragmatics. doi:10.1016/j.pragma.2018.09.007 
Silverstein, M. (1976). Shifters, linguistic categories, and cultural description. In k. Basso \& H. Selby (Eds.), Meaning in anthropology (pp. 11-55). Albuquerque, NM: University of New Mexico.

Silverstein, M. (2003). Indexical order and the dialectics of sociolinguistic life. Language and Communication, 23(3), 193-229. doi:10.1016/S02715309(03)00013-2

Singh, R. (2008). Language use between urban Fijians and Indo-Fijians in Suva: Strengthening ties for a common identity. (Unpublished MA thesis). The University of the South Pacific: Fiji,

Skutnabb-Kangas, T. (2000). Linguistic genocide in education or worldwide diversity and human rights? Mahwah, N.J.: L. Erlbaum Associates.

Smith-Christmas, C. (2016). Family language policy: Maintaining an endangered language in the home. London: Palgrave Macmillan.

Smolicz, J. J. (1980). Language as a core value of culture. RELC Journal, 11(1), 1-13. doi:10.1177/003368828001100101

Smolicz, J. J. (1997). In search of a multicultural nation: The case of Australia from an international perspective. In R. Watts \& J. J. Smolicz (Eds.), Cultural democracy and ethnic pluralism: Multicultural and multilingual policies in education (pp. 52-76). Frankfurt: Peter Lang.

Snow, C. E., \& Beals, D. E. (2006). Mealtime talk that supports literacy development. New directions for child and adolescent development, 2006(111), 51-66.

Solano-Campos, A. (2015). Children's national identity in multicultural classrooms in Costa Rica and the United States. Research in Comparative and International Education, 10(1), 71-94.

Soler, J., \& Zabrodskaja, A. (2017). New spaces of new speaker profiles: Exploring language ideologies in transnational multilingual families. Language in Society, 46(4), 547-566.

Spolsky, B. (2004). Language policy. Cambridge: Cambridge University Press.

Spolsky, B. (2005). Māori lost and regained. In A. Bell, R. Harlow, \& D. Starks (Eds.), Languages of New Zealand (pp. 67-85). Wellington: Victoria University Press.

Spolsky, B. (2012). Family language policy - the critical domain. Journal of Multilingual and Multicultural Development, 33(1), 3-11. doi:10.1080/01434632.2011.638072

Sridhar, K. (1985). Bilingualism in South Asia (India): National/regional profiles and verbal repertoires. Annual Review of Applied Linguistics, 6, 169-186.

Sridhar, K. (1992). Meaning, means, and maintenance. In J. Alatis (Ed.), Georgetown University Roundtable on Languages and Linguistics (pp. 56-65). Washington: Georgetown University Press.

Sridhar, K. (2002). The languages of India in New York. In O. García \& J. Fishman (Eds.), The multilingual Apple: Languages in New York City (2nd ed., pp. 259279). New York: De Gruyter Mouton.

Starfield, S. (2015). Ethnographic research. In B. Paltridge \& A. Phakiti (Eds.), Research methods in applied linguistics: A practical resource (pp. 137-152). London.

Starks, D. (2006). The changing roles of language and identity in the New Zealand Niuean community: Findings from the Pasifika languages of Manukau project. International Journal of Bilingual Education and Bilingualism, 9(3), 374-391. 
Starks, D., Taumoefolau, M., Bell, A., \& Davis, K. (2005). Language as a marker of ethnic identity in New Zealand's Pasifika communities. Paper presented at the Proceedings of the 4th International Symposium on Bilingualism.

Statistics New Zealand. (2013). 2013 Census QuickStats about culture and identity. Retrieved from http://www.stats.govt.nz

Stoffel, H.-P. (1981). Observations on the Serbo-Croatian language in New Zealand. New Zealand Slavonic Journal, 1, 53-64.

Suvarierol, S. (2012). Nation-freezing: Images of the nation and the migrant in citizenship packages. Nations and Nationalism, 18(2), 210-229.

Swarbrick, N. (2005). Indians-Indian communities. Te Ara-the Encyclopedia of New Zealand. Accessed 20 September 2016.

Taher, M. (1970). The Asians. In K. Thomsom \& A. Trlin (Eds.), Immigrants in New Zealand (pp. 38-64). Palmerston North: Massey University.

Tajfel, H. (1978). Differentiation between social groups: Studies in the social psychology of intergroup relations. Oxford: Academic Press.

Talmy, S. (2010). Qualitative interviews in applied linguistics: From research instrument to social practice. Annual Review of Applied Linguistics, 30, 128-148.

Talmy, S. (2011). The interview as collaborative achievement: Interaction, identity, and ideology in a speech event. Applied Linguistics, 32(1), 25-42.

Tannen, D. (2007 [1989]). Talking voices: Repetition, dialogue, and imagery in conversational discourse (2nd ed.). Cambridge: Cambridge University Press.

The Royal Society of New Zealand. (2013). Languages in Aotearoa New Zealand. Wellington, NZ: The Royal Society of New Zealand.

Tiwari, K. (1980). The Indian community in New Zealand: A historical survey. In K. Tiwari (Ed.), Indians in New Zealand: Studies in a sub culture (pp. 1-84). Wellington: Price Milburn.

Totoricaguena, G. (2003). The Basques of New York: A cosmopolitan experience. University of Nevada, Reno: Coleccion Urazandi Bilduma. Vitoria-Gasteiz: Servicio Central de Publicaciones del Gobierno Vasco., and Reno Center for Basque Studies.

Tracy, K. (2002). Everyday talk: Building and reflecting identities. New York: The Guilford Press.

Tracy, K., \& Robles, J. (2013). Everyday talk: Building and reflecting identities (2nd ed.). New York: The Guilford Press.

Trentman, E. (2013). Imagined communities and language learning during study abroad: Arabic learners in Egypt. Foreign Language Annals, 46(4), 545-564.

Trudgill, P. (2013). On the fragility of language status: Abstand, distance, and the ecology of Ausbau sociolinguistics. In W. Vandenbussche, E. H. Jahr, \& P. Trudgill (Eds.), Language ecology for the 21 st century: Linguistic conflicts and social environments (pp. 115-134). Oslo: Novus Press.

Trudgill, P., \& Hannah, J. (2002). International English: A guide to the varieties of standard English (4th ed.). London: Arnold.

Tusting, K., \& Maybin, J. (2007). Linguistic ethnography and interdisciplinarity: Opening the discussion. Journal of Sociolinguistics, 11(5), 575-583. 
Tyrrell, N., Guijarro-Fuentes, P., \& Blandon, C. (2014). Intergenerational heritage language practices: A case study of Spanish-speaking families in Britain. Applied Linguistics Review, 5(2), 307-328.

Udah, H. (2018). 'Not by Default Accepted': The African experience of othering and being othered in Australia. Journal of Asian and African Studies, 53(3), 384-400.

Umali, R., \& Bell, A. (2017). Tagalog language maintenance and shift in the New Zealand Filipino community. In M. Marra \& P. Warren (Eds.), Linguist at Work: Festschrift for Janet Holmes (pp. 113-136). Wellington: Victoria University Press.

Umansky, I. M. (2016). To be or not to be EL: An examination of the impact of classifying students as English learners. Educational Evaluation and Policy Analysis, 38(4), 714-737.

UNESCO ad hoc expert group on endangered languages. (2003). Language vitality and endangerment. Retrieved from Paris:

Valdés, G. (2001). Heritage language students: Profiles and possibilities. In J. K. Peyton, D. Ranard, \& S. McGinnis (Eds.), Heritage languages in America: Preserving a national resource (pp. 37-77). Washington D.C.: Center for Applied Linguistics.

Valdés, G. (2014). Heritage language students: Profiles and possibilities. In T. G. Wiley, J. Kreeft Peyton, D. Christian, S. C. K. Moore, \& N. Liu (Eds.), Handbook of heritage, community, and native American languages in the United States: Research, policy, and educational practice (pp. 27-35). London: Routledge.

Vine, B., \& Marra, M. (2017). The Wellington language in the workplace project: Creating stability through flexibility. In M. Marra \& P. Warren (Eds.), Linguist at work: Festschrift for Janet Holmes (pp. 177-197). Wellington: Victoria University Press.

Volkman, T. A. (2005). Introduction: New geographies of kinship. In T. A. Volkman (Ed.), Cultures of transnational adoption (pp. 1-22). Durham, NC: Duke University Press.

Von Krogh, G., Ichijo, K., \& Nonaka, I. (2000). Enabling knowledge creation: How to unlock the mystery of tacit knowledge and release the power of innovation. New York: Oxford University Press.

Waite, J. (1992). Aoteareo: Speaking for ourselves. Wellington: Learning Media, Ministry of Education.

Walker, U. (2011). The role of community in preserving Spanish in New Zealand. In K. Potowski \& J. Rothman (Eds.), Bilingual Youth: Spanish in English-speaking societies (Vol. 42, pp. 331-354). Amsterdam, The Netherlands: John Benjamins Publishing.

Wang, S., \& García, M. (2002). Heritage language learners. National Council of State Supervisors of Foreign Languages: Position Paper. http://www.personal.psu.edu/kej1/blogs/calper_hhl_workshop_su2010/Wang\% 20\%26\%20Garcia\%202002.pdf.

Wanhalla, A. (2013). Matters of the heart : A history of interracial marriage in New Zealand. Auckland: Auckland University Press.

Warren, P. (2016). Uptalk: The phenomenon of rising intonation. Cambridge: Cambridge University Press. 
Weber, J. J. (2014). Flexible multilingual education: Putting children's needs first. Buffalo: Multilingual Matters.

Wei, L. (2020). Ethnography: Origins, features, accountability, and criticality. Abingdon: Routledge.

Weinreich, U. (1974). Languages in contact: Findings and problems. The Hague: Mouton.

Weldeyesus, W. M. (2009). Language socialization and ensuing identity construction among Ethiopian immigrants in metropolitan Denver. (Unpublished $\mathrm{PhD}$ thesis). University of Colorado: The USA,

Wenger, E. (1998a). Communities of practice: Learning as a social system. Systems thinker, 9(5), 1-10.

Wenger, E. (1998b). Communities of practice: Learning, meaning, and identity. Cambridge: Cambridge University Press.

Wenger, E. (2010). Conceptual tools for CoPs as social learning systems: Boundaries, identity, trajectories and participation. In B. Chris (Ed.), Social Learning Systems and Communities of Practice (pp. 125-143). London: Springer.

Wenger, E., McDermott, R. A., \& Snyder, W. (2002). Cultivating communities of practice: A guide to managing knowledge. Boston: Harvard Business School Press.

Williams, G. (2010). Growing up Indian: Problems of cultural identity. In S. Bandyopadhyay (Ed.), India in New Zealand: Local identities, global relations (pp. 107-122). Dunedin: Otago University Press.

Wilson, N. A. (2011). Leadership as communicative practice: The discursive construction of leadership and team identity in a New Zealand rugby team. (Unpublished PhD thesis). Victoria University of Wellington: New Zealand,

Wohlfart, I. (2017). Intergenerational consequences of lifestyle migration: Germanspeaking immigrants in New Zealand. Singapore: Springer.

Wu, M.-H., Lee, K., \& Leung, G. (2014). Heritage language education and investment among Asian American middle schoolers: Insights from a charter school. Language and Education, 28(1), 19-33. doi:10.1080/09500782.2013.763818

Yakushkina, M., \& Olson, D. (2017). Language use and identity in the Cuban community in Russia. Journal of multilingual and multicultural development., 38(1), 50-64. doi:10.1080/01434632.2016.1159686

Yilmaz, B. (2016). Learning "my" language: Moments of languages and identities among Kurds in the UK. (Unpublished PhD thesis). University of London: The UK,

Yoder, M. M., \& Johnstone, B. (2018). Unpacking a political icon: 'Bike lanes' and orders of indexicality. Discourse \& Communication, 12(2), 192-208. doi:10.1177/1750481317745753

Yu, S. (2010). How much does parental language behaviour reflect their language beliefs in language maintenance? Journal of Asian Pacific Communication, 20(1), 1-22. 


\section{Appendix A}

\section{What Is in It for Me? The Benefits of Being Bilingual}

Walking down the strat, you can see many peepie engaged in eanversations with their companions. Someone whose language seerrs comprehensible to you whie another is totally uniknown. While observing this colourful scene in world languages, someone is speaking a lenguage and they, out of theblue, switch to another as they come across a friend, or answer the phone. Around the worid, approximately $70 \%$ of the world population specks at last two languages. Several countries have more then one official national language. For example, South Africa has 11 official languages and New Zealand has two de jure ofticial languages, te reo Máori and New Zealand Sign language (NZSL), and one defacto official language, English.

Pecple are increasingly keen to speak at least one of the 'super-central' languages, such as English, Chinese, Arabic, Hindi, and many others, other than their mother tongues. Being a monolingual means you are in the mincrity of this multaingual world. But. ARE THERE ANY DIFFERENCES BETWEEN BILINGUAL AND MONOLINGUAL PEOPLE? A study conducted at the University of San fiaftaele in Milan (Jubin Abutalebi at al, 2012), reports that bilingual poople use their brains more often than monolinguals and this makes it possible to distinguish bilinguals from manolnguals by looking at scans of their brains. Now, the question is WHAT ARE THE ADVANTAGES OF BEING BILINGUAL? Being bilingual brings about a plethora of benefits. These benefts could be relevant to three categoriss, ie. health \& wellbeng, education and future success.

First, biingualism plays a vital role in the perscns health and wellbeing. Bilingualiem can slow the effects of old age. A Study conducted in Rctman Research Institule at Baycrest in Caneda (Crsik, F..., Bialystok, E. \&amp; Freedman, M. 2010) reports that Ifelong bilingualism confers protection against the onset of Alzheimer disease. it also serves as abridge for connecling people. So being bilingual opens up aocial and cultural oppcrturities, and gives you a new perspective. Such a process of identity and cultural engagement leads to praiseworthy social outcomes that help people enjoy happier ife. Second, education is necessary to us as flight is to bircs. Scientists, scholars, researchers and teachers exert tremendous efforts to ensure thet our children accrue high qualty education. One of the centra benefts of bilingualism is enhancing higher-order thinking skills, e.g. creative thinking, language and multitasking skills. Moreover, bilinguals are able to learn new words easily, use information in new ways, and have good listening akills when compared to monolingual counterparts. Third, future success is one of the tundamental issues that we think of, especially for our children. In addrian to the intellectual skills mastered through formal education, being bilingual can improve ycur competiniveness in the job market. People who know a second language are more likely to have new career opportunities, and leadership skills, e.g. the ability to come up with creative solutions to problems, This increases the possibitity to be professionally successtul and eam more money.

In a nutshell, to be bilingual noeds determination and dedicated hard work, especially if you are trying raise a bilingual child who is immersed in monolingualism. However, these efforts will ensure a healthy life and a prosperous future. making them prolessiconaly 'their own master'.

Mohammed Nofal is a PhD student in Linguistics at Victoria University of Wellington. Before coming to Wellington, ha completed MA in English language and literature majoring in Sociolinguistics (2011) from the Middle East Universiy, Jordan. Before that, he completed BA in English and French (2004) from Aden University, Yamen. He served as a toacher of EFC in the Department al Education, UNIRWA. His tesearch interests are focused on minarity languages, linguistic landscape, and multilingualsm. Mohammed is currently doing his field study in Wellington Hindi School. His PhD research topic is "Language maintenance and shift within the Wellington Hindi communily". 


\section{Appendix B}

\section{Interview guide with community members (Parents):}

1. How many languages do you speak? Name them.

2. What languages do you speak at home? With your children/siblings?

3. If yes, have you ever used Hindi outside of your home? Where? With whom?

4. What language(s) do you use when you meet a new (Indian) person?

5. If you meet a person who speaks English, Hindi, another Indian language (that you speak), what language(s) do you use (1st choice, 2nd and third)?

6. What are the benefits of speaking Hindi?

7. As a parent, how would you feel if your kids spoke only English?

8. In your opinion, what is the future of Hindi in NZ?

9. How do you think your kids identify?

10. How do you identify?

11. Do you send your kids to the Hindi School?

12. What do they think of it? 


\section{Appendix C}

\section{Interview guide with the HS Staff:}

1. Is there an official curriculum to adopt at school? If yes, what is it?

2. If no, what do you use to guide/plan your classes? Who decides?

3. What is the language policy at school? Who set it?

4. What language(s) is used as the medium of instruction?

5. What language(s) do students speak when talking to each other?

6. What support does the school receive from NZ?

7. What support does the school receive from India?

8. What are the benefits of teaching Hindi to the younger generation?

9. Are there any criteria to choose a teacher to teacher a certain level?

10. Why do you teach this level? OR how did you end up teaching this level? Was it your choice?

11. Why do you teach oral first?

12. How would you feel if your kids spoke only English?

13. In your opinion, what is the future of Hindi in NZ?

14. How do you identify?

15. How do you see the students (e.g. Hindi speakers, English speakers, Hindi learners...etc)? 


\section{Appendix D}

Transcription conventions (Following Holmes, Marra, \& Vine, 2011) modified

[ laugh ] Paralinguistic and editorial information in square brackets; colon indicates start and finish

$+\quad$ Untimed pause of approximately one second

//herel Overlapping talk. Double slashes indicate beginning and end

/herell

( ) Untranscribable talk

(word) Transcriber's best guess at an unclear utterance

!? $\quad$ Regular punctuation

WORD stressed word

word Hindi 


\section{Appendix E}

TE WHARE WĀNANGA O TE ŪPOKO O TE IKA A MĀUI

59

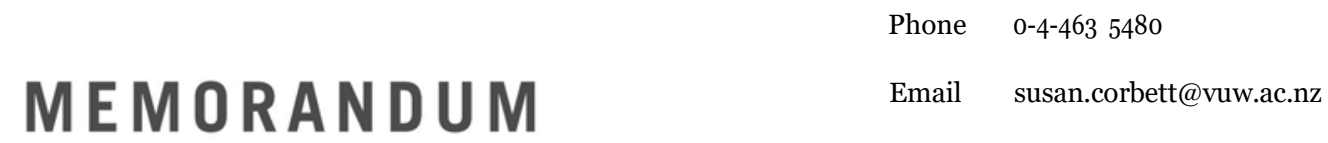

\begin{tabular}{l|l}
\hline TO & Mohammed Nofal \\
\hline COPY TO & Dr Corinne Seals \\
\hline FROM & AProf Susan Corbett, Convener, Human Ethics Committee \\
\hline
\end{tabular}

\begin{tabular}{l|l}
\hline DATE & 28 July 2017 \\
\hline PAGES & 1 \\
\hline
\end{tabular}

SUBJECT Ethics Approval: 24220

Language Maintenance and Shift within the Wellington Hindi Community

Thank you for your request to amend your ethics approval. This has now been considered and the request granted.

Your application has approval until 1 September 2019. If your data collection is not completed by this date you should apply to the Human Ethics Committee for an extension to this approval.

Best wishes with the research. Kind

regards

Susan Corbett

Convener, Victoria University Human Ethics Committee 


\section{Appendix F}

\section{Providing Hindi Education since 1992 \\ www.wellingtonhindischool.org.nz \\ च 38 Priscilla Crescent, Wellington, \& 6449728465}

30 May 2017

To whom it may concern

\section{Mohammed Nofal, PhD Candidate}

I'm pleased to confirm that Mohammed Nofal, PhD candidate from Victoria University of Wellington has the permission of Wellington Hindi School to collect research observations.

Mohammed will be making ten observations from August to December 2017. Six of these observations will be audio recorded.

We are happy to have Mohammed working within our community.

Yours sincerely

Sunita Narayan

Operations Manager

Wellington Hindi School 